\title{
Search for neutral Higgs bosons decaying to tau pairs produced in association with $b$-quarks at $\sqrt{s}=1.96$ $\mathrm{TeV}$
}

\author{
A Dissertation Presented \\ by \\ Kenneth Richard Herner \\ to \\ The Graduate School \\ in Partial Fulfillment of the Requirements \\ for the Degree of \\ Doctor of Philosophy \\ in \\ Physics
}

Stony Brook University

December 2008 


\section{Stony Brook University}

The Graduate School

\section{Kenneth Richard Herner}

We, the dissertation committee for the above candidate for the Doctor of Philosophy degree, hereby recommend acceptance of this dissertation.

John D. Hobbs - Dissertation Advisor

Professor, Department of Physics and Astronomy

Jacobus Verbaarschot - Chairperson of Defense

Professor, Department of Physics and Astronomy

Robert L. MacCarthy

Professor, Department of Physics and Astronomy

Todd Adams

Professor of Physics

Florida State University

This dissertation is accepted by the Graduate School.

Lawrence Martin

Dean of the Graduate School 


\section{Abstract of the Dissertation \\ Search for neutral Higgs bosons decaying to tau pairs produced in association with $b$-quarks at $\sqrt{s}=1.96 \mathrm{TeV}$ \\ by}

Kenneth Richard Herner

Doctor of Philosophy

in

Physics

Stony Brook University

2008

We report results from a search for neutral Higgs bosons decaying to tau pairs produced in association with a b-quark in $1.6 \mathrm{fb}^{-1}$ of data taken from June 2006 to March 2008 with the DØ detector at Fermi National Accelerator Laboratory. The final state includes a muon, hadronically decaying tau, and jet identified as coming from a $b$-quark. We set cross section times branching ratio limits on production of such neutral Higgs bosons $\phi$ in the mass range from $90 \mathrm{GeV}$ to $160 \mathrm{GeV}$. Exclusion limits are set at the $95 \%$ Confidence Level for several supersymmetric scenarios. 


\section{Contents}

List of Figures $\quad$ viii

List of Tables $\quad$ xvii

Acknowledgements $\quad$ xx

1 Introduction 1

1.1 The Fundamental Forces and Particles . . . . . . . . . . . 1

1.2 The Standard Model . . . . . . . . . . . . . . . . . . . . . . 3

1.2 .1 Formalism . . . . . . . . . . . . . . . 3

1.2.2 The Higgs Mechanism _. . . . . . . . . . . . . 5

1.2.3 Challenges to the Standard Model . . . . . . . . . 6

1.3 Supersymmetry . . . . . . . . . . . . . . . . . 7

$1.3 .1 \quad$ MSSM . . . . . . . . . . . . . . . . . . 7

1.3.2 Higgs Phenomenology in the MSSM . . . . . . . . . 8

1.4 Experimental Signatures . . . . . . . . . . . . . . . . . 10

2 Experimental Apparatus $\quad 14$

2.1 Fermi National Accelerator Laboratory . . . . . . . . . . . . . 14

2.1.1 Accelerator Complex . . . . . . . . . . . . . . . . 14

2.1 .2 The Tevatron . . . . . . . . . . . . . . . 17

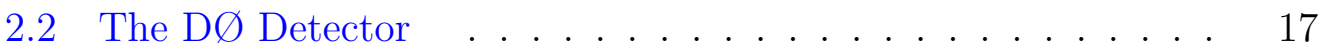

2.2.1 The D $\varnothing$ Coordinate System . . . . . . . . . . . 18

2.2.2 Silicon Microstrip Tracker . . . . . . . . . . . . . 19

2.2 .3 Central Fiber Tracker . . . . . . . . . . . . . . . . 21

2.2 .4 Solenoid and Toroid . . . . . . . . . . . . . . . . . 22

2.2.5 Central and Forward Preshower Detectors . . . . . . 23

2.2 .6 Calorimeter . . . . . . . . . . . . . . . . . . 23

2.2 .7 Muon System . . . . . . . . . . . . . . . . . 25 
3 Trigger and Data Acquisition $\quad 29$

3.1 The D $\varnothing$ Trigger System . . . . . . . . . . . . . . . . . . . . 29

3.1.1 Level 1 Trigger . . . . . . . . . . . . . . . . . . . . . 29

3.1 .2 Level 2 Trigger . . . . . . . . . . . . . . . . . . . 32

3.1 .3 Level 3 Trigger . . . . . . . . . . . . . . . . . . . . 34

3.2 Trigger Design and Implementation . . . . . . . . . . . . . 34

3.2 .1 Single Muon Triggers . . . . . . . . . . . . . . . 34

3.2 .2 Muon + Tau Triggers . . . . . . . . . . . . . . . 36

3.3 Trigger Efficiency Determination . . . . . . . . . . . . . . . 37

3.3.1 Single Muon Triggers . . . . . . . . . . . . . . . . . 37

$3.3 .2 \mathrm{Mu}+$ Tau Triggers . . . . . . . . . . . . . . . . . . . 38

3.3 .3 Trigger ORing . . . . . . . . . . . . . . . . . . . . . . 39

3.3.4 Single Muon OR . . . . . . . . . . . . . . . . . . . 39

$3.3 .5 \mathrm{Mu}+$ Tau OR . . . . . . . . . . . . . . 39

3.3.6 Totally Awesome OR (OR of ORs) . . . . . . . . . 41

3.4 Data Acquisition . . . . . . . . . . . . . . . . 45

4 Event Reconstruction and Object Identification 46

4.1 Event Reconstruction . . . . . . . . . . . . . . . . . 46

4.1 .1 Track Reconstruction . . . . . . . . . . . . . . . . 46

4.1.2 Primary Vertex Reconstruction . . . . . . . . . . . 47

4.1 .3 Muon Reconstruction . . . . . . . . . . . . . . . . . . 48

4.1 .4 Jet Reconstruction . . . . . . . . . . . . . . . . . . 49

4.1.5 Tau Reconstruction . . . . . . . . . . . . 50

4.2 Object Identification . . . . . . . . . . . . . . . . . . 53

4.2 .1 Tau-ID . . . . . . . . . . . . . . . . . . . . 53

4.2 .2 Muon-ID . . . . . . . . . . . . . . . . . . . . . 55

$4.2 .3 \quad b$-ID $\ldots \ldots \ldots \ldots \ldots \ldots \ldots \ldots$

5 Simulation $\quad \mathbf{6 2}$

5.1 Event Generators . . . . . . . . . . . . . . . . . . . 62

5.2 Detector simulation and reconstruction . . . . . . . . . 63

5.2 .1 dogstar . . . . . . . . . . . . . . . . . . 63

$5.2 .2 \mathrm{~d} 0 \operatorname{sim} \ldots \ldots \ldots \ldots \ldots \ldots$

5.2 .3 Reconstruction . . . . . . . . . . . . . . 63

5.3 Simulation corrections . . . . . . . . . . . . . . . 63

5.3 .1 Muon ID Corrections . . . . . . . . . . . . . . 63

5.3 .2 Tau-ID corrections . . . . . . . . . . . . . . . 64

5.3 .3 Jet Corrections . . . . . . . . . . . . . . . . . . 65

5.3.4 Luminosity Reweighting . . . . . . . . . . . . . . 65

5.3.5 Beam position Reweighting . . . . . . . . . 66 
$5.3 .6 \quad Z-p_{T}$ Reweighting $\ldots \ldots \ldots \ldots \ldots$

$6 \quad \phi b$ Analysis Methodology $\quad 67$

6.1 Dataset and Monte Carlo Samples . . . . . . . . . . . . . . 67

6.1 .1 Data . . . . . . . . . . . . . . . 67

6.1 .2 Monte Carlo . . . . . . . . . . . . . . . . 67

6.2 Event Preselection . . . . . . . . . . . . . . . . . . . . 71

6.2 .1 Jet Selection . . . . . . . . . . . . . . . . . 72

6.3 QCD Multijet Background Estimation . . . . . . . . . 76

6.3.1 QCD estimation in pre-btag sample . . . . . . . . . 77

6.3.2 QCD estimation after $b$-tagging $\ldots \ldots \ldots \ldots \ldots$

6.3.3 Discussion and Final QCD results . . . . . . . . . . 82

6.4 Multivariate Techniques . . . . . . . . . . . . . . . 83

6.4.1 Top Identification Neural Network (KNN) . . . . . . . 83

6.4 .2 QCD Likelihood . . . . . . . . . . . . . . . . 84

6.4.3 Final Discriminant Variable . . . . . . . . . . . . . 90

6.5 Systematic Uncertainties . . . . . . . . . . . . . . . . . . . 91

$\begin{array}{lll}7 & \text { Results } & 98\end{array}$

7.1 Cross Section Limit _ . . . . . . . . . . . . . . . . . 98

7.2 Interpretation within the MSSM . . . . . . . . . . . 101

8 Conclusions 104

8.1 Conclusions . . . . . . . . . . . . . . . . . . . . . . 104

8.2 Outlook . . . . . . . . . . . . . . . . . . . . 104

$\begin{array}{ll}\text { Bibliography } & 106\end{array}$

Appendix A The Silicon Track Trigger 111

A.1 Introduction . . . . . . . . . . . . . . . . . . . . . 111

A.2 Technical Design . . . . . . . . . . . . . . . . . . 112

A.2.1 Fiber Road Card . . . . . . . . . . . . . . . . . . 114

A.2.2 Silicon Trigger Card . . . . . . . . . . . . 115

A.2.3 Track Fit Card . . . . . . . . . . . . . . . . 116

A.3 Performance . . . . . . . . . . . . . . . . . . . 121

A.3.1 Performance in $Z \rightarrow \mu \mu$ events $\ldots \ldots \ldots \ldots . \quad 122$

A.3.2 Performance in multijet events . . . . . . . . . . . 123

A.3.3 Use in trigger design . . . . . . . . . . . . . . 125

Appendix B Data/Monte Carlo Comparison Plots 126

B.1 Data/Monte Carlo Plots Before $b$-tagging . . . . . . . . . . 127

B.2 Data/Monte Carlo Plots After $b$-tagging . . . . . . . . 146 


\section{List of Figures}

1.1 Relative coupling constants of the three gauge symmetries as a function of energy in the Standard Model (left) and the MSSM (right). We see that the couplings converge to a common point in the MSSM [11]. . . . . . . . . . . . . . . . . .

1.2 Tree-level diagrams of $h b$ production. There are similar diagrams for $A b$ and $H b$ production. . . . . . . . . . . . . . . . . 10

1.3 Standard model $\phi b$ production cross section in $\mathrm{fb}$ at the Tevatron. The associated $b$-quark has transverse momentum, or $p_{T}$, grater than $15 \mathrm{GeV}$ and $|\eta|<2.0[13] . \quad \eta=-\ln \left(\tan \frac{\theta}{2}\right)$ (Sec.2.2.1). In the MSSM, the production cross sections can be significantly enhanced over these shown here by a factor of order $\tan ^{2} \beta \ldots \ldots \ldots \ldots \ldots \ldots$

1.4 Branching ratios of the scalar MSSM Higgs bosons $h$ and $H$ at

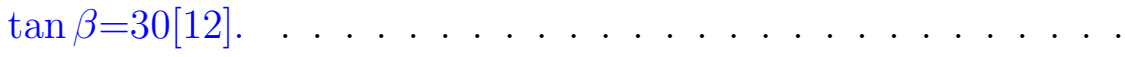

1.5 Branching ratios of the pseudoscalar MSSM Higgs boson $A$ at $\tan \beta=30[12] \ldots \ldots \ldots \ldots \ldots$

2.1 Overview of Fermilab, the Tevatron, and the associated accelerators. The Tevatron has a $1 \mathrm{~km}$ radius; everything is drawn to the appropriate scale. [20]. . . . . . . . . . . . .

2.2 Side view of the $\mathrm{D} \varnothing$ detector with major subsystems labeled $[22] \ldots \ldots \ldots \ldots \ldots \ldots \ldots$

2.3 SMT layout [22]. The two outer H-disks were removed during the 2006 RunIIb upgrade. . . . . . . . . . . . . .

2.4 SMT disk/barrel module cross section [22]. Layer 0, added in 2006, is not shown. . . . . . . . . . . . . .

2.5 Side view of the $\mathrm{D} \varnothing$ central tracker showing the SMT and CFT[22].

2.6 Geometry of the preshower scintillator strips. The circles show the of the wavelength-shifting fibers (WLS) [22]. . . . . . . 24

2.7 The $\mathrm{D} \varnothing$ calorimeter[22]. . . . . . . . . . . . . . . . . 25 
2.8 Cross section of the $\mathrm{D} \varnothing$ calorimeter showing tower and cell segmentation. The shading patterns show cells that are grouped together into a tower during signal readout[22]. . . . . . . . 26

2.9 Design of a typical D $\varnothing$ calorimeter cell[22] . . . . . . . . . . 26

2.10 An exploded view of the muon system, the outermost part of the $\mathrm{D} \varnothing$ detector. Only the wire, i.e. drift chamber, portion is shown here.[22]. . . . . . . . . . . . . . . .

2.11 An exploded view of the muon system, with the scintillator systems ( $A \phi$ scintillators and cosmic caps in the central system, trigger scintillation counters in the forward system) shown [22].

3.1 Overview of the $\mathrm{D} \varnothing$ trigger system [22]. . . . . . . . . . . . . 29

3.2 Diagram of the Level 1 and Level 2 trigger system [22]. . . . . 30

3.3 1-D efficiency projections of the OR of the single muon triggers. Upper right: efficiency vs. detector $\eta$. Lower left: efficiency vs. $\phi$. Lower right: efficiency vs. $p_{T}$. The efficiency has been measured with respect to the same reconstructed muon quality cuts (loose muon, medium track match) as we make in the analysis.

3.4 1-D efficiency projections of the OR of muon terms from the MTA triggers. Upper right: efficiency vs. detector $\eta$. Lower left: efficiency vs. $\phi$. Lower right: efficiency vs. $p_{T}$. The efficiency has been measured with respect to the same muon quality cuts (loose muon, medium track match) as we make in the analysis. . . . . . . . . . . . . . . .

3.5 1-D efficiency projections of firing at least one single muon trigger and at least one muon term from a MTA trigger. Upper right: efficiency vs. detector $\eta$. Lower left: efficiency vs. $\phi$. Lower right: efficiency vs. $p_{T}$. The efficiency has been measured with respect to the same muon quality cuts (loose muon, medium track match) as we make in the analysis. . . . . . . .

3.6 Efficiency of the OR of tau trigger terms parametrized in absolute value of detector $\eta$ (Y-axis) and $E_{T}$ (X-axis). . . . . .

3.7 Efficiency vs. tau $E_{T}$ for type 2 taus (Sec. 4.1.5.) Red is the OR of the tau object conditions, Blue is the MTA3_TLM10T10NN1 trigger only, and black is the MTA1_TLM10T10NN1 trigger only. One can easily see that the two individual triggers are complimentary and the resulting efficiency improves over the entire range. . . . . . . . . . . . . . . . . . . 44

3.8 Dataflow through the DØ DAQ system [22]. . . . . . . . . 45 
4.1 Offset correction to the jet energy scale for a jet cone size of 0.5 as a function of jet $\eta$ as measured from the center of the detector. The different curves represent different primary vertex multiplicities[41]. . . . . . . . . . . . . . .

4.2 Relative response correction to the jet energy scale in a photon+jet sample (left) and di-jet sample (right) [41]. . . . . . .

4.3 Absolute response correction to the jet energy scale for a jet cone size of 0.5 [41]. . . . . . . . . . . . . . . .

4.4 Jet showering correction to the jet energy scale as a function of jet energy for a jet cone size of $0.5[41]$. . . . . . . . . . . .

4.5 $N N_{\tau}$ Output for type 1 taus in data (upper left), data with muon-tau overlaps (upper right), $W \rightarrow \mu \nu \mathrm{MC}$ events (lower left), and $Z \rightarrow \tau \tau \mathrm{MC}$ events (lower right)[43]. . . . . . . . .

4.6 $N N_{\tau}$ Output for type 2 taus in data (upper left), data with muon-tau overlaps (upper right), $W \rightarrow \mu \nu \mathrm{MC}$ events (lower left), and $Z \rightarrow \tau \tau \mathrm{MC}$ events (lower right)[43]. . . . . . . . .

4.7 $N N_{\tau}$ Output for type 3 taus in data (upper left), data with muon-tau overlaps (upper right), $W \rightarrow \mu \nu \mathrm{MC}$ events (lower left), and $Z \rightarrow \tau \tau \mathrm{MC}$ events (lower right)[43]. . . . . . . . .

4.8 Efficiency of the NN $b$-tagger for the TIGHT operating point in $\mathrm{MC}\left(\right.$ red) and data(green) as a function of jet $p_{T}$ in the $\mid \eta<1$, or $\mathrm{CC}$, region (upper left); the $1<\mid \eta<1.8$, or Inter-Cryostat Region (ICR) (upper right); the $1.8<\mid \eta<2.5$, or EC, region (lower left); and as a function of $|\eta|$ (bottom right) [46]. . . .

4.9 Fake tag rate of the NN $b$-tagger for the TIGHT operating point in $\mathrm{MC}$ (red) and data(green) as a function of jet $p_{T}$ in the CC(red), ICR(blue), and EC(green). The dashed lines represent the uncertainties $[46] . \ldots \ldots \ldots . \ldots$

6.1 Tau $p_{T}$ before $b$-tagging for: Type 1 taus (upper left), Type 2 (upper right), Type 3 (lower left), and all types (lower right) using the Single Muon or Mu+Tau OR. . . . . . . . . . . . .

6.2 Muon $p_{T}$ before $b$-tagging for: Type 1 taus (upper left), Type 2 (upper right), Type 3 (lower left), and all types (lower right) using the Single Muon or Mu+Tau OR. . . . . . . . . . . . . .

6.3 Invariant mass of the $\mu, \tau, \mathbb{E}_{T}$ system before $b$-tagging for: Type
1 taus (upper left), Type 2 (upper right), Type 3 (lower left),
and all types (lower right). . . . . . . . . . . . . . .
6.4 Tau $p_{T}$ after $b$-tagging for: Type 1 taus (upper left), Type 2

6.3 Invariant mass of the $\mu, \tau, \mathbb{E}_{T}$ system before $b$-tagging for: Type
1 taus (upper left), Type 2 (upper right), Type 3 (lower left),
and all types (lower right). . . . . . . . . . . . . . . . . Type 1 taus (upper left), Type 2

6.3 Invariant mass of the $\mu, \tau, \mathbb{E}_{T}$ system before $b$-tagging for: Type
1 taus (upper left), Type 2 (upper right), Type 3 (lower left),
and all types (lower right). . . . . . . . . . . . . . . . . Type 1 taus (upper left), Type 2 (upper right), Type 3 (lower left), and all types (lower right) using the Single Muon or Mu+Tau OR. . . . . . . . . . . . 
6.5 Muon $p_{T}$ after $b$-tagging for: Type 1 taus (upper left), Type 2 (upper right), Type 3 (lower left), and all types (lower right) using the Single Muon or Mu+Tau OR. . . . . . . . . . . .

6.6 Invariant mass of the $\mu, \tau, \mathbb{E}_{T}$ system after $b$-tagging for: Type 1 taus (upper left), Type 2 (upper right), Type 3 (lower left), and all types (lower right). . . . . . . . . . . . .

$6.7 b$-tagging rate as a function of jet $p_{T}$, the pseudo TRF, for all tau types (left) and overlaid over data from the separate tau types(right). Green is Type 1, red is Type 2, and blue is Type 3. 80

6.8 Muon isolation rate as a function of muon $p_{T}$ in noiso sample

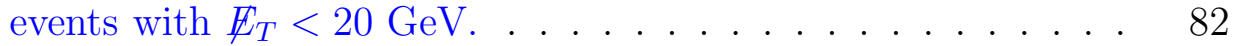

6.9 KNN Input variables before $b$-tagging for all tau types combined: HT (upper left), Energy from 4 vector sum (upper right), Number of reconstructed jets (lower left), $\Delta \phi(\mu, \tau)$ (lower right). 84

$6.10 \mathrm{KNN}$ Input variables after $b$-tagging for all tau types combined: HT (upper left), Energy from 4 vector sum (upper right), Number of reconstructed jets (lower left), $\Delta \phi(\mu, \tau)$ (lower right).

$6.11 \mathrm{KNN}$ output before $b$-tagging for: Type 1 taus (upper left), Type 2 (upper right), Type 3 (lower left), and all types (lower right). . . . . . . . . . . . . . . .

6.12 KNN output after $b$-tagging for: Type 1 taus (upper left), Type 2 (upper right), Type 3 (lower left), and all types (lower right).

6.13 QCD likelihood $(\log R)$ output before $b$-tagging for: Type 1 taus (upper left), Type 2 (upper right), Type 3 (lower left), and all types (lower right). . . . . . . . . . . . . .

6.14 QCD likelihood $(\log R)$ output after $b$-tagging for: Type 1 taus (upper left), Type 2 (upper right), Type 3 (lower left), and all types (lower right). . . . . . . . . . . . . .

6.15 KNN output vs. QCD likelihood output with $120 \mathrm{GeV}$ signal for after $b$-tagging for: Type 1 taus (upper left), Type 2 (upper right), Type 3 (bottom.) . . . . . . . . . . . .

6.16 Final discriminant variable, with $120 \mathrm{GeV}$ signal overlay and using $120 \mathrm{GeV}$ QCD likelihood, before $b$-tagging, for: Type 1 taus (upper left), Type 2 (upper right), Type 3 (lower left), and all types (lower right). . . . . . . . . . . . . .

6.17 Final discriminant variable, with $120 \mathrm{GeV}$ signal overlay and using $120 \mathrm{GeV}$ QCD likelihood, after $b$-tagging, for: Type 1 taus (upper left), Type 2 (upper right), Type 3 (lower left), and all types (lower right). . . . . . . . . . . . . 
6.18 Final discriminant variable, with $120 \mathrm{GeV}$ signal overlay and using $120 \mathrm{GeV}$ QCD likelihood, after $b$-tagging and cuts in Table 6.9, for: Type 1 taus (upper left), Type 2 (upper right), Type 3 (lower left), and all types (lower right). . . . . . . .

7.1 LLR distributions used in the limit setting for each mass point. Row 1: $90 \mathrm{GeV}$ (left), $100 \mathrm{GeV}$ (right). Row 2: $110 \mathrm{GeV}$ (left), $120 \mathrm{GeV}$ (right). Row 3: $130 \mathrm{GeV}$ (left), $140 \mathrm{GeV}$ (right). Row 4: $150 \mathrm{GeV}$ (left), $160 \mathrm{GeV}$ (right). . . . . . . . . . . .

$7.21-C L_{s}$ vs. Higgs mass for the nominal input cross section. The limit setting program slowly increases the signal cross section until this value is 0.05 at that mass point. . . . . . . . . . 101

7.3 Expected and observed cross section $\times$ BR limits vs. Higgs mass. 102

7.4 Limits on $\tan \beta$ vs. $M_{A}$ for the no-mixing, $\mu>0$ case (upper left); no-mixing, $\mu<0$ case (upper right); maximal-mixing, $\mu>0$ case (lower left); maximal-mixing, $\mu<0$ case (lower right).103

A.1 STT pattern recognition. Clusters from SMT hits are associated to tracks from L1CTT; the STT then fits the SMT hits to a track by minimizing a $\chi^{2}$ function in $b, \kappa$, and $\phi$. [30]. . . . . 112

A.2 Dataflow through the STT. The Level 3 data paths have been

A.3 Block diagram of the Fiber Road Card (FRC). The dashed rectangle denotes the on-board FRC components [30]. . . . . . . 114

A.4 Block diagram of the Silicon Trigger Card (STC)[30]. . . . . . 115

A.5 Block diagram of the Silicon Trigger Card (STC)[30]. . . . . . 118

A.6 Block diagram of the Track Fit Card (TFC) [30]. . . . . . . . . 119

A.7 Efficiency for a good STT track to be matched to a good RECO

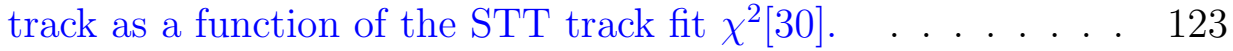

A.8 Efficiency for a good STT track to be matched to a good RECO track as a function of the STT track fit. The different point represent different values of the STT track $\chi^{2}$. Looser cuts have low purity and high efficiency, while tighter cuts have lower efficiency but higher purity. . . . . . . . . . . . .

B.1 Expanded view of the legend used for kinematic and topological variable plots in Appendix B and Chapter 6. Here "HF" means heavy flavor, $b \bar{b}$ or $c \bar{c}$ pairs, and "j" means additional light quark or gluon jets. . . . . . . . . . . . . . . . . .

B.2 $\Delta \phi\left(\mu, \mathbb{E}_{T}\right)$ before $b$-tagging for: Type 1 taus (upper left), Type 2 (upper right), Type 3 (lower left), and all types (lower right). 
B.3 $\Delta \phi\left(\tau, E_{T}\right)$ before $b$-tagging for: Type 1 taus (upper left), Type 2 (upper right), Type 3 (lower left), and all types (lower right).

B.4 $\Delta \phi(\mu, \tau)$ before $b$-tagging for: Type 1 taus (upper left), Type 2 (upper right), Type 3 (lower left), and all types (lower right). .

B.5 The Enu (or $E_{\nu}$ ) variable, $E_{\nu} \equiv \mathbb{E}_{T} E_{\mu} / p_{T \mu}$ before $b$-tagging for: Type 1 taus (upper left), Type 2 (upper right), Type 3 (lower left), and all types (lower right). . . . . . . . . .

B.6 Sum of trasverse energy of all jets $\left(H_{T}\right)$ before $b$-tagging for: Type 1 taus (upper left), Type 2 (upper right), Type 3 (lower left), and all types (lower right). . . . . . . . . . .

B.7 Leading (highest- $E_{T}$ ) jet $p_{T}$ before $b$-tagging for: Type 1 taus (upper left), Type 2 (upper right), Type 3 (lower left), and all types (lower right). . . . . . . . . . . . . .

B.8 Leading (highest- $E_{T}$ ) jet $\eta$ before $b$-tagging for: Type 1 taus (upper left), Type 2 (upper right), Type 3 (lower left), and all types (lower right). . . . . . . . . . . . . . .

B.9 Leading (highest- $E_{T}$ ) jet $\phi$ before $b$-tagging for: Type 1 taus (upper left), Type 2 (upper right), Type 3 (lower left), and all types (lower right). . . . . . . . . . . . . .

B.10 Missing transverse energy $\left(E_{T}\right)$ before $b$-tagging for: Type 1 taus (upper left), Type 2 (upper right), Type 3 (lower left), and all types (lower right).

133

B.11 Energy from 4 -vector sum of muon, tau, and $\mathbb{E}_{T}\left(E_{4}\right)$ before $b$ tagging for: Type 1 taus (upper left), Type 2 (upper right), Type 3 (lower left), and all types (lower right). . . . . . . . .

B.12 $\Delta R(\mu, \tau)$, where $\Delta R=\sqrt{(\Delta \eta)^{2}+(\Delta \phi)^{2}}$, before $b$-tagging for: Type 1 taus (upper left), Type 2 (upper right), Type 3 (lower left), and all types (lower right). . . . . . . . .

B.13 The $\mu, \tau$ invariant mass before $b$-tagging for: Type 1 taus (upper left), Type 2 (upper right), Type 3 (lower left), and all types (lower right).

B.14 Muon $\eta$ before $b$-tagging for: Type 1 taus (upper left), Type 2 (upper right), Type 3 (lower left), and all types (lower right).

B.15 Muon $\phi$ before $b$-tagging for: Type 1 taus (upper left), Type 2 (upper right), Type 3 (lower left), and all types (lower right). The pronounced dip around $\phi=5$ is due to the lack of full instrumentation at the bottom of the detector. . . . . . .

B.16 Jet multiplicity before $b$-tagging for: Type 1 taus (upper left), Type 2 (upper right), Type 3 (lower left), and all types (lower right). . . . . . . . . . . . . . . 
B.17 Tau associated track $p_{T}$ before $b$-tagging for: Type 1 taus (upper left), Type 2 (upper right), Type 3 (lower left), and all types (lower right). In tau type 3 , it is the $p_{T}$ sum of all tracks. . .

B.18 The transverse mass $\left(M_{T}=\sqrt{\left(2 \mathbb{E}_{T} E_{T \mu}\left(1-\cos \left(\Delta \phi\left(\mu, \mathbb{E}_{T}\right)\right)\right)\right.}\right)$ before $b$-tagging for: Type 1 taus (upper left), Type 2 (upper right), Type 3 (lower left), and all types (lower right). . . . . . 143

B.19 The $W$ mass variable $\left(M_{W}=\sqrt{\left(2 E_{T} E_{\mu}^{2} / p_{T \mu}\left(1-\cos \left(\Delta \phi\left(\mu, E_{T}\right)\right)\right)\right)}\right)$ before $b$-tagging for: Type 1 taus (upper left), Type 2 (upper right), Type 3 (lower left), and all types (lower right). . . . . .

B.20 The $Z p_{T}$ variable, $Z p_{T}=\left|\boldsymbol{p}_{\boldsymbol{T}}^{\boldsymbol{\mu}}+\boldsymbol{p}_{\boldsymbol{T}}^{\boldsymbol{\tau}}+\boldsymbol{E}_{\boldsymbol{T}}\right|$, before $b$-tagging for: Type 1 taus (upper left), Type 2 (upper right), Type 3 (lower left), and all types (lower right) using the Single Muon or $\mathrm{Mu}+\mathrm{Tau}$ OR. . . . . . . . . . . . . . . . . . . . . 145

B.21 $\Delta \phi\left(\mu, E_{T}\right)$ after $b$-tagging for: Type 1 taus (upper left), Type 2 (upper right), Type 3 (lower left), and all types (lower right). $\Delta \phi\left(\tau, \mathbb{E}_{T}\right)$ after $b$-tagging for: Type 1 taus (upper left), Type 2 (upper right), Type 3 (lower left), and all types (lower right). .

B.23 $\Delta \phi(\mu, \tau)$ after $b$-tagging for: Type 1 taus (upper left), Type 2 (upper right), Type 3 (lower left), and all types (lower right). . 148

B.24 The Enu (or $E_{\nu}$ ) variable, $E_{\nu} \equiv E_{T} E_{\mu} / p_{T \mu}$ after $b$-tagging for: Type 1 taus (upper left), Type 2 (upper right), Type 3 (lower left), and all types (lower right). . . . . . . . . . . .

B.25 Sum of trasverse energy of all jets $\left(H_{T}\right)$ after $b$-tagging for: Type 1 taus (upper left), Type 2 (upper right), Type 3 (lower left), and all types (lower right). . . . . . . . . . . . . .

B.26 Leading (highest- $E_{T}$ ) jet $p_{T}$ after $b$-tagging for: Type 1 taus (upper left), Type 2 (upper right), Type 3 (lower left), and all types (lower right). . . . . . . . . . . . . .

B.27 Leading (highest- $E_{T}$ ) jet $\eta$ after $b$-tagging for: Type 1 taus (upper left), Type 2 (upper right), Type 3 (lower left), and all types (lower right). . . . . . . . . . . . . .

B.28 Leading (highest- $E_{T}$ ) jet $\phi$ after $b$-tagging for: Type 1 taus (upper left), Type 2 (upper right), Type 3 (lower left), and all types (lower right).

B.29 Missing transverse energy $\left(\mathbb{E}_{T}\right)$ after $b$-tagging for: Type 1 taus (upper left), Type 2 (upper right), Type 3 (lower left), and all types (lower right). 
B.30 Energy from 4-vector sum of muon, tau, and $E_{T}\left(E_{4}\right)$ after $b$ tagging for: Type 1 taus (upper left), Type 2 (upper right), Type 3 (lower left), and all types (lower right). . . . . . . .

B.31 $\Delta R(\mu, \tau)$, where $\Delta R=\sqrt{(\Delta \eta)^{2}+(\Delta \phi)^{2}}$, after $b$-tagging for: Type 1 taus (upper left), Type 2 (upper right), Type 3 (lower left), and all types (lower right). . . . . . . . . . .

B.32 The $\mu, \tau$ invariant mass after $b$-tagging for: Type 1 taus (upper left), Type 2 (upper right), Type 3 (lower left), and all types (lower right).

B.33 Muon $\eta$ after $b$-tagging for: Type 1 taus (upper left), Type 2 (upper right), Type 3 (lower left), and all types (lower right).

B.34 Muon $\phi$ after $b$-tagging for: Type 1 taus (upper left), Type 2 (upper right), Type 3 (lower left), and all types (lower right). The pronounced dip around $\phi=5$ is due to the lack of full instrumentation at the bottom of the detector. . . . . . . .

B.35 Jet multiplicity after $b$-tagging for: Type 1 taus (upper left), Type 2 (upper right), Type 3 (lower left), and all types (lower right). . . . . . . . . . . . . . .

B.36 Tau associated track $p_{T}$ after $b$-tagging for: Type 1 taus (upper left), Type 2 (upper right), Type 3 (lower left), and all types (lower right). In tau type 3 , it is the $p_{T}$ sum of all tracks. . .

B.37 The transverse mass $\left(M_{T}=\sqrt{\left(2 \mathbb{E}_{T} E_{T \mu}\left(1-\cos \left(\Delta \phi\left(\mu, \mathbb{E}_{T}\right)\right)\right)\right.}\right)$ after $b$-tagging for: Type 1 taus (upper left), Type 2 (upper right), Type 3 (lower left), and all types (lower right). . . . . . 162

B.38 The $W$ mass variable $\left(M_{W}=\sqrt{\left(2 \mathscr{E}_{T} E_{\mu}^{2} / p_{T \mu}\left(1-\cos \left(\Delta \phi\left(\mu, \mathbb{E}_{T}\right)\right)\right)\right)}\right)$ after $b$-tagging for: Type 1 taus (upper left), Type 2 (upper right), Type 3 (lower left), and all types (lower right). . . . . 163

B.39 The $Z p_{T}$ variable, $Z p_{T}=\left|\boldsymbol{p}_{\boldsymbol{T}}^{\boldsymbol{\mu}}+\boldsymbol{p}_{\boldsymbol{T}}^{\boldsymbol{\tau}}+\boldsymbol{E}_{\boldsymbol{T}}\right|$, after $b$-tagging for: Type 1 taus (upper left), Type 2 (upper right), Type 3 (lower left), and all types (lower right) using the Single Muon or $\mathrm{Mu}+\mathrm{Tau}$ OR. . . . . . . . . . . . . . . . .

C.1 Muon $p_{T}$ smoothing functions on the QCD background for the QCD likelihood. . . . . . . . . . . . 166

C.2 Tau $p_{T}$ smoothing functions on the QCD background for the QCD likelihood. . . . . . . . . . . . . 166

C.3 $\Delta R(\mu, \tau)$ smoothing functions on the QCD background for the QCD likelihood. . . . . . . . . . . . . 166

C.4 $\mu, \tau$ invariant mass smoothing functions on the QCD background for the QCD likelihood. . . . . . . . . . . 167 
C.5 $\mu, \tau \mathbb{E}_{T}$ invariant mass smoothing functions on the QCD background for the QCD likelihood. . . . . . . . . . . . . 167

C.6 Muon $p_{T}$ smoothing functions for signal likelihood for the different Higgs masses. Row 1: $90 \mathrm{GeV}$ (left), $100 \mathrm{GeV}$ (right). Row 2: $110 \mathrm{GeV}$ (left), $120 \mathrm{GeV}$ (right). Row 3: $130 \mathrm{GeV}$ (left), $140 \mathrm{GeV}$ (right). Row 4: $150 \mathrm{GeV}$ (left), $160 \mathrm{GeV}$ (right). . . 168

C.7 Tau $p_{T}$ smoothing functions for signal likelihood for the different Higgs masses. Row 1: $90 \mathrm{GeV}$ (left), $100 \mathrm{GeV}$ (right). Row 2: $110 \mathrm{GeV}$ (left), $120 \mathrm{GeV}$ (right). Row 3: $130 \mathrm{GeV}$ (left), 140 $\mathrm{GeV}$ (right). Row 4: $150 \mathrm{GeV}$ (left), $160 \mathrm{GeV}$ (right). . . . . 169

C.8 $\Delta R(\mu, \tau)$ smoothing functions for signal likelihood for the different Higgs masses. Row 1: $90 \mathrm{GeV}$ (left), $100 \mathrm{GeV}$ (right). Row 2: $110 \mathrm{GeV}$ (left), $120 \mathrm{GeV}$ (right). Row 3: $130 \mathrm{GeV}$ (left), $140 \mathrm{GeV}$ (right). Row 4: $150 \mathrm{GeV}$ (left), $160 \mathrm{GeV}$ (right). . . 170

C.9 $\mu, \tau$ invariant mass smoothing functions for signal likelihood for the different Higgs masses. Row 1: $90 \mathrm{GeV}$ (left), $100 \mathrm{GeV}$ (right). Row 2: $110 \mathrm{GeV}$ (left), $120 \mathrm{GeV}$ (right). Row 3: 130 $\mathrm{GeV}$ (left), $140 \mathrm{GeV}$ (right). Row 4: $150 \mathrm{GeV}$ (left), $160 \mathrm{GeV}$ (right). . . . . . . . . . . . . . . . . . 171

C.10 $\left(\mu, \tau, \mathbb{E}_{T}\right)$ invariant mass smoothing functions for signal likelihood for the different Higgs masses. Row 1: $90 \mathrm{GeV}$ (left), 100 $\mathrm{GeV}$ (right). Row 2: $110 \mathrm{GeV}$ (left), $120 \mathrm{GeV}$ (right). Row 3: $130 \mathrm{GeV}$ (left), $140 \mathrm{GeV}$ (right). Row 4: $150 \mathrm{GeV}$ (left), 160 $\mathrm{GeV}$ (right). . . . . . . . . . . . . . . . 172 


\section{List of Tables}

1.1 List of the elementary particles and force carriers, including their electromagnetic charge $q$, masses in $\mathrm{GeV}$, and what forces they feel in the case of fermions, or mediate in the case of bosons. $\mathrm{S}$ is the strong force, $\mathrm{E}$ is the electromagnetic part of the electroweak force, and $\mathrm{W}$ is the weak part of the electroweak force. The fermions are also grouped in their three generations, or families. Masses taken from [1]. Gravity is neglected here. . .

1.2 Tau branching ratios [1]. $h$ here is a charged hadron, typically a pion. "Neutrals" are neutral hadrons, typically $\pi^{0}$ or $K_{L}^{0} \cdot \quad$. 13

2.1 Tevatron Operating parameters for RunI, RunIIa, and RunIIb.

3.1 List of single muon triggers used in the analysis. The field before the first underscore is label for the L1/L2 term, and the field after the first underscore is the L3 term. . . . . . . . . . 35

3.2 List of $\mathrm{Mu}+\mathrm{Tau}(\mathrm{MTA})$ triggers used in the analysis. . . . . . 38

4.1 Muon types and descriptions, based on nseg value[44]. MTC stands for Muon Tracking in the Calorimeter (not used in this analysis.) . . . . . . . . . . . . . 56

4.2 NN b-tagger input variables ranked in order of power.[46] . . 58

5.1 constants in equations 5.1 and 5.2 for each tau type. . . . . . 65

6.1 Breakdown of dataset integrated luminosity by period. The reference trigger used for the luminosity calculation is also given. For the analysis selection, we require the event to fire one of the single muon triggers used in the standard "OR" trigger suite or one of 24 muon+tau triggers. . . . . . . . . . . . . 
6.2 Monte Carlo background samples used in the analysis with the number of generated events after data quality selection, and cross section, and K-factor. For the $W, Z+$ jets and $t \bar{t}$ samples, "excl" means exclusive, or exactly that many additional partons were generated. "Incl" means inclusive, meaning that many or greater. . . . . . . . . . . . . . . .

6.3 Monte Carlo signal samples used in the analysis with the number of generated events after data quality selection, and SM cross section $\times \mathrm{BR}$ in $\mathrm{fb}$ for $\tan \beta=1$. The cross sections come from FEYNHIGGS v2.6.2 [64, 65, 66, 67], and the branching ratios from HDECAY[68]. . . . . . . . . . . . . .

6.4 QCD scaling factors, $N(O S) / N(S S)$ and their statistical errors. We use the type-specific values in the analysis. The average and corresponding $\chi^{2}$ are shown for information only. . . . . . . . . . . . .

6.5 Same-sign (SS) data before $b$-tagging, along with SS MC (sum of all backgrounds.) We subtract the MC from the data, then multiply the result by the $f_{Q C D}$ factors in table 6.4 to get the final pretag QCD result. . . . . . . . . . . .

6.6 Jet to tau fake rates as a function of tau type and their statistical errors. . . . . . . . . . . . . . . . .

6.7 Number of data and background MC events in the noiso sample used in section 6.3.2. We subtract the MC contribution from the data to get the final number for each type. . . . . . . . .

6.8 Final QCD totals for the different methods of estimation. We take the average of the TRF method and Fake Rate method as the overall normalization, and take the QCD shape from the TRF method. . . . . . . . .

6.9 Values of KNN and QCD likelihood cut for each mass point and tau type. . . . . . . . . . . . . . . . .

6.10 Systematic uncertainties applied to each signal and background sample. For shape dependent systematics, denoted as "shape" in the table, we vary the effect by $\pm 1 \sigma$, then use the resulting distributions as inputs to the limit setting program (section 7.1). Those systematics expressed as a percentage are taken as flat systematics (no shape dependence) and are input into the limit setting program accordingly. The dashes ("-") mean that the systematic in that column is not applied to the MC sample in that row. . . . . . . . . . . . . . . . 
6.11 The predicted and observed event yields, with statistical errors, as a function of the selection requirements. We discuss the systematic errors in section 6.5. In this table we assume a signal cross section of $1 \mathrm{pb} \ldots \ldots \ldots \ldots \ldots \ldots$

6.12 Percentage of total events per mass point accepted by each tau type. The signal MC events are generated with inclusive tau decays, not only the $\mu \tau_{\text {had }}$ final state. . . . . . . . . . . 


\section{Acknowledgements}

With any sort of endeavor like this, there are always far too many people to thank than can be thanked in a short space. If you think you deserve recognition, then you do, and I thank you as well.

I must first and foremost thank my advisor, John Hobbs. I have worked with John since the summer of 2003 and I honestly cannot remember ever asking him a question to which he did not know the answer. He has truly been a pleasure to work with in all respects and I cannot say enough good things about him.

I would also like to thank Mike Hildreth of the University of Notre Dame, who was my undergraduate research advisor. He introduced me to the nuts

and bolts of high energy physics and helped me to see that this was something that I seriously wanted to work on.

A number of people working on $\mathrm{D} \varnothing$ have given me advice or assistance with some part of my work there. Among current and former Stony Brook group members I would like to thank Huishi Dong, Satish Desai, Paul Grannis, Jun Guo, Feng Guo, Yuan Hu, Bob McCarthy, Joan Napolitano, Dean Schamberger, Emanuel Strauss, Dmitri Tsybychev, and Junjie Zhu. On the STT side I would like to thank DooKee Cho, Amitabha Das, and David Khatidze. For my trigger work I would like to thank Ike Hall, Marc Hohlfeld, Rick Jesik, Sabine Lammers, Liang Li, Gustavo Otero y Garzon, and Marco Verzocchi. On the analysis side I'd like to thank the Higgs group conveners over the last two years, Gregorio Bernardi, Gavin Davies, Andy Haas, Sasha Khanov, and Krisztian Peters. Also, for various suggestions, pieces of code, and discussions I thank Per Jonsson, Mark Owen, Abid Patwa, Stefan Söldner-Rembold, Peter Svoisky, Marco Verzocchi, and Tammy Yang. Thank you to the members of DØ Editorial Board 18, who review the analysis: Frédérique Badaud, Elemer Nagy, Meenakshi Narain, Flera Rizatdinova (chair), Reinhard Schwienhorst, and Chris Tully. Finally a thanks to the current DØ spokespeople, Dmitri Denisov and Darien Wood, for doing what I am sure is a demanding and often thankless job.

Also a thank you to my thesis committee, consisting of Todd Adams, John 
Hobbs, Bob McCarthy, and Jac Verbaarschot. I must especially thank them for their patience as things have been on a short timescale.

Jadranka Sekaric has been and is very special part of my life, and I can't thank her enough for our time together and everything that she has done for me. I want to say thanks for her support, understanding, love, and patience, especially over the last few months as I've scrambled around to cobble this together. Hvala vram puno, moja najlepša!

I must also thank my parents Rich and Pam, and my sister Emily. They have (literally) been with me every step of the way and have had a most profound influence on my life. I hope that I am always worthy of their love and respect.

Finally to all my friends not listed here: thank you, thank you, thank you. As high-energy physicists we are in search of the most fundamental truths, but at the end of the day it is meaningless without people like you.

Thank you once again to all. 


\section{Chapter 1}

\section{Introduction}

The analysis described herein is a search for neutral Higgs bosons produced in association with a $b$-quark, with the Higgs decaying to a pair of taus. While this process has a very low cross section in the Standard Model (section 1.2) it can be greatly enhanced in supersymmetric models (sec. 1.3). In this chapter we give a general introduction to the theoretical basis of modern elementary particle physics along with some challenges it faces, introduce supersymmetry, and provide a brief overview of what the process of interest would look like from an experimental point of view. ${ }^{1}$

\subsection{The Fundamental Forces and Particles}

The 20th century saw a revolution in our understanding of the universe. From relativity to quantum mechanics to field theory, a significant change in our thinking has taken place. We now know that there are three fundamental forces in nature, the strong force, the electroweak force, and gravity, each with its own strength and effective range. Furthermore, these forces can be described in terms of fields, both vector and scalar. The forces are quantized, meaning that they occur in discrete amounts, and that each force has a particle or particles (the quanta) that act as a mediator of that force.

To the best of our knowledge everything in the universe consists of bosons and fermions at the most fundamental level. Fermions are 1/2-integer spin particles that make up ordinary matter, called fermions because they obey Fermi-Dirac statistics. Bosons are integer spin particles that mediate interac-

\footnotetext{
${ }^{1}$ Throughout this dissertation we will use "natural", or Heaviside-Lorentz, units, where $\hbar=c=1$. Thus momentum and energy are both expressed in terms of electron volts $(\mathrm{eV})$, with $1 \mathrm{GeV}=10^{9} \mathrm{eV}$. In SI units, momentum can be expressed as $\mathrm{GeV} / c$, and energy as $\mathrm{GeV} / c^{2}$.
} 
tions between particles (both fermions and other bosons), so named because they obey Bose-Einstein statistics. Fermions can be further classified into quarks and leptons. Quarks (and antiquarks) carry a $\pm 2 / 3$ or $\mp 1 / 3$ electric charge, have masses ranging from less than $1 \mathrm{GeV}$ up to $\approx 175 \mathrm{GeV}$, and interact via the strong and electroweak interactions. There are six quarks: up, down, charm, strange, top, and bottom, arranged in three generations, or families. Table 1.1 lists their masses and charge. Quarks form composite particles known as hadrons. Hadrons are either mesons, consisting of one quark and one antiquark, or baryons, consisting of three quarks (or three antiquarks for antibaryons), the best known of which are the proton and neutron. Leptons are fermions that do not interact via the strong force, only via the electroweak force. There are six leptons, also arranged in three families: the electron, the electron neutrino, the muon, the muon neutrino, the tau, and the tau neutrino. Neutrinos are charge 0 particles that do not feel the electromagnetic force and until recently were treated as massless. The charged leptons have charge -1 (antileptons have a +1 charge), and their properties are also in Table 1.1.

The strong force has the greatest intrinsic strength, but is limited to a range of roughly the size of an atomic nucleus. Its best known effect is to bind the atomic nucleus together, but it also plays a significant role in particle production and decay. Its force carrier is the gluon, a massless spin 1 particle. The quantum number for the strong force is color; color can either be red, blue, or green. A (anti)quark carries one unit of (anti)color, while a gluon carries one unit of color and one unit of anticolor. The strong force obeys an $S U(3)$ symmetry, with the eight generators of the $S U(3)$ group corresponding to the eight gluons. The electroweak force was once thought to be two separate forces: the weak force, responsible for nuclear $\beta$ decay, among other things, and the electromagnetic force that plays a role in everyday life. We now know that the force carriers are the $W^{+}, W^{-}$, and $Z$ bosons, and the photon $(\gamma)$. The electroweak force obeys an $S U(2)_{L} \otimes U(1)_{Y}$ symmetry. The $S U(2)_{L}$, (i.e. the weak) component of the electroweak forces couples only to left-handed fermions; this explains why parity symmetry is not conserved (in fact, is maximally violated) in a weak interaction. It also has a limited range, while the electromagnetic component has infinite range.

The familiar gravitational force is also one of the three fundamental forces, but its intrinsic strength is so much smaller than the other two forces (about $10^{-42}$ that of the strong force) that it is completely negligible here. Its force carrier is the graviton, which has not been directly observed.

With the introduction of the forces, we have a particle family of six quarks, six leptons, four force carriers, along with their corresponding antiparticles. 


\begin{tabular}{|c|c|c|c|c|c|c|c|c|}
\hline & \multicolumn{8}{|c|}{ Fermions } \\
\hline & \multicolumn{4}{|c|}{ Quarks } & \multicolumn{4}{|c|}{ Leptons } \\
\hline Family & Name & $q$ & Mass $(\mathrm{GeV})$ & Forces & Name & $q$ & Mass $(\mathrm{GeV})$ & Forces \\
\hline 1 & $u$ & $+2 / 3$ & $1.5-3.3 \times 10^{-3}$ & S,E,W & $\nu_{e}$ & 0 & $\approx 0$ & W \\
\hline 1 & $d$ & $-1 / 3$ & $3.5-6.0 \times 10^{-3}$ & S,E,W & $e$ & -1 & $5.11 \times 10^{-4}$ & $\mathrm{E}, \mathrm{W}$ \\
\hline 2 & $c$ & $+2 / 3$ & 1.27 & $\overline{\mathrm{S}, \mathrm{E}, \mathrm{W}}$ & $\nu_{\mu}$ & 0 & $\approx 0$ & $\bar{W}$ \\
\hline 2 & $s$ & $-1 / 3$ & 0.104 & $\mathrm{~S}, \mathrm{E}, \mathrm{W}$ & $\mu$ & -1 & 0.105 & $\mathrm{E}, \mathrm{W}$ \\
\hline 3 & $t$ & $+2 / 3$ & 171.2 & S,E,W & $\nu_{\tau}$ & 0 & $\approx 0$ & W \\
\hline \multirow[t]{7}{*}{3} & $b$ & $-1 / 3$ & 4.2 & S,E,W & $\tau$ & -1 & 1.77 & $\mathrm{E}, \mathrm{W}$ \\
\hline & \multicolumn{8}{|c|}{ Bosons } \\
\hline & \multicolumn{2}{|c|}{ Name } & \multicolumn{2}{|l|}{$q$} & \multicolumn{2}{|c|}{ Mass $(\mathrm{GeV})$} & \multicolumn{2}{|c|}{ Force Mediated } \\
\hline & \multirow{4}{*}{\multicolumn{2}{|c|}{$\begin{array}{c}g \\
\gamma \\
W^{ \pm} \\
7\end{array}$}} & \multicolumn{2}{|l|}{0} & \multicolumn{2}{|c|}{0} & \multicolumn{2}{|l|}{$\mathrm{S}$} \\
\hline & & & \multicolumn{2}{|l|}{0} & \multicolumn{2}{|c|}{0} & \multicolumn{2}{|l|}{$\mathrm{E}$} \\
\hline & & & \multicolumn{2}{|l|}{ \pm 1} & \multicolumn{2}{|c|}{80.4} & \multicolumn{2}{|l|}{$\mathrm{W}$} \\
\hline & & & \multicolumn{2}{|l|}{0} & \multicolumn{2}{|c|}{91.2} & \multicolumn{2}{|l|}{$\mathrm{W}$} \\
\hline
\end{tabular}

Table 1.1: List of the elementary particles and force carriers, including their electromagnetic charge $q$, masses in $\mathrm{GeV}$, and what forces they feel in the case of fermions, or mediate in the case of bosons. $\mathrm{S}$ is the strong force, $\mathrm{E}$ is the electromagnetic part of the electroweak force, and $\mathrm{W}$ is the weak part of the electroweak force. The fermions are also grouped in their three generations, or families. Masses taken from [1]. Gravity is neglected here.

\subsection{The Standard Model}

Over the last few decades the Standard Model (SM) has emerged as the effective field theory up to at least the TeV scale. It combines the strong and electroweak forces into a framework that governs all interactions of elementary particles. It does not incorporate gravity, but gravity is so weak relative to the other forces that it plays no role at the subatomic level. Below we discuss the details of the standard model and its phenomenology.

\subsubsection{Formalism}

We begin with the Strong force, which obeys an $S U(3)$ symmetry. It governs the interactions between quarks and gluons. The Lagrangian for the Strong force is $[2]$

$$
\mathcal{L}=\bar{\psi}\left(i \gamma^{\mu} \mathcal{D}_{\mu}-m\right) \psi-\frac{1}{2} \operatorname{tr}\left(G_{\mu \nu} G^{\mu \nu}\right)
$$

where $\psi$ is a composite spinor of the color triplet, $\mathcal{D}_{\mu}$ is the covariant derivative $\mathcal{D}_{\mu}=\partial_{\mu}+i g B_{\mu}$, and $B_{\mu}$ is a $3 \times 3$ matrix formed from the eight color gauge 
fields (corresponding to the eight gluons) and generators of the $S U(3)$ group.

The electromagnetic component of the electroweak force obeys $U(1)$ symmetry, meaning it is invariant under hypercharge $(Y)$ transformations. It has only one force carrier, the photon. The weak component is invariant under weak isospin transformations. Weak isospin and hypercharge are related to the familiar electric charge by $Q=T_{3}+Y / 2$ where $T_{3}$ is the third component of weak isospin. We can write the combined electroweak Lagrangian (electron sector only) as [2]:

$$
\begin{aligned}
\mathcal{L}= & -\frac{1}{4} B^{\mu \nu} B_{\mu \nu}-\frac{1}{4} W^{i, \mu \nu} W_{\mu \nu}^{i}+i \bar{e}_{R} \gamma^{\mu}\left(\partial_{\mu}+i g^{\prime} \frac{Y}{2} B_{\mu}\right) e_{R} \\
& +i \bar{l}_{L} \gamma^{\mu}\left(\partial_{\mu}+i g^{\prime} \frac{Y}{2} B_{\mu}+i g \tau^{i} W_{\mu}\right) l_{L}
\end{aligned}
$$

Where $Y$ is the hypercharge operator, $e_{R}$ is the right-handed Dirac spinor for the electron, and $B_{\mu \nu}$ and $W_{\mu \nu}$ are the field strength tensors corresponding to two vector fields $B$ and $W$. Thus we have the free Lagrangian for $B_{\mu}$ and $W_{\mu}^{i}$ :

$$
\begin{aligned}
B_{\mu \nu} & =\partial_{\mu} B_{\nu}-\partial_{\nu} B_{\mu} \\
W_{\mu \nu}^{i} & =\partial_{\mu} W_{\nu}^{i}-\partial_{n u} W_{\mu}^{i}+g \epsilon^{i j k} W_{\mu}^{j} W_{\nu}^{k}
\end{aligned}
$$

We can rewrite the Lagrangian in equation in terms of the photon $\left(A_{\mu}\right)$, charged $\mathrm{W}\left(W^{ \pm}\right)$fields, and the $Z\left(Z_{\mu}\right)$ field [2]:

$$
\begin{aligned}
W_{\mu}^{ \pm} & =\frac{1}{\sqrt{2}}\left(W_{\mu}^{1} \mp i W_{\mu}^{2}\right) \\
A_{\mu} & =\cos \theta_{W} B_{\mu}+\sin \theta_{W} W_{\mu}^{3} \\
Z_{\mu} & =-\sin \theta_{W} B_{\mu}+\cos \theta_{W} W_{\mu}^{3}
\end{aligned}
$$

where $\theta_{W}$ is the weak mixing angle, or Weinberg angle.

Finally we have a combined theory that obeys $S U(3)_{c} \otimes S U(2)_{L} \otimes U(1)_{Y}$ symmetry and accounts for the strong and electroweak forces as well as all particles observed in nature thus far. Additionally, we know that electroweak $S U(2)_{L} \otimes U(1)_{Y}$ symmetry is not an exact symmetry of nature; it is spontaneously broken at some energy scale of order $1 \mathrm{TeV}$. After spontaneously breaking electroweak symmetry we have massless vector bosons and no fermion mass terms, plainly contradicting empirical evidence. A solution comes in the form of the Higgs mechanism [3], the application of local gauge invariance to the spontaneously broken symmetry. 


\subsubsection{The Higgs Mechanism}

If we define $\phi$ as a complex field consisting of the two real fields:

$$
\phi \equiv \frac{\phi_{1}+i \phi_{2}}{\sqrt{2}}
$$

then using covariant derivatives

$$
\mathcal{D} \equiv \partial_{\mu}+i q A_{\mu}
$$

we have the Lagrangian for electrodynamics of charged scalar particles :

$$
\mathcal{L}=\left|\mathcal{D}^{\mu} \phi\right|^{2}-\mu^{2}|\phi|^{2}+|\lambda|\left(\phi^{\dagger} \phi\right)^{2}-\frac{1}{4} F_{\mu \nu} F^{\mu \nu}
$$

Applying the principle of local gauge invariance, this Lagrangian is invariant under local gauge transformations:

$$
\begin{aligned}
\phi(x) \rightarrow \phi^{\prime}(x) & =e^{i q \alpha x} \phi(x), \\
A_{\mu}(x) \rightarrow A_{\mu}^{\prime}(x) & =A_{\mu}(x)-\partial_{\mu} \alpha(x)
\end{aligned}
$$

If $-|\mu|^{2}<0$, the symmetry is broken. The potential then has a minimum at

$$
\left\langle|\phi|^{2}\right\rangle_{0}=v^{2} / 2
$$

We can then shift the field so that we have

$$
\langle\phi\rangle_{0}=v / 2
$$

and thence define the shifted field $\phi \prime$ as

$$
\phi^{\prime}=\phi-\langle\phi\rangle_{0}
$$

Then we can introduce two new real fields $\eta$ and $\zeta$ :

$$
\eta=\phi_{1}-\mu / \lambda, \zeta=\phi_{2}
$$

$\phi$ can then be written as

$$
\phi=e^{i \zeta / v}(v+\eta) / \sqrt{2} \approx(v+\eta+i \zeta) / \sqrt{2}
$$

then we can rewrite the local gauge transformation $\phi \rightarrow \phi^{\prime}$ as:

$$
\phi \rightarrow \phi^{\prime}=e^{-i \zeta(x) / v} \phi(x)=(v+\eta) / \sqrt{2}
$$


We can also choose a convenient gauge such that the $A_{\mu}(x) \rightarrow A_{\mu} \prime(x)$ transformation is:

$$
A_{\mu}(x) \rightarrow A_{\mu}^{\prime}(x)=A_{\mu}(x)+\frac{1}{q v} \partial_{\mu} \zeta .
$$

Finally we can substitute these transformations we obtain

$$
\mathcal{L}=\frac{1}{2}\left[\left(\partial_{\mu} \eta\right)\left(\partial^{\mu} \eta\right)+2 \mu^{2} \eta^{2}\right]-\frac{1}{4} F_{\mu \nu} F^{\mu \nu}+\frac{q^{2} v^{2}}{2} A_{\mu}^{\prime} A^{\mu \prime}
$$

Now the physical states arising from this Lagrangian are clear. We have a scalar field $\eta$ with a mass squared $-\mu^{2}>0$; a massive vector field $A_{\mu}$, with a mass $q v$, and the $\zeta$ field has disappeared thanks to the choice of gauge. In terms of a particle spectrum, instead of four massless vector bosons after electroweak symmetry breaking, we have three massive vector bosons (the $W^{ \pm}$and $Z$ ), and one massive scalar, the Higgs boson. As the system is still invariant under $U(1)$ symmetry the photon remains massless. One can imagine that the vector field has "eaten" the massless Goldstone boson, thereby giving the vector field mass and a third (longitudinal) polarization state. Now we have a theory that correctly describes what we see in nature (the fermion mass terms are generated through Yukawa couplings to the Higgs boson [4]), and introduces one new particle, which, despite intensive searches at LEP and the Tevatron, has yet to be seen experimentally. LEP searches have established a lower limit on the SM Higgs mass of $114.4 \mathrm{GeV}$ [5] , and the Tevatron has also excluded a mass of $170 \mathrm{GeV}$ at the $95 \%$ confidence level [6].

\subsubsection{Challenges to the Standard Model}

Despite the Standard Model's extensive success to this point, it does face a number of challenges. The most pertinent to this analysis is perhaps the socalled hierarchy problem. If one computes higher-order corrections to the Higgs mass term coming from top quark loops, one finds terms that are quadratically and logarithmically divergent [7]. That is, as one increases the energy scale, the Higgs mass approaches infinity, absent extreme fine-tuning (nearly 40 decimal places) at all orders. Fine-tuning is generally deemed a poor solution, thus some other solution is needed to keep the Higgs mass finite. There is also the triviality problem, which is that renormalizing the Higgs self-coupling sets it to 0 at some energy scale $\Lambda$ of order $1 \mathrm{TeV}$. Another challenge concerns the relative force strength at extremely high energies, approaching the Planck scale $\left(10^{19} \mathrm{GeV}\right)$. In a truly unified theory the couplings should come to a common point at some energy scale. In the Standard Model, the force strengths never 
intersect at a common point, as shown in figure 1.1.

\subsection{Supersymmetry}

Supersymmetry (SUSY) is a symmetry between fermions and bosons. It creates a new particle, or superpartner, for each particle in the SM. The superpartners differ from their standard model counterparts in spin and mass. A standard model fermion's superpartner will have integer spin, while a boson's superpartner will have half-integer spin. While exact supersymmetry would mean the superpartners would have identical mass to their standard model counterparts, no superpartner has been observed experimentally, implying that supersymmetry is broken and the superpartners are much more massive than the Standard Model particles. One can find reviews of supersymmetry in [8],[9],[10].

Supersymmetry is able to answer the challenges to the standard model in section 1.2.3. The SUSY Lagrangian introduces new corrections to the Higgs mass term that neatly cancel the divergent terms [7]. Additionally, the triviality problem disappears. Further, if one evaluates the inverse of the running coupling constants for the $S U(3)$ (strong), $S U(2)$ (weak), and $U(1)$ (electromagnetic) forces, they do converge to a common point when plotted as a function of the energy scale after including sparticle corrections as shown on the right side of figure 1.1. SUSY may also be an attractive solution to the question of dark matter. In supersymmetric theories with $R$-parity conservation $\left(R=(-1)^{2 j+3 B+L}\right.$, where $j$ is spin, $B$ is baryon number, and $L$ is lepton number), sparticles will always eventually decay to an odd number of superpartners, the lightest of which (LSP) is absolutely stable. The lightest superpartner would then be a candidate for dark matter.

\subsubsection{MSSM}

The simplest form of supersymmetry is the Minimal Supersymmetric Standard Model (MSSM) [7, 8, 9]. The MSSM assigns a vector field to a vector supermultiplet, and the matter fields to chiral supermultiplets. The resulting particle content is a new partner for each standard model particle differing by mass and by one-half integer in spin. Thus each quark has a superpartner called a squark, each lepton a superpartner called a slepton, and the gauge bosons have spin $\frac{1}{2}$ superpartners called the gluino, photino, wino, and zino. We discuss the Higgs sector in the next section. In the MSSM supersymmetry is broken "softly" by a "hidden sector" of the theory that does not directly couple to the standard model fermions and gauge bosons [8]. It results in a 


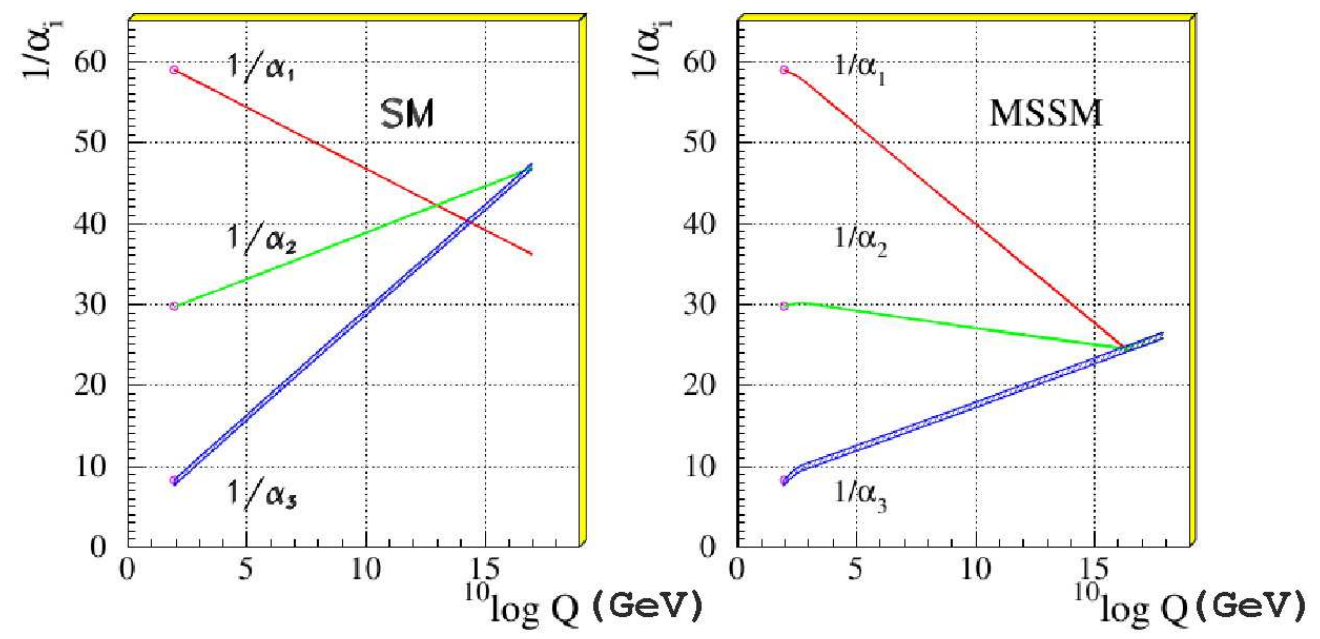

Figure 1.1: Relative coupling constants of the three gauge symmetries as a function of energy in the Standard Model (left) and the MSSM (right). We see that the couplings converge to a common point in the MSSM [11].

phenomenologically acceptable picture.

\subsubsection{Higgs Phenomenology in the MSSM}

In the MSSM there are two Higgs doublets, one with hypercharge $Y=+1$, $\Phi_{d}=\left(\Phi_{d}^{0}, \Phi_{d}^{-}\right)$, and one with hypercharge $Y=-1, \Phi_{u}=\left(\Phi_{u}^{+}, \Phi_{u}^{0}\right) . \Phi_{u}^{0}$ and $\Phi_{d}^{0}$ couple exclusively to up and down-type fermions, respectively. After minimizing the Higgs potential the neutral components of the Higgs fields acquire the vacuum expectation values

$$
\left\langle\Phi_{d}\right\rangle=\frac{1}{\sqrt{2}}\left(\begin{array}{c}
v_{d} \\
0
\end{array}\right),\left\langle\Phi_{u}\right\rangle=\frac{1}{\sqrt{2}}\left(\begin{array}{c}
0 \\
v_{u}
\end{array}\right)
$$

with the normalization chosen so that $v_{d}^{2}+v_{u}^{2}=4 m_{w}^{2} / g^{2}=(246 \mathrm{GeV})^{2}$.

The two doublets have eight total degrees of scalar freedom. As in the SM case, EWSB provides the longitudinal modes of the $W$ and $Z$ bosons. Now, instead of one remaining physical Higgs boson, there are five (since there are now eight degrees of freedom instead of four.) There are two charged Higgs bosons, $H^{ \pm}$, and three neutral Higgs bosons: a $C P$-odd pseudoscalar $A$, and two $C P$-even scalars $h$ and $H$, with $m_{h} \leq m_{H}$. The structure of the theory is such that all tree-level Higgs sector parameters can be expressed in terms of the electroweak gauge coupling constants and two free parameters: the ratio 
of the vacuum expectation values of the two doublets,

$$
\tan \beta \equiv \frac{v_{u}}{v_{d}}
$$

and one of the Higgs masses, typically $m_{A}$. Thus we can find the tree-level masses of the other Higgs bosons:

$$
\begin{aligned}
& m_{H^{ \pm}}^{2}=m_{A}^{2}+m_{W}^{2} \\
& m_{H, h}^{2}=\frac{1}{2}\left(m_{A}^{2}+m_{Z}^{2} \pm \sqrt{\left(m_{A}^{2}+m_{Z}^{2}\right)^{2}-4 m_{A}^{2} m_{Z}^{2} \cos ^{2} 2 \beta}\right)
\end{aligned}
$$

The Higgs couplings to fermions, namely down-type fermions, is enhanced relative to the standard model. The neutral Higgs couplings to third-generation down-type fermions relative to their standard model values $g m_{f} / 2 m_{w}$ are [12]:

$$
\begin{aligned}
h b \bar{b}\left(o r h \tau^{+} \tau^{-}\right) & : \sin (\beta-\alpha)-\tan \beta \cos (\beta-\alpha) \\
H b \bar{b}\left(\text { or } H \tau^{+} \tau^{-}\right) & : \cos (\beta-\alpha)+\tan \beta \sin (\beta-\alpha) \\
A b \bar{b}\left(\text { or } A \tau^{+} \tau^{-}\right) & : \gamma^{5} \tan \beta
\end{aligned}
$$

where $\alpha$ is the angle that diagonalizes the Higgs mass-squared matrix. Thus at large $\tan \beta$ the couplings can be significantly enhanced. Additional radiative corrections in the form of loop effects can also modify the couplings by introducing corrections to the fermion mass terms [12].

\section{Neutral Higgs production at the Tevatron}

The leading process for $\phi b^{2}$ production at the Tevatron is $g b \rightarrow \phi b$, with the initial state $b$-quark coming from the proton sea (usually created from gluon splitting.) Figure 1.2 illustrates the tree-level Feynman diagrams for this process. The cross section for $h b$ production has been calculated explicitly at next-to-leading order (NLO) in the standard model [13]. Figure 1.3 shows the production cross section as a function of Higgs mass. The cross section for the charge conjugate process $g \bar{b} \rightarrow \phi \bar{b}$ is identical. In the MSSM, however, we have seen that the Higgs coupling to fermions is enhanced by a factor of order $\tan \beta$, meaning the production cross section (a function of the coupling squared) is enhanced by $\tan ^{2} \beta$. For a sufficiently high $\tan \beta$ the cross section will be enhanced to the level of several pb. Furthermore, for any pseudoscalar Higgs mass is below $200 \mathrm{GeV}$, one of the scalar Higgs bosons is nearly degenerate

\footnotetext{
${ }^{2}$ Here and throughout this dissertation $\phi$ refers to the set of three neutral Higgs bosons.
} 
with the pseudoscalar. Thus the total effective cross section is doubled, leading to a total production cross section enhancement of $2 \tan ^{2} \beta$.
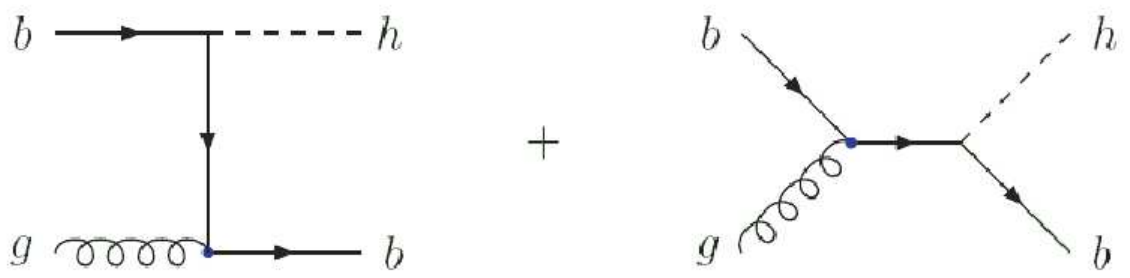

Figure 1.2: Tree-level diagrams of $h b$ production. There are similar diagrams for $A b$ and $H b$ production.

\section{Higgs decay modes}

Decays to fermion pairs dominate neutral Higgs decays for masses below several hundred GeV. As the Higgs couples to mass, the Higgs will preferentially decay to the most massive particles kinematically available (however, decays to diboson pairs are suppressed.) Neutral Higgs bosons will decay to $b \bar{b}$ pairs approximately $90 \%$ of the time, with $\tau \tau$ pair decays making up about nine of the remaining ten percent. Figures 1.4 and 1.5 show the Higgs decay modes and branching ratios for each of the neutral Higgs bosons at $\tan \beta=30$. Depending on the particular Higgs sector and MSSM parameters the Higgs masses can be sensitive to third-generation squark masses due to radiative corrections.

\subsection{Experimental Signatures}

As we have seen, the SM cross section for the $\phi b$ process is only a few $\mathrm{fb}$, but in the MSSM if $\tan \beta$ is sufficiently large the cross section can be enhanced to several pb, making it accessible at the Tevatron. From a purely theoretical point of view, $\phi b \rightarrow b b \bar{b}$ is the best single channel to search for $\phi b$ production due to the large branching ratio. However, the $b b \bar{b}$ final state suffers from a large multijet background making it very difficult to distinguish the signal. Nevertheless, $b b \bar{b}$ searches have been performed at the Tevatron [14],[15],[16],[17]. A final state where the Higgs decays to taus offers a smaller multijet background and allows for easier object identification.

Tau branching ratios are summarized in Table 1.2. The maximum branching ratio in a single channel comes from requiring both taus to decay hadronically, leading to a $\tau_{h} \tau_{h} b$ final state. However, hadronic tau identification is more difficult then lepton identification at D $\varnothing$, plus the multijet background, 


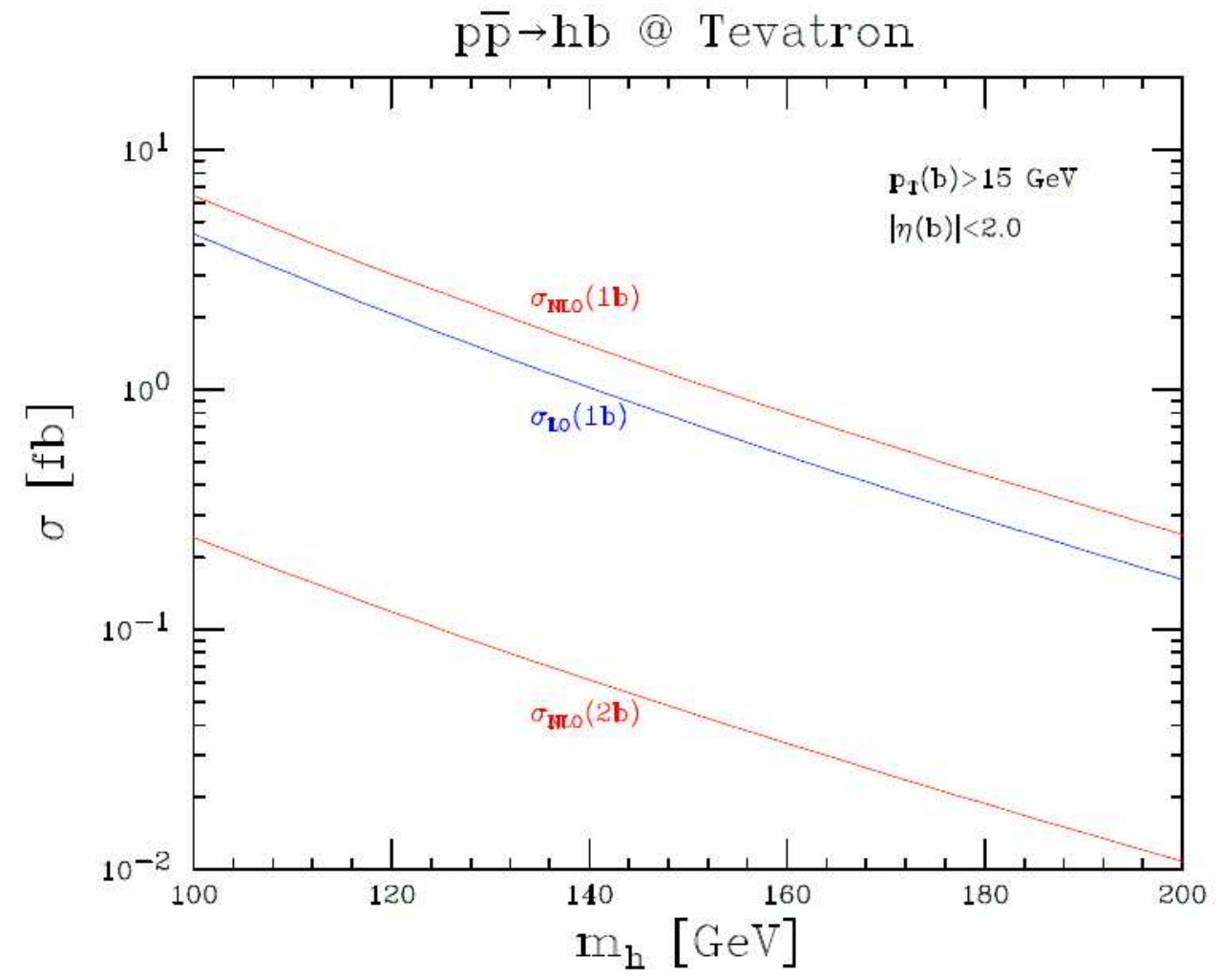

Figure 1.3: Standard model $\phi b$ production cross section in fb at the Tevatron. The associated $b$-quark has transverse momentum, or $p_{T}$, grater than $15 \mathrm{GeV}$ and $|\eta|<2.0[13] . \eta=-\ln \left(\tan \frac{\theta}{2}\right)$ (Sec.2.2.1). In the MSSM, the production cross sections can be significantly enhanced over these shown here by a factor of order $\tan ^{2} \beta$. 


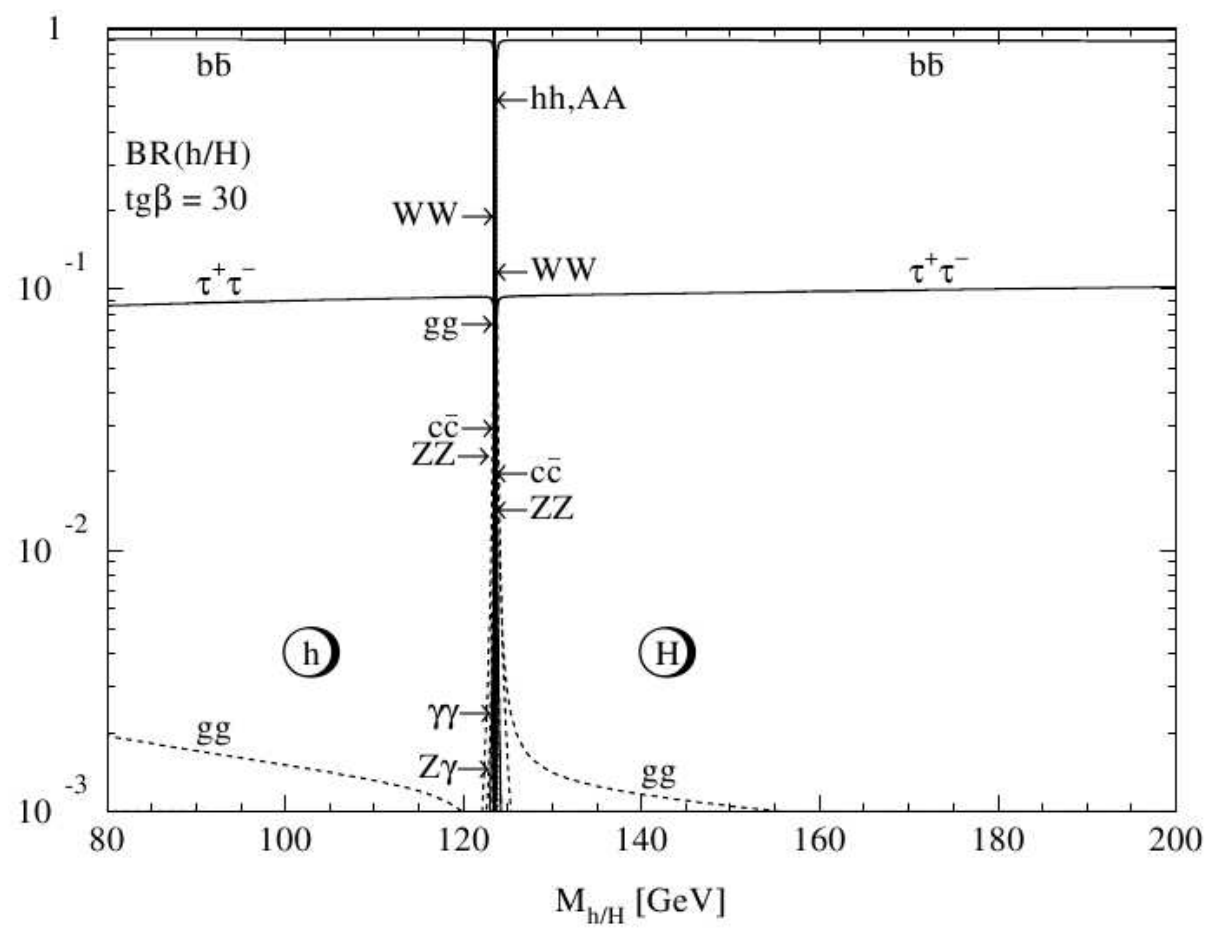

Figure 1.4: Branching ratios of the scalar MSSM Higgs bosons $h$ and $H$ at $\tan \beta=30[12]$. 


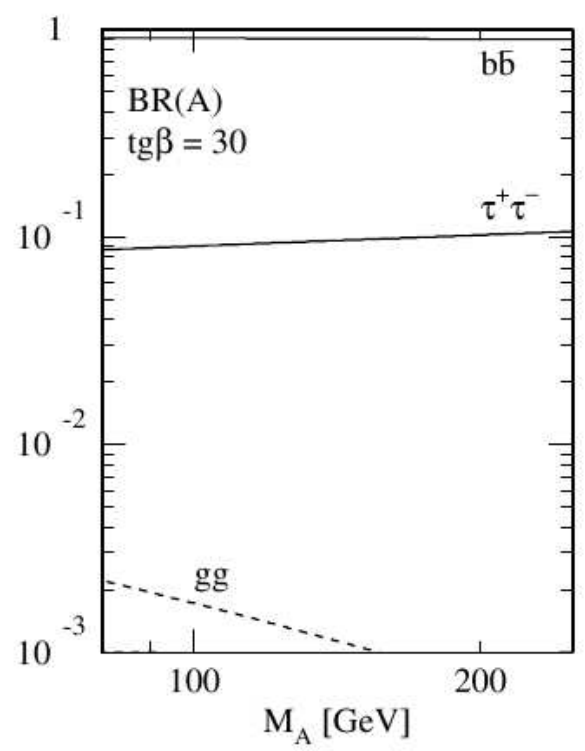

Figure 1.5: Branching ratios of the pseudoscalar MSSM Higgs boson $A$ at $\tan \beta=30[12]$.

while much smaller than the $b b \bar{b}$ final state, has not been rigorously studied to date. On the other hand the di-lepton final states suffer from a low overall branching ratio. A final state with one hadronic tau and one lepton, a muon in our case, with a branching ratio of $\approx 23 \%$, offers a good balance between branching ratio and object identification. Thus the signature in the detector is a hadronic tau, an isolated muon (isolated from other jets and tracks) and a jet that is consistent with a $b$-quark. We will discuss identification of these objects in Chapter 4 . There has been one previous search in this channel done at $\mathrm{D} \varnothing[18]$.

\begin{tabular}{|l|c|}
\hline Mode & Branching Ratio \\
\hline$\mu \bar{\nu}_{\mu} \nu_{\tau}$ & $(17.36 \pm 0.05) \%$ \\
$e \bar{\nu}_{e} \nu_{\tau}$ & $(17.85 \pm 0.05) \%$ \\
$h \nu_{\tau}$ & $(12.13 \pm 0.07) \%$ \\
$h \nu_{\tau}+\geq 1$ neutrals & $(37.08 \pm 0.11) \%$ \\
$h^{-} h^{+} h^{-} \nu_{\tau}+\geq 0$ neutrals & $(15.19 \pm 0.08) \%$ \\
\hline
\end{tabular}

Table 1.2: Tau branching ratios [1]. $h$ here is a charged hadron, typically a pion. "Neutrals" are neutral hadrons, typically $\pi^{0}$ or $K_{L}^{0}$. 


\section{Chapter 2}

\section{Experimental Apparatus}

This analysis uses data taken at the $\mathrm{D} \varnothing$ detector, one of two general-purpose detectors at the Fermilab Tevatron. In this chapter we give an overview of Fermilab, the Tevatron and associated accelerator chain, and then provide a description of the $\mathrm{D} \varnothing$ detector.

\subsection{Fermi National Accelerator Laboratory}

Fermi National Accelerator Laboratory, or Fermilab, in Batavia, Illinois, began in 1967 as the National Accelerator Laboratory (NAL). The name changed in 1974 and in the course of its history Fermilab has been home to a number of momentous discoveries in high-energy physics, including first evidence for the bottom quark, and discovery of the top quark and tau neutrino [19]. Today Fermilab has active programs in hadron collider physics, neutrino physics, fixed-target experiments, theory, and astrophysics. The centerpiece of the hadron collider physics program is the Tevatron, a proton-anti-proton $(p \bar{p})$ collider that operates at a center-of-mass energy of $1.96 \mathrm{TeV}$; it can also be used for fixed-target experiments. In the next two sections we detail the accelerator chain leading up to the Tevatron and describe its operation.

\subsubsection{Accelerator Complex}

Figure 2.1 depicts the layout of the accelerator chain at Fermilab. The accelerator chain begins with the pre-accelerator, which takes hydrogen gas and strips it of electrons using a magnetron surface-plasma source. Some of the positive ions collect two electrons, become $\mathrm{H}^{-}$ions and attain a kinetic energy of $18 \mathrm{keV}$ and are extracted from the magnetron by an extractor plate kept at $18 \mathrm{kV}$. The next stage is the Cockroft-Walton accelerator, which acceler- 
ates the $18 \mathrm{keV} \mathrm{H}^{-}$ions up to $750 \mathrm{keV}$. The beam then moves to the Linear Accelerator (LINAC). The LINAC has two functions: to accelerate the beam from $750 \mathrm{keV}$ to $400 \mathrm{MeV}$, and to change the beam from a continuous stream of particles into pulses, or "bunches", of particles, with $201.25 \mathrm{MHz}$ frequency, or 201.25 million pulses per second.

The beam goes to a debuncher to reduce the momentum spread in the bunches, and then passes through carbon foils that strip off electrons, leaving a proton-only beam. The beam then moves to the Booster, a $75.5 \mathrm{~m}$ radius synchroton accelerator. The Booster uses RF fields and varying magnetic fields to accelerate bunches to $8 \mathrm{GeV}$. After the Booster the proton beam passes to the Main Injector, a larger synchroton that accelerates the proton bunches to $150 \mathrm{GeV}$. The Main Injector can then feed protons to the Tevatron or send $120 \mathrm{GeV}$ protons to the anti-proton source.

The anti-proton source consists of a nickel target, collection lens, Debuncher, and Accumulator. $120 \mathrm{GeV}$ protons from the Main Injector strike the target, producing a shower of secondary particles, including anti-protons (pbars). Making anti-protons is notoriously inefficient. It typically takes $10^{5}$ protons to produce one or two pbars. The secondary particles impinge on a lithium collection lens that collects and focuses negatively charged particles, and then a dipole bending magnet extracts the pbars to the debuncher. The anti-protons are produced with random momenta. The Debuncher reduces the spread and creates a collection of pbars with uniform momentum to send to the Accumulator. The Accumulator creates a bunch structure for the pbars similar to that of the protons and is able to store a large number of pbars before accelerating them to $8 \mathrm{GeV}$ and sending them to the Main Injector, where they are accelerated to $150 \mathrm{GeV}$, but travel in the opposite direction of the protons. From the Main Injector both protons and anti-protons go to the Tevatron. 


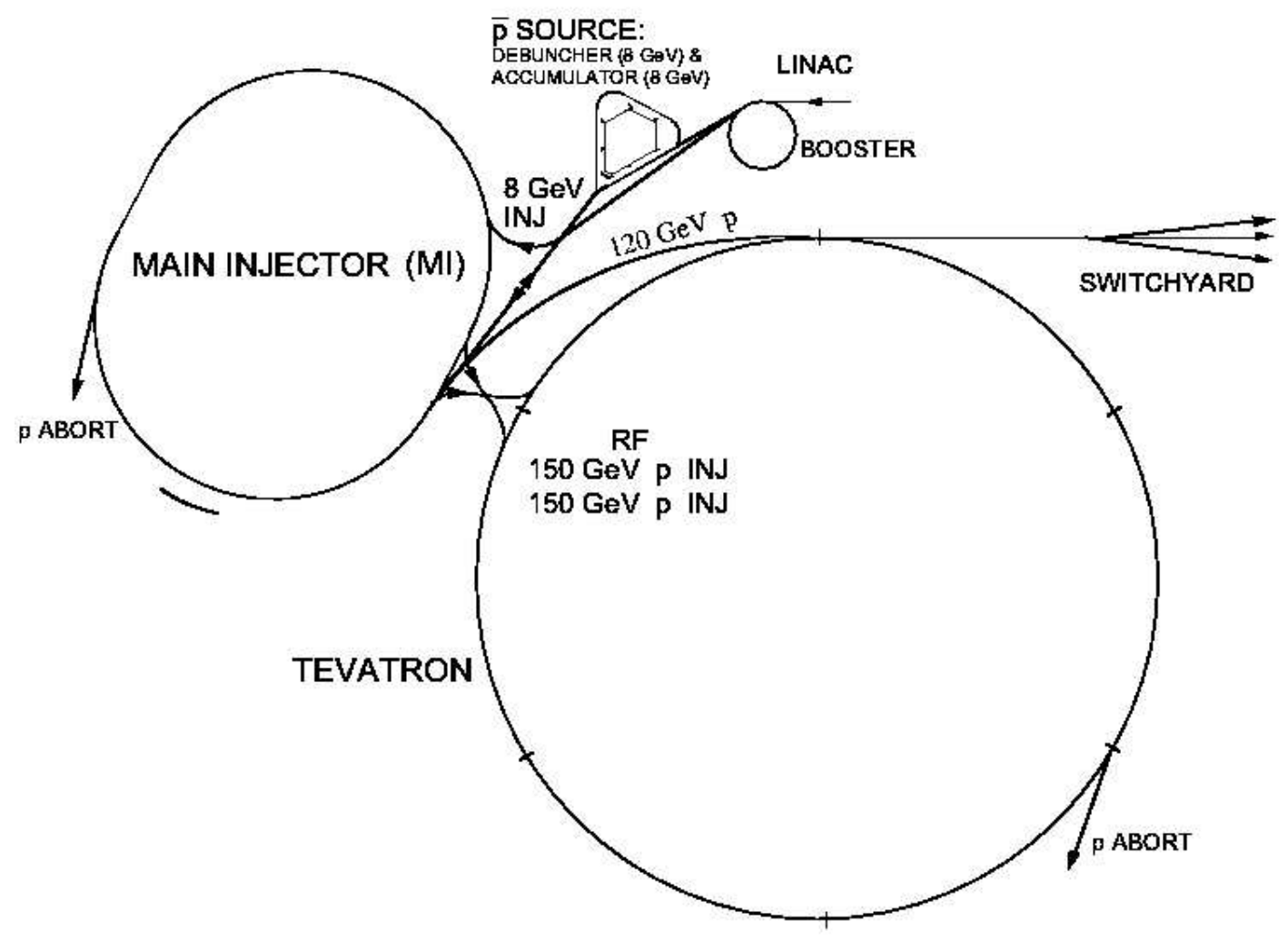

Figure 2.1: Overview of Fermilab, the Tevatron, and the associated accelerators. The Tevatron has a $1 \mathrm{~km}$ radius; everything is drawn to the appropriate scale. [20]. 


\subsubsection{The Tevatron}

The final stage of the accelerator is the Tevatron, a $1 \mathrm{~km}$ radius synchroton ring. The electromagnetic structure has 1,113 RF buckets and approximately 1,000 superconducting magnets used to keep the proton and anti-proton beams in orbit. The magnets produce fields of $\approx 4 \mathrm{~T}$ and operate at liquid helium temperatures. The beams travel in the same tunnel in opposite directions with a helical beam shape, and can be brought together in collision by means of low$\beta$ quadrupole magnets at the $\mathrm{B} \varnothing$ and $\mathrm{D} \varnothing$ interaction regions, located inside the CDF and D $\varnothing$ detectors, respectively. The beam spot size in the transverse plane at collision is less than $50 \mu \mathrm{m}$ in diameter. During collisions there are 36 bunches of protons and anti-protons in the Tevatron, with $\approx 3 \times 10^{11}$ protons per bunch and $6-10 \times 10^{10}$ anti-protons per bunch.

The unit of time in the Tevatron is the tick, or one cycle of the Tevatron clock, which is 132 ns. p $\bar{p}$ bunches are grouped into three groups of 12, known as superbunches. The spacing between bunches within each superbunch is three ticks, or $396 \mathrm{~ns}$. There is a 20 tick separation between the superbunches, thus a bunch will make one full revolution in the Tevatron every 159 ticks.

There have been three major running periods for the Tevatron Collider: RunI (1991-1996), RunIIa (2001-2006), and RunIIb (2006-present.) Table 2.1 summarizes the Tevatron typical operating parameters for RunI, RunIIa, and RunIIb.

The instantaneous luminosity in the Tevatron can be written as

$$
\mathcal{L}=\frac{N_{p} N_{\bar{p}} n_{B} f}{2 \pi\left(\sigma_{p}^{2}+\sigma_{\bar{p}}^{2}\right)} F\left(\sigma_{l} / \beta^{*}\right)
$$

where $N_{p}$ and $N_{\bar{p}}$ are the number of protons and pbars per bunch, respectively, $n_{B}$ is the number of bunches (36), $f$ is the bunch revolution frequency, $\sigma_{p}$ and $\sigma_{\bar{p}}$ are the transverse sizes of the proton and anti-proton beams at the collision point, and $F\left(\sigma_{l} / \beta^{*}\right)$ is the form factor that depends on the bunch length $\sigma_{l}$ and $\beta^{*}$. Peak instantaneous luminosities at the Tevatron are now above $3 \times 10^{32} \mathrm{~cm}^{-2} \mathrm{~s}^{-1}$ as of August 2008. One can also speak of the integrated

luminosity, $\int_{t_{0}}^{t} \mathcal{L} d t$, which is a measure of the total luminosity over a period of time. DØ has recorded over $4 \mathrm{fb}^{-1}\left(1 \mathrm{fb}=10^{-15}\right.$ barns, 1 barn $\left.=10^{-24} \mathrm{~cm}^{2}\right)$ of integrated luminosity during RunII as of August 2008.

\subsection{The DØ Detector}

The $\mathrm{D} \varnothing$ detector is one of two detectors located on the Tevatron. It is a general-purpose detector consisting of three major subsystems: a central 


\begin{tabular}{|c|c|c|c|}
\hline Parameter & RunI & RunIIa & RunIIb \\
\hline Beam Energy & 900 & 980 & 980 \\
Bunches & 6 & 36 & 36 \\
Protons/Bunch & $2.3 \times 10^{11}$ & $2.7 \times 10^{11}$ & $3 \times 10^{11}$ \\
Anti-protons/Bunch & $5.5 \times 10^{10}$ & $3.0 \times 10^{10}$ & $7 \times 10^{10}$ \\
Bunch spacing (ns) & 3500 & 396 & 396 \\
Peak Inst. Luminosity (cm $\left.{ }^{-2} \mathrm{~s}^{-1}\right)$ & $1.6 \times 10^{31}$ & $1.5 \times 10^{32}$ & $3 \times 10^{32}$ \\
$\int \mathcal{L} d t\left(\mathrm{pb}^{-1} /\right.$ week $)$ & 3.2 & 17.3 & 50 \\
\hline
\end{tabular}

Table 2.1: Tevatron Operating parameters for RunI, RunIIa, and RunIIb.

tracker surrounded by a solenoid magnet; a hermetic liquid-argon (LAr) calorimeter; and a muon spectrometer with a toroidal magnet. The detector has been in operation since 1992 and recorded $\approx 125 \mathrm{pb}^{-1}$ during Run I, and has recorded over $4 \mathrm{fb}^{-1}$ during RunII as of August 2008. Figure 2.2 shows a side view of the entire detector and outlines the major subsystems. Full details of the detector can be found in Refs [21],[22], and [23]. Below we discuss the detector subsystems in more detail.

\subsubsection{The DØ Coordinate System}

$\mathrm{D} \varnothing$ uses a right-handed coordinate system with the $+z$ axis in the direction of the proton beam (south). In Cartesian coordinates the $+x$ axis points east and the $+y$ axis is normal to the earth. DØ most often uses cylindrical or spherical coordinates; in cylindrical coordinates the azimuthal angle $\phi$ lies in the plane transverse to the $z$-axis (the "transverse plane") and is measured from the $+x$ axis. In spherical coordinates the azimuthal angle is the same, and the polar angle $\theta$ is measured from the $+z$-axis. Most often, however, the polar angle is replaced with the pseudorapidity, denoted as $\eta$, defined as

$$
\eta \equiv-\ln \left(\tan \left(\frac{\theta}{2}\right)\right)
$$

The pseudorapidity is equivalent to the rapidity $y$ when a particle's mass is negligible. $y$ is defined as

$$
y=\frac{1}{2} \ln \left(\frac{E+p_{z}}{E-p_{Z}}\right)
$$




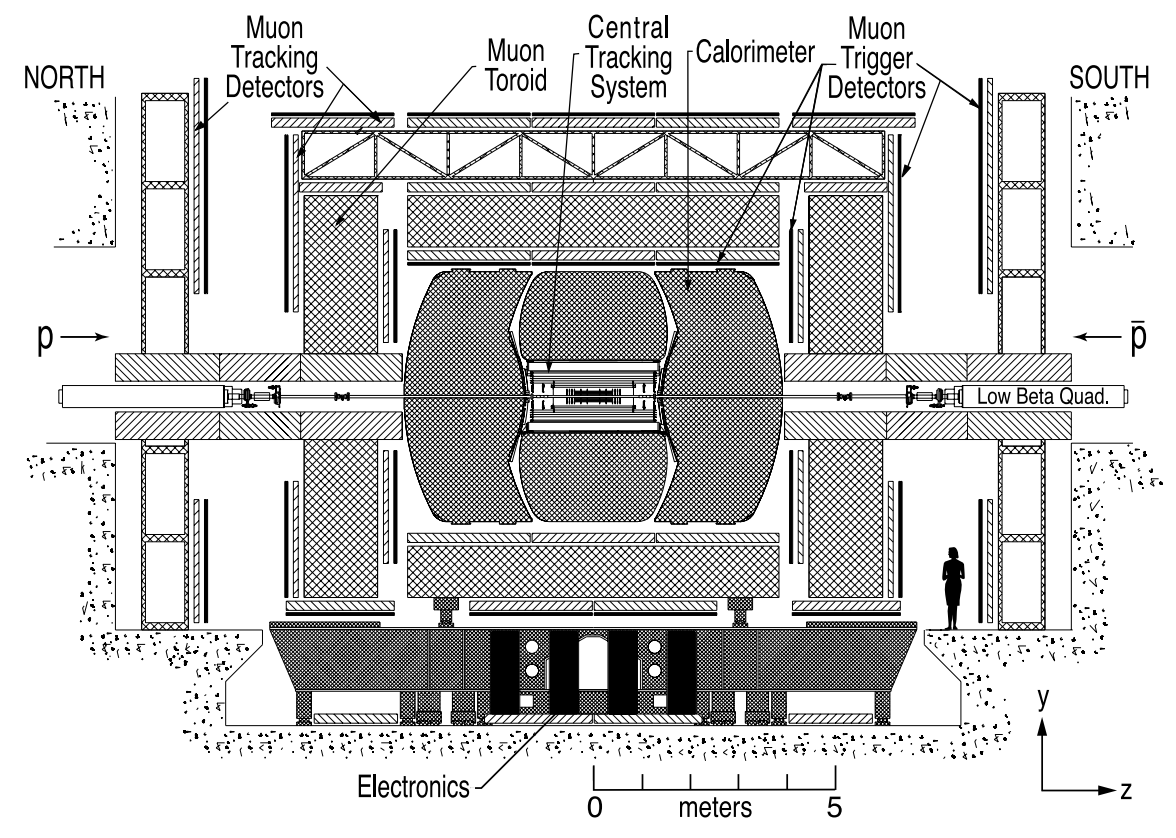

Figure 2.2: Side view of the DØ detector with major subsystems labeled [22].

\subsubsection{Silicon Microstrip Tracker}

The innermost subdetector is the Silicon Microstrip Tracker (SMT). It consists of six barrel detectors, with twelve F-disk detectors interspersed between the barrels, with four (inner and outer on both sides) H-disk detectors on the outside (farthest in $z$ from the detector center.) The detectors have $\approx 15 \mu \mathrm{m}$ position resolution and are capable of 3-D track reconstruction. Figure 2.3 shows the SMT layout. Counting all barrel, F-disk, and H-disk sensors, the SMT has approximately 800,000 readout channels.

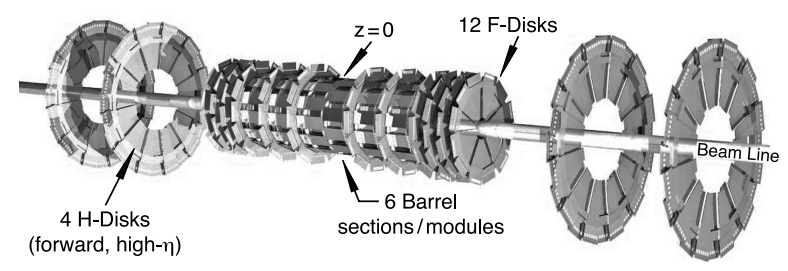

Figure 2.3: SMT layout [22]. The two outer H-disks were removed during the 2006 RunIIb upgrade.

The six barrel detectors are $12 \mathrm{~cm}$ in length along the $z$ axis and are 
centered at $|z|=6.2,19.0,31.8 \mathrm{~cm}$. During RunIIa the barrels consisted of four concentric silicon sensor layers at radii of $2.715,4.55,7.582$, and $10.51 \mathrm{~cm}$. During spring 2006, an additional layer (called Layer 0) for the silicon tracker was installed close to the beam pipe, improving charged particle momentum resolution and heavy flavor identification. Figure 2.4 shows a cross-section of the SMT module design (Layer 0 is not shown.) As the barrel detectors lie in the central $\eta$ region they primarily measure the $r-\phi$ position of tracks. The barrel silicon sensors are a mix of Single-sided (SS), Double-sided (DS), and Double-sided Double-metal (DSDM) sensors. The four central barrels use DSDM sensors for layers 1 and 3, and DS sensors for layers 2 and 4 . The outer two barrels use SS for layers 1 and 3 and DS for layers 2 and 4 . The DS sensors have a $15^{\circ}$ stereo offset between the two sides, while the DSDM sensors have a $90^{\circ}$ offset.

The F-disk detectors lie at $|z|=12.5,25.3,38.2,43.1,48.1$, and $53.1 \mathrm{~cm}$. The F-disks contain trapezoidal sensors in a wedge arrangement. They use double-sided sensors with the strips of one layer having a $30^{\circ}$ stereo offset to the other. The inner radius of the F-disks is $2.57 \mathrm{~cm}$, while the outer radius is $9.96 \mathrm{~cm}$.

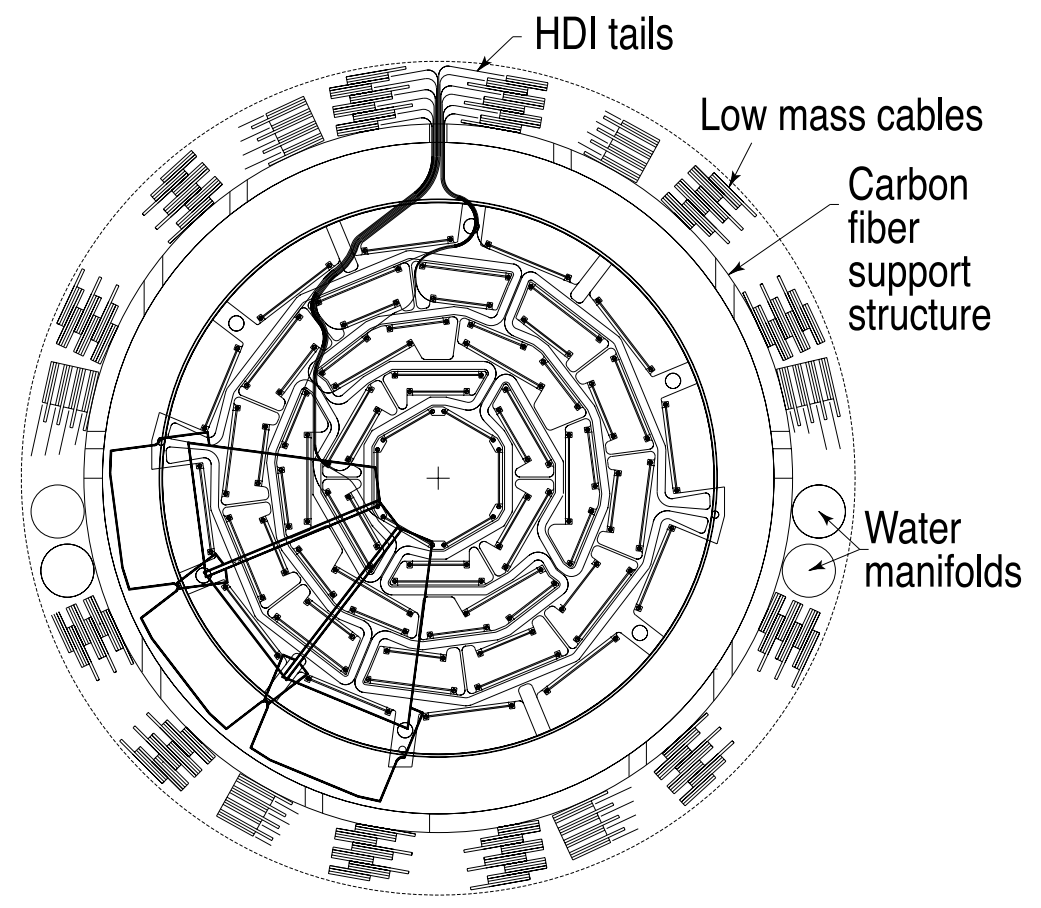

Figure 2.4: SMT disk/barrel module cross section [22]. Layer 0, added in 2006, is not shown. 
The H-disk detectors provide forward coverage up to $|\eta| \approx 3$. The H-disk centers lie at $|z|=100.4,121.0 \mathrm{~cm}$. During the RunIIb upgrade in 2006, the two outer H-disks were removed to make room for Layer 0 electronics and support modules. The H-disks consist of back-to-back single-sided sensors in a half-wedge shape, providing an effective $15^{\circ}$ stereo angle between the two sides. The inner radius of the H-disks is $9.5 \mathrm{~cm}$ and outer radius is $26 \mathrm{~cm}$.

All disks and barrels use SVXIIe chips for readout in the outer four layers, while SVX4 chips are in Layer 0. The SVX chips read out through a High Density Interconnect (HDI), mounted on a Kapton flex circuit. The SVX chips generate a significant amount of heat during operation, and must be kept cold. Cooling comes in the form of an ethylene-glycol mixture, kept at $-10^{\circ} \mathrm{C}$, passed through the SMT support structures as shown in figure 2.4. The detector area is also purged with dry air having a typical dewpoint of $-40^{\circ} \mathrm{C}$.

\subsubsection{Central Fiber Tracker}

The Central Fiber Tracker (CFT) surrounds the SMT and consists of scintillating fibers connected to visible light photon counters (VLPCs) arranged in eight concentric layers, labeled A through $\mathrm{H}$ in order of increasing radius. The inner two layers have a length of $1.66 \mathrm{~m}$ in $z$; they are shorter than the remaining six layers (length $2.52 \mathrm{~m}$ ) to accommodate the SMT H-disks. The CFT provides tracking coverage up to $|\eta|<1.6$ in the A and B layers and $|\eta|<1.8$ in the outer six layers. Figure 2.5 shows a CFT side view. The CFT layers range in radius from $20 \mathrm{~cm}$ to $52 \mathrm{~cm}$. Each of the eight layers consists of an axial (fibers aligned along the beam direction) and stereo (fibers are offset by $\pm 3^{\circ}$ from the axial fibers) sublayers to provide additional position information. The fiber diameter is $835 \mu \mathrm{m}$. The CFT's inherent position resolution is about $100 \mu \mathrm{m}$ due to the double layer structure. Each sublayer has from 1,280 fibers (in the A layer) to 3,520 fibers (in the $\mathrm{H}$ layer.)

As a charged particle passes through the CFT, it will cause the fibers to scintillate. This light then passes through waveguides to the VLPCs, which convert the light to an electronic signal. The VLPCs operate at a temperature of about $9 \mathrm{~K}$ and have a quantum efficiency of roughly $75 \%$. There are $\approx 77,000$ VLPC readout channels in the CFT. All three levels of the trigger system (sections 3.1.1, 3.1.2, 3.1.3) use the CFT information for track reconstruction. 


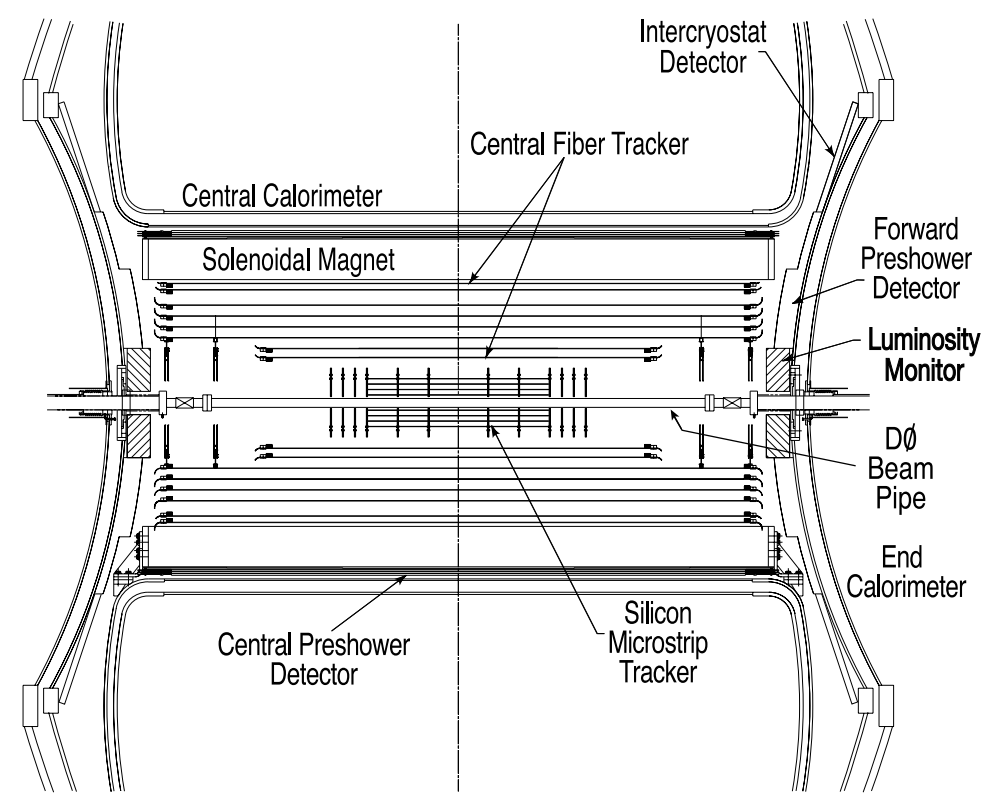

Figure 2.5: Side view of the D $\varnothing$ central tracker showing the SMT and CFT[22].

\subsubsection{Solenoid and Toroid}

The solenoid magnet encloses the central tracking system (SMT and CFT). It is $2.73 \mathrm{~m}$ long in $z$, has an inner (outer) diameter of $1.07(1.42) \mathrm{m}$ and is 0.87 radiation lengths $\left(X_{0}\right)$ thick.. It is made from a superconducting $\mathrm{Cu}: \mathrm{NbTi}$ material and produces a field of $1.92 \mathrm{~T}$ while drawing 4,750 A of current. It is designed to enable the tracker's momentum measurement by curving charged particles in the tracking detector, and resides in its own cryostat cooled with liquid helium. The magnetic field will cause extremely low momentum particles to circle with too small a radius to reach the tracker; this results in an effective lower $p_{T}$ limit on track reconstruction of $500 \mathrm{MeV}$.

The toroid magnets lie between the A and B layers in the muon system. There is a central toroid and one on each end in the forward muon system. The central toroid has a magnetic field of $1.8 \mathrm{~T}$ and the end toroids' fields are $1.9 \mathrm{~T}$, drawing 1,500 A of current. The toroids provide curvature in the muon system and enable a standalone $p_{T}$ measurement in the muon system, allowing for a low- $p_{T}$ cutoff in the Level1 muon trigger, improved matching to tracks from the central tracker, $\pi / K$ rejection, and improved resolution at high $p_{T}$. 


\subsubsection{Central and Forward Preshower Detectors}

The preshower detectors consist of interleaved triangular strips of plastic scintillator, inside of which are wavelength-shifting fibers (WLS), shown in figure 2.6. The central preshower detector (CPS) is located between the solenoid magnet and the calorimeter. It consists of three layers arranged in an axialstereo-stereo manner, with the inner layer being axial and the outer two layers having stereo angles of $\approx \pm 24^{\circ}$. A layer of lead and stainless steel radiator approximately one radiation length thick is mounted on the solenoid and in front of the preshower detectors to aid in the discrimination of electrons and photons. The WLS fibers are split at $z=0$ and then read out from both ends by VLPCs.

The forward preshower detectors are mounted on the calorimeter endcaps. They consist of two layers of trapezoidal modules arranged in a wedge with a lead-stainless steel absorber, approximately two radiation lengths thick, in between them. Both layers have two sublayers of strips offset by a stereo angle of $22.5^{\circ}$ and covering $22.5^{\circ}$ of azimuthal angle. The layers closest to interaction region (i.e. in front of the absorber) are known as MIP (minimum ionizing particle) layers, while those behind the absorber are known as shower layers.

As a particle travels through the preshower detectors it will cause the scintillating plastic to emit blue light. The wavelength shifting fiber shifts the light into green, and the fibers are read out by the same type of VLPC as is in the CFT. The preshower detectors aid in discriminating electrons and photons through the interaction with the absorber. A charged particle like an electron will act as a MIP in the MIP layers, leaving behind a cluster of energy in both the MIP and shower layers. Photons, however, will not leave a deposit in the MIP layer, but will show energy only in the shower layers after passing through the absorber.

\subsubsection{Calorimeter}

The calorimeter, shown in figure 2.7, is responsible for measuring the energy, direction, and shower shape of electrons, hadronic jets, and photons. A partially-compensating sampling calorimeter, the $\mathrm{D} \varnothing$ calorimeter consists of a central calorimeter (CC), providing coverage in the $|\eta|<1.1$ region, and two endcap calorimeters on the north and south ends of the detector (ECN, ECS) that provide coverage from $|\eta|>1.3$ to $|\eta|<4.2$. All three are housed in separate cryostats. Two intercryostat detectors, discussed below, provide coverage in the cryostat gaps.

The calorimeters are each divided into three main sections. From closest to farthest from the interaction point they are the electromagnetic (EM), fine 


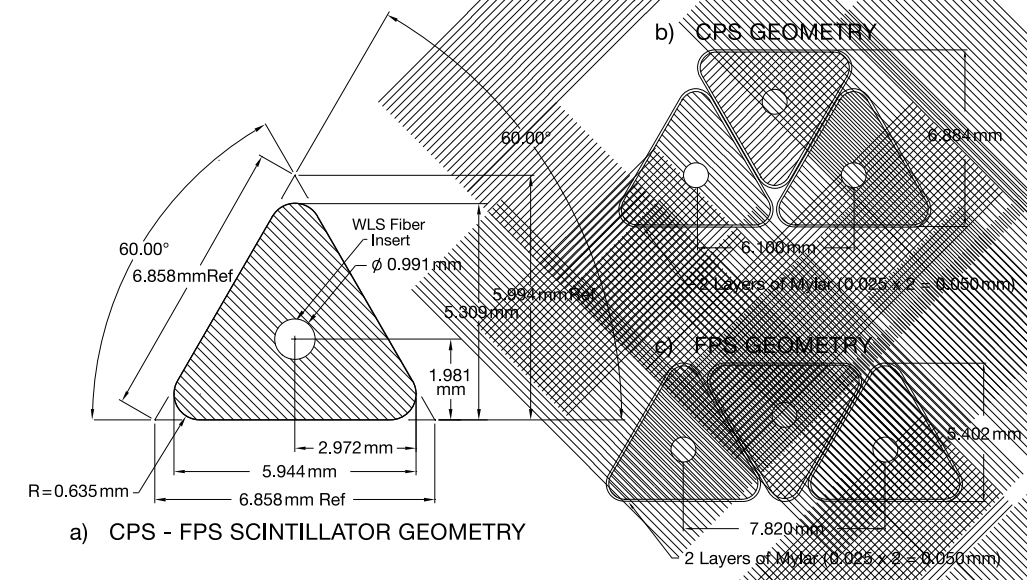

Figure 2.6: Geometry of the preshower scintillator strips. The circles show the of the wavelength-shifting fibers (WLS) [22].

hadronic $(\mathrm{FH})$, and coarse hadronic $(\mathrm{CH})$. There are four sublayers in the EM section, three in the fine hadronic sections, and one in the coarse hadronic section. Each is composed of many unit cells, combined in "towers" approximately $0.1 \times 0.1$ in $\eta-\phi$ space, as shown in figure 2.8. In the third EM sublayer the segmentation is twice as fine so as to give better resolution near the EM shower maximum, and tower size increases to $0.2 \times 0.2$ for $|\eta|>3.2$. The cells use liquid argon (LAr) as an active ionization medium, and either uranium, copper, or stainless steel as an absorber. Figure 2.9 shows the design of a calorimeter cell.

As particles pass through the calorimeter they will lose energy, shower, and ionize the liquid argon (or, in the case of neutral particles such as neutrons, produce fission in the uranium which will create extra ionizing particles.) The ions will drift to the readout plates, kept at a $2 \mathrm{kV}$ potential, and the amount of charged collected is proportional to the particles' energy. The readout plates are made with G-10 except in the small-angle EM region in the ECN and ECS, where they are printed circuit boards. Since the drift time for the ions in the LAr is about $450 \mathrm{~ns}$, but the time between interactions is only $396 \mathrm{~ns}$, it is possible for there to be ions from different interactions in the calorimeter at once; this is known as pileup. Thus a Base Line Subtraction (BLS) board is part of the readout system and it subtracts the signal from 396 ns ago from the current signal to remove the previous events.

As mentioned there is a gap in $\eta$ coverage in the space between the three cryostats. The Inter-Cryostat Detector (ICD) is a subdetector made of 0.5inch thick scintillating tiles mounted on the end cryostats. It covers the region 
$1.1<|\eta|<1.4$. Each tile provides approximately $\Delta \eta \times \Delta \phi=0.3 \times 0.4$; each tile is divided into 12 subtiles to give $\Delta \eta \times \Delta \phi=0.1 \times 0.1$ resolution. Each subtile contains wavelength-shifting fibers read out by photomultiplier tubes. Additionally, from $0.8<|\eta|<1.4$ there are some locations with unsampled material in the calorimeter. Here, single-cell readout structures called massless gaps have been placed in front of the first layer of uranium, using the cryostat as the absorber.

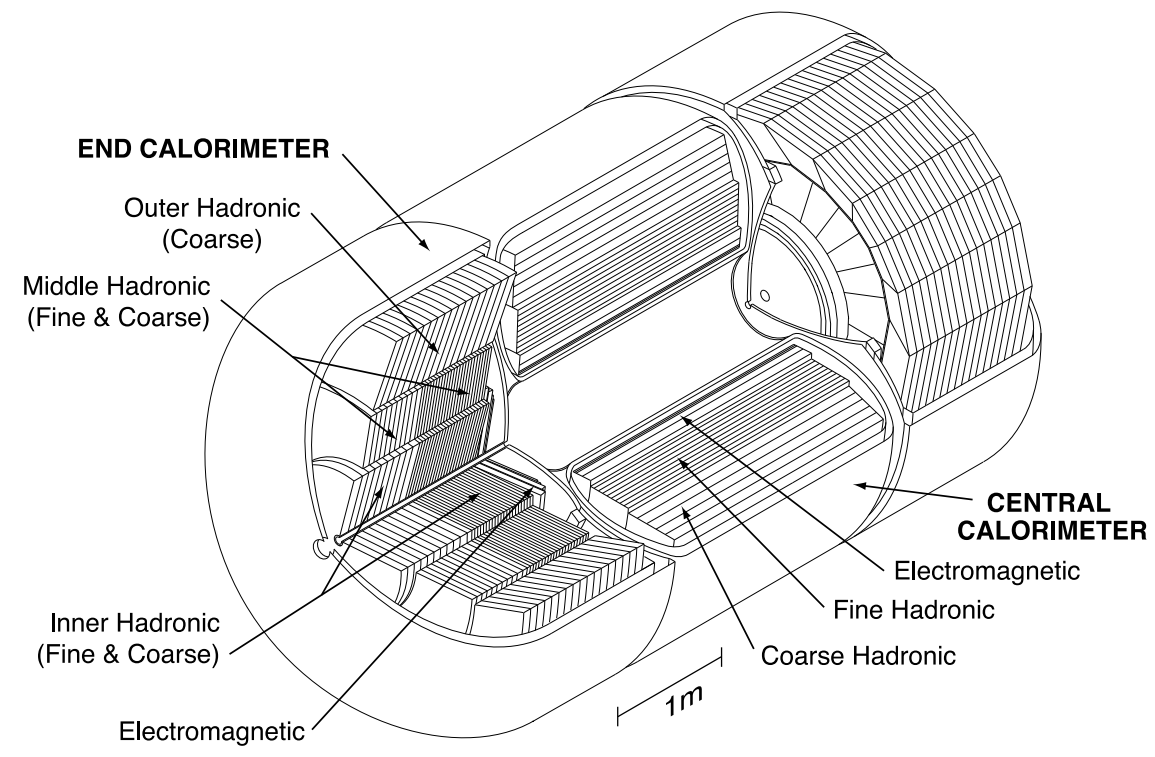

Figure 2.7: The DØ calorimeter[22].

\subsubsection{Muon System}

The outermost section of the detector is the muon system. It consists of a central and forward system, providing coverage from $|\eta|<1.0$ and $1.0<|\eta|<$ 2.0 , respectively. Figure 2.10 shows an exploded view of the muon system.

The central system consists of a toroidal magnet (sec. 2.2.4), three layers of drift chambers, a cosmic cap and the $A \phi$ scintillation counters. Due to the support structure needed for the entire detector, the bottom $(4.25<\phi<5.15$ of the central muon system is not fully instrumented. Muon reconstruction is possible but the efficiency is much lower. The three layers of drift chambers are known as the $\mathrm{A}, \mathrm{B}$, and $\mathrm{C}$ layers in order of increasing distance from the interaction point. The toroidal magnet is between the A and B layers. The drift chambers consist of Proportional Drift Tubes (PDTs) with an anode wire 


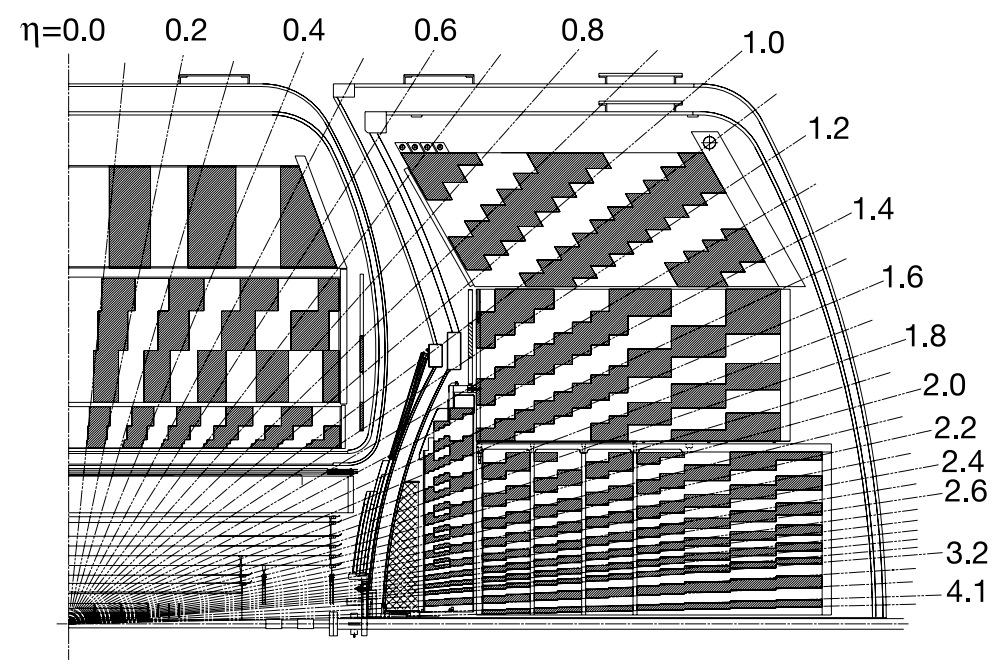

Figure 2.8: Cross section of the $\mathrm{D} \varnothing$ calorimeter showing tower and cell segmentation. The shading patterns show cells that are grouped together into a tower during signal readout[22].

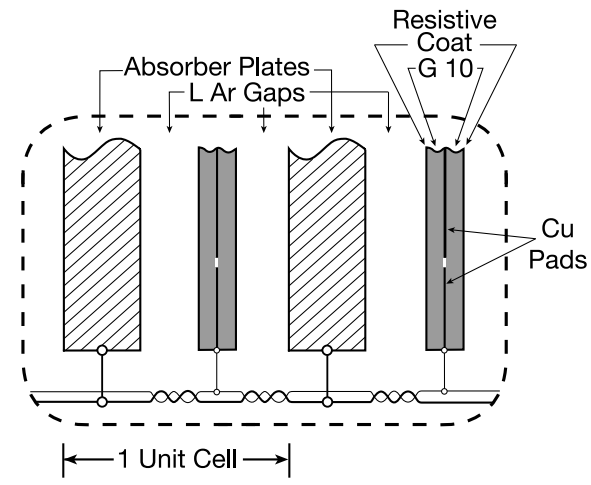

Figure 2.9: Design of a typical DØ calorimeter cell[22]. 
and cathode pads to provide position information along the wire. Adjacent wires are paired together for $\Delta T$ measurements during readout. As a muon passes through a PDT, the PDT records the $\Delta T$ between hits on the wire pairs as well as the charge deposition; both quantities go into the hit position calculation. Outside of the drift chambers are the cosmic caps; made of Bicron scintillator and designed to veto against muons coming from cosmic rays. The $A \phi$ scintillation counters surround the A-layers PDTs, a shown in figure 2.11, and have several functions: they provide a time measurement for low- $p_{T}$ muons that do not penetrate the toroid (and thus do not reach the B and C layers), the provide a means to reject out-of-time back-scattering from the forward region, and provide fast hit information to match to tracks from the CFT for use in muon triggers at Level1.

The forward muon system consists of the end toroids (2.2.4), mini-drift tubes (MDTs), scintillation counters for triggering, and beam pipe shielding. The MDTs are arranged in three layers, also called A, B, and C, and are filled with a $\mathrm{CF}_{4}-\mathrm{CH}_{4}$ gas mixture. There is an anode wire connected to an amplifier and discriminator board; from there the signal arrival time is compared to the beam crossing time. The MDT efficiency in the active area is nearly $100 \%$ and the intrinsic position resolution is $\approx 0.7 \mathrm{~mm}$. The Trigger Scintillation Counters are in each of the three layers and they are divided into octants as shown by the 3 -axis in figure 2.11. They are segmented in $4.5^{\circ}$ in $\phi$ to match the 80 CFT sectors, and at $0.12(0.07)$ in $\eta$ in the first nine(last three) of the 12 rows within each layer. Their function is to provide timing information for the Level 1 muon trigger 3.1.1. The beam shielding consists of iron, polyethylene, and steel near the beam pipe and low- $\beta$ quadrupole magnets to prevent any proton or antiproton remnants from reaching the muon system, and to shield the muon system from beam halo effects.

All three trigger levels use muon information in trigger decisions. 


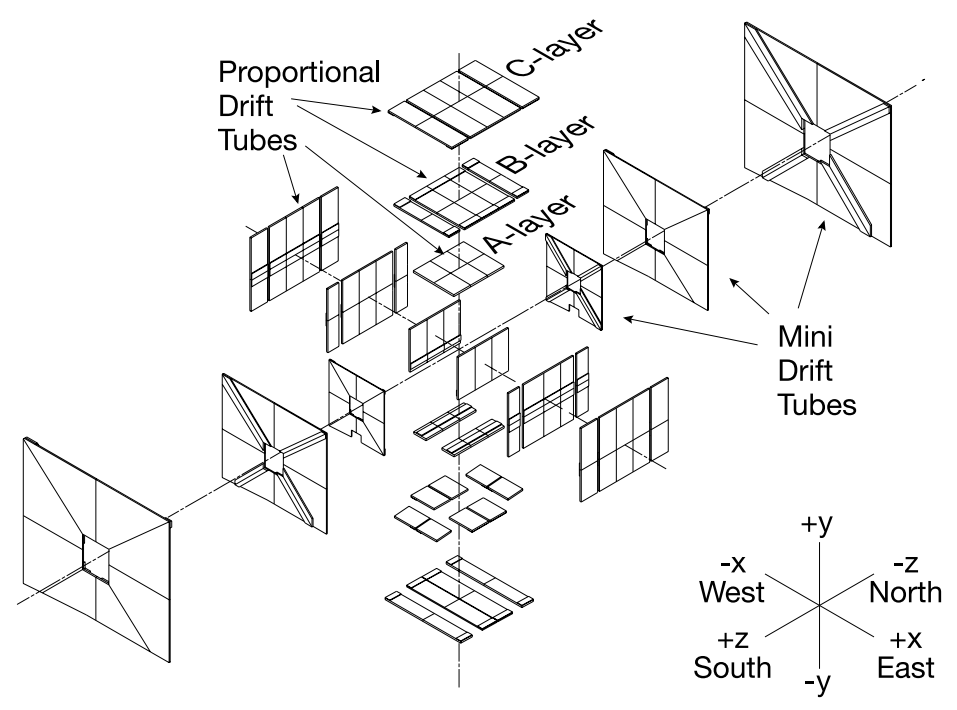

Figure 2.10: An exploded view of the muon system, the outermost part of the $\mathrm{D} \varnothing$ detector. Only the wire, i.e. drift chamber, portion is shown here.[22].

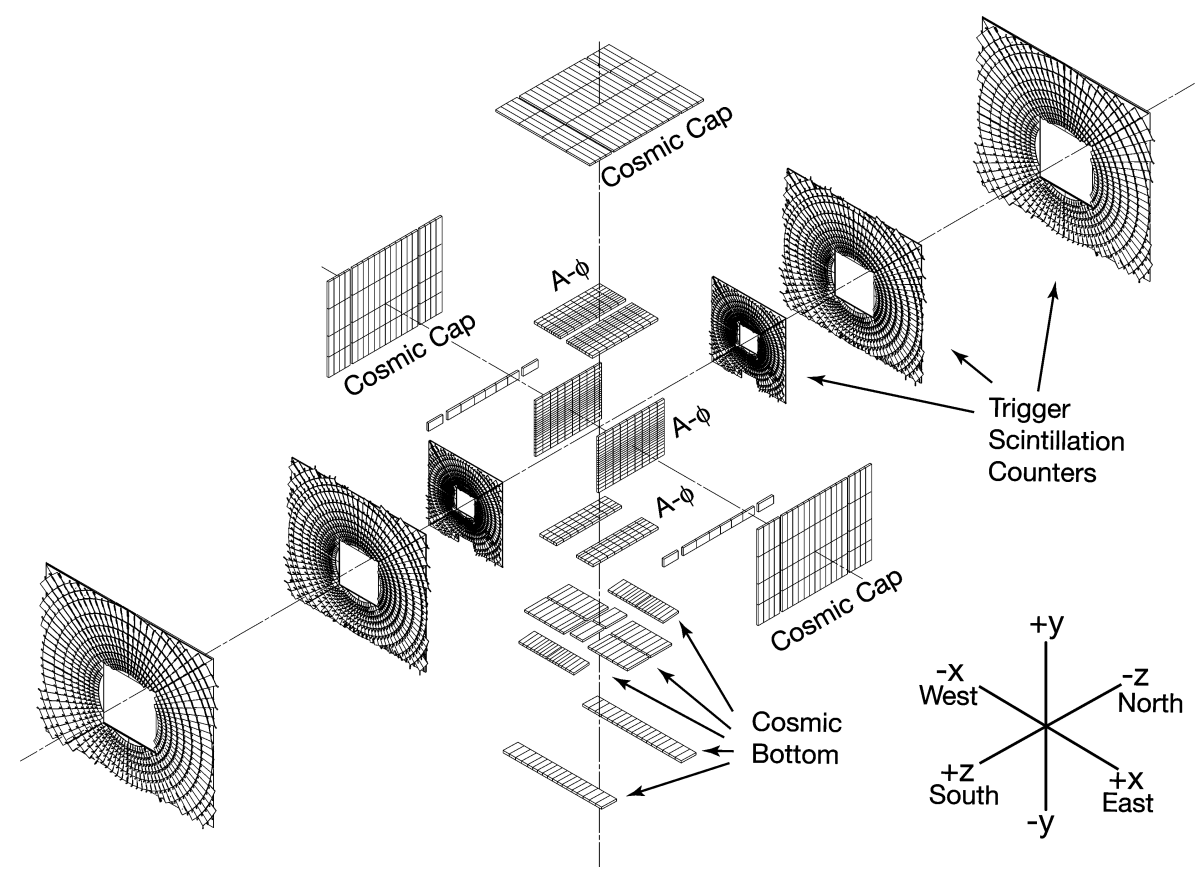

Figure 2.11: An exploded view of the muon system, with the scintillator systems ( $A \phi$ scintillators and cosmic caps in the central system, trigger scintillation counters in the forward system) shown [22]. 


\section{Chapter 3}

\section{Trigger and Data Acquisition}

\subsection{The DØ Trigger System}

Collisions occur within the $\mathrm{D} \varnothing$ interaction region at a rate of approximately 2.5 $\mathrm{MHz}$. It is impossible to record every event, most of which are low-energy QCD processes not of interest to the research program. Thus a robust and efficient system is needed to pick out events containing interesting interactions. This system is called a trigger system, and presently $\mathrm{D} \varnothing$ has a three-level trigger system, designed to pick out high- $p_{T}$ particles such as muons, electrons, taus, and jets, to identify events of interest to all physics programs. Figure 3.1 shows an overview of the trigger system and its major components.

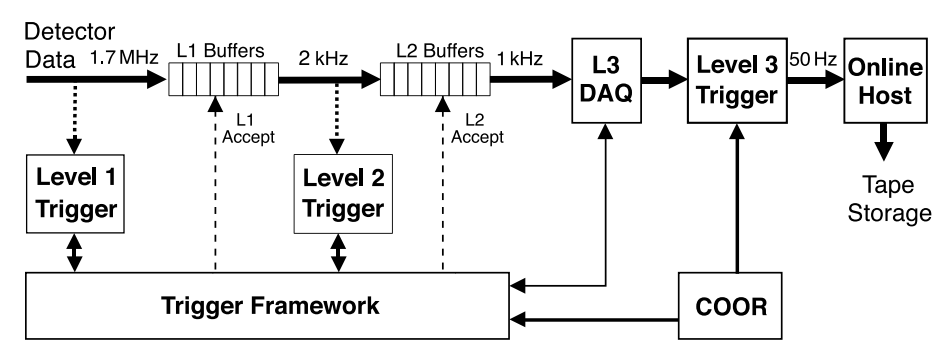

Figure 3.1: Overview of the D $\varnothing$ trigger system [22].

\subsubsection{Level 1 Trigger}

The Level 1 trigger system is designed to take the $2.5 \mathrm{MHz}$ collision rate and retain events of interest with an output event rate of between 1 and 2 kHz. It allows up to 128 distinct trigger terms. Figure 3.2 shows a schematic 
of the Level 1 trigger system. The major subsystems include the Level 1 Central Track Trigger, or L1CTT, the Level Muon Trigger (L1Muon), Level 1 Calorimeter Trigger (L1Cal), and Level 1 CalTrack. The Trigger Framework controls and synchronizes each subsystem.

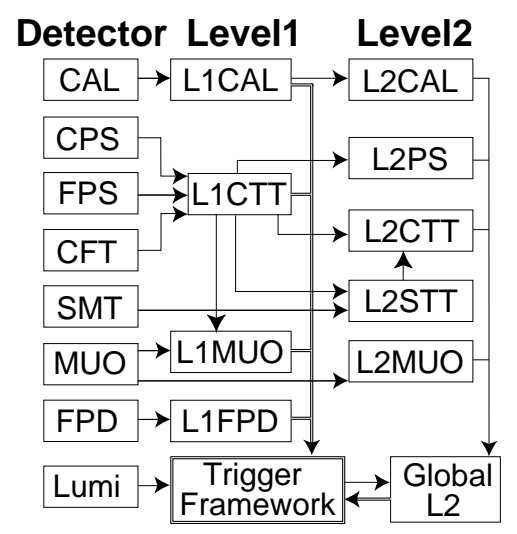

Figure 3.2: Diagram of the Level 1 and Level 2 trigger system [22].

\section{Level 1 Central Track Trigger}

The Level 1 Central Track Trigger (L1CTT) [24] takes readout from the CFT and the preshower detectors to reconstruct $(r, \phi)$ trajectories of track originating near the beam line. Events can be selected on track $p_{T}$, and/or isolation. The outputs from the CFT AFE boards go to digital front end (DFE) boards where the CFT hits are compared with $\approx 20,000$ predefined tracks. There are four different $p_{T}$ bins for L1 tracks, two $p_{T}$ bins for isolated tracks, and the tracks can be matched to preshower clusters for electron triggering. Output tracks from L1CTT go to L1 Muon for track-matched muon triggering, L1CalTrack, L2STT, L2CFT, and Level 3 after a level 1 accept.

\section{Level 1 Muon Trigger}

The Level 1 Muon trigger (L1Muon) [22, 23] trigger receives input from L1CTT and directly from the muon hardware. It creates trigger terms based on the wire and scintillator hit and timing information (loose, medium, and tight timing settings for both the scintillator and wire requirements) and $p_{T}$ of a matched L1CTT track if required. Additionally several $\eta$ cuts can be made, corresponding to the entire muon system coverage $(|\eta|<2.0)$, the region covered by the CTT $(|\eta|<1.6)$, the central muon system only $(|\eta|<1.0)$, or the forward muon system only $(1.0<|\eta|<2.0)$. 


\section{Level 1 Cal Trigger}

The Level 1 Calorimeter Trigger (L1Cal) takes input from the calorimeter front ends to form calorimeter objects such as jets, taus, electrons, photons, and missing transverse energy $\left(E_{T}\right)$ for use in fast triggers. Details of the design for RunIIa are in [25]. During the RunIIb upgrade in 2006, D $\varnothing$ completely redesigned the system to be able to cope with the increase in instantaneous luminosity, while maintaining and in some cases improving selection efficiency for interesting physics processes. Full details of the current design are in $[26,27]$. To identify jets, photons, taus, and electrons L1Cal uses variants of the Sliding Windows algorithm [27, 28]. The algorithms do parallel searches of adjacent calorimeter towers in order to find local maxima of transverse energy $\left(E_{T}\right)$ deposition. For jets, this involves summing several trigger towers (a trigger tower is $0.2 \times 0.2$ in $\Delta \eta \times \Delta \phi$ space), into trigger tower clusters (TTCLs) and summing the $E_{T}$ in the surrounding $4 \times 4$ TT space, or $0.2 \times 0.2$ in $\Delta \eta \times \Delta \phi$ space. For electrons, the TTCLs are only $1 \times 1$, and the $E_{T}$ sum is over the local maximum and one neighboring tower with the next highest $E_{T}$. This is because electrons have a very narrow shower. Additionally one can measure the EM/HD (hadronic) fraction and apply isolation cuts on the EM candidates, as electrons are unlikely to deposit much energy in the hadronic calorimeter and will have little energy in surrounding towers. The tau algorithms are very similar to the jet algorithms, but they also compute the $E_{T}$ ratio in the $2 \times 2$ region over the $4 \times 4$ region to exploit the fact that taus have a narrower shower than hadronic jets. Additionally, one can create topological or missing $E_{T}$ trigger terms looking at all jet or EM objects in the event.

\section{Level 1 CalTrack}

Another new system in RunIIb is Level 1 CalTrack [29]. This system allows matching of L1CTT tracks to jets and electrons from L1Cal, resulting in substantially lower trigger rates, while preserving objects of interest. L1CTT tracks with transverse momenta that satisfy preset $p_{T}$ thresholds are matched in $\phi$ to jets or electrons matching $E_{T}$ and $\eta$ requirements. Additionally, track isolation and preshower confirmation for electrons and jets is also possible. Trigger terms are constructed out of the various track $p_{T}$, jet/electron $E_{T}$, $\eta$, preshower, and isolation possibilities. The muon+tau triggers used in this analysis make extensive use of these trigger terms. 


\subsubsection{Level 2 Trigger}

The Level 2 trigger is a combination of hardware and software; it is designed to reduce to the trigger rate to $500-1000 \mathrm{~Hz}$. It consists of subdetector-specific preprocessors, which are hardware boards that receive input from the detector front ends and/or from the L1 trigger system, and a Level 2 Global processor, which compares objects across the entire event and makes the final decision at Level 2. The detector-specific preprocessors run in parallel and can create more detailed and higher-quality physics objects than the Level 1 systems. Figure 3.2 shows a diagram of the level 2 trigger system, and its connection to the trigger system as whole.

\section{L2 Muon Preprocessor}

The L2 Muon system receives input from L1 Muon and the PDT, MDT, and scintillation counter front end modules. It uses eighty digital signal processors arranged in groups of five (called Second Level Input Computers, or SLICs) to compute in parallel track segments for each portion of the detector. Level 2 muons contain track $p_{T}, \eta, \phi$, quality, and scintillator timing information with a higher quality than level 1 muons.

\section{L2 CTT Preprocessor}

The L2CTT processor receives its input from L1CTT and from L2STT. It can either accept tracks directly from L1CTT, or from L2STT (sec. 3.1.2, which uses L1CTT tracks as seeds and refines them with SMT barrel detector hits. It outputs two track lists to L2 global and to L3 for bookkeeping: the first list is $p_{T}$-sorted and the second is impact parameter (IP)-sorted. Triggers use both lists; the list choice is driven by the physics process of interest.

\section{L2 Cal Preprocessor}

The L2 Calorimeter preprocessor is capable of identifying jets, taus, electrons, and photons, as well as determining $\mathbb{E}_{T}$. It takes the trigger towers as input. For jets, the algorithm computes $E_{T}$ sums over $5 \times 5$ towers clusters, centered on a seed tower. Overlapping candidates may in some cases be identified as multiple jets. The tower clusters are sent to the L2 Global processor (sec. 3.1.2) which applies the jet requirements in the trigger menu. For electrons, photons, and taus, seed towers and the neighboring tower with the highest $E_{T}$ (seed + "nearest neighbor") make up the core of the object, with the $E_{T}$ sum in the $3 \times 3$ tower region (centered on the seed tower) a variable

available for use in trigger design. A cut on the $\frac{E_{T(s e e d+N N)}}{E_{T(3 \times 3)}}$ ratio can be made 
on tau candidates, similar to the ratio cut at L1; this is useful for background rejection as true hadronic taus have a higher ratio. We will discuss the tau trigger requirements on the triggers used for this analysis in 3.2.2. The $\mathbb{E}_{T}$ algorithm simply calculates the vector $E_{T}$ sum over all trigger towers passed from L1 that meet the chosen $\eta$ and individual tower $E_{T}$ sum requirements.

\section{L2 PS Preprocessor}

The Level 2 Preshower receives the CPS axial and stereo clusters (i.e. groups of hits) upon a L1 accept. It computes cluster centroids and looks for $\eta$ and $\phi$ matches in three layers. Cluster matches that also have a matching CFT track are considered electrons; those without, photons. The $\eta$ and $\phi$ coordinates are binned to match the $\Delta \eta \times \Delta \phi=0.2 \times 0.2$ calorimeter trigger towers, with preshower hits being considered calorimeter matches if they lie within a window of size 0.05 around a trigger tower. The FPS clusters are treated in the same way, but processed independently.

\section{L2STT Preprocessor}

The Silicon Track Trigger (L2STT or STT) [30] is a dedicated preprocessor that takes input from L1CTT and the raw SMT data streams in order to form tracks from the SMT hits, using L1CTT tracks as seeds. There must be hits in at least four of the five SMT layers to form an STT track. The STT is designed to search for tracks with large impact parameter; tracks from a $b$ quark decay often have high impact parameters. To that end the STT also corrects for the beam position to give a more accurate estimate of a track's impact parameter. The STT sends its output to the L2CTT processor to be used in the trigger decision. On a L2 accept the STT also sends its output to level 3 for bookkeeping. A more detailed treatment of the STT is available in Appendix A.

\section{Level 2 Global Processor}

The Level 2 Global processor takes input from all L2 preprocessors as well as the L1 trigger decisions from the Trigger Framework. For each event, the processor examines the level 1 trigger bits to see what L1 trigger fired, and then only runs the L2 algorithms associated with L1 scripts that fired. A trigger can contain any type of L2 object or objects, and it can also match objects of different types together in $\eta$ and/or $\phi$ space. For example, a typical muon+hadronic tau trigger will require a L2 Tau (from the L2 Cal preprocessor) to be matched with an STT track (from L2STT). Many other combinations are of course pos- 
sible. If any L2 trigger passes, L2 global will issue an accept to the Trigger Framework and the event moves to Level 3.

\subsubsection{Level 3 Trigger}

The Level 3 trigger [31] is the final trigger level at DØ; it applies the most precise software algorithms to the digitized detector and L2 outputs and applies selection requirements. The output rate is $50-100 \mathrm{~Hz}$. The algorithms run on a cluster of roughly $400 \mathrm{PCs}$ known as the Level 3 farm, with events processed in parallel across the farm. There are specific algorithms for the full range of physics objects, including muons, taus, jets, electrons, tracks, $\mathbb{E}_{T}, b$-jets, and various topological combinations, the detail of which is roughly comparable to basic offline reconstruction algorithms, but with somewhat less precision due to speed constraints. The L3 trigger performs exceptionally well, even at the highest instantaneous luminosities and add essentially no latency to the overall event processing time. We will discuss the L3 algorithms important to this analysis in more detail in sections 3.2.1 and 3.2.2.

\subsection{Trigger Design and Implementation}

$\mathrm{D} \emptyset$ has triggers optimized for nearly every area of its physics program. Some triggers are solely object based, i.e. $n$ objects of a certain type of types at each trigger level, while others are topology based, i.e. some objects with some angular separation, etc. In this analysis we require a data event to fire at least one of nine dedicated high- $p_{T}$ single muon triggers (hereafter called single muon triggers), or one of twenty-four muon +hadronic tau triggers. Below we describe the structure and requirements of these triggers at each level, how they work within the trigger system, and how to calculate the efficiency of the trigger requirement as whole so it can be applied to simulated events.

\subsubsection{Single Muon Triggers}

Previous analyses in this channel used single muon triggers exclusively. In the time period spanned by this analysis, single muon triggers had three different level 1 (L1) and level 2 (L2) requirements, and three different level 3 (L3) requirements, to form a total of nine triggers.

The three possibilities for the L1/L2 requirements with are as follows:

- MUHI1- L1: A muon in the wide region $(|\eta|<1.6)$, tight scintillator timing and loose wire requirements, matched to a CTT track with $p_{T}>13$ 
GeV. L2: A medium-quality muon with $p_{T}>3 \mathrm{GeV}$ and tight scintillator timing cuts, OR an STT track with $p_{T}>20 \mathrm{GeV}$.

- MUHI2- L1: A muon in the wide region $(|\eta|<1.6)$, tight scintillator timing and tight wire requirements, matched to a CTT track with $p_{T}>8$ $\mathrm{GeV}$. There must also be an isolated L1CTT track with $p_{T}>10 \mathrm{GeV}$, expected to be the track matched to the muon. L2: A medium-quality muon with $p_{T}>3 \mathrm{GeV}$ and tight scintillator timing cuts, OR an STT track with $p_{T}>20 \mathrm{GeV}$.

- MUHI3- L1: A muon in the wide region $(|\eta|<1.6)$, tight scintillator timing and tight wire requirements, matched to a CTT track with $p_{T}>13$ GeV. L2: A medium-quality muon with $p_{T}>3 \mathrm{GeV}$ and tight scintillator timing cuts, OR an STT track with $p_{T}>20 \mathrm{GeV}$.

At level 1, all single muon triggers, and indeed all triggers used in physics analyses, also include the condition that there is an active beam crossing so as to not trigger on noise.

The L3 options are as follows:

- ILM15- An isolated (isolated with respect to calorimeter energy) loosequality muon with $p_{T}$ (measured in the muon system) $>15 \mathrm{GeV}$.

- TK12_TLM12- A track-matched loose-quality muon with $p_{T}$ (measured from the track) $>12 \mathrm{GeV}$.

- ITLM10- A track-matched, isolated (isolated with respect to calorimeter energy) loose-quality muon with $p_{T}$ (measured from the track) $>10$ $\mathrm{GeV}$.

The full list of single muon triggers is in table 3.1.

\begin{tabular}{|lll|}
\hline MUHI1_ITLM10 & MUHI1_TK12_TLM12 & MUHI1_ILM15 \\
MUHI2_ITLM10 & MUHI2_TK12_TLM12 & MUHI2_ILM15 \\
MUHI3_ITLM10 & MUHI3_TK12_TLM12 & MUHI3_ILM15 \\
\hline
\end{tabular}

Table 3.1: List of single muon triggers used in the analysis. The field before the first underscore is label for the L1/L2 term, and the field after the first underscore is the L3 term. 


\subsubsection{Muon + Tau Triggers}

Muon+hadronic tau (hereafter $\mathrm{Mu}$-Tau or MTA) triggers complement the single muon triggers as they have looser muon thresholds; the hadronic tau requirement reduces the trigger rate so that the muon thresholds can be lowered, leading to increased muon efficiency. MTA triggers use the tracking, calorimeter, and muon systems to create the muon and tau objects. Like the single muon triggers they have different L1, L2, and L3 requirements that can be combined to make triggers. This analysis is the first instance of the specific use of a trigger with a hadronic tau component at $\mathrm{D} \varnothing$.

There are four different L1/L2 thresholds used in the selected MTA triggers. They are:

- MTA1- L1: A muon in the "all" region $(|\eta|<2.0)$ with tight scintillator and tight wire requirements, with no track match requirement. Also one L1 jet with $E_{T}>20 \mathrm{GeV}$ and $|\eta|<3.2$. L2: One medium quality muon with tight scintillator timing cuts (no $p_{T}$ requirement), and one tau object with $E_{T}>10 \mathrm{GeV},|\eta|<2.4$, and a $E_{\text {seed }+N N} / E_{3 \times 3}$ ratio (Sec. 3.1.2) of at least 0.5 .

- MTA3- L1: A muon in the "all" region $(|\eta|<2.0)$ with tight scintillator and tight wire requirements, with no track match requirement. Also one L1 tau with $E_{T}>8 \mathrm{GeV},|\eta|<2.4$, and $E_{2 \times 2} / E_{4 \times 4}$ ratio $>0.6$ (Sec. 3.1.1). At the beginning of RunIIb the trigger also required one L1CTT track with $p_{T}>5 \mathrm{GeV}$, but as of trigger list version 15.50 that has been replaced with a L1CalTrack object; a $5-\mathrm{GeV}$ track matched to an $8 \mathrm{GeV}$ jet. L2: One medium quality muon with tight scintillator timing cuts (no $p_{T}$ requirement), and one tau object with $E_{T}>10 \mathrm{GeV},|\eta|<2.4$, and a $E_{\text {seed }+N N} / E_{3 \times 3}$ ratio (see sec. 3.1.2) of at least 0.6.

- MTA4- L1: A muon in the "wide" region $(|\eta|<1.6)$ with tight scintillator and loose wire requirements, matched to a CTT track with $p_{T}>8$ $\mathrm{GeV}$. Also one L1 tau with $E_{T}>20 \mathrm{GeV}$ and $|\eta|<3.2$, and $E_{2 \times 2} / E_{4 \times 4}$ ratio $>0.5$. L2: One medium quality muon with tight scintillator timing cuts (no $p_{T}$ requirement), and one tau object with $E_{T}>10 \mathrm{GeV}$, $|\eta|<2.4$, and a $E_{\text {seed }+N N} / E_{3 \times 3}$ ratio (see sec. 3.1 .2 ) of at least 0.5.

- MTA6- L1: A muon in the "wide" region $(|\eta|<1.6)$ with tight scintillator and loose wire requirements, matched to a CTT track with $p_{T}>8$ $\mathrm{GeV}$. Also one L1 tau with $E_{T}>8 \mathrm{GeV},|\eta|<2.4$, and $E_{2 \times 2} / E_{4 \times 4}$ ratio $>$ 0.6. At the beginning of RunIIb the trigger also required one L1CTT track with $p_{T}>5 \mathrm{GeV}$, but as of trigger list version 15.50 that has been 
replaced with a L1CalTrack object; a $5-\mathrm{GeV}$ track matched to an $8 \mathrm{GeV}$ jet. L2: One medium quality muon with tight scintillator timing cuts (no $p_{T}$ requirement), and one tau object with $E_{T}>10 \mathrm{GeV},|\eta|<2.4$, and a $E_{\text {seed }+N N} / E_{3 \times 3}$ ratio (see sec. 3.1.2) of at least 0.6.

The level 3 terms for the MTA triggers are as follows:

- ITLM5T10NN1 An isolated, track-matched loose-quality muon with $p_{T}>5 \mathrm{GeV}$, and one tau with $|\eta|<3.0, p_{T}>10.0 \mathrm{GeV}$, and a level 3 tau neural network value L3TAUNN $>0.1$.

- ITLM5T5NN3 An isolated, track-matched loose-quality muon with $p_{T}>5 \mathrm{GeV}$, and one tau with $|\eta|<3.0, p_{T}>5.0 \mathrm{GeV}$, and L3TAUNN $>0.3$.

- ILM10T10NN1 An isolated, loose-quality muon with (local muon system) $p_{T}>10 \mathrm{GeV}$, and one tau with $|\eta|<3.0, p_{T}>10.0 \mathrm{GeV}$, and L3TAUNN $>0.1$.

- ILM10T5NN3 An isolated, loose-quality muon with (local muon system) $p_{T}>10 \mathrm{GeV}$, and one tau with $|\eta|<3.0, p_{T}>5.0 \mathrm{GeV}$, and L3TAUNN $>0.3$.

- TLM10T10NN1 A track-matched loose-quality muon with (track) $p_{T}>$ $10 \mathrm{GeV}$, and one tau with $|\eta|<3.0, p_{T}>10.0 \mathrm{GeV}$, and L3TAUNN $>0.1$.

- TLM10T5NN3 A track-matched loose-quality muon with (track) $p_{T}>$ $10 \mathrm{GeV}$, and one tau with $|\eta|<3.0, p_{T}>5.0 \mathrm{GeV}$, and a level 3 tau neural network value L3TAUNN $>0.3$.

By combining each L1/L2 possibility with each L3 possibility, we have 24 MTA triggers used in this analysis, shown in table 3.2.

\subsection{Trigger Efficiency Determination}

\subsubsection{Single Muon Triggers}

The so-called tag-and-probe method is the usual method to calculate muon trigger efficiencies. One selects a low-background sample of $Z \rightarrow \mu \mu$ data events (usually by requiring the di-muon invariant mass to be consistent with

the $Z$ mass) and chooses one of the two muons to be the "tag" muon. This muon must then fire some other trigger that is unbiased with respect to the 


\begin{tabular}{|ccc|}
\hline MTA1_TLM10T10NN1 & MTA1_ILM10T10NN1 & MTA1_ITLM5T10NN1 \\
MTA3_TLM10T10NN1 & MTA3_ILM10T10NN1 & MTA3_ITLM5T10NN1 \\
MTA4_TLM10T10NN1 & MTA4_ILM10T10NN1 & MTA4_ITLM5T10NN1 \\
MTA6_TLM10T10NN1 & MTA6_ILM10T10NN1 & MTA6_ITLM5T10NN1 \\
MTA1_TLM10T5NN3 & MTA1_ILM10T5NN3 & MTA1_ITLM5T5NN3 \\
MTA3_TLM10T5NN3 & MTA3_ILM10T5NN3 & MTA3_ITLM5T5NN3 \\
MTA4_TLM10T5NN3 & MTA4_ILM10T5NN3 & MTA4_ITLM5T5NN3 \\
MTA6_TLM10T5NN3 & MTA6_ILM10T5NN3 & MTA6_ITLM5T5NN3 \\
\hline
\end{tabular}

Table 3.2: List of Mu+Tau (MTA) triggers used in the analysis.

other (probe) muon. The other muon is the probe muon, and one tests whether or not there are trigger objects that pass the trigger requirements overlapping this offline muon, and then parametrize the fraction of muons with overlapping trigger objects (the efficiency) as a function of one or more kinematic variables. In this analysis we parametrize the efficiency as a function of muon $p_{T}, \eta$, and $\phi$. One can do this for any individual trigger, or for an "OR" of several triggers as was done in [32]. This is typically done with the program muo_cert, designed to match the trigger objects to data muons, and compute the efficiency of individual trigger terms.

\subsection{2 $\mathrm{Mu}+$ Tau Triggers}

To calculate the MTA trigger efficiency we use the tag-and-probe method to calculate the efficiency of the muon part of the trigger, and a pseudo-tag-andprobe procedure has been developed for the tau part. In the pseudo-tag-andprobe method we prepare a sample of events with at least one good offline tau candidate and one muon, with $\Delta \phi(\mu, \tau)>2.7$. This sample is dominated by $Z \rightarrow \tau_{\mu} \tau_{\text {had }}$ events. We require that the event fire a muon trigger and that the muon candidate has matching trigger objects. In this way we remove any trigger bias on the tau candidate (i.e. we are sure something other than the tau object triggered the event) and we can then apply the usual probe method with the tau candidate as the probe, checking for trigger objects overlapping our tau candidate that also satisfy the tau portions of the triggers in Table 3.2. This method is not limited to MTA triggers; it can be applied to any trigger with a hadronic tau component. Once we have both the muon and tau efficiency, we can then say that the total efficiency is $P_{\mu} P_{\tau}$, or simply the product of the two efficiencies as we assume no correlation between the muon and tau. 


\subsubsection{Trigger ORing}

Due to the higher instantaneous luminosities of RunIIb individual triggers must have tight requirements to keep the accept rates under control. Choosing only one trigger will give a very low efficiency. A better strategy is to take the OR of several triggers, i.e. at least one of a set of triggers must fire, to increase event yields. As one can see in the previous section, the single muon triggers and MTA triggers are designed to complement each other. However, one must correctly take correlations between the different triggers into account.

In the case of two triggers, the probability of an event firing at least one of the triggers can be written as

$$
P_{O R}=P_{t r i g 1}+P_{t r i g 2}-P_{t r i g 1, t r i g 2}
$$

When $P_{t r i g 1}$ and $P_{t r i g 2}$ are measured independently and $P_{\text {trig1,trig } 2}$ is the probability to fire both triggers. But it rapidly becomes extremely complicated if adding more than a few triggers. Thus it is easier to start from scratch and measure the efficiency of the entire OR simultaneously, rather than try to measure each trigger individually and combine afterward.

\subsubsection{Single Muon OR}

To measure the single muon OR efficiency we follow the same procedure as [32]. The DØ software program muo_cert computes the efficiency for each trigger term in each trigger it is given, and then and then the terms are combined together to compute the probability that at least one trigger from a specified list has a match at all three trigger levels. Figure 3.3 shows the 1-D efficiency projections for each of the three variables in which we parametrize the efficiency: muon $p_{T}, \eta$, and $\phi$.

\subsection{5 $\mathrm{Mu}+\mathrm{Tau} \mathrm{OR}$}

To OR the $\mathrm{Mu}+$ Tau Triggers we first start with the muon portion; following the same procedure as the single muon OR, only substituting the appropriate trigger terms into muo_cert. Figure 3.4 shows the 1-D efficiency projections for each of the three variables in which we parametrize the efficiency: muon $p_{T}, \eta$, and $\phi$, the same variables as the single muon OR. For the tau portion we check each trigger condition simultaneously, and if any trigger has a match at all three levels, we accept tau candidate as a match. The fraction of tau candidates that match with trigger objects satisfying the tau conditions of at least one trigger is denoted as $P_{\tau O R}$. Figure 3.6 shows the tau object OR efficiency as a function of tau $\eta$ and $E_{T}$. Figure 3.7 shows the efficiency as 

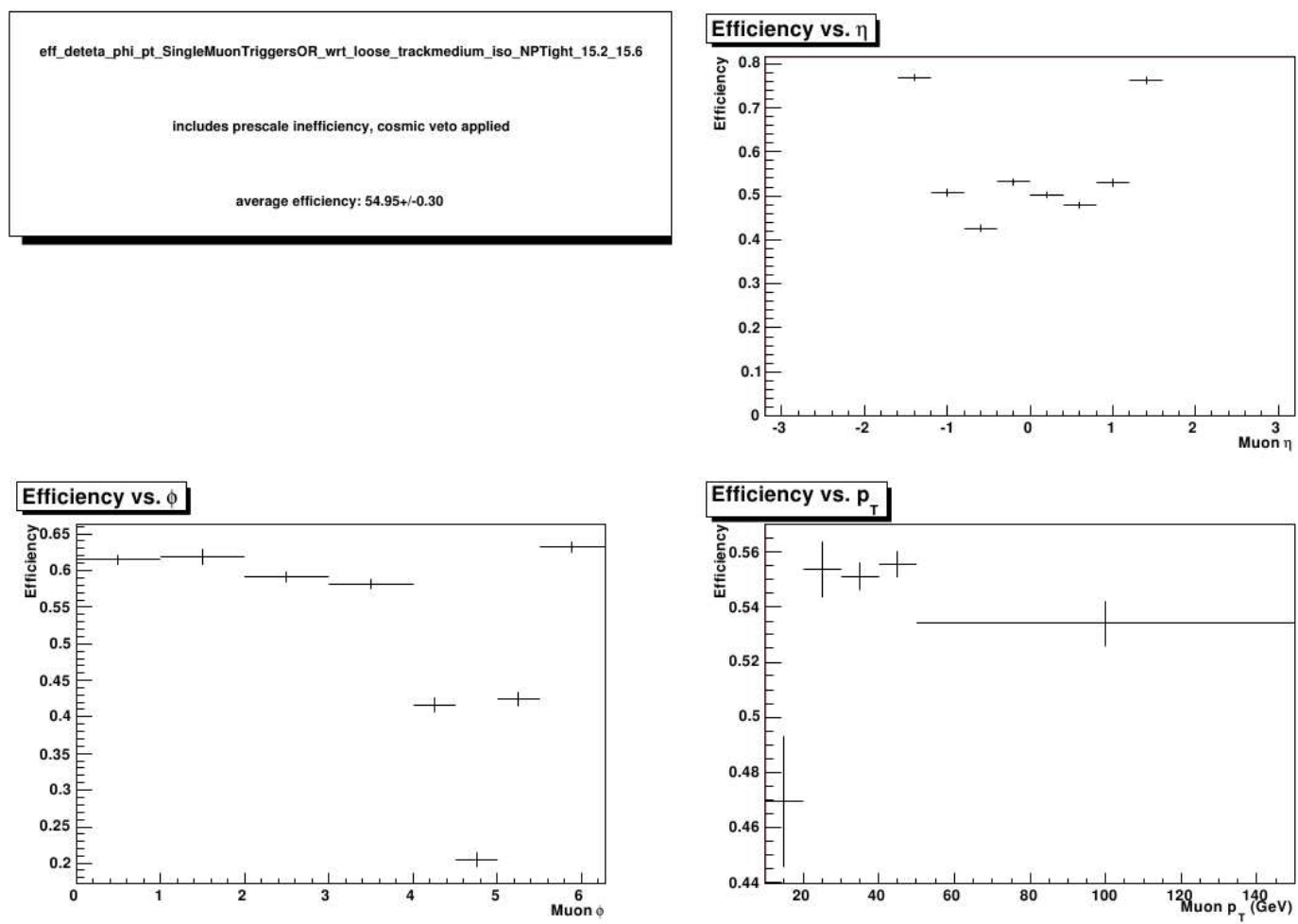

Figure 3.3: 1-D efficiency projections of the OR of the single muon triggers. Upper right: efficiency vs. detector $\eta$. Lower left: efficiency vs. $\phi$. Lower right: efficiency vs. $p_{T}$. The efficiency has been measured with respect to the same reconstructed muon quality cuts (loose muon, medium track match) as we make in the analysis. 
a function of tau $E_{T}$ for the OR, and for two individual MTA triggers. This clearly shows how the ORing is superior to choosing an individual trigger. The total efficiency of the or is then simply

$$
P_{M T A O R}=P_{\mu O R} P_{\tau O R}
$$
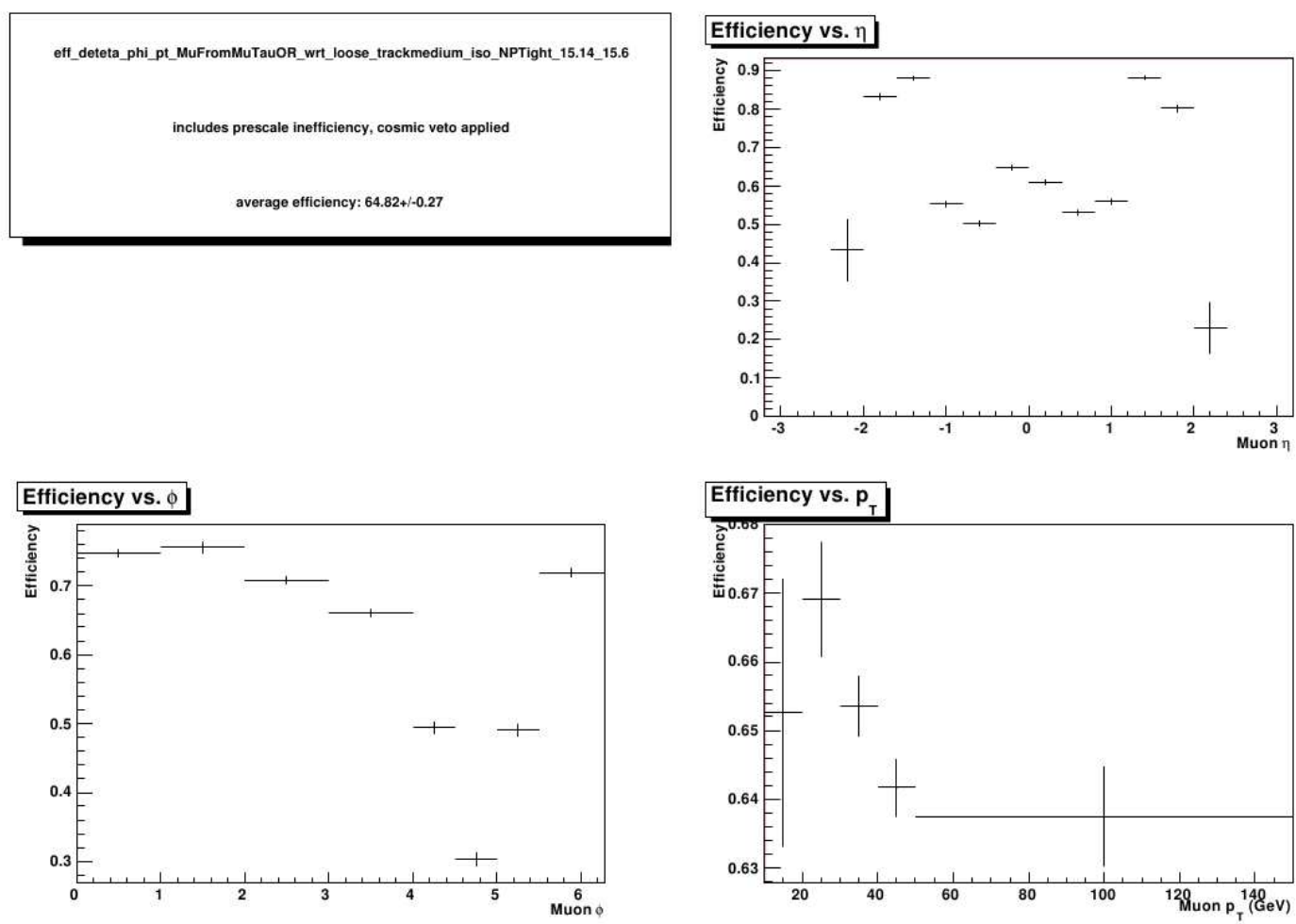

Figure 3.4: 1-D efficiency projections of the OR of muon terms from the MTA triggers. Upper right: efficiency vs. detector $\eta$. Lower left: efficiency vs. $\phi$. Lower right: efficiency vs. $p_{T}$. The efficiency has been measured with respect to the same muon quality cuts (loose muon, medium track match) as we make in the analysis.

\subsubsection{Totally Awesome OR (OR of ORs)}

To maximize signal acceptance we wish to combine all the triggers into an overarching OR. To take into account the correlation between the single muon OR and MTA OR we must also then measure the probability of an event to fire at least one single muon trigger and also at least one MTA trigger; we denote 
this probability as $P_{\text {single }} \mu O R, \mu O R$. Figure 3.5 shows the 1-D efficiency projections for $P_{\text {single } \mu O R, \mu O R}$. The combined efficiency for the full OR, $P_{F U L L O R}$, is then

$$
P_{\text {FULL OR }}=P_{\text {single } \mu O R}+P_{\mu O R} P_{\tau O R}-P_{\text {single } \mu O R, \mu O R} P_{\tau O R}
$$

where $P_{\text {single } \mu O R, \mu O R}$ is the probability to fire at least one trigger from the single muon OR and one muon requirement from the MTA triggers simultaneously. It is then this efficiency that we apply to the simulation to reflect the trigger requirement.
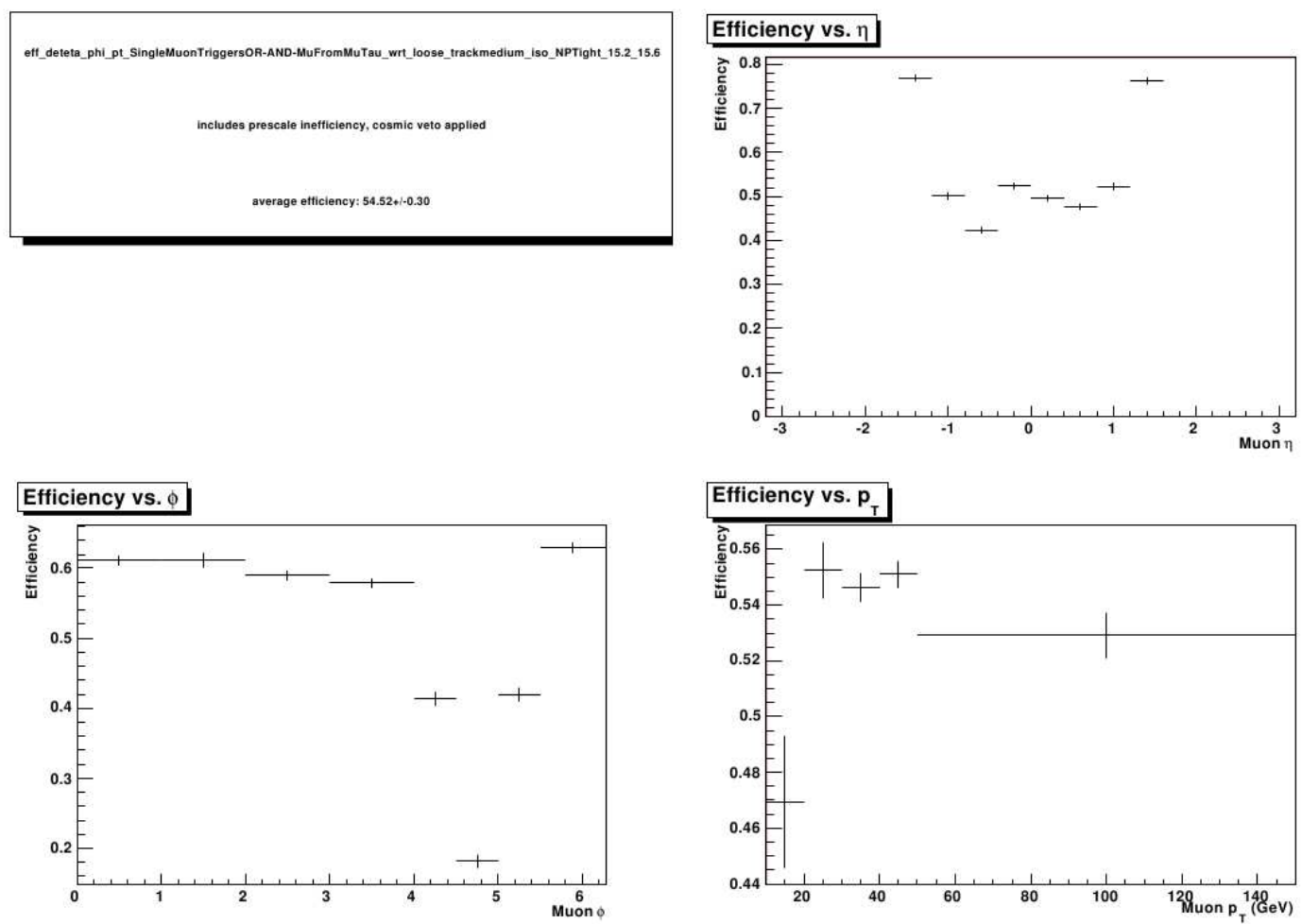

Figure 3.5: 1-D efficiency projections of firing at least one single muon trigger and at least one muon term from a MTA trigger. Upper right: efficiency vs. detector $\eta$. Lower left: efficiency vs. $\phi$. Lower right: efficiency vs. $p_{T}$. The efficiency has been measured with respect to the same muon quality cuts (loose muon, medium track match) as we make in the analysis. 


\section{Eta vs. pt}

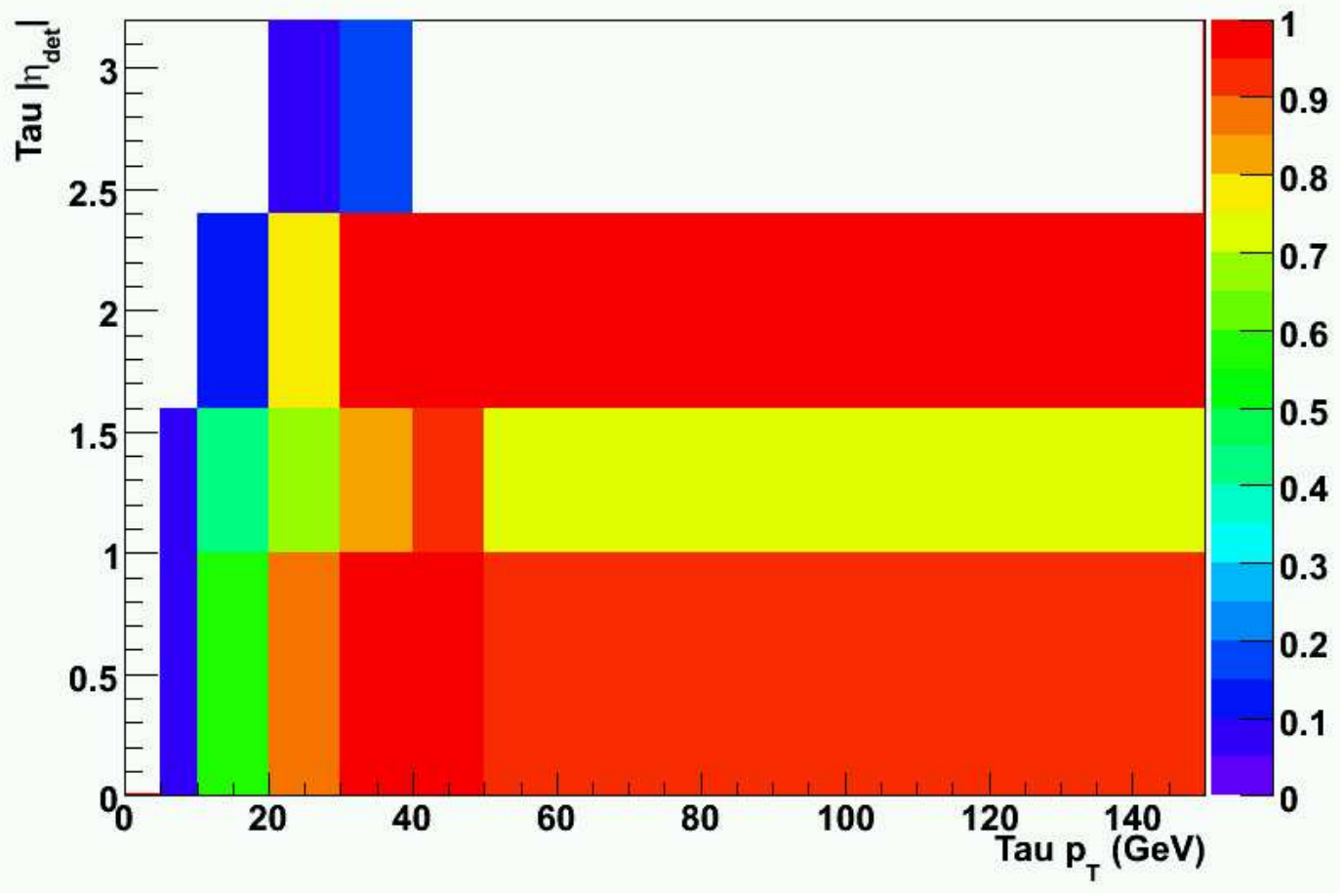

Figure 3.6: Efficiency of the OR of tau trigger terms parametrized in absolute value of detector $\eta$ (Y-axis) and $E_{T}$ (X-axis). 


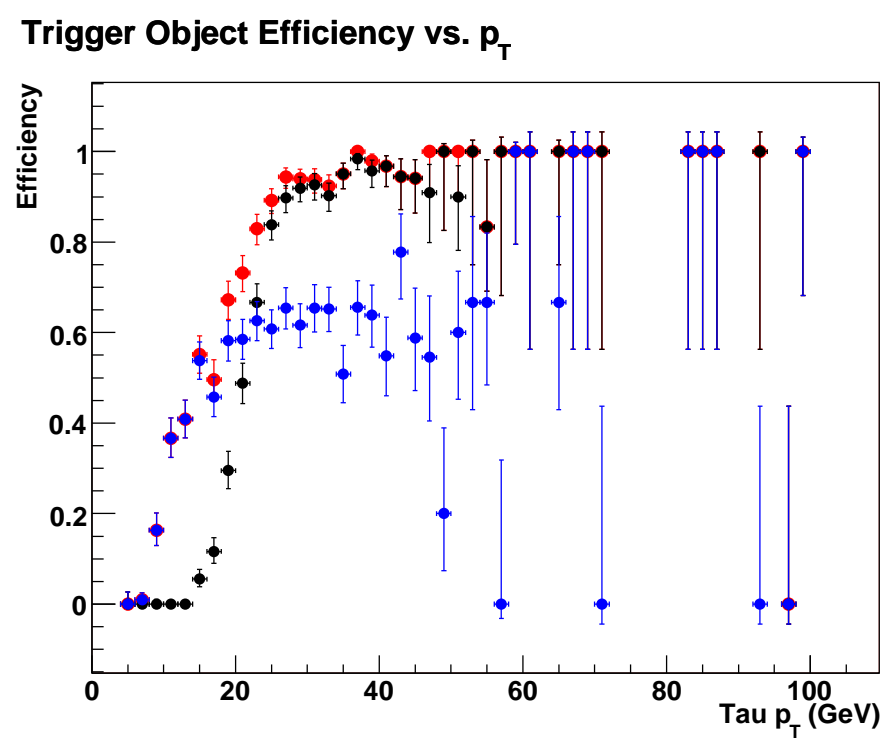

Figure 3.7: Efficiency vs. tau $E_{T}$ for type 2 taus (Sec. 4.1.5.) Red is the OR of the tau object conditions, Blue is the MTA3_TLM10T10NN1 trigger only, and black is the MTA1_TLM10T10NN1 trigger only. One can easily see that the two individual triggers are complimentary and the resulting efficiency improves over the entire range. 


\subsection{Data Acquisition}

If an event fires any trigger (i.e. passes all trigger requirements at all levels) DØ saves the event to tape. The event passes from the level 3 farm to the online system as shown in figure 3.8. Events from the L3 farm pass to collectors, which send the events to a datalogger and local PC host, which buffers the event on a hard disk, combining it with other events into larger files, and then eventually sending it to tape storage. The collectors also pass data to real-time data-quality monitoring processes (examines). Typically there are four such streams running during normal data taking, for a total of between 50 and 100 events to tape per second, depending on the instantaneous luminosity. Each event goes through only one stream. Finally the online system transfers these files from the disk buffer to the DLSAM system, an interface between the datalogger and the SAM (Sequential Access via Meta-data) system. Once in SAM events are available for later retrieval in an analysis. A diagnostic Secondary DAQ system (SDAQ) is also available and can be used for such tasks as real-time subdetector monitoring and calibration.

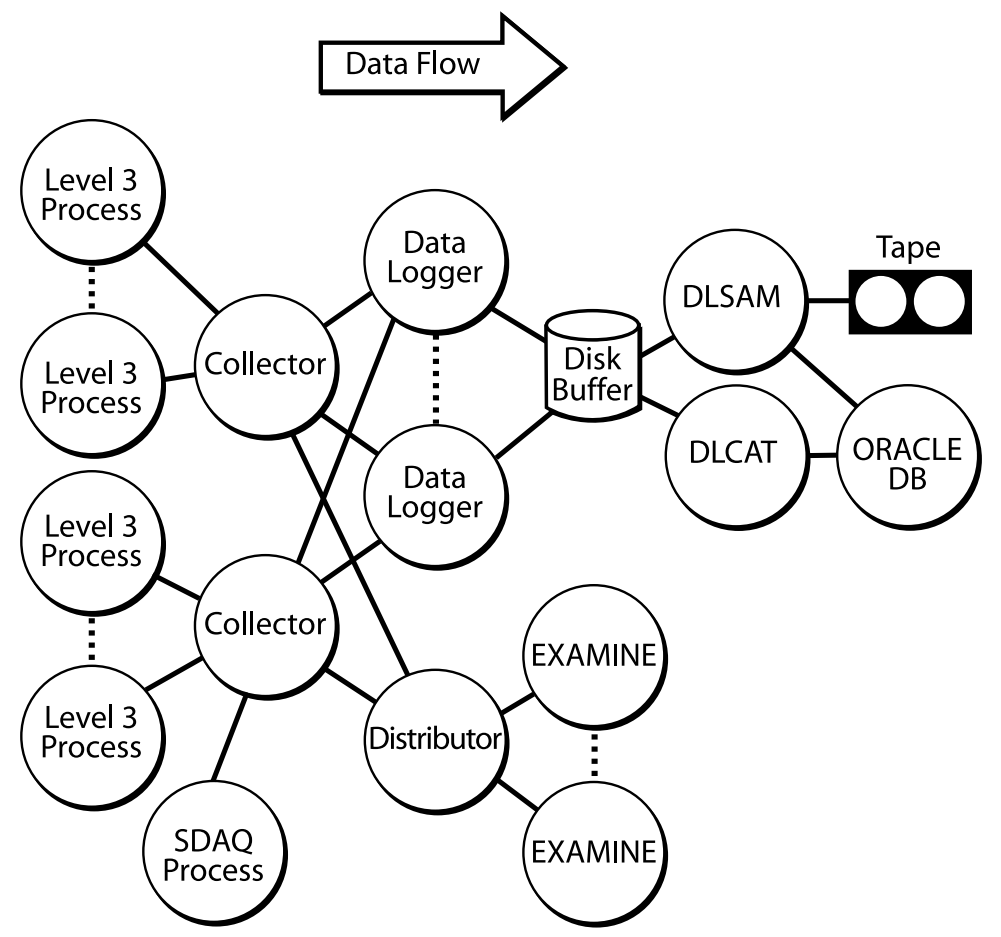

Figure 3.8: Dataflow through the DØ DAQ system [22]. 


\section{Chapter 4}

\section{Event Reconstruction and Object Identification}

In this chapter we discuss the details of event and physics object reconstruction at $\mathrm{D} \varnothing$, with special emphasis on the special procedures for the three objects in the final state of this analysis: hadronic taus, muons, and $b$-jets.

\subsection{Event Reconstruction}

Event reconstruction can be a time-consuming process, from several seconds to several minutes per event, as one must construct a variety of physics objects out of over one million individual channels. The D $\varnothing$ reconstruction program, RECO, reconstructs all physics objects from the raw data. Physics objects of interest to this analysis are tracks, primary vertices, muons, jets, and hadronic taus.

\subsubsection{Track Reconstruction}

DØ uses two algorithms to reconstruct charged tracks: the Histogramming Track Finder (HTF) [33] and the Alternative Algorithm (AA) [34]. In the HTF, hits from the tracking detectors go through a Hough transformation, which maps pairs of $(x, y)$ coordinates ${ }^{1}$ to a line in the $\rho-\phi$ plane. If there is a track, the $\rho-\phi$ lines from hits associated to the track will intersect at one point. The $\rho-\phi$ plane is binned into a 2-D histogram and populated by the detector hits. Then we discard histogram cells below a minimum hit multiplicity threshold and those with all hits contained in nearby cells. Then we convert the remaining cells to "templates" with a set of approximate track

\footnotetext{
${ }^{1}$ One being the hit position, the other being the origin.
} 
parameters; these templates go to a Kalman filter [35] for further cleaning and fitting, along with adding $z$ information.

The Alternative Algorithm begins by looking for tracks with SMT hits in at least three layers. Any barrel or F-disk hit can be used as a starting point. The AA then looks for hits within $\Delta \phi<0.08$ of the seed hit that are in a layer of greater radius. If one is found, the algorithm continues through the outer layers. Once there are hits in at least three layers the AA fits the $(x, y)$ hit positions to a circle, and if the fit has $\chi^{2}<16$, curvature $<30 \mathrm{~cm}$ (i.e. $p_{T}>180$ $\mathrm{MeV}$ ) and passes within $2.5 \mathrm{~cm}$ of the beam spot, it accepts the candidate. Hits from additional layers are added to the fit to improve resolution as long as the $\chi^{2}$ does not increase by more than 16. The CFT is also searched for seed hits to find those tracks without SMT hits, but such tracks are required to be consistent with a primary vertex to counter the increased combinatorics coming from the fact that the CFT has no $z$ information. Finally, track candidates pass to a Kalman filter. The Kalman filter parses the track candidate list, takes missed hits into account, corrects for material interactions and the solenoid field, and then accepts or rejects tracks based on the number of hits, fit $\chi^{2}$, missed hits, and association with a vertex. The total track candidate list is the union of the HTF and AA candidate lists.

\subsubsection{Primary Vertex Reconstruction}

The primary vertex is nominally the point in space where the hard scatter from the $p \bar{p}$ collision takes place. Here we discuss vertex reconstruction as well as how to distinguish the primary vertex in an event from other vertices, called secondary vertices.

Vertex reconstruction occurs in three stages. The first stage is track selection and clustering. Tracks with $p_{T}>0.5 \mathrm{GeV}, \geq 2 \mathrm{SMT}$ hits (when the track $\eta-z$ position is within the fiducial SMT region) are considered. The clustering algorithm then groups together candidate tracks that have a $z$ position on the beam line within $2 \mathrm{~cm}$ of each other. The second stage is vertex fitting, which is itself a two-step process. In the first step clustered tracks are fit to a common vertex using a tear-down Kalman filter. If the fit $\chi^{2}$ is greater than 10, the fitter removes the track with the highest individual $\chi^{2}$. Then the next highest $\chi^{2}$ is removed, and so on until the overall vertex fit $\chi^{2}$ is below 10. In the second step, the clustered tracks are sorted by distance of closest approach (DCA) to the beam spot (as determined in the first pass.) Tracks must have a DCA significance $\left(|D C A| / \sigma_{D C A}\right)$ less than 5.0. They then pass to an adaptive Kalman fitter [36] that re-weights each track's $\chi^{2}$ contribution 
to the fit according to:

$$
w_{i}=\frac{1}{1+e^{\left(\chi_{i}^{2}-\chi_{c u t o f f}^{2}\right) / 2 T}}
$$

Where $\chi_{i}^{2}$ is the i-th track's contribution to the fit $\chi^{2}, \chi_{\text {cutoff }}^{2}$ is the cutoff value (where the $w$ function has a value of 0.5 ), and $\mathrm{T}$ is the "temperature," or simply the sharpness of the function. This has the advantage of eliminating the need to cut on $\chi^{2}$ during fitting, resulting in less bias from heavy-flavor events where the tracks have low significance or are far away[36], [37].

The third stage of reconstruction is to separate the primary vertex from the other vertices in the event (either minimum bias interactions or secondary vertices. To this end, we create a probability distribution for a track to be associated to a minimum bias vertex based on the track's $p_{T}[38]$ :

$$
P\left(p_{T}\right)=\frac{\int_{\log _{10}\left(p_{T}\right)}^{\infty} F\left(p_{T}\right) d p_{T}}{\int_{\log _{10}(0.5)}^{\infty} F\left(p_{T}\right) d p_{T}}
$$

where $F\left(p_{T}\right)$ is the $\log _{10} p_{T}$ spectrum of tracks from minimum bias vertices, as determined from min bias Monte Carlo. The probability for a vertex to be a min bias vertex can then be written as

$$
P M B=\Pi \sum_{k=0}^{N-1} \frac{-\ln (\Pi)}{k !}
$$

Where $\Pi$ is the product of the individual $P_{p_{T}}$ values for each of the tracks associated with the vertex, and $k$ runs over the associated tracks. The vertex with the lowest $P M B$ value is the primary vertex [38].

\subsubsection{Muon Reconstruction}

Muons reconstruction uses information from the muon scintillators, wire chambers, and the central tracker. Reconstruction occurs in two stages; segment pattern recognition and scintillator matching. The segment pattern recognition consists of taking drift chamber hits in the three $A, B, C$ segments that are in time with a beam crossing. The hit timing constrains the hit position to a circle on the muon system wire. All pairs of hits that do not lie within the same drift circle can be fit to straight lines. Scintillator hits can also be added by extrapolating their position into the drift segment.

Using the toroid magnet one can obtain a $p_{T}$ measurement from the muon system by fitting the hits in the muon system to curved trajectories. Those fits that converge undergo a transformation of their $p_{T}$ and $A$ layer position 
information into tracking parameters, and then can be compared to central reconstructed tracks. Only tracks with $p_{T}>1 \mathrm{GeV}$ and $\Delta \eta, \Delta \phi$ between the track and transformed muon position less than 1 can be matched. If there is a track match, new muon parameters are calculated using an average of the local muon $p_{T}$ measurement and the track $p_{T}$ measurement, weighted by their uncertainties.

\subsubsection{Jet Reconstruction}

Jets come from calorimeter information and use several methods for reconstruction. The first step is to apply the T42 algorithm to remove noisy cells [39]. The T42 algorithm requires that the cell energy be at least $4 \sigma$, where

$\sigma$ is the RMS fluctuation in the energy, or to be $2.5 \sigma$ with a $4 \sigma$ neighboring cell. After that the simple cone algorithm [40] runs to create calorimeter preclusters with a radius of $R=0.3$. The preclusters are seeds for the RunII cone algorithm [40] which makes jets from the calorimeter cells and towers with a cone size of $R=0.5$. There is a minimum $E_{T}$ threshold of $6 \mathrm{GeV}$ for reconstruction. When selecting jets for this analysis we apply the standard set of quality requirements at $\mathrm{D} \varnothing$ in addition to the analysis-specific $E_{T}$ and $\eta$ requirements. The requirements come on the corrected jets, i.e. after applying all data/MC scale factors (sec. 5.3.3) and the Jet Energy Scale (both data and $\mathrm{MC})$ to the raw measured jets.

\section{Jet Energy Scale}

The most significant correction to the raw calorimeter-measured energy is the Jet Energy Scale (JES) [41]. It takes detector effects into account to give a more accurate representation of the true parton/shower energy. The general form of the correction is

$$
E_{\text {jet }}^{\text {particle }}=\frac{E_{\text {jet }}^{\text {calo }}-O}{F_{\eta} \times R \times S}
$$

Where $E_{\text {jet }}^{\text {calo }}$ is the jet energy as measured in the calorimeter; $O$ is the offset parameter, representing the contribution from electronics noise, radioactive decay of uranium in the calorimeter, min bias interactions, and multiple scattering; $F_{\eta}$ is the relative response correction (to insure a uniform response as a function of detector $\eta$ ). The relative response function is measured by the Missing Transverse Energy Projection Fraction (MPF) method. $R$ is the response parameter, reflecting the detector's response to energy (i.e. how much of the true energy is measured by the detector); $S$ is the showering parameter, reflecting jet energy that is lost outside the cone. $F_{\eta}$ and $R$ are measured 
in unbiased $\gamma+$ jets and di-jet events. One measures $S$ by measuring the jet energy density as a function of the distance in $\eta-\phi$ space from the jet axis in both data and Monte Carlo. Figure 4.1 shows the offset correction, figure 4.2 shows the relative response function, figure 4.3 shows the absolute response function, and figure 4.4 shows the showering correction.

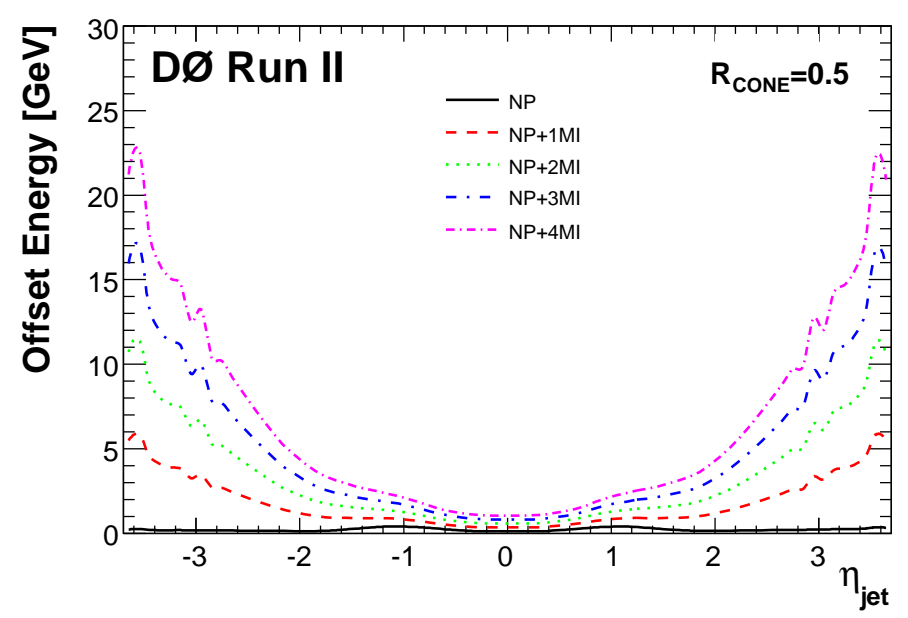

Figure 4.1: Offset correction to the jet energy scale for a jet cone size of 0.5 as a function of jet $\eta$ as measured from the center of the detector. The different curves represent different primary vertex multiplicities[41].

\subsubsection{Tau Reconstruction}

Hadronic taus consist of calorimeter clusters, electromagnetic calorimeter subclusters, and tracks [42]. The calorimeter clusters come from a simple cone algorithm (Sec. 4.1.4) of radius $R=0.3$, with an isolation radius of $R_{\text {iso }}=0.5$. Neutral pions $\left(\pi^{0}\right)$ from tau decays will deposit most of their energy in the electromagnetic portion of the calorimeter. The tau reconstruction algorithm searches for EM clusters reconstructed with the nearest-neighbor algorithm in the EM3 layer of the calorimeter. If such clusters are found, they are added to the tau, along with EM clusters in the other layers and any preshower hits.

Track association begins by making a $p_{T}$-sorted list of all tracks within a cone of 0.5 around the calorimeter cluster centroid[42]. A track must have $p_{T}>1.5 \mathrm{GeV}$ to be considered. If there is a suitable track, a second track is added if the invariant mass of the two tracks is less than $1.1 \mathrm{GeV}$, and the two tracks are within $2 \mathrm{~cm}$ in $z$ at the point of closest approach. A third track is added if it is within $2 \mathrm{~cm}$ in $z$ of the first (i.e. highest $p_{T}$ ) track at the point of 

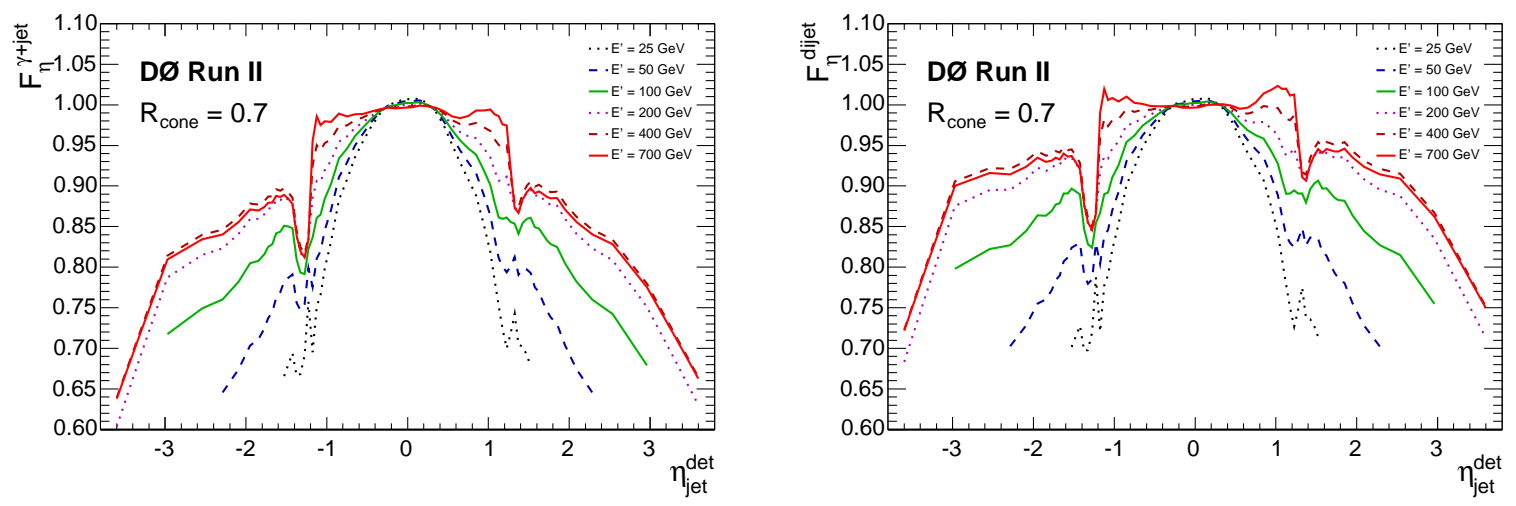

Figure 4.2: Relative response correction to the jet energy scale in a photon + jet sample (left) and di-jet sample (right) [41].

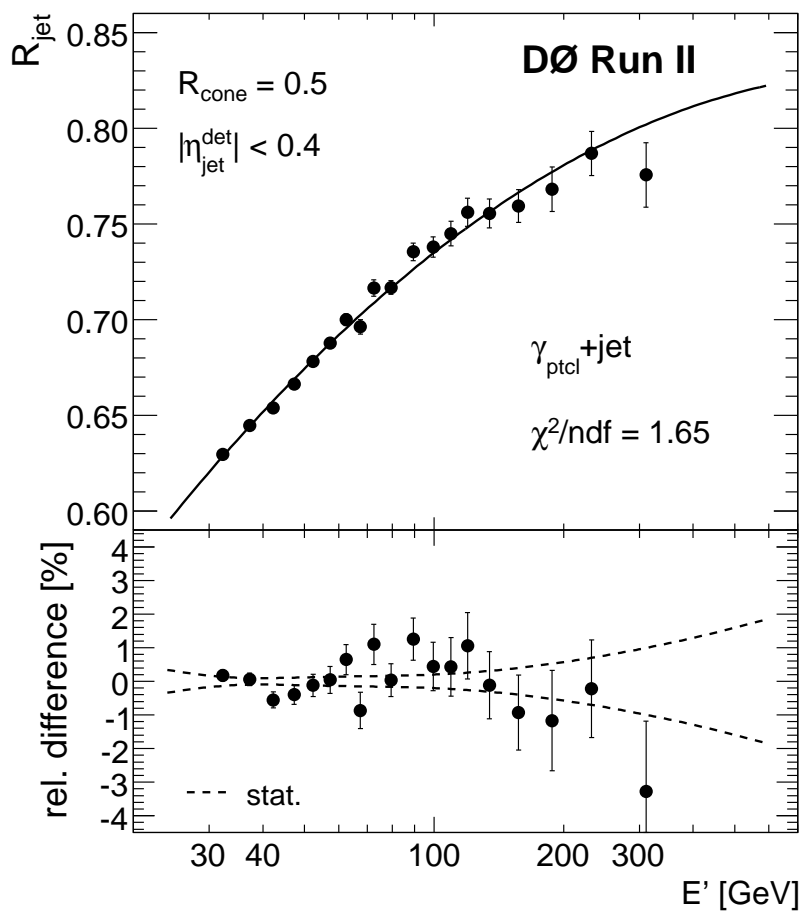

Figure 4.3: Absolute response correction to the jet energy scale for a jet cone size of 0.5 [41]. 


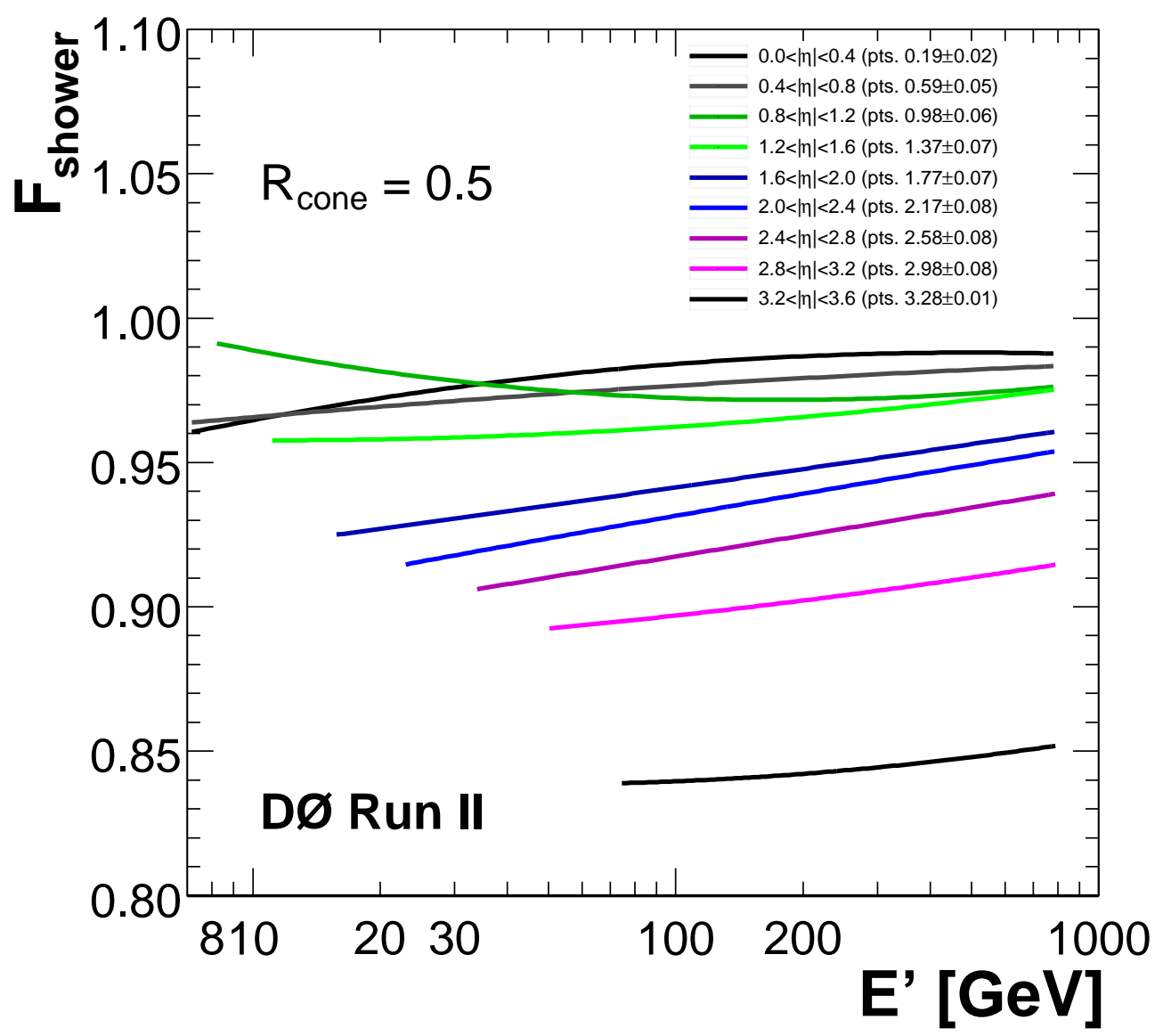

Figure 4.4: Jet showering correction to the jet energy scale as a function of jet energy for a jet cone size of 0.5 [41]. 
closest approach, the invariant mass of the three tracks is less than $1.7 \mathrm{GeV}$, and the sum of the three tracks' charges is either +1 or -1 . Note that this approach allows for tau candidates with two tracks that have a charge sum of zero; we remove such candidates at the analysis level.

At D $\varnothing$ reconstructed hadronic tau are then classified as one of three types depending on their detector signature:

- Type 1: A calorimeter cluster having no EM subclusters and one charged track match. Analogous to $\tau \rightarrow \pi^{ \pm} \nu$.

- Type 2: A calorimeter cluster having at least one EM subcluster with one charged track match. Analogous to $\tau \rightarrow \pi^{ \pm} \pi^{0} \nu$.

- Type 3: A calorimeter cluster with or without EM subclusters and at least two charged tracks. Analogous to a three-prong decay, or $\tau^{ \pm} \rightarrow$ $\pi^{ \pm} \pi^{ \pm} \pi^{\mp} \nu$.

Instrumental background from jets being misidentified as taus is large. Therefore additional techniques to separate real taus from non-tau hadronic jets and electrons are available; we discuss them in section 4.2.1.

\subsection{Object Identification}

\subsubsection{Tau-ID}

Hadronic jets and electrons can be misidentified as a hadronic tau. To identify real taus and reject fakes a neural network $\left(N N_{\tau}\right)$ has been developed [43]. There is a separate neural net for each of the three tau types. A total of twelve variables are used for training, but not all variables are used for each tau type; full details are in [43]. The training sample is $Z / \gamma \rightarrow \tau \tau\left(M_{Z}=130-250\right.$ $\mathrm{GeV}) \mathrm{MC}$ and the testing, or validation, signal is $Z / \gamma \rightarrow \tau \tau\left(M_{Z}=60-130\right.$ $\mathrm{GeV}) \mathrm{MC}$. The background sample is data events with an anti-isolated muon and tau candidate (this sample is dominated by fake taus). The background testing/validation sample is data events with an anti-isolated muon and tau candidate having the same sign as the muon. Figures 4.5,4.6, and 4.7 show the $N N_{\tau}$ output for data, $W \rightarrow \mu \nu \mathrm{MC}$, and $Z \rightarrow \tau \tau \mathrm{MC}$ for the three tau types. We see that samples dominated by fake taus (anti-isolated muon events, $W$ $\mathrm{MC})$ have a low neural net output, while events with real taus $(Z \rightarrow \tau \tau)$ have a high $N N_{\tau}$ value. 

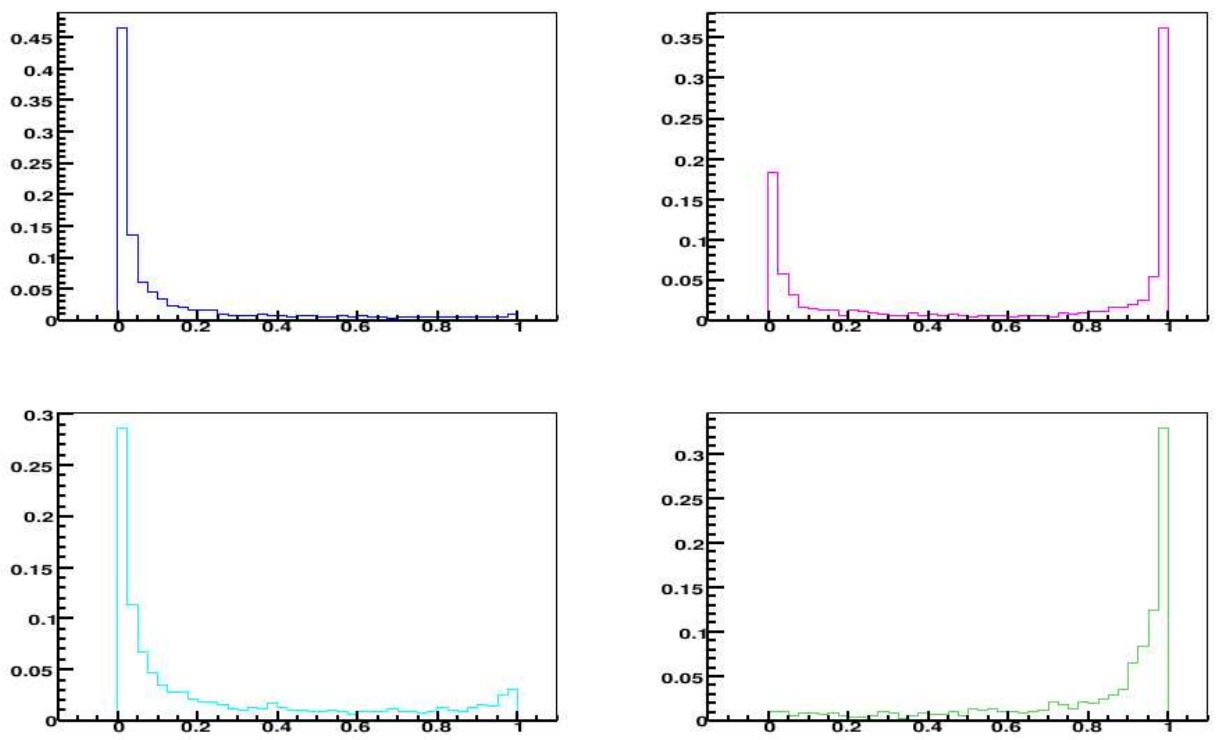

Figure 4.5: $N N_{\tau}$ Output for type 1 taus in data (upper left), data with muontau overlaps (upper right), $W \rightarrow \mu \nu \mathrm{MC}$ events (lower left), and $Z \rightarrow \tau \tau \mathrm{MC}$ events (lower right)[43].
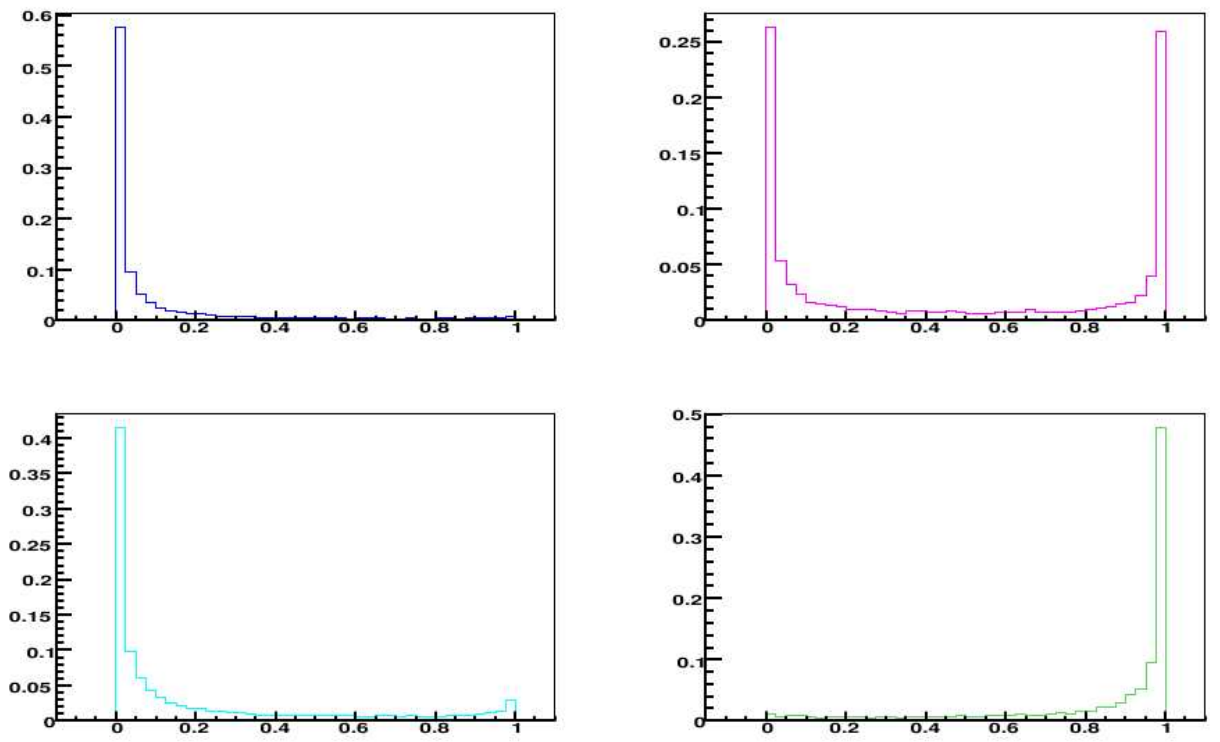

Figure 4.6: $N N_{\tau}$ Output for type 2 taus in data (upper left), data with muontau overlaps (upper right), $W \rightarrow \mu \nu \mathrm{MC}$ events (lower left), and $Z \rightarrow \tau \tau \mathrm{MC}$ events (lower right)[43]. 

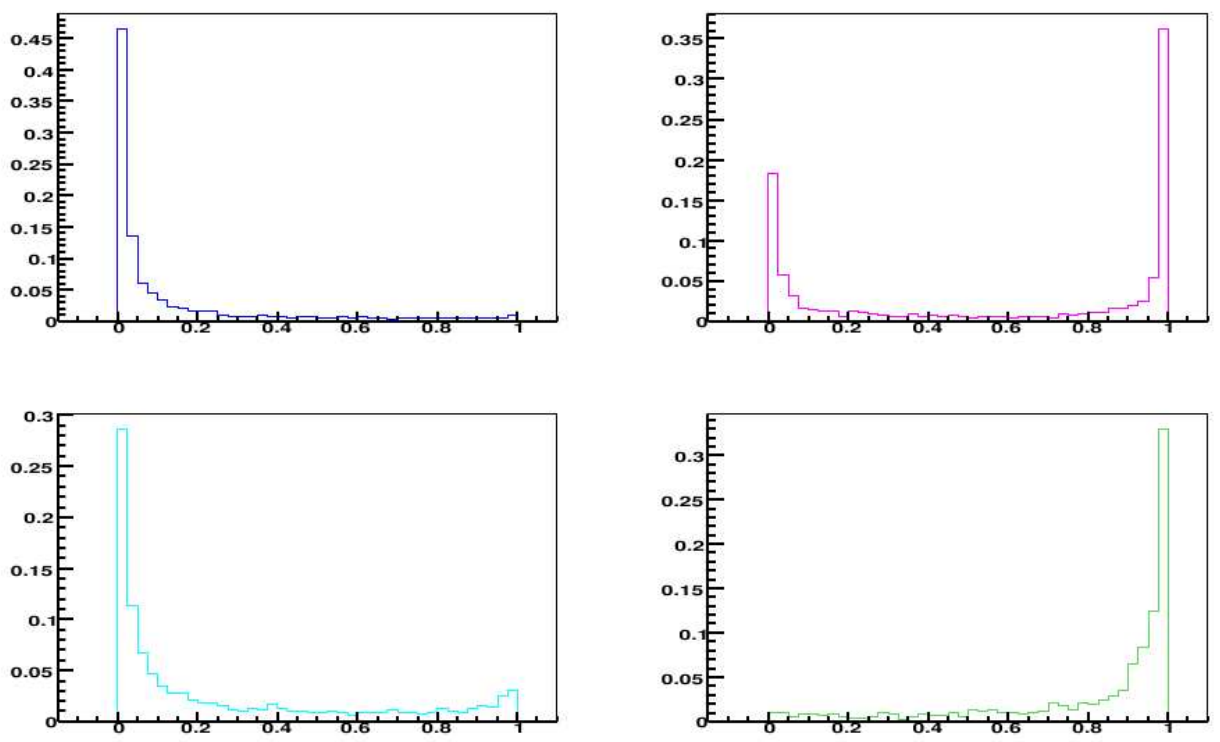

Figure 4.7: $N N_{\tau}$ Output for type 3 taus in data (upper left), data with muontau overlaps (upper right), $W \rightarrow \mu \nu \mathrm{MC}$ events (lower left), and $Z \rightarrow \tau \tau \mathrm{MC}$ events (lower right)[43].

\subsubsection{Muon-ID}

\section{Muon Type and Quality}

Muon identification and quality is determined by the presence of a track match, number of segments having hits, and track quality [44]. The hit patterns in the $A, B$, and $C$ layers determine the muon type, as shown in table 4.1. There are three possibilities for muon quality: loose, medium, and tight.

Tight muons have the following requirements:

- $\mathrm{nseg}=+3$

$\bullet 2$ A layer wire hits

- $\geq 1$ A layer scintillator hit

- $\geq 3$ BC layer wire hits

- $\geq 1$ BC scintillator hit

- a converged local fit $\left(\chi_{l o c}^{2}>0\right)$

medium nseg=3 muons have the following requirements: 


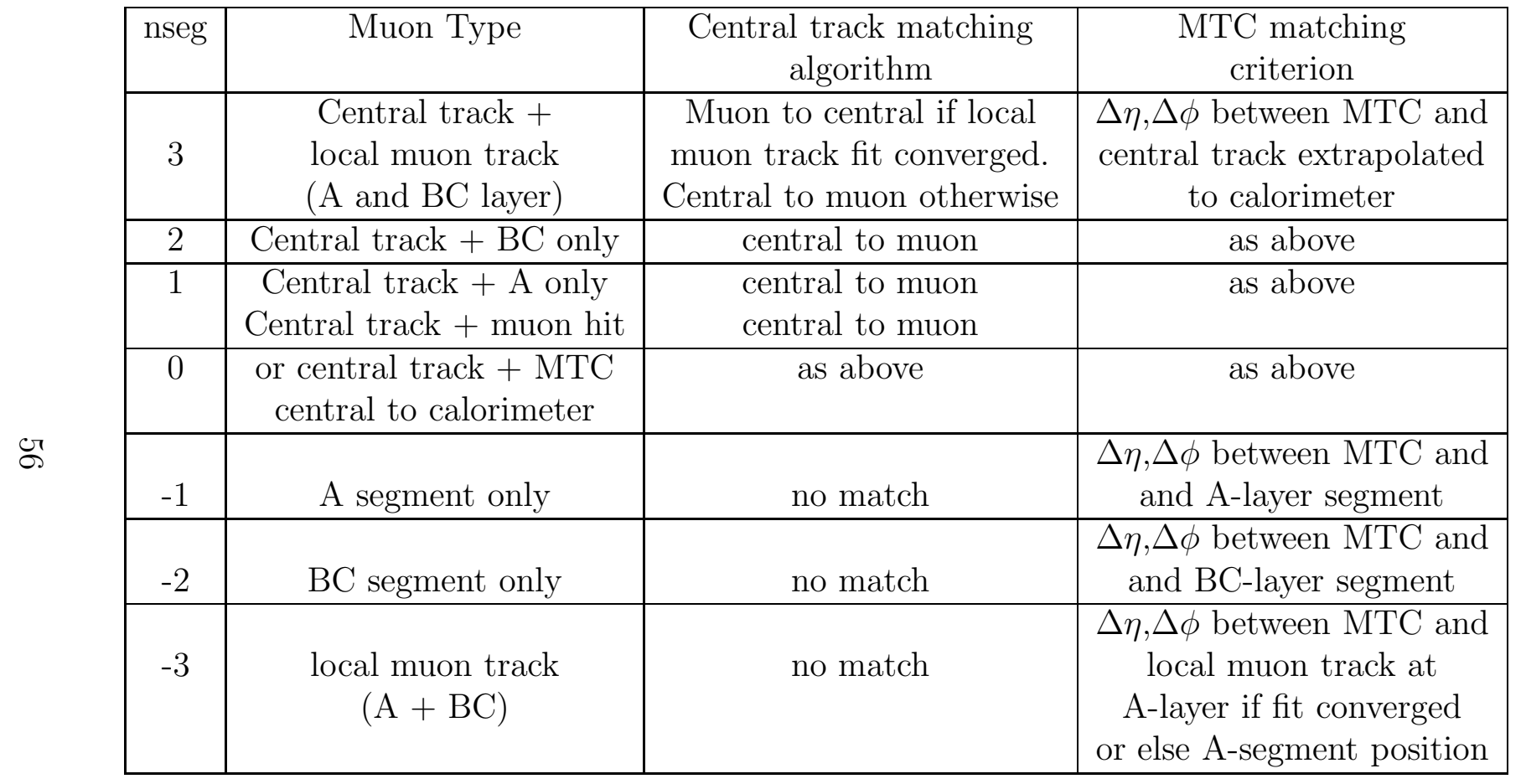

Table 4.1: Muon types and descriptions, based on nseg value[44]. MTC stands for Muon Tracking in the Calorimeter (not used in this analysis.) 
- $\geq 2$ A layer wire hits

- $\geq 1$ A layer scintillator hit

- $\geq 2$ BC layer wire hits

- $\geq 1 \mathrm{BC}$ scintillator hit (except for central muons with less than four BC wire hits).

Loose nseg $=3$ muons are as above, but allowing one of the tests to fail, with the A wire and scintillator requirement treated as one and requiring always at least one scintillator hit.

Loose nseg $=+2$ muons have the following requirements:

- central track match

- $\geq 1$ BC layer scintillator hit

- $\geq 2$ BC layer wire hits

Medium nseg $=2$ muons are as above, but located in the bottom part of the detector (octant 5 and 6 with $\left|\eta_{\text {det }}\right|<1.6$ ).

Loose nseg $=+1$ muons are as follows:

- central track match

- $\geq 2$ A layer wire hits

- $\geq 1$ scintillator hit

Medium nseg=1 muons are as above, but located in the bottom part of the detector (octant 5 and 6 with $\left|\eta_{\text {det }}\right|<1.6$ ).

\section{Muon Track Quality}

There are three classes for tracks matched to muons. They are also called loose, medium, and tight. A loose track is one with $|d c a|<0.02(0.2) \mathrm{cm}$ for tracks with(out) SMT hits. A medium track fulfills the loose requirements and also has $\chi^{2} /$ d.o.f. $<4$. A tight track meets the medium requirements and has at least one SMT hit. 


\section{Muon Isolation}

Muon isolation is one of the best ways to separate muons coming from the decay of massive objects and the otherwise overwhelming contribution of muons from multijet events with heavy flavor decay or pion and kaon decays in flight. The two variables used for the isolation determination are the Calorimeter Halo (later denoted as $I_{c a l}$ ), and TrackHalo (alternatively TrackCone), later denoted as $I_{t r k} . \quad I_{c a l}$ is defined as the calorimeter energy in an annulus of $0.1<R<0.4$ around the muon. $I_{t r k}$ is defined as the sum of the $p_{T}$ of tracks within $0.0<R<0.5$ of the muon, excluding the muon track itself.

\subsection{3 b-ID}

Identification of jets from $b$-quark decays is a critical component of this analysis. Since the $b$ quark has a lifetime of $\approx 1$ ps it will typically travel several millimeters before decaying, resulting in a displaced vertex, or a vertex that appears well-separated from the collision point, at the point of the $b$-decay. Tracks from such a vertex will have high impact parameter significance, key for identifying $b$-jets. Jets meeting certain criteria, such as being associated with such high-impact parameter tracks, can be identified, or "tagged", as $b$-jets.

This analysis uses the D $\varnothing$ Neural Network b-tagger [45],[46]. It is a neural network with seven input variables, listed in table 4.2. The variables are themselves quantities calculated within three other $b$-taggers: the Secondary Vertex Tagger (SVT), the Counting Signed Impact Parameters (CSIP) tagger, Jet LIfetime Probability Tagger (JLIP). The variables deal with tracks and/or Secondary vertices $(\mathrm{SVs})$, which are vertices not identified as the primary vertex (i.e. the main $p \bar{p}$ hard scatter.)

\begin{tabular}{|c|c|c|}
\hline Rank & Variable & Description \\
\hline 1 & $\mathrm{SVT}_{S L}$ DLS & Decay Length Significance of the Secondary Vertex (SV) \\
2 & CSIP Comb & Weighted combination of the tracks' IP significances \\
3 & $\mathrm{JLIP}$ Prob & Probability that the jet originates from the PV \\
4 & $\mathrm{SVT}_{S L} \chi^{2} /$ dof & Chi Square per degree of freedom of the SV \\
5 & $\mathrm{SVT}_{L} \mathrm{NT}$ tracks & Number of tracks used to reconstruct the SV \\
6 & $\mathrm{SVT}_{S L}$ Mass & Mass of the SV \\
7 & $\mathrm{SVT}_{S L}$ Num & Number of SV found in the jet \\
\hline
\end{tabular}

Table 4.2: NN b-tagger input variables ranked in order of power.[46]

There are two samples used together to measure $b$-tagging efficiency. One is a sample with a loose muon of $p_{T}>4 \mathrm{GeV}$ inside a jet with a cone size of 
0.7 (a "muonic" jet), and the other is a $b$-enriched subsample consisting of a muonic jet and an additional tagged jet. The so-called "System 8" (S8) method measures the tagger's efficiency. It is a system of eight equations and eight unknowns

$$
\begin{aligned}
n & =n_{b}+n_{u d s g c} \\
p & =p_{b}+p_{u d s g c} \\
n^{S L T} & =\epsilon_{b}^{S L T} n_{b}+\epsilon_{u d s g c}^{S L T} n_{u d s g c} \\
p^{S L T} & =\epsilon_{b}^{S L T} p_{b}+\epsilon_{u d s g c}^{S L T} p_{u d s g c} \\
n^{N N} & =\epsilon_{b}^{N N} n_{b}+\epsilon^{N N} n_{u d s g c} \\
p^{N N} & =\beta \epsilon^{N N} p_{b}+\alpha \epsilon_{u d s g c}^{N N} p_{u d s g c} \\
n^{S L T, N N} & =\kappa_{b} \epsilon_{b}^{S L T} \epsilon_{b}^{N N} n_{b}+\kappa_{u d s g c} \epsilon_{u d s g c}^{S L T} \epsilon_{u d s g c}^{N N} n_{u d s g c} \\
p^{S L T, N N} & =\kappa_{b} \beta \epsilon_{b}^{S L T} \epsilon_{b}^{N N} p_{b}+\kappa_{u d s g c} \alpha \epsilon_{u d s g c}^{S L T} \epsilon_{u d s g c}^{N N} p_{u d s g c}
\end{aligned}
$$

where $n$ is the number of jets in the muonic jet sample, $p$ is the number in the $b$-enriched sample and $\epsilon$ is the efficiency of the tagger, with the superscripts $N N$ and SLT referring to the $N N$ and SLT (Soft Lepton Tagger) ${ }^{2}$ taggers, respectively. $\alpha, \beta, \kappa_{b}, \kappa_{u d s c}$, and $p_{T \text { Rel }}$ are correlation coefficients. $\alpha$ is the ratio of tagging efficiencies on $u d s c$-jets in the two samples, $\beta$ is the ratio of the $b$-tagging efficiencies in the two samples, $\kappa_{b}$ is the correlation between the two taggers on $b$-flavored jets, $\kappa_{u d s c}$ is the correlations between the two taggers on $u d s c$-jets, while $p_{T R e l}$ is the ratio of the SLT tagging efficiencies on $c$ and $u d s$-jets. By solving the system, one can determine the efficiency of the NN tagger.

Several values of NN output have been chosen as "operating points", or fixed cut values intended for use in analysis. The optimal choice between these standard operating points is analysis-dependent. Analyses requiring multiple $b$-quarks might choose a looser cut to increase statistics, while those only requiring one $b$-quark might choose a tighter cut to gain a lower fake rate. This analysis uses the TIGHT operating point, corresponding to a NN cut of 0.775 on each jet. Figure 4.8 shows the $b$-tagging efficiency as a function of jet $p_{T}$ for this operating point, while Figure 4.9 shows the fake rate.

\footnotetext{
${ }^{2}$ An SLT tag is defined as a muon inside a jet having $p_{T \text { Rel }}>0.5 \mathrm{GeV}$, where $p_{T \text { Rel }}$ is the muon $p_{T}$ measured with respect to the jet axis.
} 

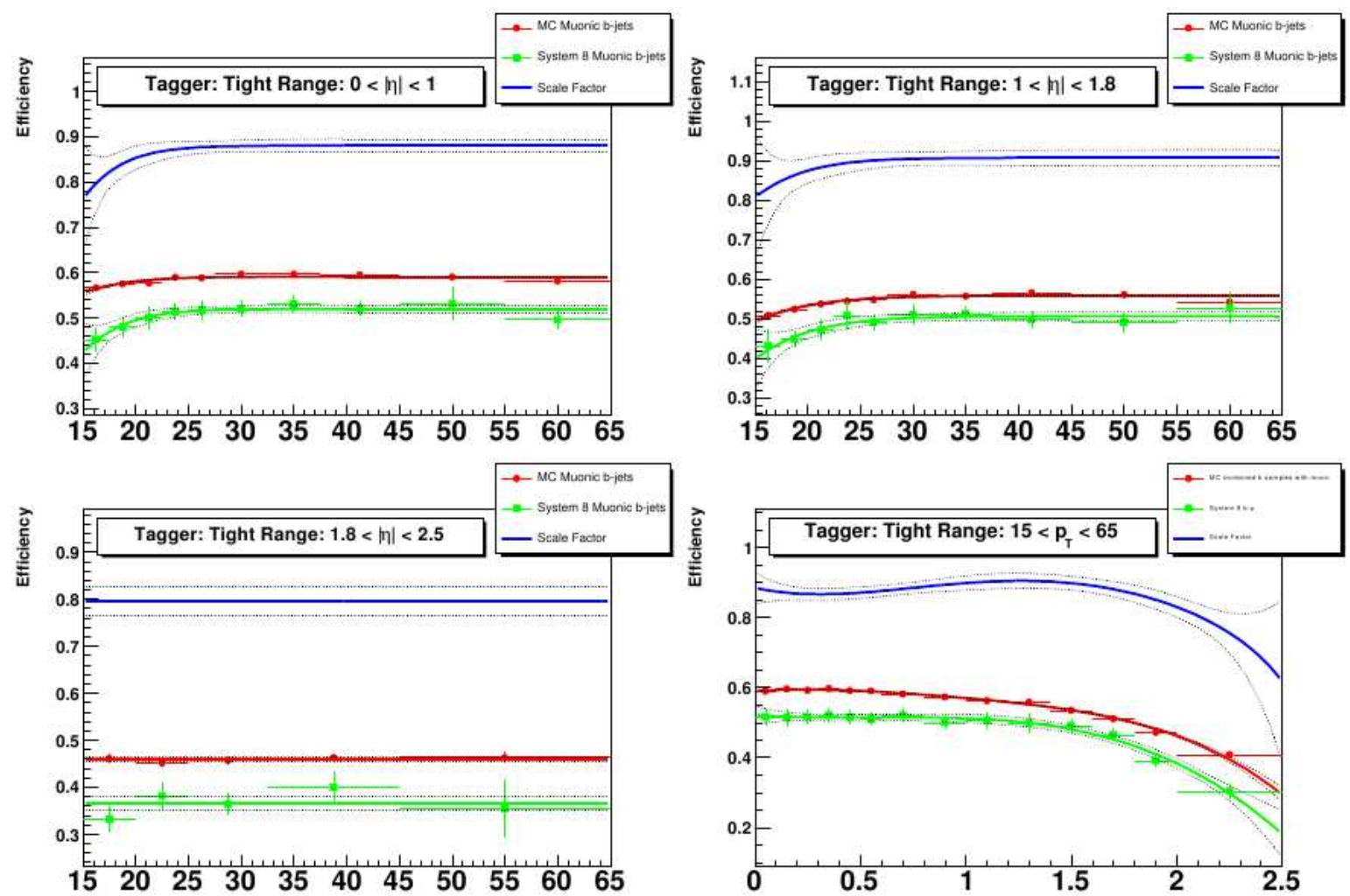

Figure 4.8: Efficiency of the NN $b$-tagger for the TIGHT operating point in $\mathrm{MC}$ (red) and data(green) as a function of jet $p_{T}$ in the $\mid \eta<1$, or CC, region (upper left); the $1<\mid \eta<1.8$, or Inter-Cryostat Region (ICR) (upper right); the $1.8<\mid \eta<2.5$, or EC, region (lower left); and as a function of $|\eta|$ (bottom right) [46]. 


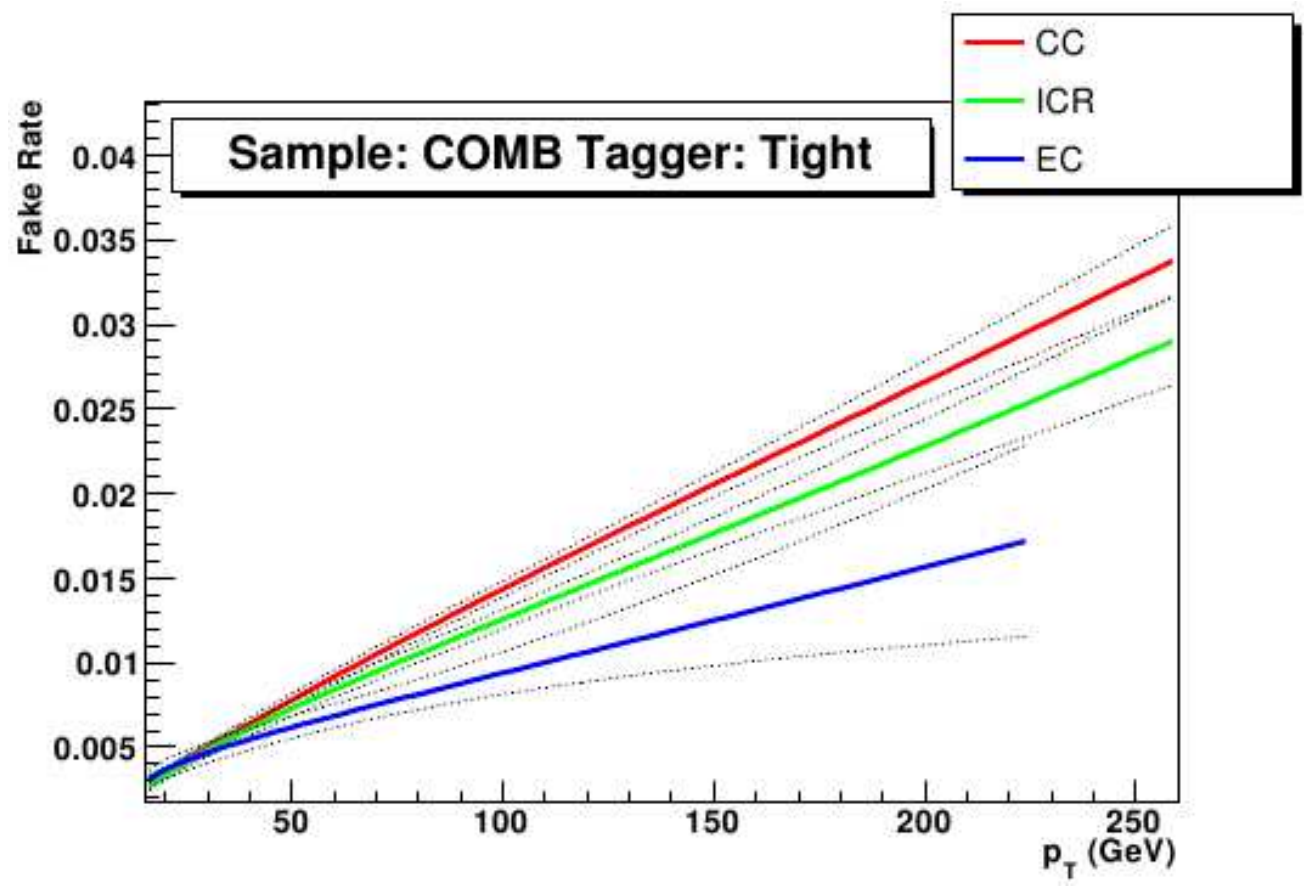

Figure 4.9: Fake tag rate of the NN $b$-tagger for the TIGHT operating point in $\mathrm{MC}(\mathrm{red})$ and data(green) as a function of jet $p_{T}$ in the $\mathrm{CC}$ (red), ICR(blue), and EC(green). The dashed lines represent the uncertainties [46]. 


\section{Chapter 5}

\section{Simulation}

In this chapter we discuss Monte Carlo (MC) simulation at $\mathrm{D} \varnothing$ along with the necessary tunes and corrections that we apply to make the simulation describe the data.

\subsection{Event Generators}

The Monte Carlo events in this analysis come from two main generators, Pythia[47] and Alpgen[48]. Pythia is a Fixed-Order Matrix Element (FOME) generator capable of simulating a wide range of scattering processes. Pythia uses CTEQ6 parton distribution functions (PDFs) [49]. Alpgen is also a FOME generator and provides a better description of events with multiple partons in the final state. The vector boson $(W, Z)+$ jets and $(W, Z)+$ heavy flavor $(c \bar{c}, b \bar{b})+$ jets Monte Carlo samples were generated with AlPGEN. In our case ALPGEN events contain a vector boson production/decay in association with a number $n$ of additional light partons(lp), typically ranging from 0 to 5 depending on the process. The $n$-lp samples taken together for each process comprise the full sample for that process. The AlPGEnoutput then passes to PythiA, which handles additional hadronization and showering. As PYTHIA can add a $b \bar{b}$ or $c \bar{c}$ pair during the hadronization and showering phase, one must carefully remove such events to prevent double-counting and to keep the Alpgen cross sections for $V+b \bar{b} / c \bar{c}$ events consistent. Single top MC samples use COMPHEP [50, 51, 52].

Pythia does not model tau or $b$ decays well. Two custom packages, Tauola[53] and Evtgen[54], handle tau and $b$-flavored particle decays, respectively. 


\subsection{Detector simulation and reconstruction}

Generated events then pass through the detector and electronics simulation before being reconstructed using the same software as the data.

\subsection{1 d0gstar}

d0gstar stands for D $\varnothing$ GEANT Simulation of Total Apparatus Response. It is a GEANT-based[55] simulation that models the generator particle interactions in the detector, namely energy loss, showering, energy and charge deposition, and multiple scattering.

\section{$5.2 .2 \mathrm{~d} 0 \operatorname{sim}$}

D0sim is the electronics simulation. It takes the d0gstar output as its input and models the D $\varnothing$ electronics response, adding in electronic noise, inefficiency, and pile-up from minimum bias events. Minimum bias events are low-energy interactions that occur in addition to the main hard scatter in the event; these also contribute to the energy deposition and signal in the detector. The minbias events are taken from collider data using dedicated minimum and zero bias triggers. The d0sim output is in the same format as the detector data, allowing reconstruction with the same software.

\subsubsection{Reconstruction}

Reco is used to reconstruct the MC events just as in data.

\subsection{Simulation corrections}

Due to imperfect modeling, selection efficiencies will often be different in data and Monte Carlo. To achieve good data/MC agreement we must correct for these differences. We apply corrections on muons, taus, and jets, and on such quantities as instantaneous luminosity, beam position, and $Z$ boson $p_{T}$.

\subsubsection{Muon ID Corrections}

There are four chief corrections made to MC muons so as to make the $\mathrm{MC}$ better match the data [44]. They are:

- Quality correction corrections 
- Track match efficiency corrections

- Isolation efficiency corrections

- $\Delta R$ efficiency corrections

The Quality correction measurement uses a tag-and-probe method on $Z \rightarrow$ $\mu \mu$ events similar to the trigger efficiency measurement. The correction is the ratio of the efficiency of the probe muon to pass the set of quality criteria in data and $\mathrm{MC}$, typically parametrized in muon $\eta_{\text {det }}$ and $\phi$. During RunIIa the average Data/MC ratio for loose muons with a medium track match was 0.995. [44]

The Track match efficiency correction also uses a tag-and-probe method. Since the track match efficiency is naturally related to the tracking system, the data/MC ratio is parametrized in track $\eta_{\text {det }}$, due to the CFT, and in $z$, due to difference in SMT hit efficiency in the interaction region (also, the SMT has different construction in different $z$ regions, as discussed in sec. 2.2.2). In RunIIa the average Data/MC ratio for a medium track math was 0.93 .

The Isolation efficiency correction also uses tag-and-probe, but with additional constraints on the tag muon: it must also pass a loose isolation to reject heavy-flavor contamination (i.e. muons coming from a semileptonic $b$ decay), and the tag-probe invariant mass must be consistent with the $Z$ mass. While the isolation efficiency does depend on luminosity, the dependence is well-modeled in the Monte Carlo, and discrepancies only appear at the highest instantaneous luminosities, which comprise a small portion of the total dataset.

\subsubsection{Tau-ID corrections}

As there is currently no tau-specific energy scale at DØ, we employ a mechanism to correct the tau energy in $\mathrm{MC}$ so that the MC energy spectrum agrees better with the data [56]. It applies a correction to the tau energy as a linear function of the EM fraction of type 1 and 2 taus:

$$
E_{\text {Tcorr }}=E_{T}(A \times e m f-B)
$$

For type 3 taus the correction is a linear function of the sum of the track momenta:

$$
E_{T c o r r}=E_{T}\left(A \times \sum_{t r k} p_{T(t r k)}-B\right)
$$


in both equations $A$ and $B$ are tau type-specific constants determined in [56]. Table 5.1 lists the constants for each tau type.

\begin{tabular}{|c|c|c|}
\hline Tau Type & $A$ & $B$ \\
\hline \hline Type 1 & -0.131 & 1.162 \\
Type 2 & -0.298 & 1.371 \\
Type 3 & -0.003 & 1.234 \\
\hline
\end{tabular}

Table 5.1: constants in equations 5.1 and 5.2 for each tau type.

\subsubsection{Jet Corrections}

Corrections to jet energies consist chiefly of jet shifting, smearing, and removal (JSSR) corrections. Jet momenta are smeared (i.e. multiplied by a factor randomly sampled from a normal distribution so that the resulting jet momentum resolution in $\mathrm{MC}$ is the same as in data. Additionally, the jet reconstruction efficiency is slightly higher in MC. To account for this we generate a random number for each jet according to a uniform distribution; if the random number is outside of the allowed range (which is 1 minus the data/MC efficiency difference) we remove the jet. The appropriate scale and smearing factors are in [57].

\subsubsection{Luminosity Reweighting}

d0sim overlays collider data minimum bias events on the MC to simulate underlying additional interactions that occur in the detector. However, the instantaneous luminosity distribution, or luminosity profile, of the minimum bias overlay sample is static, while the instantaneous luminosity profile of the data sample changes over time. Thus there comes a point at which the min bias overlay no longer accurately reflects conditions in the data. The solution is to re-weight the $\mathrm{MC}$ events so their the luminosity profile of each MC sample matches that of the data. This is done in practice by measuring the luminosity profile of the data sample, simply by plotting the instantaneous luminosity of each data event, and re-normalizing the resulting distribution so that the integral of the distribution is 1 . Then, one does the same thing to the MC sample. One can then compute the ratio of data to $\mathrm{MC}$ for the instantaneous luminosity value in each $\mathrm{MC}$ event; this ratio is the weight given to the MC event. If one does this for all MC events then the luminosity profile will be transformed into that of the data. 


\subsubsection{Beam position Reweighting}

Vertex finding and reconstruction efficiencies depend on the $z$ position of the vertex; the farther from the center of the detector, the lower the efficiency. We reweight the primary vertex $z$ distribution in the MC samples to match the data; this effectively corrects for efficiency effects as one moves out in $z$ [58],[59]. The first step is to fit the primary vertex $z$ distribution in zero bias events (so as not to bias the measurement with any particular process) to a convolution of two Gaussian beams, and the beam beta function

$$
\begin{aligned}
\frac{d \mathcal{L}(z)}{d z} & =N_{p} N_{\bar{p}} \frac{1}{\sqrt{2 \pi \sigma z}} \frac{e^{\frac{-(z-z 0 z)^{2}}{2 \sigma^{2}}}}{z 4 \pi \sigma_{x}(z) \sigma_{y}(z)} \\
\sigma_{T}^{2}(z) & =\frac{1}{6 \pi \gamma} \epsilon_{T} \beta_{T}^{*}\left(1+\frac{\left(z-z_{0 T}\right)^{2}}{\beta_{T}^{*}}\right)
\end{aligned}
$$

where one obtains the parameters according to [59] for several different data-taking periods within RunIIb. Then we take 5-10 GeV di-jet and $\gamma+$ jet Monte Carlo and reweight the production vertex distribution to be that of the data (the di-jet $\mathrm{MC}$ behaves roughly as zero bias data at low $p_{T}$, such as 5$10 \mathrm{GeV}$.) Finally, we reweight the reconstructed vertex distribution according to the production distribution, and parametrizes the ratio as a function of vertex $z$ for several different instantaneous luminosity bins; the ratio is then a weight applied to the MC. The ratios for the individual data-taking periods are weighted according to the integrated luminosity in each period to arrive at the final ratio.

\subsection{6 $Z-p_{T}$ Reweighting}

It is known that neither PyTHIA nor AlPGEN reproduces the $p_{T}$ spectrum of the $Z$ boson correctly [60]. A mechanism has been developed [61] to reweight the $Z$ inclusive and $Z+$ jets $\mathrm{MC}$ as a function of the true $Z p_{T}$ at MC-generator level so that the MC describes the data. $Z \rightarrow e e$ events are used for the re-weighting derivation because a very pure data sample with good $p_{T}$ resolution can be obtained and directly compared with the MC. We re-weight both the $Z \rightarrow \mu \mu$ and $Z \rightarrow \tau \tau$ samples using the results derived from the $Z \rightarrow e e$ sample. Events with one or more jets in addition to the $Z$ have different $Z p_{T}$ spectra than those events without additional jets, so an additional correction that is jet multiplicity-dependent [62] is applied on top of the correction derived for the inclusive jet case. When determining an event's jet multiplicity we first remove jets that overlap with the $\tau$ candidate. 


\section{Chapter 6}

\section{$\phi b$ Analysis Methodology}

In this chapter we describe the analysis procedures and selections in detail. We discuss the dataset, MC samples, selection cuts, multijet background estimation, multivariate techniques to separate signal and background, and present the event yields.

\subsection{Dataset and Monte Carlo Samples}

\subsubsection{Data}

We use the MU inclusive dataset, defined as all events with at least one loose muon having $p_{T}>8 \mathrm{GeV}$. We apply the data quality cuts to remove runs where any of the calorimeter, CFT, CTT, SMT, and Muon systems were flagged as having problems. We divide the dataset into five periods corresponding to the trigger versions used: the first period covers versions from v15.00 to v15.13, the second is from v15.14 to v15.19, the third is from v15.20 to v15.53, fourth is from v15.60 to v15.90, and the last is v15.90 to v16.00. Table 6.1 contains the integrated luminosity for each period and the trigger used to calculate the luminosity, which is $1.6 \mathrm{fb}^{-1}$ in total. We require that the event fires at least one of the nine available single muon triggers or one of twenty-four muon + tau triggers (sec. 3.2).

\subsubsection{Monte Carlo}

Table 6.2 lists the background Monte Carlo (MC) samples used in the analysis. We use Pythia to generate the $Z \rightarrow e e$ and di-boson Monte Carlo. The $t \bar{t}$, vector boson $(W, Z)+$ jets and $(W, Z)+$ heavy flavor $(c \bar{c}, b \bar{b})+$ jets Monte Carlo samples were generated with ALPGEN, using PYTHIA for hadronization and 


\begin{tabular}{|c|c|c|}
\hline Trigger List Version & $\int \mathcal{L} d t\left(\mathrm{pb}^{-1}\right)$ & Luminosity Reference trigger \\
\hline $15.00-15.13$ & 167.11 & MUHI2_TK12_TLM12 \\
\hline $15.14-15.19$ & 41.33 & MUHI2_TK12_TLM12 \\
\hline $15.2-15.53$ & 554.52 & MUHI2_TK12_TLM12 \\
\hline $15.60-15.90$ & 451.59 & MUHI2_TK12_TLM12 \\
\hline $15.90-16.00$ & 396.32 & MUHI2_TK12_TLM12 \\
\hline Total Lumi & 1610.87 & MUHI2_TK12_TLM12 \\
\hline
\end{tabular}

Table 6.1: Breakdown of dataset integrated luminosity by period. The reference trigger used for the luminosity calculation is also given. For the analysis selection, we require the event to fire one of the single muon triggers used in the standard "OR" trigger suite or one of 24 muon+tau triggers.

showering. The $n$-lp samples taken together for each $(W, Z)$ process comprise the complete sample for that process. The signal Monte Carlo is the SM $H b \rightarrow \tau_{\mu} \tau_{h} b+X$ process generated using PYTHIA for mass points from 90 to $160 \mathrm{GeV}$ with details given in Table 6.3. It is acceptable to use the SM process to model our signal because in this mass range the neutral Higgs Bosons in SUSY are nearly degenerate in mass and have extremely similar detector signatures to the SM Higgs. In all MC samples we reject events containing a zero bias overlay event from a bad luminosity block as is done with all collider data. We normalize the remaining events using the theoretical cross section for the process, the acceptance times selection efficiency, and the luminosity of the data sample. We also apply all weights and correction from Chapter 5. The cross sections are shown in tables 6.2 and 6.3. The listed cross sections for the ALPGEN processes are Leading Log (LL), and thus require an additional scaling factor ( $K$-factor) to bring them up to their NNLO values. In $Z+$ jet events this factor is 1.34 ; for $W+$ jets it is 1.23 . For the $W+$ heavy flavor samples, we apply an additional $K$-factor of 1.9 on top of the 1.23 factor. For the $Z b \bar{b}+$ jets samples we apply an additional $k$-factor of 1.96 on top of the 1.34; the 1.96 is the product of a theoretical contribution of 1.52 and an empirical correction of $1.3[63]$. To the $Z c \bar{c}+$ jets samples we apply an additional $k$-factor of $2.15 ; 1.67$ for theory and a 1.3 empirical correction[63]. The $t \bar{t} \rightarrow$ di-lepton samples receive a $1.32 \mathrm{~K}$-factor, while we apply a factor of 1.41 to the $t \bar{t} \rightarrow$ lepton + jet samples. 
Table 6.2: Monte Carlo background samples used in the analysis with the number of generated events after data quality selection, and cross section, and K-factor. For the $W, Z+$ jets and $t \bar{t}$ samples, "excl" means exclusive, or exactly that many additional partons were generated. "Incl" means inclusive, meaning that many or greater.

\begin{tabular}{|c|c|c|c|}
\hline Process & $\mathrm{N}$ events & $\sigma \times B R(\mathrm{pb})$ & K-factor \\
\hline$Z+0 l p \rightarrow \tau \tau+0 \operatorname{lp}$ excl & 971,719 & 132.74 & 1.34 \\
\hline$Z+1 l p \rightarrow \tau \tau+1 \mathrm{lp} \mathrm{excl}$ & 567,702 & 40.57 & 1.34 \\
\hline$Z+2 l p \rightarrow \tau \tau+2 \mathrm{lp} \mathrm{excl}$ & 271,832 & 9.75 & 1.34 \\
\hline$Z+3 l p \rightarrow \tau \tau+3$ lp incl & 172,030 & 3.12 & 1.34 \\
\hline$Z c \bar{c}+0 l p \rightarrow \tau \tau c \bar{c}+0 \mathrm{lp}$ excl & 260,691 & 0.93 & 2.15 \\
\hline$Z c \bar{c}+1 l p \rightarrow \tau \tau c \bar{c}+1 \mathrm{lp}$ excl & 101,099 & 0.50 & 2.15 \\
\hline$Z c \bar{c}+2 l p \rightarrow \tau \tau c \bar{c}+2 \mathrm{lp}$ incl & 50,843 & 0.28 & 2.15 \\
\hline$Z b \bar{b}+0 l p \rightarrow \tau \tau b \bar{b}+0 \mathrm{lp}$ excl & 193,125 & 0.42 & 1.96 \\
\hline$Z b \bar{b}+1 l p \rightarrow \tau \tau b \bar{b}+1 \mathrm{lp} \mathrm{excl}$ & 98,305 & 0.19 & 1.96 \\
\hline$Z b \bar{b}+2 l p \rightarrow \tau \tau b \bar{b}+2 \mathrm{lp}$ incl & 43,900 & 0.10 & 1.96 \\
\hline$Z+0 l p \rightarrow \tau \tau+01 p$ excl $\mathrm{m}=130-250$ & 373,526 & 0.88 & 1.34 \\
\hline$Z+1 l p \rightarrow \tau \tau+11 \mathrm{lp}$ excl $\mathrm{m}=130-250$ & 176,494 & 0.36 & 1.34 \\
\hline$Z+2 l p \rightarrow \tau \tau+2 \mathrm{lp}$ excl $\mathrm{m}=130-250$ & 168,179 & 0.09 & 1.34 \\
\hline$Z+3 l p \rightarrow \tau \tau+3$ lp incl $\mathrm{m}=130-250$ & 156,581 & 0.03 & 1.34 \\
\hline$Z c \bar{c}+0 l p \rightarrow \tau \tau c \bar{c}+0 \mathrm{lp}$ excl $\mathrm{m}=130-250$ & 92,369 & 0.007 & 1.34 \\
\hline$Z c \bar{c}+1 l p \rightarrow \tau \tau c \bar{c}+1 \mathrm{lp}$ excl $\mathrm{m}=130-250$ & 50,457 & 0.004 & 1.34 \\
\hline$Z c \bar{c}+2 l p \rightarrow \tau \tau c \bar{c}+2 \mathrm{lp}$ incl $\mathrm{m}=130-250$ & 46,631 & 0.002 & 1.34 \\
\hline$Z b \bar{b}+0 l p \rightarrow \tau \tau b \bar{b}+0 \mathrm{lp}$ excl $\mathrm{m}=130-250$ & 88,410 & 0.003 & 1.34 \\
\hline$Z b \bar{b}+1 l p \rightarrow \tau \tau b \bar{b}+1 \mathrm{lp}$ excl $\mathrm{m}=130-250$ & 45,681 & 0.002 & 1.34 \\
\hline$Z b \bar{b}+2 l p \rightarrow \tau \tau b \bar{b}+2 \mathrm{lp}$ incl $\mathrm{m}=130-250$ & 41,477 & 0.001 & 1.34 \\
\hline$Z+0 l p \rightarrow \tau \tau+0$ lp excl $\mathrm{m}=15-75$ & 765,612 & 337.47 & 1.34 \\
\hline$Z+1 l p \rightarrow \tau \tau+1 \mathrm{lp}$ excl $\mathrm{m}=15-75$ & 516,977 & 40.029 & 1.34 \\
\hline$Z+2 l p \rightarrow \tau \tau+2 \mathrm{lp}$ excl $\mathrm{m}=15-75$ & 285,625 & 9.65 & 1.34 \\
\hline$Z+3 l p \rightarrow \tau \tau+3 \operatorname{lp}$ incl $\mathrm{m}=15-75$ & 278,019 & 2.65 & 1.34 \\
\hline$Z+0 l p \rightarrow \mu \mu+0 \mathrm{lp}$ excl & $1,072,983$ & 133.04 & 1.34 \\
\hline$Z+1 l p \rightarrow \mu \mu+1 \mathrm{lp}$ excl & 609,897 & 40.74 & 1.34 \\
\hline$Z+2 l p \rightarrow \mu \mu+2 \mathrm{lp} \mathrm{excl}$ & 406,615 & 9.76 & 1.34 \\
\hline$Z+3 l p \rightarrow \mu \mu+3 \operatorname{lp}$ incl & 150,323 & 3.11 & 1.34 \\
\hline$Z c \bar{c}+0 l p \rightarrow \mu \mu c \bar{c}+0 \mathrm{lp}$ excl & 194,366 & 0.93 & 2.15 \\
\hline$Z c \bar{c}+1 l p \rightarrow \mu \mu c \bar{c}+11 p$ excl & 96,476 & 0.50 & 2.15 \\
\hline$Z c \bar{c}+2 l p \rightarrow \mu \mu c \bar{c}+2 \mathrm{lp}$ incl & 51,637 & 0.28 & 2.15 \\
\hline
\end{tabular}


Table 6.2 - continued from previous page

\begin{tabular}{|c|c|c|c|}
\hline Process & $\mathrm{N}$ events & $\sigma \times B R(\mathrm{pb})$ & K-factor \\
\hline$Z b b+0 l p \rightarrow \mu \mu b b+0 \operatorname{lp}$ excl & 206,160 & 0.42 & 1.96 \\
\hline$Z b \bar{b}+1 l p \rightarrow \mu \mu b \bar{b}+1 \mathrm{lp}$ excl & 96,476 & 0.19 & 1.96 \\
\hline$Z b \bar{b}+2 l p \rightarrow \mu \mu b \bar{b}+2 \operatorname{lp}$ incl & 44,984 & 0.10 & 1.96 \\
\hline$Z+0 l p \rightarrow \mu \mu+0 \operatorname{lp}$ excl $\mathrm{m}=130-250$ & 364,603 & 0.91 & 1.34 \\
\hline$Z+1 l p \rightarrow \mu \mu+1 \mathrm{lp}$ excl $\mathrm{m}=130-250$ & 178,952 & 0.33 & 1.34 \\
\hline$Z+2 l p \rightarrow \mu \mu+2 \operatorname{lp}$ excl $\mathrm{m}=130-250$ & 165,902 & 0.09 & 1.34 \\
\hline$Z+3 l p \rightarrow \mu \mu+31 p$ incl $m=130-250$ & 159,219 & 0.04 & 1.34 \\
\hline$Z c \bar{c}+0 l p \rightarrow \mu \mu c \bar{c}+01 p$ excl $\mathrm{m}=130-250$ & 79,933 & 0.008 & 1.34 \\
\hline$Z c \bar{c}+1 l p \rightarrow \mu \mu c \bar{c}+11 p$ excl $\mathrm{m}=130-250$ & 48,049 & 0.005 & 1.34 \\
\hline$Z c \bar{c}+2 l p \rightarrow \mu \mu c \bar{c}+2 \operatorname{lp}$ incl $\mathrm{m}=130-250$ & 46,480 & 0.003 & 1.34 \\
\hline$Z b \bar{b}+0 l p \rightarrow \mu \mu b \bar{b}+01 p$ excl $\mathrm{m}=130-250$ & 89,246 & 0.003 & 1.34 \\
\hline$Z b \bar{b}+1 l p \rightarrow \mu \mu b \bar{b}+11 p$ excl $\mathrm{m}=130-250$ & 44,721 & 0.002 & 1.34 \\
\hline$Z b \bar{b}+2 l p \rightarrow \mu \mu b \bar{b}+2 \operatorname{lp}$ incl $\mathrm{m}=130-250$ & 41,976 & 0.001 & 1.34 \\
\hline$Z+0 l p \rightarrow \mu \mu+01 p$ excl $\mathrm{m}=15-75$ & 791,096 & 338.25 & 1.34 \\
\hline$Z+1 l p \rightarrow \mu \mu+1 \mathrm{lp}$ excl $\mathrm{m}=15-75$ & 551,239 & 39.94 & 1.34 \\
\hline$Z+2 l p \rightarrow \mu \mu+21 \mathrm{p}$ excl $\mathrm{m}=15-75$ & 283,271 & 9.66 & 1.34 \\
\hline$Z+3 l p \rightarrow \mu \mu+3 l p$ incl $m=15-75$ & 271,034 & 2.64 & 1.34 \\
\hline$W+0 l p \rightarrow \ell \nu+0 \mathrm{lp}$ excl & $11,245,004$ & 4522.21 & 1.23 \\
\hline$W+1 l p \rightarrow \ell \nu+11 p$ excl & $3,764,422$ & 1279.79 & 1.23 \\
\hline$W+2 l p \rightarrow \ell \nu+2 \operatorname{lp~excl}$ & $1,930,202$ & 298.17 & 1.23 \\
\hline$W+3 l p \rightarrow \ell \nu+3 \operatorname{lp}$ excl & $1,182,576$ & 69.87 & 1.23 \\
\hline$W+4 l p \rightarrow \ell \nu+4 \mathrm{lp}$ excl & $1,123,123$ & 15.72 & 1.23 \\
\hline$W+5 l p \rightarrow \ell \nu+5 \operatorname{lp}$ incl & 580,181 & 4.77 & 1.23 \\
\hline$W c \bar{c}+0 l p \rightarrow \ell \nu c \bar{c}+0 \mathrm{lp}$ excl & 920,965 & 23.99 & 1.9 \\
\hline$W c \bar{c}+1 l p \rightarrow \ell \nu c \bar{c}+1 \mathrm{lp}$ excl & 968,059 & 13.36 & 1.9 \\
\hline$W c \bar{c}+2 l p \rightarrow \ell \nu c \bar{c}+2 \mathrm{lp}$ excl & 576,264 & 5.39 & 1.9 \\
\hline$W c \bar{c}+3 l p \rightarrow \ell \nu c \bar{c}+3$ lp incl & 384,683 & 2.50 & 1.9 \\
\hline$W b \bar{b}+0 l p \rightarrow \ell \nu b \bar{b}+0 l p$ excl & $1,466,935$ & 9.34 & 1.9 \\
\hline$W b \bar{b}+1 l p \rightarrow \ell \nu b \bar{b}+1 \mathrm{lp}$ excl & $1,099,776$ & 4.27 & 1.9 \\
\hline$W b \bar{b}+2 l p \rightarrow \ell \nu b \bar{b}+2 \operatorname{lp}$ excl & 621,952 & 1.53 & 1.9 \\
\hline$W b \bar{b}+3 l p \rightarrow \ell \nu b \bar{b}+3 \operatorname{lp}$ incl & 436,646 & 0.72 & 1.9 \\
\hline$W W \rightarrow$ inclusive & 537,515 & 12.0 & 1.0 \\
\hline$W Z \rightarrow \ell \nu j j$ & 288,625 & 0.84 & 1.0 \\
\hline$W Z \rightarrow j j \ell \ell$ & 280,795 & 0.25 & 1.0 \\
\hline$t \bar{t}+0 l p \rightarrow \ell \nu \ell \nu b \bar{b}+0 \mathrm{lp}$ excl, $\mathrm{m}_{t}=170 \mathrm{GeV}$ & 71,025 & 0.38 & 1.32 \\
\hline$t \bar{t}+0 l p \rightarrow \ell \nu j j b \bar{b}+0 \mathrm{lp}$ excl, $\mathrm{m}_{t}=170 \mathrm{GeV}$ & 719,489 & 1.50 & 1.41 \\
\hline$t \bar{t}+1 l p \rightarrow \ell \nu \ell \nu b \bar{b}+1 \mathrm{lp}$ excl, $\mathrm{m}_{t}=170 \mathrm{GeV}$ & 313,735 & 0.15 & 1.32 \\
\hline
\end{tabular}


Table 6.2 - continued from previous page

\begin{tabular}{|c|c|c|c|}
\hline Process & N events & $\sigma \times B R(\mathrm{pb})$ & K-factor \\
\hline$t \bar{t}+1 l p \rightarrow \ell \nu j j b b+1 \mathrm{lp}$ excl, $\mathrm{m}_{t}=170 \mathrm{GeV}$ & 453,102 & 0.62 & 1.41 \\
$t \bar{t}+2 l p \rightarrow \ell \nu \ell \nu b \bar{b}+2 \mathrm{lp}$ incl, $\mathrm{m}_{t}=170 \mathrm{GeV}$ & 287,342 & 0.08 & 1.32 \\
$t \bar{t}+2 l p \rightarrow \ell \nu j j b \bar{b}+2 \mathrm{lp}$ incl, $\mathrm{m}_{t}=170 \mathrm{GeV}$ & 275,519 & 0.31 & 1.41 \\
\hline
\end{tabular}

\begin{tabular}{|c|c|c|}
\hline Higgs Mass $(\mathrm{GeV})$ & N Events & SM $\sigma \times B R(\mathrm{fb})$ \\
\hline 90 & 83,107 & 0.927 \\
100 & 82,023 & 0.616 \\
110 & 82,227 & 0.432 \\
120 & 82,049 & 0.307 \\
130 & 81,499 & 0.220 \\
140 & 82,042 & 0.162 \\
150 & 81,304 & 0.119 \\
160 & 82,616 & 0.089 \\
\hline
\end{tabular}

Table 6.3: Monte Carlo signal samples used in the analysis with the number of generated events after data quality selection, and SM cross section $\times$ BR in $\mathrm{fb}$ for $\tan \beta=1$. The cross sections come from FEYNHIGGS v2.6.2 [64, 65, $66,67]$, and the branching ratios from HDECAY[68].

\subsection{Event Preselection}

Our final state includes one muon and one hadronic tau. We require that there be one loose muon in the event with $p_{T}>12 \mathrm{GeV},\left|\eta_{\text {det }}\right|<2.0$, and a central track match of medium quality. In addition the muon must be isolated according to the "NP Tight" isolation definition[44], which is $I_{c a l}<2.5 \mathrm{GeV}$ and $I_{t r k}<2.5 \mathrm{GeV}$. The track associated with the muon is constrained to originate from the beam spot (the center of the detector in Monte Carlo) if it does not have any SMT hits. There can be only one muon in the event that passes these requirements; events with more than one are discarded so as to reject $Z \rightarrow \mu \mu$ events and other backgrounds.

We apply the following requirements on the hadronic tau $E_{T}$ and associated track $p_{T}$ :

- Type 1: $E_{T}>10 \mathrm{GeV}, \sum_{t r k} p_{T}>7 \mathrm{GeV}$

- Type 2: $E_{T}>10 \mathrm{GeV}, \sum_{t r k} p_{T}>5 \mathrm{GeV}$ 
- Type 3: $E_{T}>15 \mathrm{GeV}, 1$ track with $p_{T}>5 \mathrm{GeV}, \sum_{\text {trk }} p_{T}>10 \mathrm{GeV}$

To those requirements we additionally demand at least one SMT hit on one of the associated tracks, $R m u>0.5, R m u=\left(1-\frac{c h f}{E_{T \tau}}\right) E_{T \tau} / p_{T t r k}{ }^{1}$ for tau types 1 and 2 , and $E_{T} / p_{\text {Ttrk }}>0.65$ for types 1 and 2 only. We correct the MC tau energy as described in section 5.3.2 and we re-calculate the $N N_{\tau}$ value for $\mathrm{MC}$ event using the corrected energy. We apply the following $N N_{\tau}$ cuts to tau candidates:

- Type 1: $N N>0.9$,

- Type 2: $N N>0.9$,

- Type 3: $N N>0.95$.

Additionally, the tau cannot have any MediumNseg3 muon within $\Delta R<$ 0.5 and must be separated from the selected isolated muon by at least 0.5 in $R$.

We also require that the $\mathrm{W}$ mass variable, $M_{W}=\sqrt{\left(2 E_{T} E_{\mu}^{2} / p_{T \mu}\left(1-\cos \left(\Delta \phi\left(\mu, E_{T}\right)\right)\right)\right)}$ (Figure B.19), be less than 80, 80, and $60 \mathrm{GeV}$ for types 1, 2, and 3, respectively. There must be one primary vertex in the event with $|z|<40 \mathrm{~cm}$, with at least three tracks associated to it.

\subsubsection{Jet Selection}

After muon and tau selection we require at least one good jet in the event, with $\Delta R(\mu$,jet $)>0.5$ and $\Delta R(\tau$, jet $)>0.5$ where $\mu$ is the isolated muon candidate and $\tau$ is a tau candidate that passes the NN requirements. The jet must have $p_{T}>15 \mathrm{GeV},|\eta|<2.5$ and $\left|\eta_{\text {det }}\right|<2.5$, be confirmed in the Level 1 trigger system, be associated with the primary vertex in the event, and pass the standard Jet-ID requirements[69]:

- Electromagnetic fraction (EM) fraction (i.e. the fraction of the energy in the EM section of the calorimeter) $>0.05$

- Electromagnetic fraction $(\mathrm{EM})$ fraction $<0.95$

- Ratio of energy in most energetic cell in the jet to energy in second-most energetic cell in the jet $<10$

- Coarse hadronic fraction $(\mathrm{CH})$ fraction (i.e. the fraction of the energy in the $\mathrm{CH}$ section of the calorimeter $)<0.40$

\footnotetext{
${ }^{1}$ chf is the coarse hadronic fraction, defined as the amount of the tau $E_{T}$ in the coarse hadronic portion of the calorimeter.
} 
- Minimum number of cells containing 90\% of cell energy (n90) $>1$

We correct the (calorimeter) $\mathbb{E}_{T}$ using the $p_{T}$ of good muons, and the energy in the coarse hadronic calorimeter $(\mathrm{CH})$ of each good jet. If there is a jet that passes all cuts but overlaps with a tau, we use this jet, not the tau, in the $\mathbb{E}_{T}$ calculation. Figures 6.1 through 6.3 show several kinematic and topological distributions after the all selection requirements except $b$-tagging. Figures 6.4 through 6.6 show those same distributions after $b$-tagging. Additional kinematic and topological variable distributions are available in Appendix B. An expanded view of the legend used for these plots is available as Figure B.1. Before $b$-tagging the leading backgrounds are $Z \rightarrow \tau \tau$ (red) and QCD multijet events (blue), while after $b$-tagging the leading backgrounds are $t \bar{t}$ (green) and QCD. In all cases the data are the points, and the signal (black line) is not part of the stack, but is shown only for illustrative purposes.
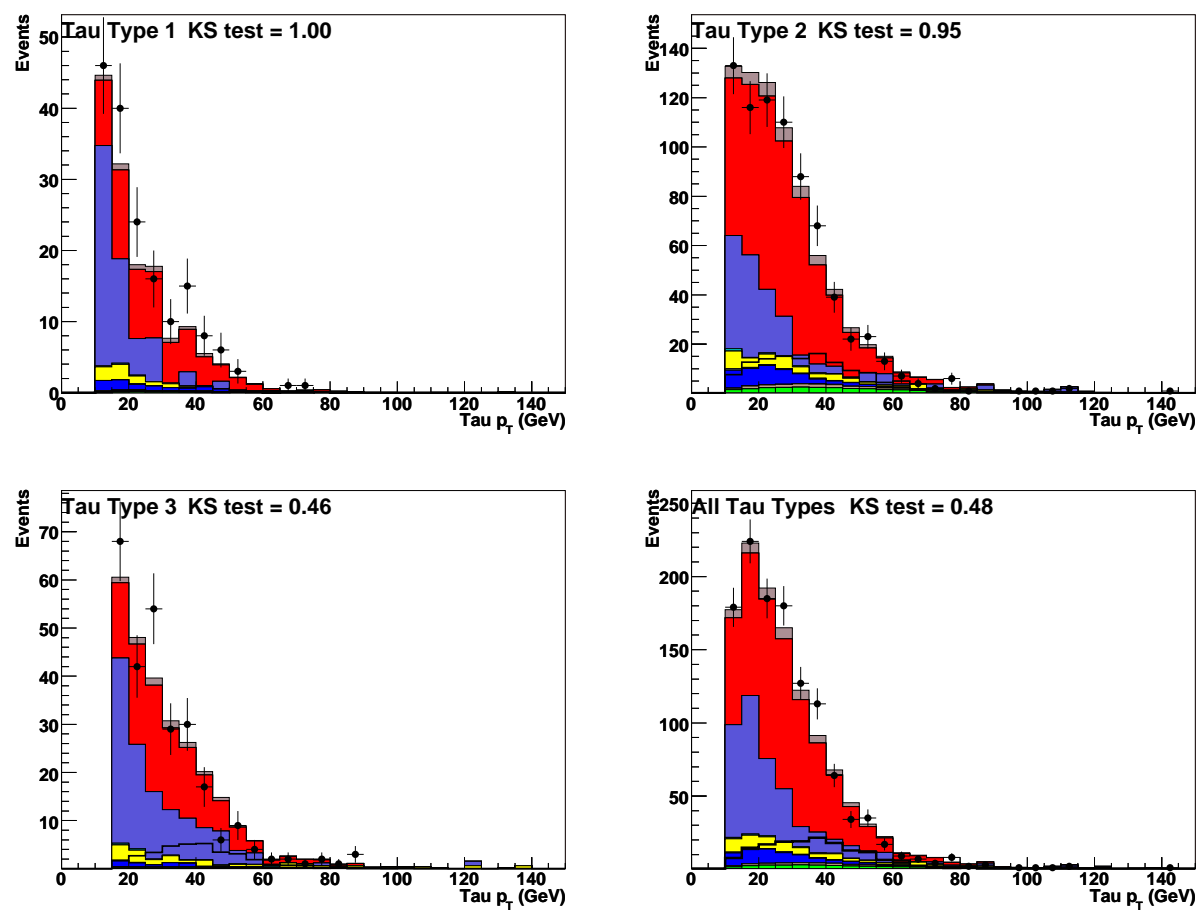

Figure 6.1: Tau $p_{T}$ before $b$-tagging for: Type 1 taus (upper left), Type 2 (upper right), Type 3 (lower left), and all types (lower right) using the Single Muon or $\mathrm{Mu}+\mathrm{Tau} \mathrm{OR}$. 

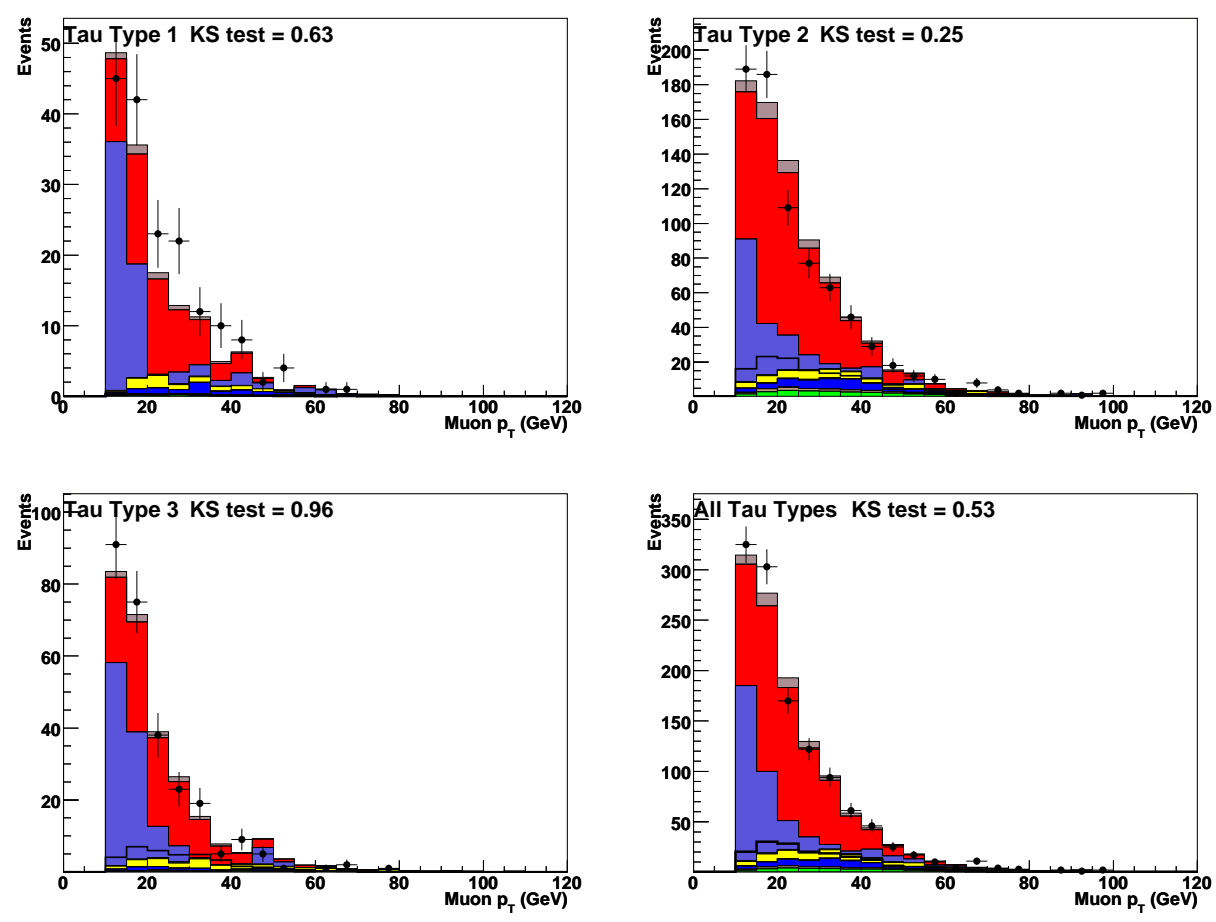

Figure 6.2: Muon $p_{T}$ before $b$-tagging for: Type 1 taus (upper left), Type 2 (upper right), Type 3 (lower left), and all types (lower right) using the Single Muon or $\mathrm{Mu}+\mathrm{Tau}$ OR. 

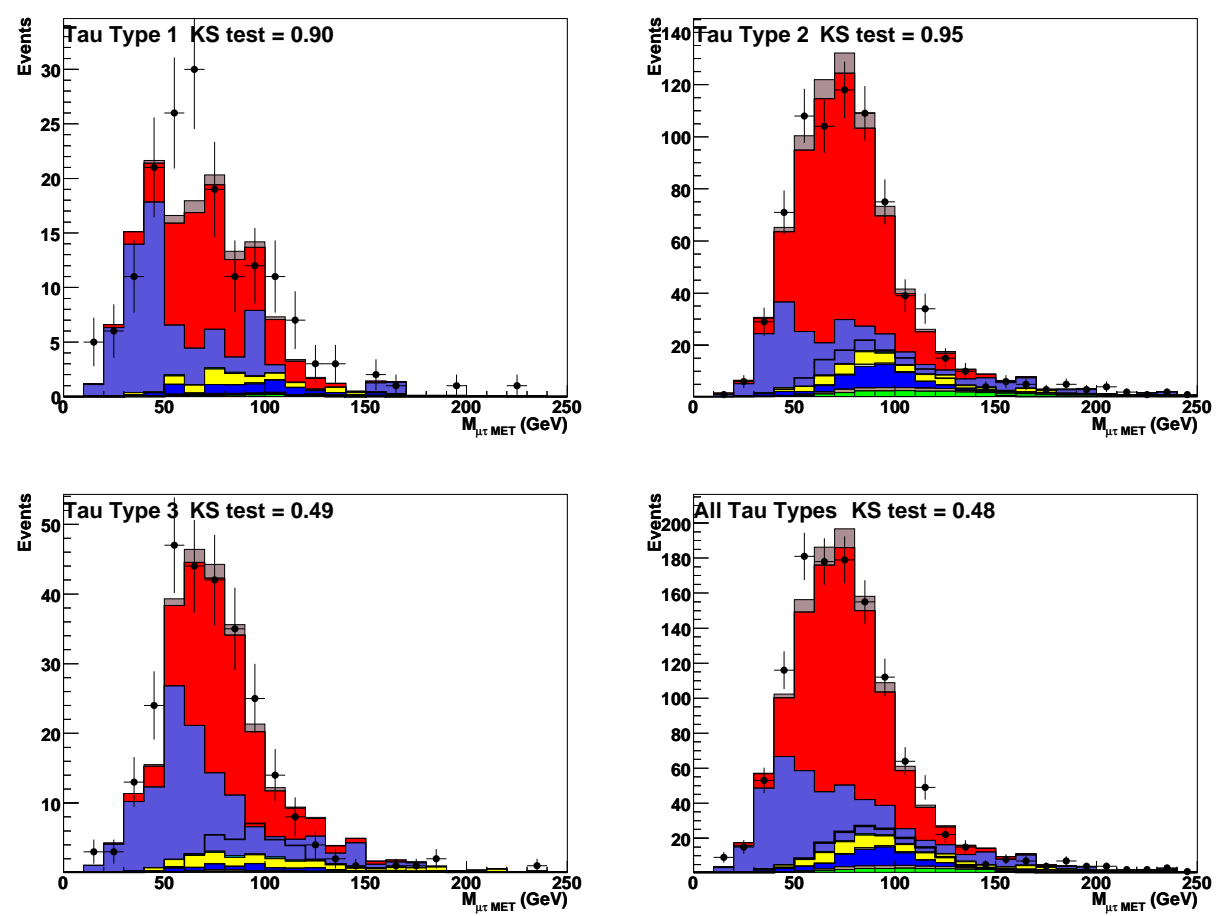

Figure 6.3: Invariant mass of the $\mu, \tau, \mathbb{E}_{T}$ system before $b$-tagging for: Type 1 taus (upper left), Type 2 (upper right), Type 3 (lower left), and all types (lower right). 

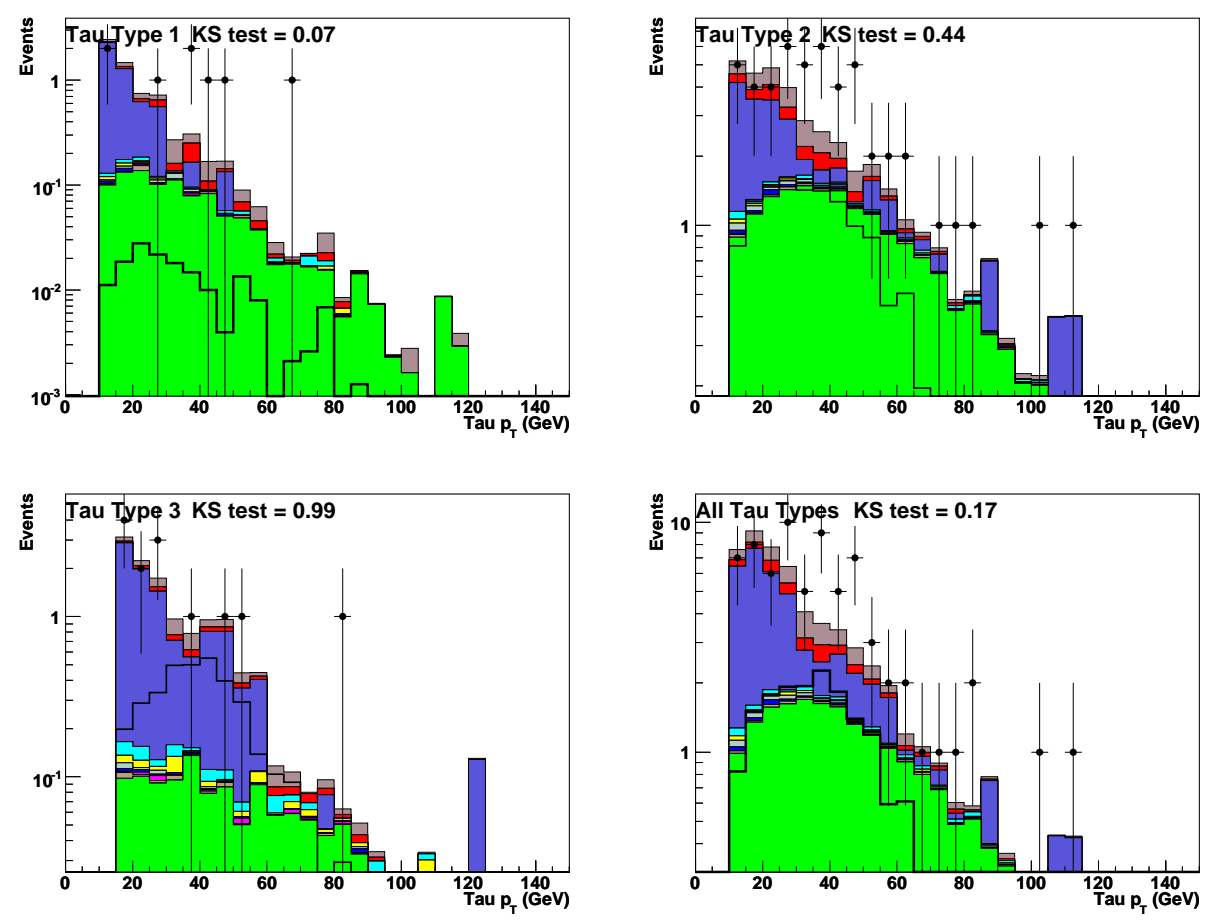

Figure 6.4: Tau $p_{T}$ after $b$-tagging for: Type 1 taus (upper left), Type 2 (upper right), Type 3 (lower left), and all types (lower right) using the Single Muon or $\mathrm{Mu}+\mathrm{Tau} \mathrm{OR}$.

\subsection{QCD Multijet Background Estimation}

We expect both heavy- and light-flavor multi-jet events (QCD) to pass the preselection. Simulations of these events poorly describe the data in the tail region of distributions relevant to this analysis, so the QCD contribution will be estimated from control data samples. To obtain a QCD-enriched sample, we apply the preselection but invert the muon isolation requirement; i.e. $2.5<$ $I_{\text {cal }}<12.0 \mathrm{GeV}$ or $2.5<I_{t r k}<12.0 \mathrm{GeV}$. Additionally we require that the tau has a NN output between 0.3 and 0.9 for all tau types. This reduces the contribution from $Z / \gamma^{*} \rightarrow \tau \tau$ and ensures that the QCD enriched sample will be orthogonal to the signal sample. 

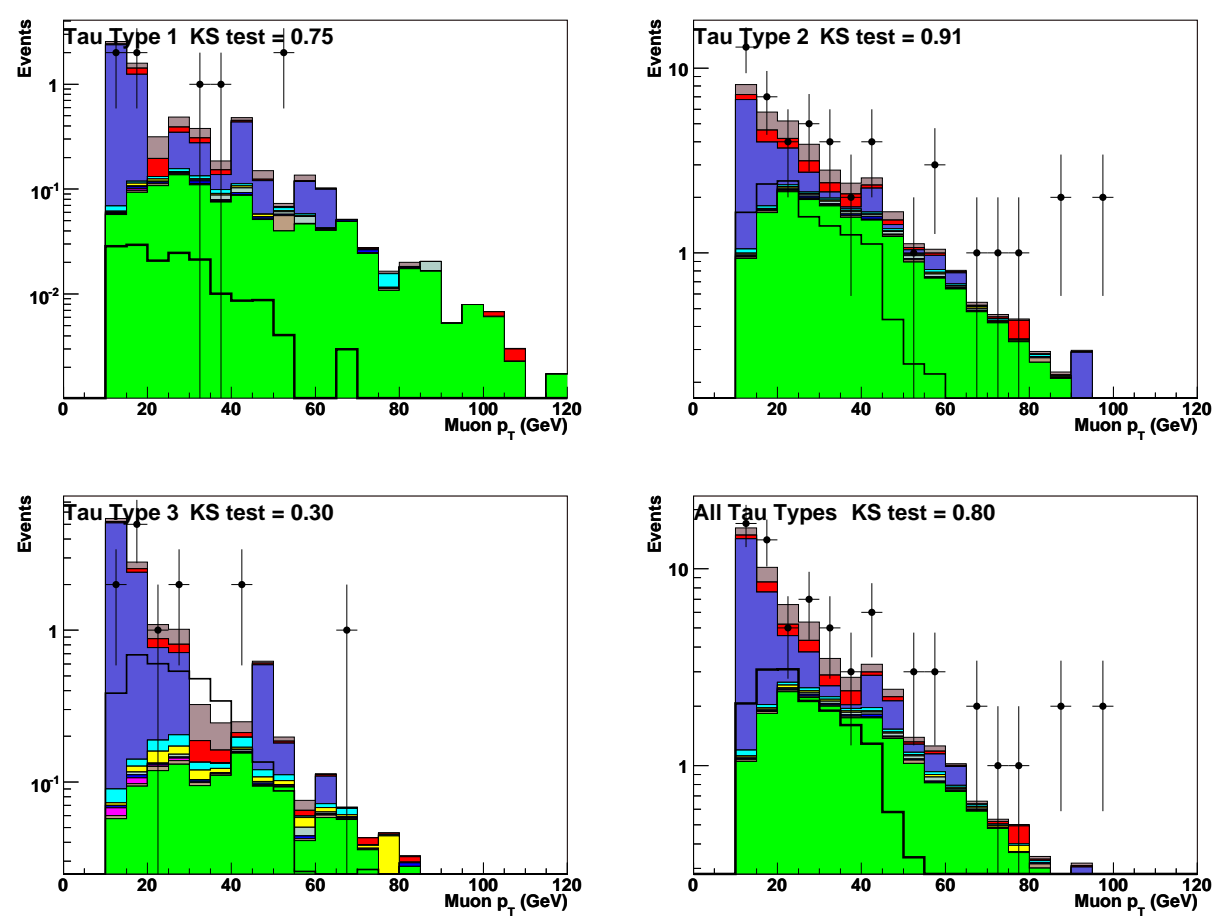

Figure 6.5: Muon $p_{T}$ after $b$-tagging for: Type 1 taus (upper left), Type 2 (upper right), Type 3 (lower left), and all types (lower right) using the Single Muon or $\mathrm{Mu}+\mathrm{Tau} \mathrm{OR}$.

\subsubsection{QCD estimation in pre-btag sample}

QCD events should have an equal number of same-sign (SS) and opposite-sign (OS) events since the signs of the muon and tau in these events should both be random. To determine the number of QCD events in the signal sample before $b$-tagging we take the SS data and subtract the SS MC prediction. The amount of QCD in the signal sample, $N_{Q C D}$, is then

$$
\begin{array}{r}
N_{Q C D}=f_{Q C D} N_{\text {signal }}(S S) \\
f_{Q C D}=\frac{N_{\text {rich }}(O S)}{N_{\text {rich }}(S S)}
\end{array}
$$

where $N_{\text {rich }}(O S)$ and $N_{\text {rich }}(S S)$ are the number of OS and SS events in the QCD enriched sample, respectively. Table 6.4 shows $f_{Q C D}$ for each of the three tau types, while table 6.5 shows the SS data and subtracted MC which produce the final QCD after scaling by $f_{Q C D}$. 

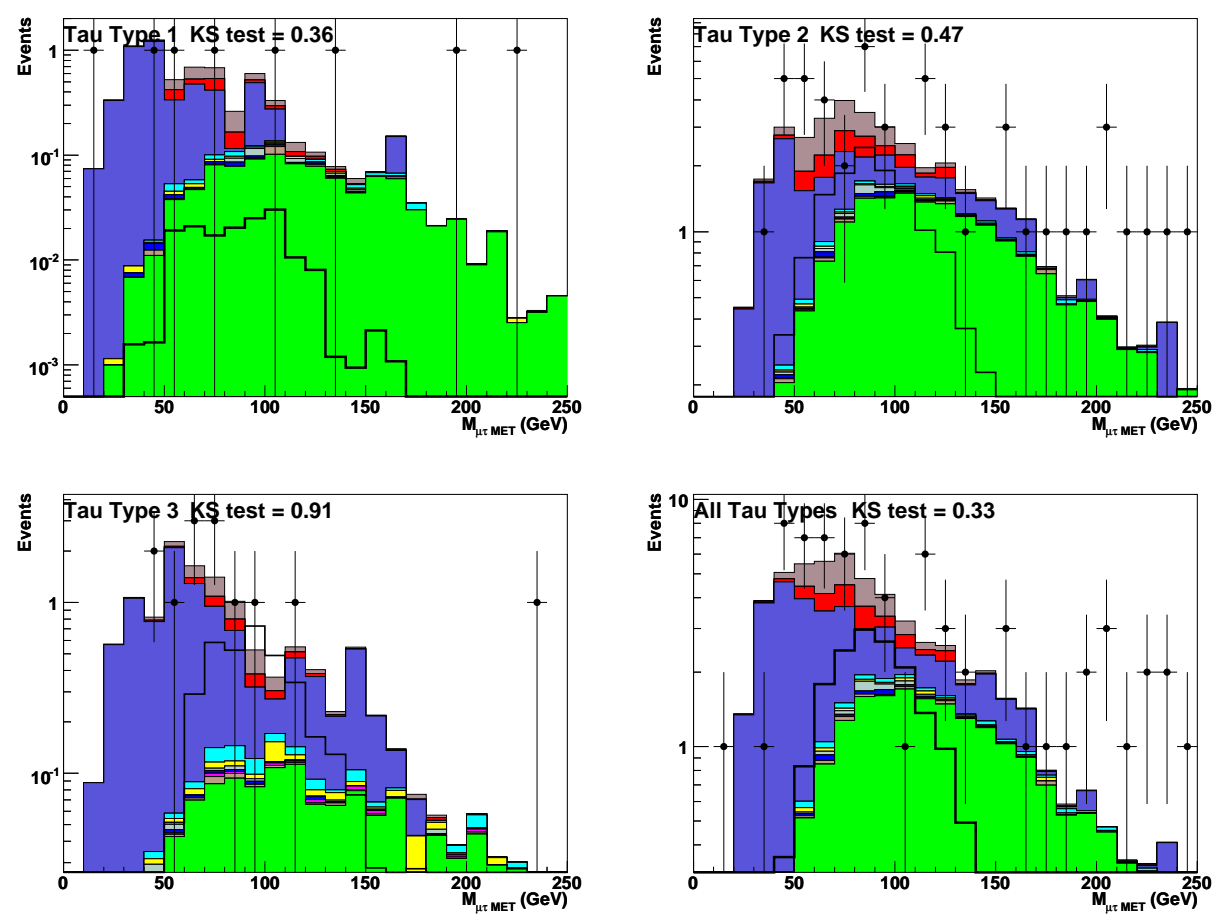

Figure 6.6: Invariant mass of the $\mu, \tau, E_{T}$ system after $b$-tagging for: Type 1 taus (upper left), Type 2 (upper right), Type 3 (lower left), and all types (lower right). 


\begin{tabular}{|c|c|}
\hline$\tau$ Type & $f_{Q C D}$ \\
\hline Type 1 & $1.052 \pm 0.041$ \\
Type 2 & $1.066 \pm 0.027$ \\
Type 3 & $1.039 \pm 0.019$ \\
\hline Average & $1.052 \pm 0.017$ \\
$\left(\chi^{2} /\right.$ dof & $1.6 / 2$ \\
\hline
\end{tabular}

Table 6.4: QCD scaling factors, $N(O S) / N(S S)$ and their statistical errors. We use the type-specific values in the analysis. The average and corresponding $\chi^{2}$ are shown for information only.

\begin{tabular}{|l|c|c|c|}
\hline & Tau Type 1 & Tau Type 2 & Tau Type 3 \\
SS Data & 61 & 171 & 123 \\
SS MC & 4.7 & 24.5 & 18.7 \\
Total QCD & 59.3 & 155.1 & 108.4 \\
\hline
\end{tabular}

Table 6.5: Same-sign (SS) data before b-tagging, along with SS MC (sum of all backgrounds.) We subtract the MC from the data, then multiply the result by the $f_{Q C D}$ factors in table 6.4 to get the final pretag QCD result.

\subsubsection{QCD estimation after $b$-tagging}

The natural extension of the pre-tag QCD method to the final result with a b-tag is simply to require at least one of the jets in the pre-tag QCD sample to be tagged. However, we suffer from poor statistics in this case; we have only 3, 7, and 8 remaining events for tau types 1, 2, and 3, respectively, making it unsuitable for determining the shape of the QCD after $b$-tagging. To determine the QCD contribution after $b$-tagging we develop two largely independent methods and average their results. We use the the direct tagging results above as a cross check to the overall normalization.

\section{TRF based QCD estimation}

In the first method we start from the QCD enriched sample described in Sec. 6.3.1. We look at the jets in the events and compute the " $b$-tagging rate", defined as the fraction of taggable jets that have an NN TIGHT b-tag. Then we parametrize this rate as a function of jet $p_{T}$. We fit the distribution to a hyperbolic tangent function (hereafter called the pseudo TRF). Figure 6.7 shows the $b$-tagging rate as a function of jet $p_{T}$ for the three tau types, with the pseudo TRF overlaid, along with the $\pm 1 \sigma$ contours. Due to the large 
statistical fluctuations within each type, especially at high jet $p_{T}$, we combine all three tau types to reduce fluctuations and improve the uncertainty on the fit. One can see from the right side of figure 6.7 that we do not introduce a significant bias when combining types.

Once we have the pseudo TRF we apply it to the events in the pre-tag (SS data - SS MC) QCD sample as described in Sec. 6.3.1 as a weight in the following way: to get the probability to have at least one b-tagged jet in the event (our requirement in the data), we simply compute $1-\prod_{i}^{\text {Njets }}\left(1-P_{\text {tag }}(i)\right)$. We apply the same pseudo TRF to both data and the same-sign MC. Table 6.8 lists the total QCD for each of the three tau types after b-tagging using this method.
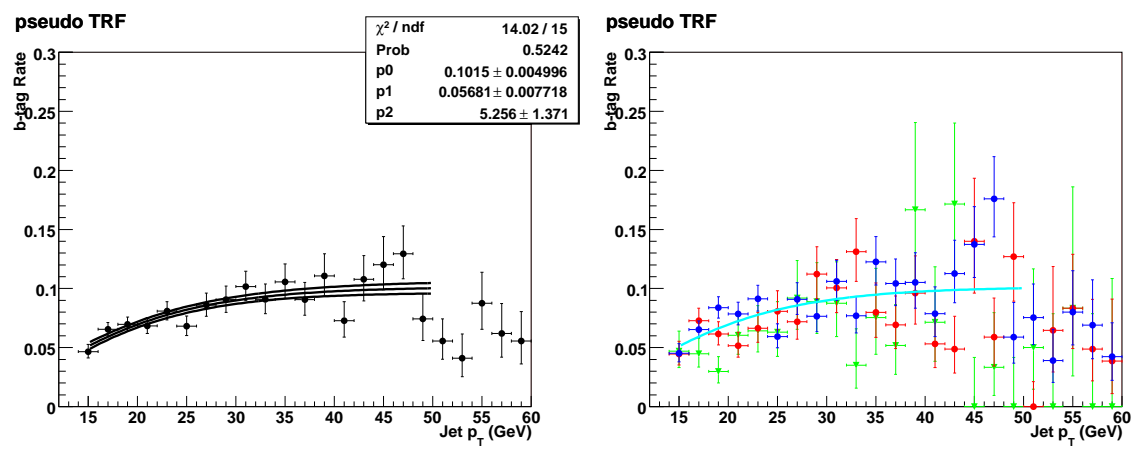

Figure 6.7: $b$-tagging rate as a function of jet $p_{T}$, the pseudo TRF, for all tau types (left) and overlaid over data from the separate tau types(right). Green is Type 1 , red is Type 2, and blue is Type 3 .

\section{Fake-rate based QCD estimation}

In the second method we do require a direct b-tag on a jet in the QCD rich sample described earlier. This gives the number of tagged events with nonisolated muons and bad taus. We then estimate the number of tagged events with isolated muons and good taus by applying ratios of isolated to nonisolated muons and good to bad taus derived from control samples.

To measure the jet $\rightarrow \tau$ fake rate we prepare a sample dominated by $W+$ jets events. We require a medium quality muon with a medium track match and $p_{T}>25 \mathrm{GeV},|\eta|<1.6$, and the same isolation as in the analysis sample. We then require a good jet with $p_{T}>15 \mathrm{GeV}$ to overlap with a tau object with the same kinematic cuts as the analysis, only a loose NN cut of 0.3 . We also require $E_{T}>30 \mathrm{GeV}$ and $50<M_{T}<100 \mathrm{GeV}$, where $M_{T}$ is the transverse 
mass of the muon and $\mathbb{E}_{T}$. We then compute the fraction of these taus that pass our standard $N N_{\tau}$ cuts of $0.9,0.9$, and 0.95 for tau types 1,2 and 3 respectively. We then define the tau fake as

$$
f_{\tau}=\frac{N_{N N_{\tau}>0.9,0.9,0.95}}{N_{0.3<N N_{\tau}<0.9,0.9,0.95}}
$$

Table 6.6 shows the fake rates by tau type.

\begin{tabular}{|c|c|c|}
\hline Type 1 & Type 2 & Type 3 \\
\hline $0.228 \pm 0.037$ & $0.279 \pm 0.025$ & $0.105 \pm 0.009$ \\
\hline
\end{tabular}

Table 6.6: Jet to tau fake rates as a function of tau type and their statistical errors.

To measure the muon isolation fake rate we make another sample based on the QCD enriched sample, but we remove any muon isolation requirement save $I_{\text {cal }}<12.0 \mathrm{GeV}, I_{\text {trk }}<12.0 \mathrm{GeV}$ from the original skimming, both of which are very loose cuts. Thus we have both isolated and anti-isolated muons in this sample. Additionally we require at least one b-tagged jet with $\Delta R>0.5$ from the nearest tau. All taus in the noiso sample must have $0.3<N N_{\tau}<0.9,0.9,0.95$. Events that meet all of these requirements form the "noiso" sample. We subtract the standard model MC contribution with the same cuts, applying the usual $b$-tagging TRFs. Table 6.7 shows the number of data and $\mathrm{MC}$ events per tau type in the noiso sample. To protect against any residual contamination require $\mathbb{E}_{T}<20 \mathrm{GeV}$ in the event when measuring the muon isolation fake rate. We fit the muon isolation rate to a constant for events in the noiso sample with muon $p_{T}$ between 12 and $30 \mathrm{GeV}$ (this results in a negligible loss of QCD events, as can be seen from the pre-tag muon $p_{T}$ plots) and compute a fake rate $f_{\mu}$ of $9.7 \%$, shown in Figure 6.8. We observed no significant difference between tau types so we combine them to arrive at the $9.7 \%$ result. The final number of QCD events in the Fake Rate method is then the jet to tau fake rate and the muon isolation fake rates applied to the noiso sample, with the $\mathbb{E}_{T}$ cut removed, or

$$
N_{Q C D}=f_{\mu} f_{\tau} N_{\text {noiso }}
$$

computed separately for each tau type. Table 6.8 shows the final QCD numbers for the Fake Rate Method. 


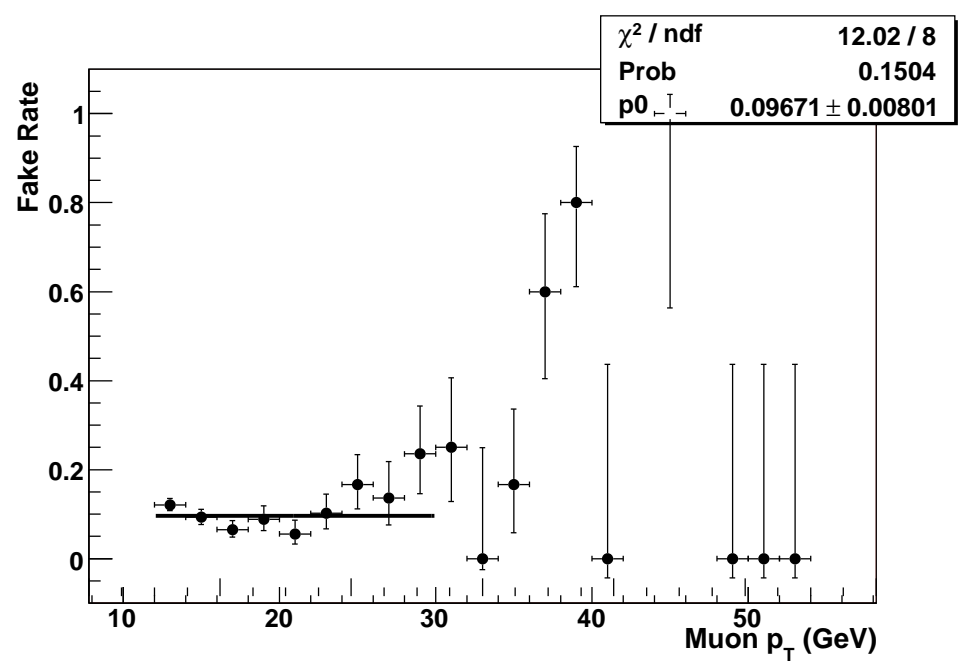

Figure 6.8: Muon isolation rate as a function of muon $p_{T}$ in noiso sample events with $\mathbb{E}_{T}<20 \mathrm{GeV}$.

\begin{tabular}{|c|c|c|c|}
\hline & Tau Type 1 & Tau Type 2 & Tau Type 3 \\
\hline Data & 153 & 421 & 851 \\
Monte Carlo & 3.1 & 20.0 & 39.9 \\
Total & 149.9 & 401.0 & 811.1 \\
\hline
\end{tabular}

Table 6.7: Number of data and background MC events in the noiso sample used in section 6.3.2. We subtract the MC contribution from the data to get the final number for each type.

\subsubsection{Discussion and Final QCD results}

Both the TRF method and Fake Rate method have certain assumptions. In the TRF method one assumes that the muon isolation does not play a role in the sample's flavor composition (which would affect the measured rates) and one is at the mercy of the uncertainty on the pseudo TRF calculation. In the Fake Rate method, one assumes that the $\mathbb{E}_{T}$ does not affect the QCD and flavor composition, but one directly measures the isolation. Thus, the assumptions and weaknesses of one method are not present in the other method, making them complimentary.

To take the final QCD shape we use the TRF method; since we apply it directly to the pre-tag QCD there is a more natural flow from pre-tag to b-tag. Once we take the average of the two methods, separately for each tau type, we re-normalize the TRF Method QCD shape to this average. As an additional cross check we compare the averages to the results of requiring a NN TIGHT 
b-tag on at least one jet in the pre-tag QCD sample. It is very encouraging that the average of the two methods is close to the direct tagging results in all types. For the QCD systematic we propagate the statistical errors on the QCD enriched sample size, the error from the $f_{\mu}$ fit, and the statistical errors on the tau fake rate measurement in table 6.6. Since the spread between the two methods is larger than the statistical uncertainty on the two methods in type 1 , we also take that into account. Our final systematics on the QCD are $25 \%$ for Type 1, $10 \%$ for type 2 , and $10 \%$ for type 3 .

\begin{tabular}{|c|c|c|c|c|}
\hline$\tau$ Type & TRF Method & Fake Rate Method & Average & Direct Tagging Total \\
\hline Type 1 & 5.15 & 3.31 & 4.23 & 3 \\
Type 2 & 11.41 & 10.54 & 10.98 & 7 \\
Type 3 & 8.80 & 8.25 & 8.58 & 8 \\
Total & 25.36 & 22.10 & 23.79 & 18 \\
\hline
\end{tabular}

Table 6.8: Final QCD totals for the different methods of estimation. We take the average of the TRF method and Fake Rate method as the overall normalization, and take the QCD shape from the TRF method.

\subsection{Multivariate Techniques}

After $b$-tagging $t \bar{t}$ and QCD events dominate the background. It is difficult to reduce the QCD or $t \bar{t}$ though additional simple selections without incurring a substantial loss of signal, so we look to multivariate methods to reduce these two backgrounds. We use two separate techniques, one designed to reject $t \bar{t}$ $(\mathrm{KNN})$, and one to reject QCD.

\subsubsection{Top Identification Neural Network (KNN)}

To reject $t \bar{t}$ background we apply a Kinematic Neural Network (KNN) [18]. It is a neural network with four input variables, one hidden layer, and nine neurons, trained on inclusive $t \bar{t}$ PYTHIA MC acting as the background, and signal MC without $b$-tagging acting as signal. Monte Carlo from all mass points is used in the training. The four input variables are the HT, or $E_{T}$ sum of all jets in the event, excluding the tau; the energy of the four-vector sum of the tau candidate, muon candidate, and any jets in the event; the number of jets in the event; and $\Delta \phi(\mu, \tau)$. Figures 6.9 and 6.10 show the input variables for all tau types before and after $b$-tagging, respectively. Figures 6.11 and 6.12 show the KNN output before and after $b$-tagging. A KNN cut of 0.3 offers $\approx 75 \%$ rejection in $t \bar{t}$ with only a $\approx 4 \%$ signal loss. 

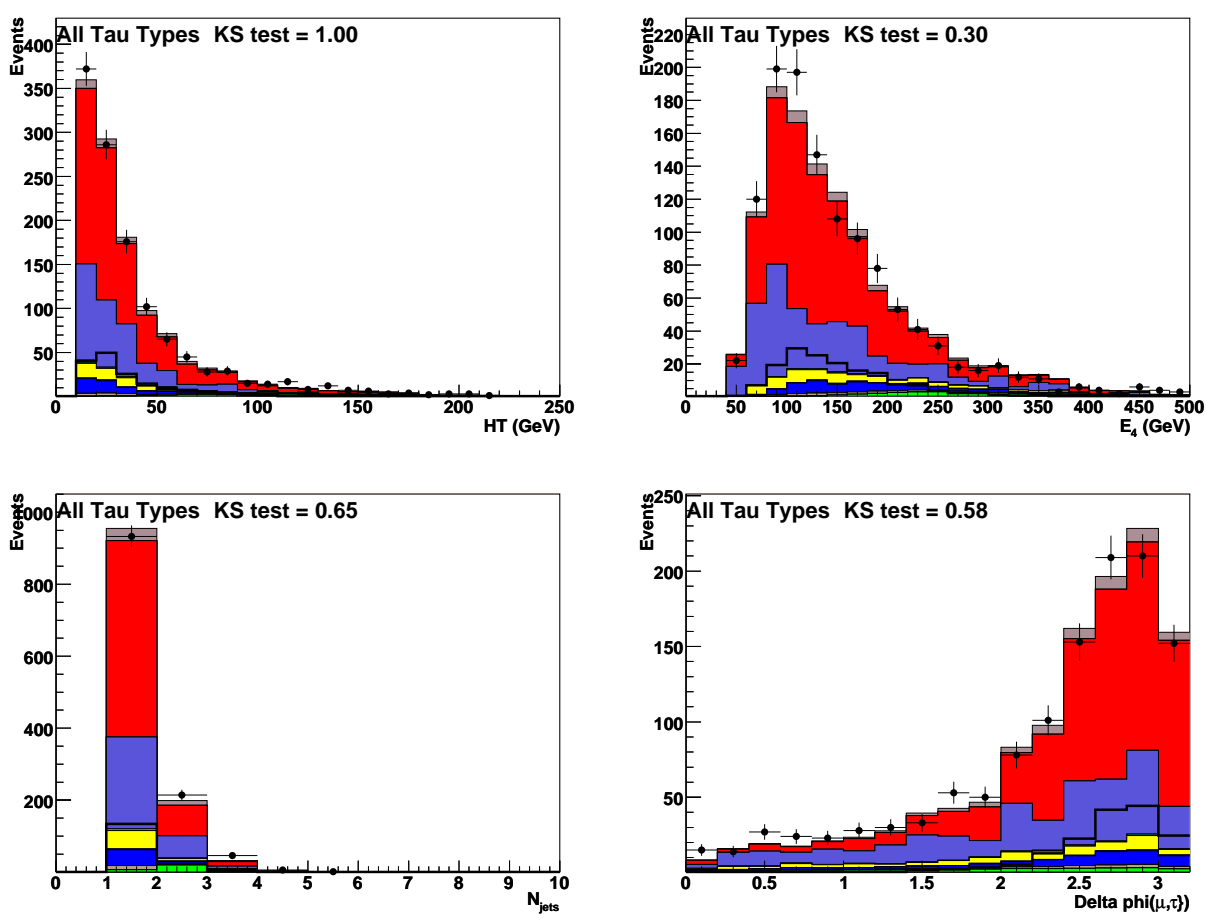

Figure 6.9: KNN Input variables before $b$-tagging for all tau types combined: HT (upper left), Energy from 4 vector sum (upper right), Number of reconstructed jets (lower left), $\Delta \phi(\mu, \tau)$ (lower right).

\subsubsection{QCD Likelihood}

We have insufficient statistics in the pre-tag QCD with which to train a multivariate technique which makes use of variable-to-variable correlations, so to reject $\mathrm{QCD}$ we use a simple unbinned likelihood ratio (hereafter also called LHood), constructed from these five variables:

- muon $p_{T}$

- $\operatorname{tau} p_{T}$

- $d R(\mu, \tau)$

- $\mu-\tau$ invariant mass $\left(M_{\mu \tau}\right)$

- Visible Mass $\left(\mu, \tau, \not_{T}\right)\left(M_{v i s}\right)$

We fit distributions of these variables in the pre-tag QCD and the pretag signal, separately for each signal mass point, to smoothing functions to 

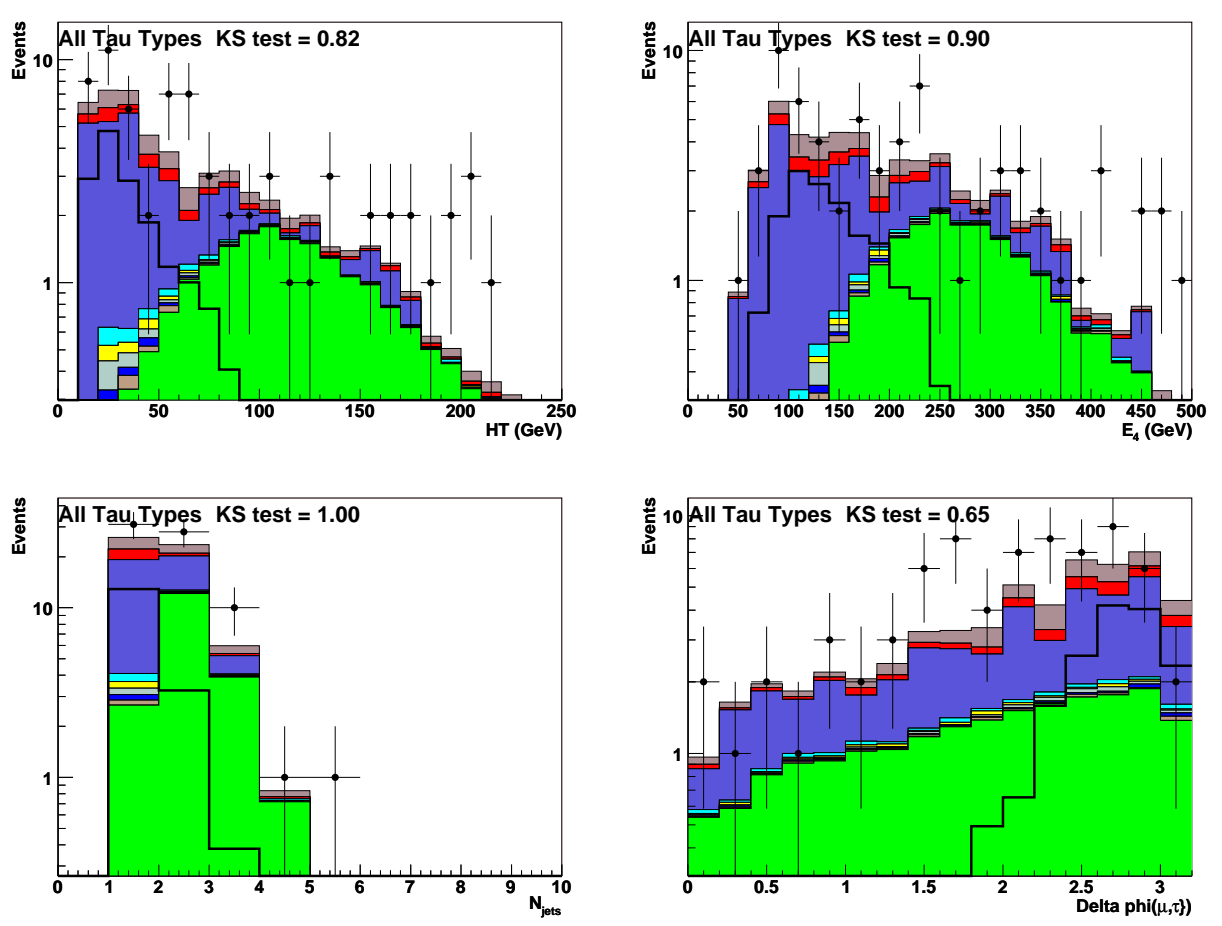

Figure 6.10: KNN Input variables after $b$-tagging for all tau types combined: HT (upper left), Energy from 4 vector sum (upper right), Number of reconstructed jets (lower left), $\Delta \phi(\mu, \tau)$ (lower right).

reduce any statistical fluctuations. Appendix $\mathrm{C}$ contains these distributions and functions. We then define the QCD background $\log$-likelihood $Q$ and the signal $\log$-likelihood $S$ of an event as

$$
\begin{aligned}
\log Q & \equiv \sum_{i} \log \left(\frac{f_{i}^{Q C D}(x)}{\int f_{i}^{Q C D}(x) d x}\right) \\
\log S & \equiv \sum_{i} \log \left(\frac{f_{i}^{\text {Signal }}(x)}{\int f_{i}^{\text {Signal }}(x) d x}\right)
\end{aligned}
$$

in which the sums run over the five input variables. Here $f$ is the smoothing function for a given variable and sample, and the integral serves simply as a normalization factor. 

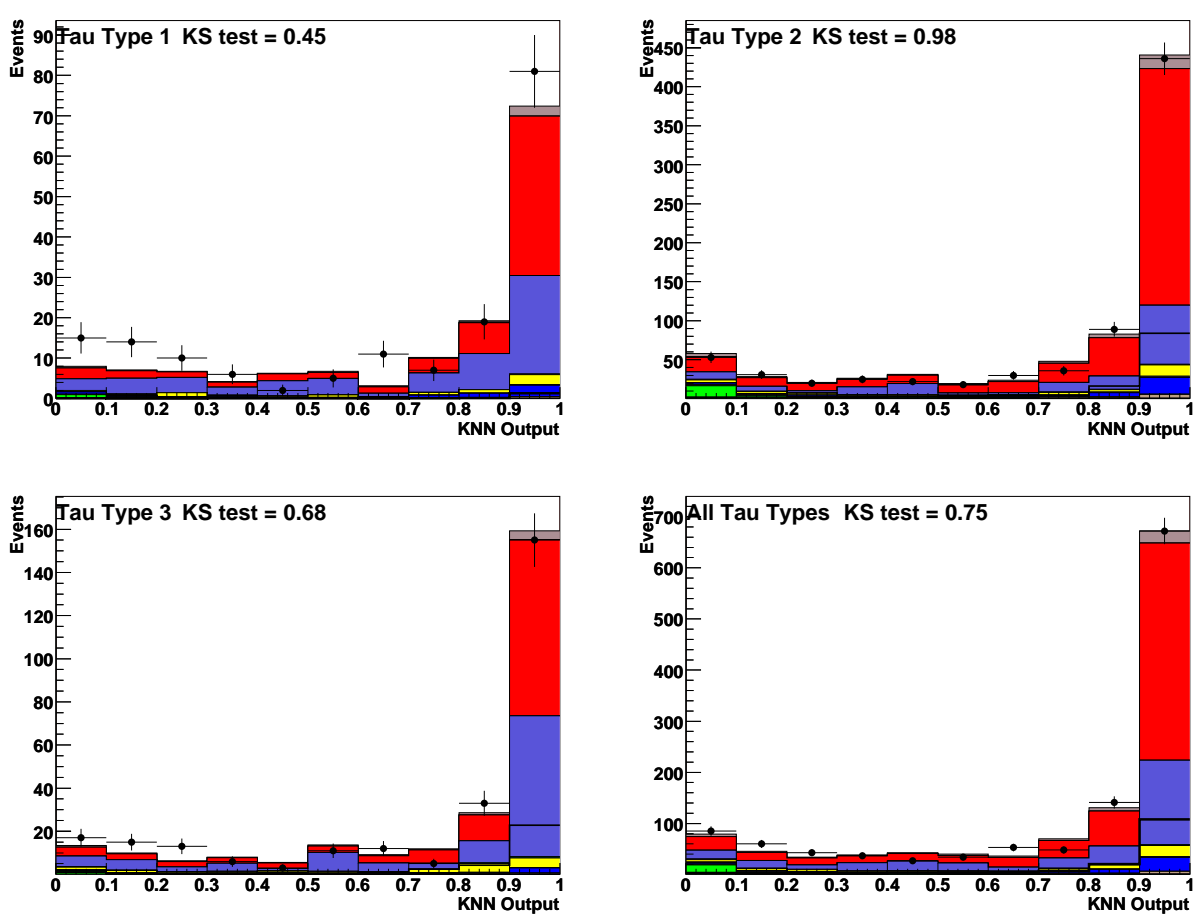

Figure 6.11: KNN output before $b$-tagging for: Type 1 taus (upper left), Type 2 (upper right), Type 3 (lower left), and all types (lower right). 

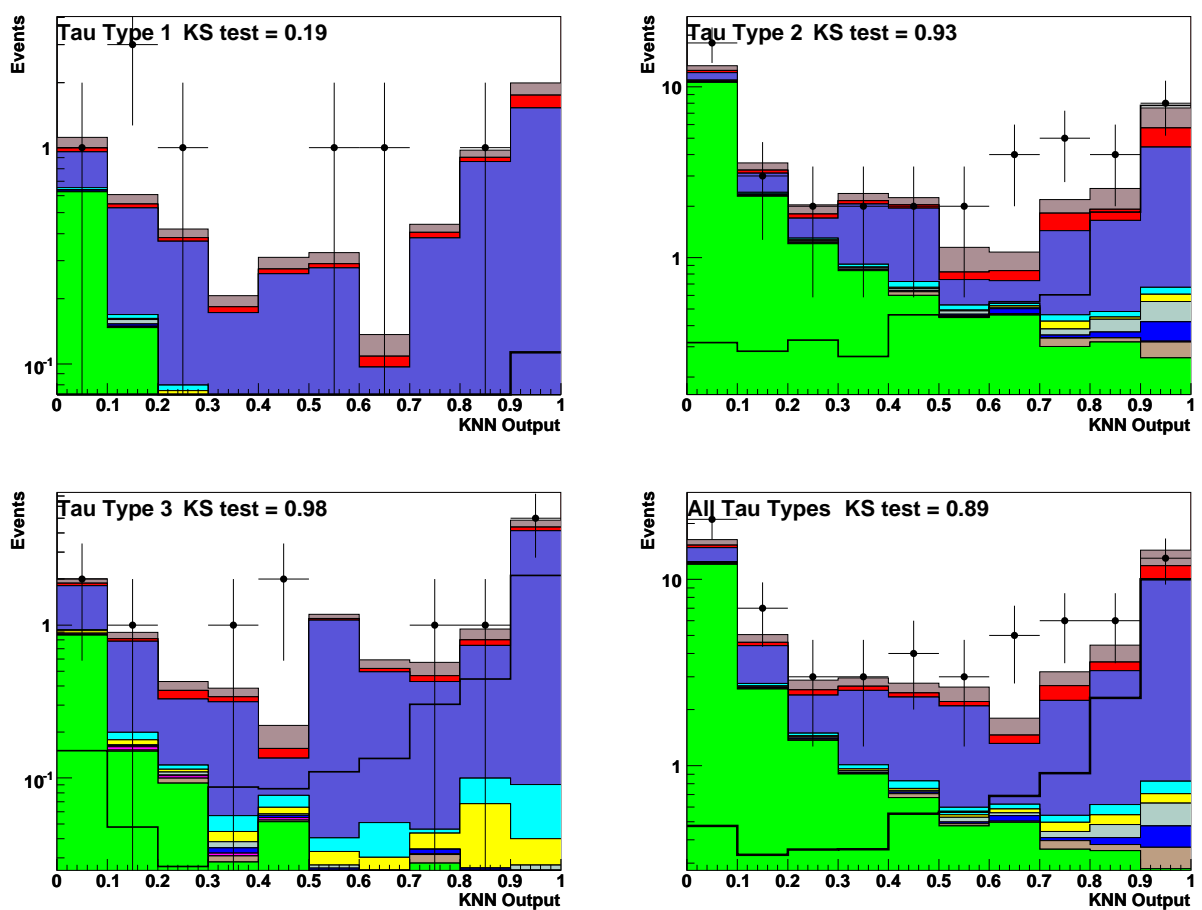

Figure 6.12: KNN output after $b$-tagging for: Type 1 taus (upper left), Type 2 (upper right), Type 3 (lower left), and all types (lower right). 
We can define the likelihood ratio $R=S / Q$ and convert it to the event log likelihood ratio:

$$
\log R \equiv \log S-\log Q
$$

We can easily see that events with a high $R$ (or equivalently $\log R$ ) value are signal-like, while those with low $R$ value are QCD-like. Figures 6.13 and 6.14 show the $\log R$ distribution before and after $b$-tagging. A LHood $(\log R)$ cut of -2 typically gives a 40-50\% rejection of QCD for a signal loss of less than 5 percent.
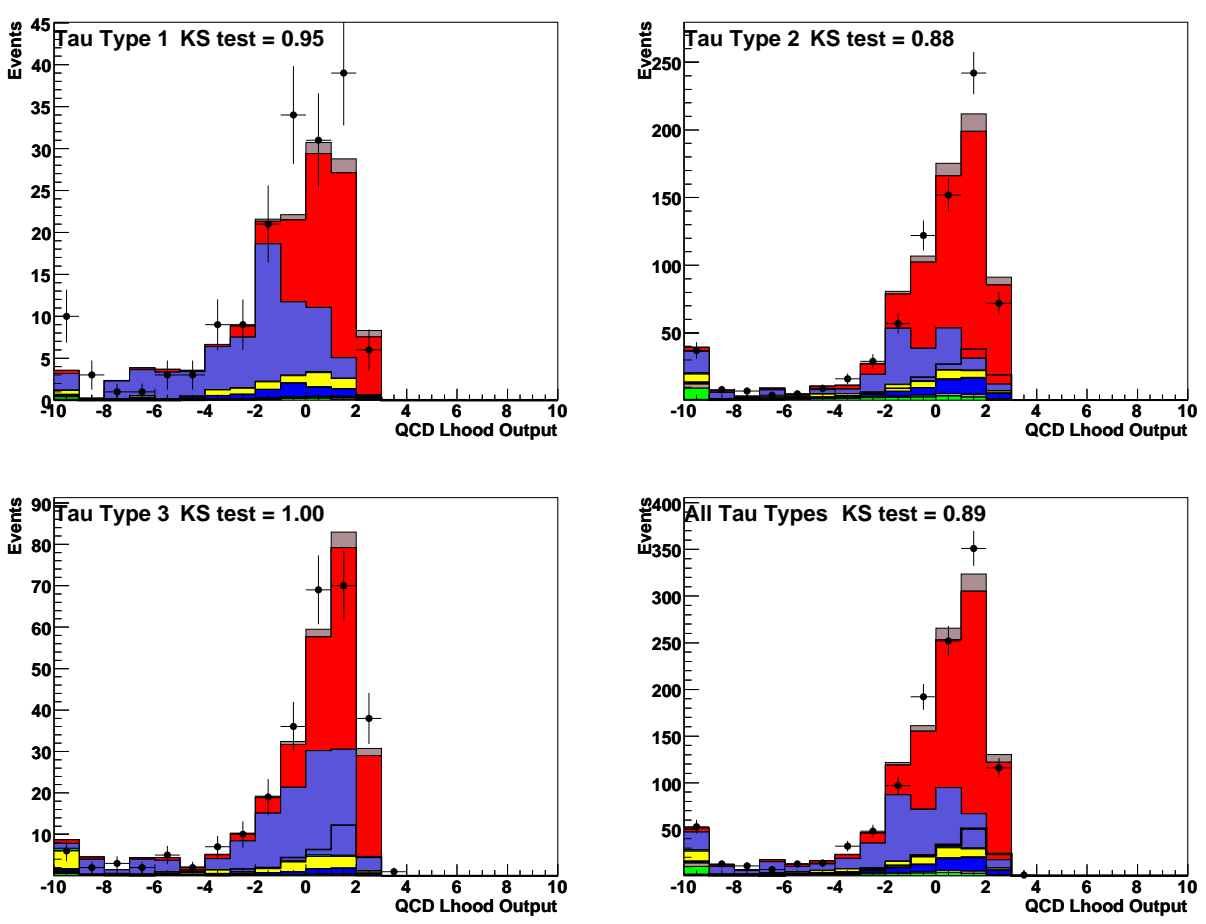

Figure 6.13: QCD likelihood $(\log R)$ output before $b$-tagging for: Type 1 taus (upper left), Type 2 (upper right), Type 3 (lower left), and all types (lower right).

We can then apply both the LHood and KNN to our sample and we should significantly reduce both backgrounds. Figure 6.15 shows the two dimensional plot with KNN on the vertical axis and LHood on the horizontal for the $b$ tagged samples. As we expect, since the signal should have both a high LHood and $\mathrm{KNN}$ value, it will gravitate to the upper right corner of the plot, while the QCD, having low LHood value, should be concentrated on the left side of the 

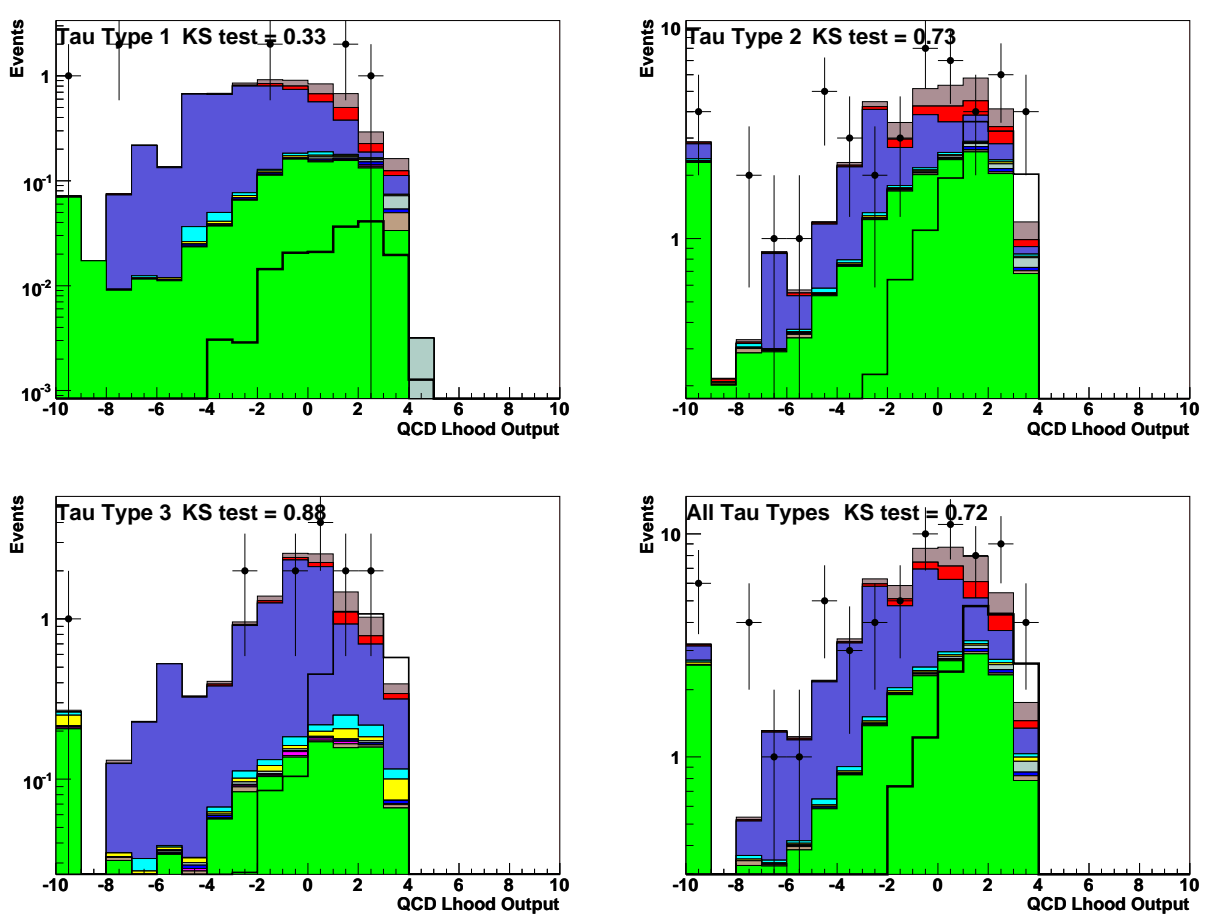

Figure 6.14: QCD likelihood $(\log R)$ output after $b$-tagging for: Type 1 taus (upper left), Type 2 (upper right), Type 3 (lower left), and all types (lower right). 
plot, and the $t \bar{t}$, having low KNN value, should be concentrated at the bottom of the plot. For each mass point we use a signal likelihood created from the signal distributions for that mass point only, and individually tailor the LHood and KNN cuts for each mass point. Table 6.9 shows the KNN and LHood cuts for each mass point. These values were chosen based on a limited optimization using the expected statistical significance as the optimization criterion.

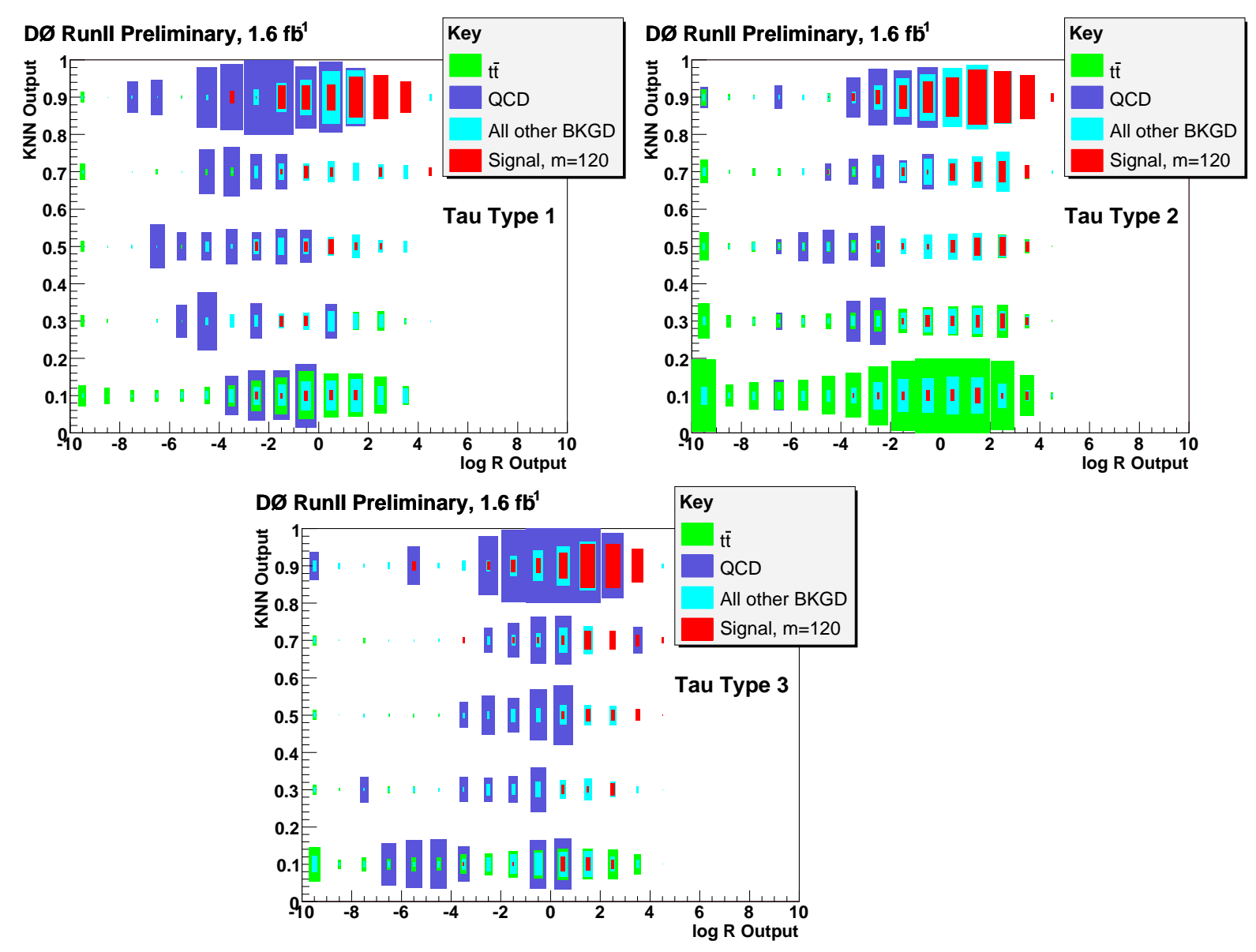

Figure 6.15: KNN output vs. QCD likelihood output with $120 \mathrm{GeV}$ signal for after $b$-tagging for: Type 1 taus (upper left), Type 2 (upper right), Type 3 (bottom.)

\subsubsection{Final Discriminant Variable}

After applying the cuts in Table 6.9 we transform the KNN-log $R$ plane into a one-dimensional variable according to the formula $(\log R+10) \times K N N$. It is 


\begin{tabular}{|c|c|c|c|c|c|c|}
\hline & \multicolumn{2}{|c|}{ Type 1 Cuts } & \multicolumn{2}{c|}{ Type 2 Cuts } & \multicolumn{2}{c|}{ Type 3 Cuts } \\
Higgs Mass & KNN & LHood & KNN & LHood & KNN & LHood \\
\hline 90 & 0.2 & -1 & 0.3 & -1 & 0.2 & -1 \\
100 & 0.3 & -2 & 0.3 & -1 & 0.2 & 0 \\
110 & 0.3 & -2 & 0.4 & -2 & 0.2 & -1 \\
120 & 0.2 & -2 & 0.35 & -2 & 0.2 & -1 \\
130 & 0.2 & -2 & 0.35 & -2 & 0.2 & -1 \\
140 & 0.3 & -2 & 0.35 & -2 & 0.2 & 0 \\
150 & 0.3 & -2 & 0.35 & -2 & 0.2 & -1 \\
160 & 0.2 & -2 & 0.3 & -3 & 0.2 & -1 \\
\hline
\end{tabular}

Table 6.9: Values of KNN and QCD likelihood cut for each mass point and tau type.

clear that it still retains good separation between the signal and two leading backgrounds. A signal-like event with both a high $\log R$ and KNN value will also have a high final value. Events with either a low KNN or $\log R$ value will also have a lower value in the final variable. Figure 6.16 shows the final variable before $b$-tagging; figure 6.18 shows the final variable after all cuts.

\subsection{Systematic Uncertainties}

The sources of systematic error in this analysis include the Jet Energy Scale(JES) uncertainty and Jet ID/resolution uncertainty, the uncertainty on the trigger efficiency, tau NN, luminosity, MC cross section, and QCD estimation. We calculate the uncertainties in the following ways:

- The JES uncertainty is determined by shifting the Jet Energy Scale by $\pm 1 \sigma$ in all Monte Carlo Samples and calculating new acceptances and limits. The same is done for the Jet Reconstruction efficiency uncertainty.

- We estimate the $b$-tagging efficiency uncertainty in a similar way by varying the Tag Rate Functions (TRFs) by $\pm 1 \sigma$ and calculating the new acceptances and limits.

- We apply the same method for the trigger uncertainty, varying the trigger weight on each $\mathrm{MC}$ event by $\pm 1 \sigma$ and calculating our new results. We also add an additional $3 \%$ flat systematic uncertainty to take into account impurities in the sample used to measure the efficiency.

- The Luminosity uncertainty is taken to be $6.1 \%$ [70]. 

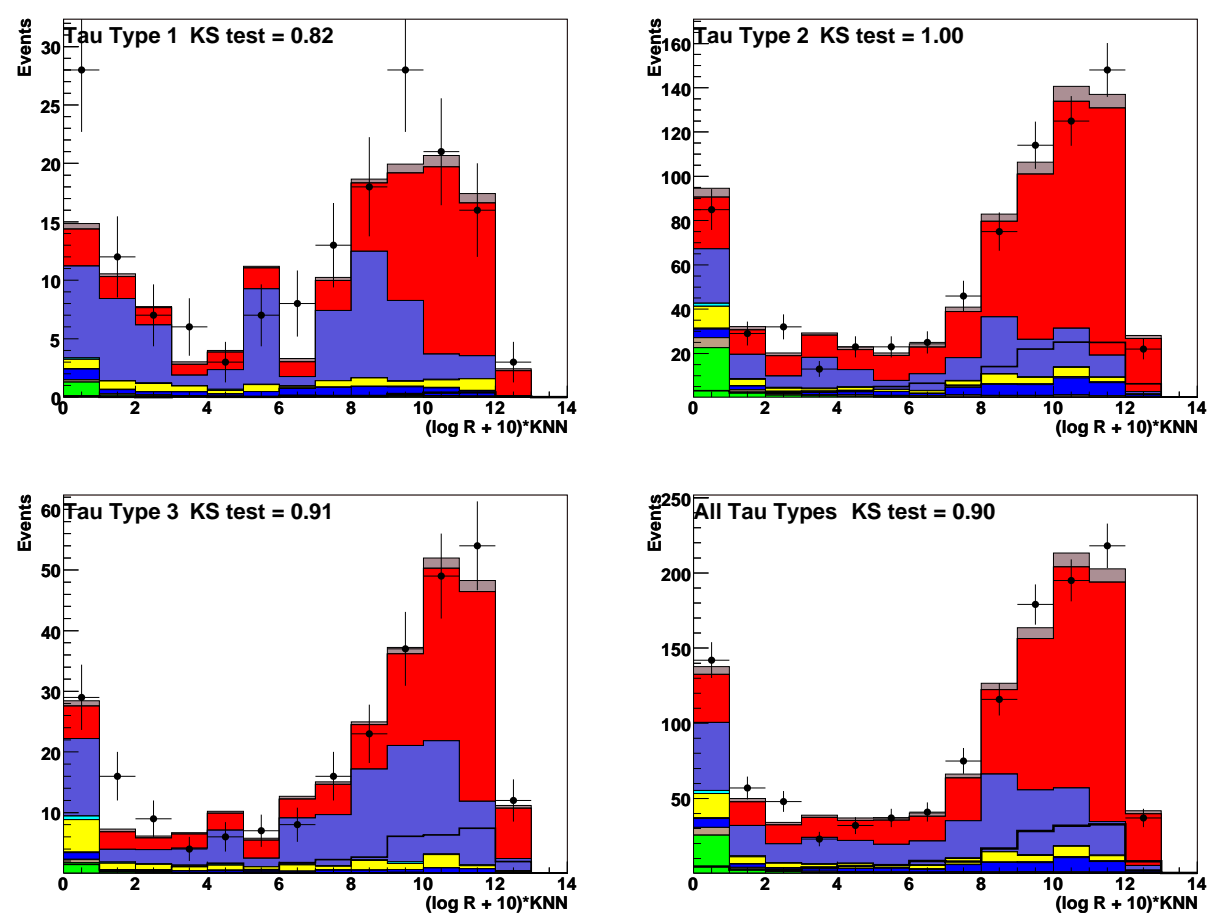

Figure 6.16: Final discriminant variable, with $120 \mathrm{GeV}$ signal overlay and using $120 \mathrm{GeV}$ QCD likelihood, before b-tagging, for: Type 1 taus (upper left), Type 2 (upper right), Type 3 (lower left), and all types (lower right).

- The $\tau$ ID/NN uncertainty is taken to be $8 \%$ in Type $1,4 \%$ in Type 2 , and $5 \%$ in Type 3 [71].

- The $\tau$ Energy Scale uncertainty is 3\% for all types [72].

- The $\mu$ ID uncertainty is comprised of systematic uncertainty on the muon ID $(0.4 \%)$, track match efficiency $(2.3 \%)$, and isolation data/MC efficiency measurement and correction $(3.8 \%)$. We combine these three in quadature to obtain a $4.5 \%$ systematic [44].

- We assign a 25\%,10\%, and 10\% systematic to the QCD calculation for tau types 1, 2, and 3, respectively, as described in Sec. 6.3.3.

- We assign a flat $10 \%$ uncertainty to the $W \rightarrow \ell \nu+$ lp MC cross section. We assign an $11 \%$ uncertainty on the $t \bar{t} \mathrm{MC}$ cross section and $6 \%$ to tbe diboson MC coming from the scale error and PDF error in the MCFM calculation [73]. We assign a $+2 \% /-5 \%$ systematic uncertainty to the 

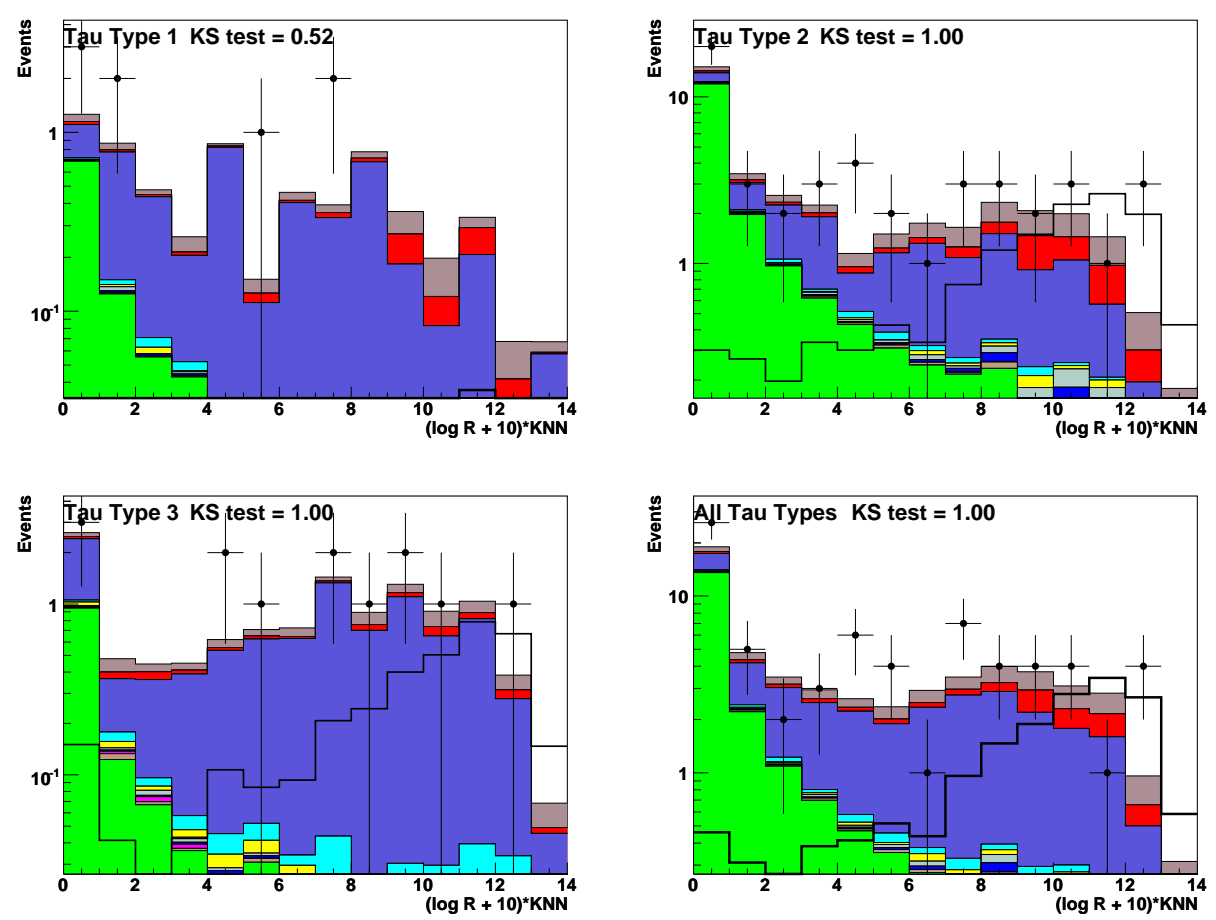

Figure 6.17: Final discriminant variable, with $120 \mathrm{GeV}$ signal overlay and using $120 \mathrm{GeV}$ QCD likelihood, after b-tagging, for: Type 1 taus (upper left), Type 2 (upper right), Type 3 (lower left), and all types (lower right).

$Z \rightarrow \mu \mu+\operatorname{lp}$ MC and $Z \rightarrow \tau \tau+$ lp MC cross sections, arising from the NNLO theory uncertainty [74],[75]. The $Z \rightarrow \tau \tau+\mathrm{HF}, Z \rightarrow \mu \mu+\mathrm{HF}$ and $W \rightarrow \ell \nu+\mathrm{HF}$ MC have a $30 \%$ uncertainty due to the uncertainty of the HF scale factor.

Since the JES, JSSR, Trigger, and b-tagging systematics are shape-driven, we denote them as "shape" in table 6.10 and do not quote a percentage. 

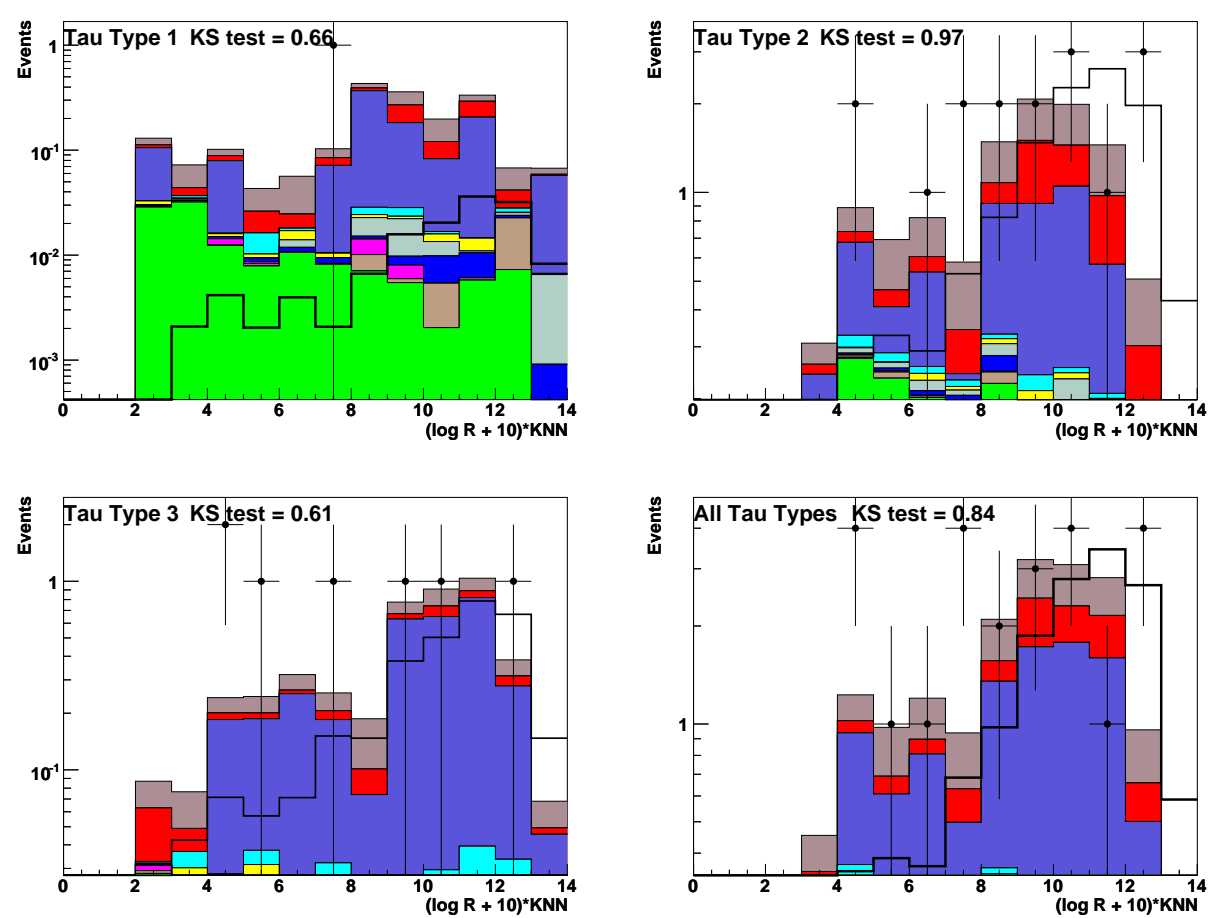

Figure 6.18: Final discriminant variable, with $120 \mathrm{GeV}$ signal overlay and using $120 \mathrm{GeV}$ QCD likelihood, after $b$-tagging and cuts in Table 6.9, for: Type 1 taus (upper left), Type 2 (upper right), Type 3 (lower left), and all types (lower right). 


\begin{tabular}{|c|c|c|c|c|c|c|c|c|c|c|}
\hline Sample & JES & Jet Res. & $b$-tagging & Trigger & Lumi. & $\tau$-ID & Tau ES & $\mu$-ID & $\overline{Q C D}$ & $\mathrm{MC} \sigma$ \\
\hline Signal & shape & shape & shape & shape $+3 \%$ & $6.1 \%$ & $3-8 \%$ & $3 \%$ & $4.5 \%$ & - & - \\
\hline$Z \rightarrow \tau \tau+\operatorname{lp}$ & shape & shape & shape & shape $+3 \%$ & $6.1 \%$ & $3-8 \%$ & $3 \%$ & $4.5 \%$ & - & $+2 /-5 \%$ \\
\hline$Z \rightarrow \tau \tau+\mathrm{HF}$ & shape & shape & shape & shape $+3 \%$ & $6.1 \%$ & $3-8 \%$ & $3 \%$ & $4.5 \%$ & - & $30 \%$ \\
\hline$Z \rightarrow \mu \mu+\operatorname{lp}$ & shape & shape & shape & shape $+3 \%$ & $6.1 \%$ & $3-8 \%$ & $3 \%$ & $4.5 \%$ & - & $+2 /-5 \%$ \\
\hline$Z \rightarrow \mu \mu+\mathrm{HF}$ & shape & shape & shape & shape $+3 \%$ & $6.1 \%$ & $3-8 \%$ & $3 \%$ & $4.5 \%$ & - & $30 \%$ \\
\hline$W+\mathrm{lp}$ & shape & shape & shape & shape $+3 \%$ & $6.1 \%$ & $3-8 \%$ & $3 \%$ & $4.5 \%$ & - & $10 \%$ \\
\hline$W+\mathrm{HF}+\mathrm{lp}$ & shape & shape & shape & shape $+3 \%$ & $6.1 \%$ & $3-8 \%$ & $3 \%$ & $4.5 \%$ & - & $30 \%$ \\
\hline & shape & shape & shape & shape $+3 \%$ & $6.1 \%$ & $3-8 \%$ & $3 \%$ & $4.5 \%$ & - & $11 \%$ \\
\hline$W W / W Z$ & shape & shape & shape & shape $+3 \%$ & $6.1 \%$ & $3-8 \%$ & $3 \%$ & $4.5 \%$ & - & $6 \%$ \\
\hline Single $t$ & shape & shape & shape & shape $+3 \%$ & $6.1 \%$ & $3-8 \%$ & $3 \%$ & $4.5 \%$ & - & $12 \%$ \\
\hline QCD Type 1 & - & - & - & - & - & - & - & - & $25 \%$ & - \\
\hline QCD Type 2 & - & - & - & - & - & - & - & - & $10 \%$ & - \\
\hline QCD Type 3 & - & - & - & - & - & - & - & - & $10 \%$ & - \\
\hline
\end{tabular}

Table 6.10: Systematic uncertainties applied to each signal and background sample. For shape dependent systematics, denoted as "shape" in the table, we vary the effect by $\pm 1 \sigma$, then use the resulting distributions as inputs to the limit setting program (section 7.1). Those systematics expressed as a percentage are taken as flat systematics (no shape dependence) and are input into the limit setting program accordingly. The dashes ("-") mean that the systematic in that column is not applied to the MC sample in that row. 


\begin{tabular}{|l|c|c|c|c|}
\hline \multicolumn{2}{|l|}{ Tau Type 1 } & Tau Type 2 & Tau Type 3 & All Tau Types \\
\hline$\mu, \tau$ preselection $+\geq 1$ jet \\
\hline Data & 170 & 760 & 270 & 1200 \\
Total pred. & $144.0 \pm 8.7$ & $779.8 \pm 16.1$ & $265.9 \pm 12.3$ & $1189.7 \pm 22.0$ \\
$Z \rightarrow \tau \tau$ & $62.6 \pm 2.4$ & $462.7 \pm 7.0$ & $120.2 \pm 3.5$ & $645.4 \pm 8.2$ \\
$Z \rightarrow \tau \tau+\mathrm{hf}$ & $4.9 \pm 0.3$ & $36.5 \pm 0.7$ & $8.8 \pm 0.3$ & $50.1 \pm 0.8$ \\
$Z \rightarrow \mu \mu$ & $6.8 \pm 0.7$ & $40.0 \pm 1.9$ & $5.6 \pm 0.7$ & $52.4 \pm 2.1$ \\
$Z \rightarrow \mu \mu+\mathrm{hf}$ & $0.3 \pm 0.1$ & $3.0 \pm 0.2$ & $0.4 \pm 0.1$ & $3.7 \pm 0.3$ \\
$Z \rightarrow e e$ & $0 \pm 0$ & $3.6 \pm 2.0$ & $0 \pm 0$ & $3.6 \pm 2.0$ \\
$W+\mathrm{LP}$ & $7.0 \pm 1.0$ & $36.0 \pm 2.5$ & $16.7 \pm 1.6$ & $59.7 \pm 3.2$ \\
$W+\mathrm{HF}$ & $0.6 \pm 0.1$ & $3.8 \pm 0.3$ & $2.0 \pm 0.2$ & $6.4 \pm 0.4$ \\
$t \bar{t}$ & $1.7 \pm 0.1$ & $29.6 \pm 0.4$ & $2.2 \pm 0.1$ & $33.5 \pm 0.5$ \\
Single $t$ & $0.03 \pm 0.01$ & $0.125 \pm 0.02$ & $0.08 \pm 0.01$ & $0.23 \pm 0.03$ \\
$W W / W Z$ & $0.9 \pm 0.1$ & $12.9 \pm 0.5$ & $1.5 \pm 0.2$ & $15.3 \pm 0.4$ \\
$\mathrm{QCD}$ & $59.3 \pm 8.2$ & $155.1 \pm 14.1$ & $108.4 \pm 11.6$ & $322.8 \pm 20.0$ \\
Signal, M=120 & $1.6 \pm 0.1$ & $12.4 \pm 0.4$ & $3.4 \pm 0.2$ & $17.4 \pm 0.5$ \\
\hline$b$-tagging & \multicolumn{4}{|c|}{} \\
\hline Data & 8 & 50 & 13 & 71 \\
Total pred. & $7.45 \pm 0.80$ & $38.39 \pm 1.30$ & $12.36 \pm 1.10$ & $58.20 \pm 1.88$ \\
$Z \rightarrow \tau \tau$ & $0.41 \pm 0.07$ & $2.90 \pm 0.21$ & $0.59 \pm 0.03$ & $3.90 \pm 0.22$ \\
$Z \rightarrow \tau \tau+\mathrm{hf}$ & $0.69 \pm 0.05$ & $5.14 \pm 0.12$ & $1.25 \pm 0.06$ & $7.09 \pm 0.14$ \\
$Z \rightarrow \mu \mu$ & $0.035 \pm 0.004$ & $0.25 \pm 0.04$ & $0.026 \pm 0.003$ & $0.31 \pm 0.04$ \\
$Z \rightarrow \mu \mu+\mathrm{hf}$ & $0.027 \pm 0.007$ & $0.27 \pm 0.02$ & $0.024 \pm 0.006$ & $0.32 \pm 0.03$ \\
$Z \rightarrow e e$ & $0 \pm 0$ & $0 \pm 0$ & $0 \pm 0$ & $0 \pm 0$ \\
$W+\mathrm{LP}$ & $0.035 \pm 0.005$ & $0.24 \pm 0.03$ & $0.15 \pm 0.03$ & $0.42 \pm 0.04$ \\
$W+\mathrm{HF}$ & $0.06 \pm 0.01$ & $0.44 \pm 0.04$ & $0.18 \pm 0.02$ & $0.68 \pm 0.05$ \\
$t \bar{t}$ & $0.99 \pm 0.05$ & $17.30 \pm 0.23$ & $1.26 \pm 0.05$ & $19.55 \pm 0.24$ \\
Single $t$ & $0.010 \pm 0.003$ & $0.052 \pm 0.007$ & $0.03 \pm 0.01$ & $0.09 \pm 0.01$ \\
$W W / W Z$ & $0.031 \pm 0.015$ & $0.26 \pm 0.03$ & $0.032 \pm 0.005$ & $0.33 \pm 0.04$ \\
$\mathrm{QCD}$ & $4.23 \pm 0.49$ & $10.98 \pm 0.86$ & $8.53 \pm 0.72$ & $23.74 \pm 1.33$ \\
Signal, M=120 & $0.52 \pm 0.05$ & $4.19 \pm 0.15$ & $1.14 \pm 0.08$ & $5.85 \pm 0.17$ \\
\hline
\end{tabular}

Table 6.11: The predicted and observed event yields, with statistical errors, as a function of the selection requirements. We discuss the systematic errors in section 6.5. In this table we assume a signal cross section of $1 \mathrm{pb}$. 


\begin{tabular}{|l|c|c|c|}
\hline & \multicolumn{3}{|c|}{ Accepted Events/Total (in \%) } \\
\hline Higgs mass (GeV) & Tau Type 1 & Tau Type 2 & Tau Type 3 \\
\hline$\mu, \tau$ preselection+ +1 jet \\
\hline 90 & $0.076 \%$ & $0.428 \%$ & $0.122 \%$ \\
100 & $0.083 \%$ & $0.530 \%$ & $0.144 \%$ \\
110 & $0.102 \%$ & $0.629 \%$ & $0.175 \%$ \\
120 & $0.102 \%$ & $0.768 \%$ & $0.213 \%$ \\
130 & $0.102 \%$ & $0.748 \%$ & $0.210 \%$ \\
140 & $0.116 \%$ & $0.875 \%$ & $0.215 \%$ \\
150 & $0.127 \%$ & $0.944 \%$ & $0.261 \%$ \\
160 & $0.130 \%$ & $0.937 \%$ & $0.285 \%$ \\
\hline$b$-tagging & \multicolumn{3}{|c|}{} \\
\hline 90 & $0.025 \%$ & $0.138 \%$ & $0.036 \%$ \\
100 & $0.024 \%$ & $0.176 \%$ & $0.046 \%$ \\
110 & $0.033 \%$ & $0.205 \%$ & $0.058 \%$ \\
120 & $0.032 \%$ & $0.260 \%$ & $0.071 \%$ \\
130 & $0.034 \%$ & $0.245 \%$ & $0.071 \%$ \\
140 & $0.037 \%$ & $0.292 \%$ & $0.072 \%$ \\
150 & $0.043 \%$ & $0.316 \%$ & $0.089 \%$ \\
160 & $0.041 \%$ & $0.312 \%$ & $0.096 \%$ \\
\hline
\end{tabular}

Table 6.12: Percentage of total events per mass point accepted by each tau type. The signal MC events are generated with inclusive tau decays, not only the $\mu \tau_{\text {had }}$ final state. 


\section{Chapter 7}

\section{Results}

The previous chapter developed the procedure for the analysis and introduced the final discriminant variable. In this chapter we set limits on the $\phi b$ cross section times branching ratio of $\phi \rightarrow \tau^{+} \tau^{-}$and translate the cross section limit into limits on MSSM parameters.

\subsection{Cross Section Limit}

In the absence of statistically significant signal we set limits on $\phi b$ production. We use a modified Frequentist approach [76] to set the cross section times branching ratio limits. To maximize sensitivity and treat per-channel fluctuations individually, we treat each tau type as a separate channel. The key quantity in the limit calculation is the log likelihood ratio of the signal+background and background-only hypotheses, defined as

$$
\operatorname{LLR}(\boldsymbol{s}, \boldsymbol{b}, \boldsymbol{d})=\sum_{i=1}^{3} \sum_{j=0}^{N_{b i n s}} s_{i j}-d_{i j} \ln \left(1+\frac{s_{i j}}{b_{i j}}\right)
$$

where the first sum is over the three tau types, $N_{\text {bins }}$ is the number of bins in the distribution used for the final discriminant variable (sec. 6.4.3), and $s_{i j}, b_{i j}$, and $d_{i j}$ are the numbers of signal, MC background, and data events in the $j$-th bin of the $i$-th tau type.

The DØ limit-setting program [77] builds up LLR distributions by running so-called "pseudoexperiments": running a random Poisson trial on each background and signal distribution in each bin of the final variable, with the Poisson mean of each background or signal distribution smeared according to the systematic uncertainties on that background. The set of background and/or signal distributions resulting from one trial of each is one pseudoex- 
periment. The collection can also be called pseudodata.

We perform a "best fit" of the pseudodata to the actual data by minimizing the following $\chi^{2}$ function, called the Profile Likelihood:

$$
\chi^{2}=2 \sum_{i=0}^{N b i n s}\left(B_{i}-D_{i}\right)-D_{i} \ln \left(\frac{B_{i}}{D_{i}}\right)+\sum_{k=0}^{N s y s t} S_{k}^{2}
$$

where $B_{i}$ is the pseudodata in the $i$-th bin (each background is varied separately according to its uncertainties), $D_{i}$ is the data in the $i$-th bin, and the $S_{k}$ terms are scale factors applied to each of the $k$ sources of systematic uncertainty. There is a penalty term in the fit (the $\sum_{k=0}^{N s y s t} S_{k}^{2}$ term) if the backgrounds start to vary by more than the assigned uncertainties.

Then we redefine the LLR as

$$
L L R_{\text {new }}=\chi_{\min }^{2}(H 1)-\chi_{\min }^{2}(H 0)
$$

where $\mathrm{H} 1$ is the signal+background hypothesis, and $\mathrm{H} 0$ is the background-only, or null, hypothesis. We do the $\chi^{2}$ minimization of equation 7.2 by varying the $S_{k}$ terms for both hypotheses for each pseudoexperiment, calculate the LLR as above, and place the LLR into a histogram. We perform two such fits per pseudoexperiment, one where the pseudodata includes a signal and background contribution, and one where the pseudodata is from the background only. We run 50,000 pseudoexperiments for each mass point. Figure 7.1 shows the LLR distributions from equation 7.3 along with the observed LLR (calculated with eq. 7.1 ) for each mass point.

We can then define two statistics, $C L_{s b}$ and $C L_{B}$, which are the fraction of pseudoexperiments with LLR values greater than the observed LLR value in the signal+background and background-only hypotheses, respectively. From these we can define $C L_{s}$ as $\frac{C L_{s b}}{C L_{B}}$. The $95 \%$ confidence level limit (i.e. the quoted cross section limit) is then defined as the value of the signal cross section where $1-C L_{s}=0.05$. Figure 7.2 shows the $1-C L_{s}$ value at each mass point for the nominal input cross section. The limit setting program slowly increases the signal cross section, calculating the new LLR values, $C L_{s b}, C L_{B}$, and $1-C L_{s}$ for each iteration, until $1-C L_{s}=0.05$. That value of the cross section is then the limit. Figure 7.3 shows the expected and observed cross section times branching ratio limits for each mass point from 90 to $160 \mathrm{GeV}$. 

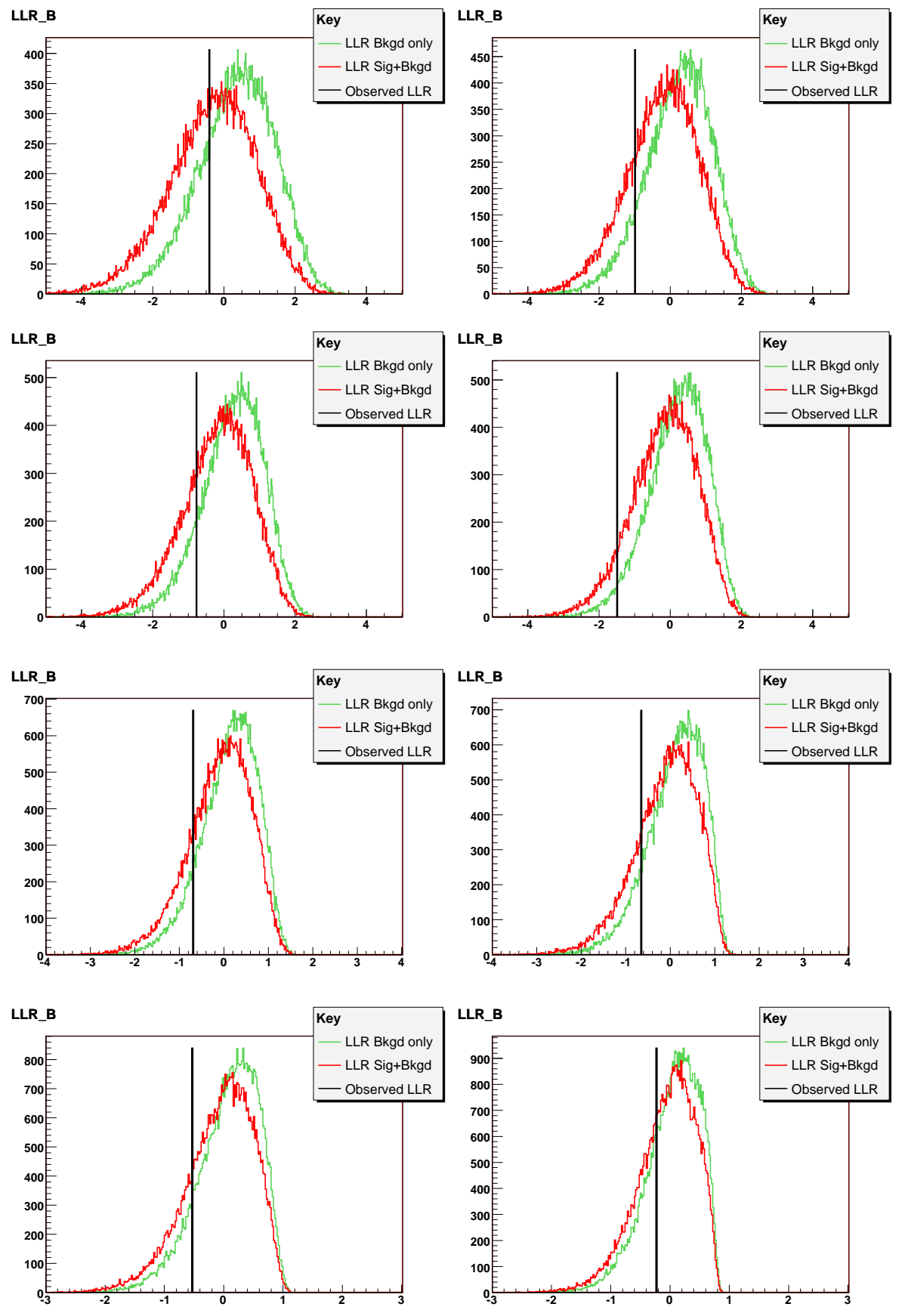

Figure 7.1: LLR distributions used in the limit setting for each mass point. Row 1: $90 \mathrm{GeV}$ (left), $100 \mathrm{GeV}$ (right). Row 2: $110 \mathrm{GeV}$ (left), $120 \mathrm{GeV}$ (right). Row 3: $130 \mathrm{GeV}$ (left), $140 \mathrm{GeV}$ (right). Row 4: $150 \mathrm{GeV}$ (left), 160 $\mathrm{GeV}$ (right). 


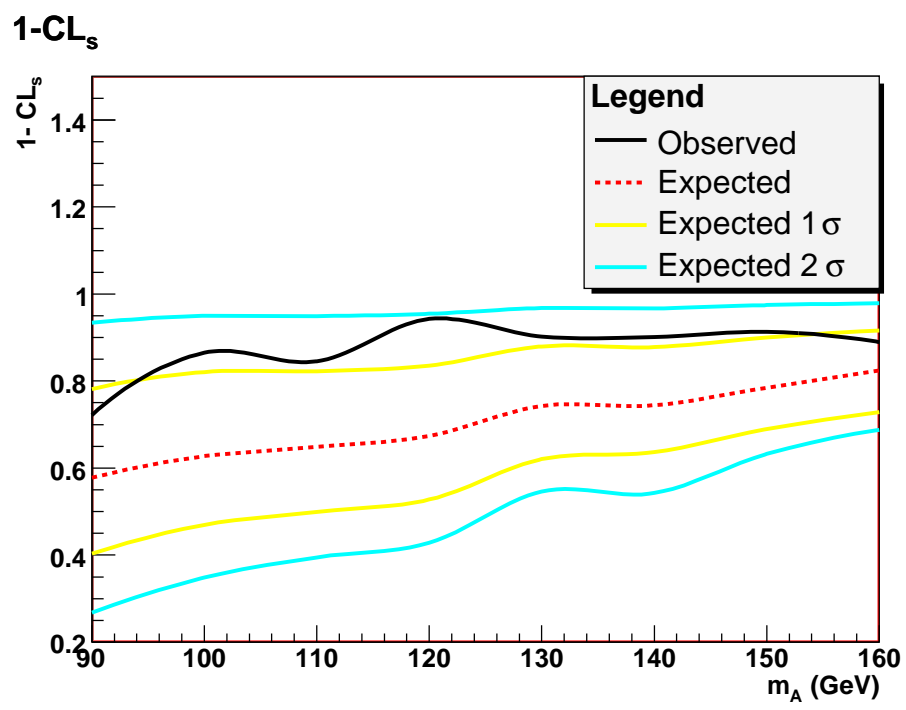

Figure 7.2: $1-C L_{s}$ vs. Higgs mass for the nominal input cross section. The limit setting program slowly increases the signal cross section until this value is 0.05 at that mass point.

\subsection{Interpretation within the MSSM}

The cross section times branching ratio limit is independent of any particular SUSY model. To translate the cross section limits into limits on SUSY parameters such as $\tan \beta$, one needs to find a set of parameters that gives a cross section times branching ratio equal to the limit. We set limits on $\tan \beta$ as a function of the pseudoscalar Higgs mass $m_{A}$. We fix two SUSY parameters, the amount of Higgs mixing and the Higgsino mass parameter $\mu$; then we use Feynhiggs to find the $\tan \beta$ value that gives the same $\phi b \sigma \times B R$ as our limit. That is then the $\tan \beta$ limit. The final $\tan \beta$ vs. $m_{A}$ limits are shown in figure 7.4. We set limits in four different MSSM scenarios: no Higgs mixing, $\mu=+200 \mathrm{GeV}$; no Higgs mixing, $\mu=-200 \mathrm{GeV}$; maximal Higgs mixing, $\mu=+200 \mathrm{GeV}$, and maximal Higgs mixing, $\mu=-200 \mathrm{GeV}$. 


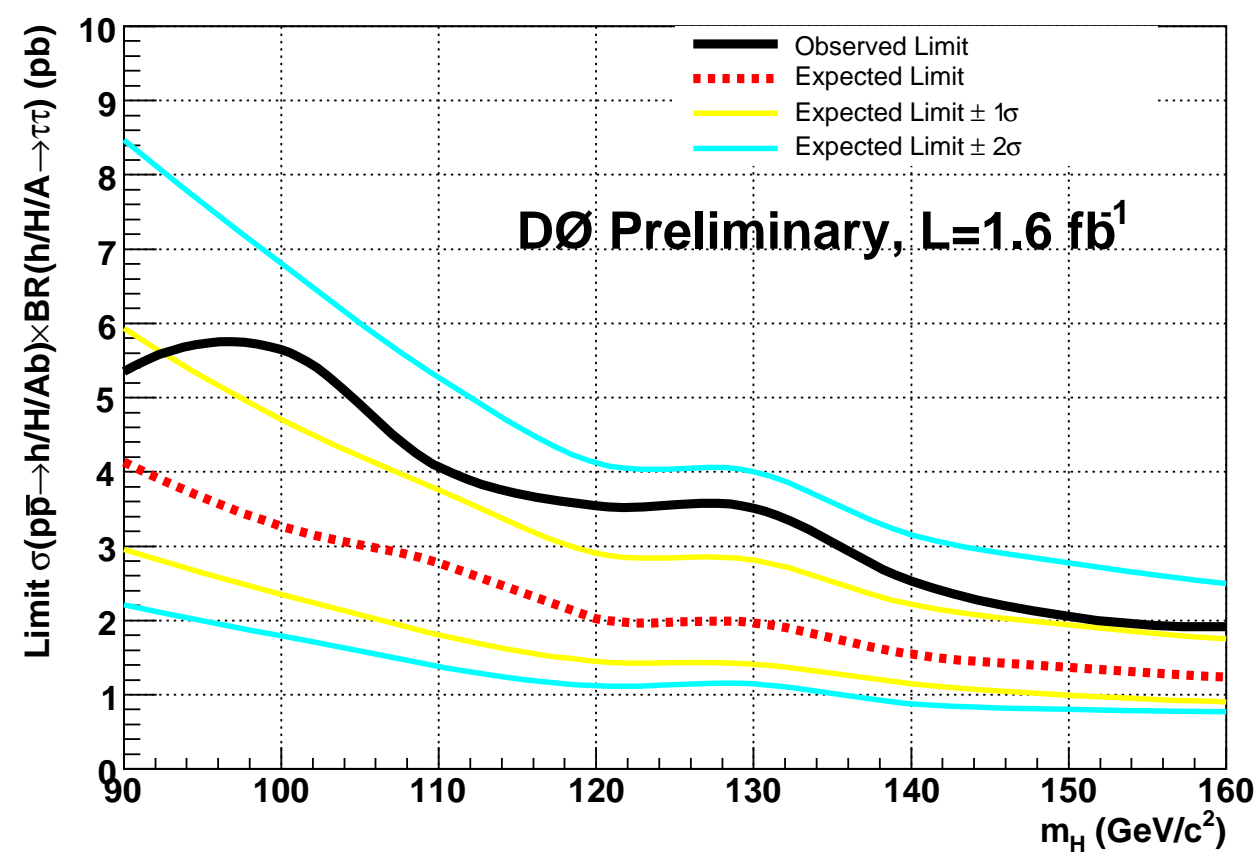

Figure 7.3: Expected and observed cross section $\times$ BR limits vs. Higgs mass. 

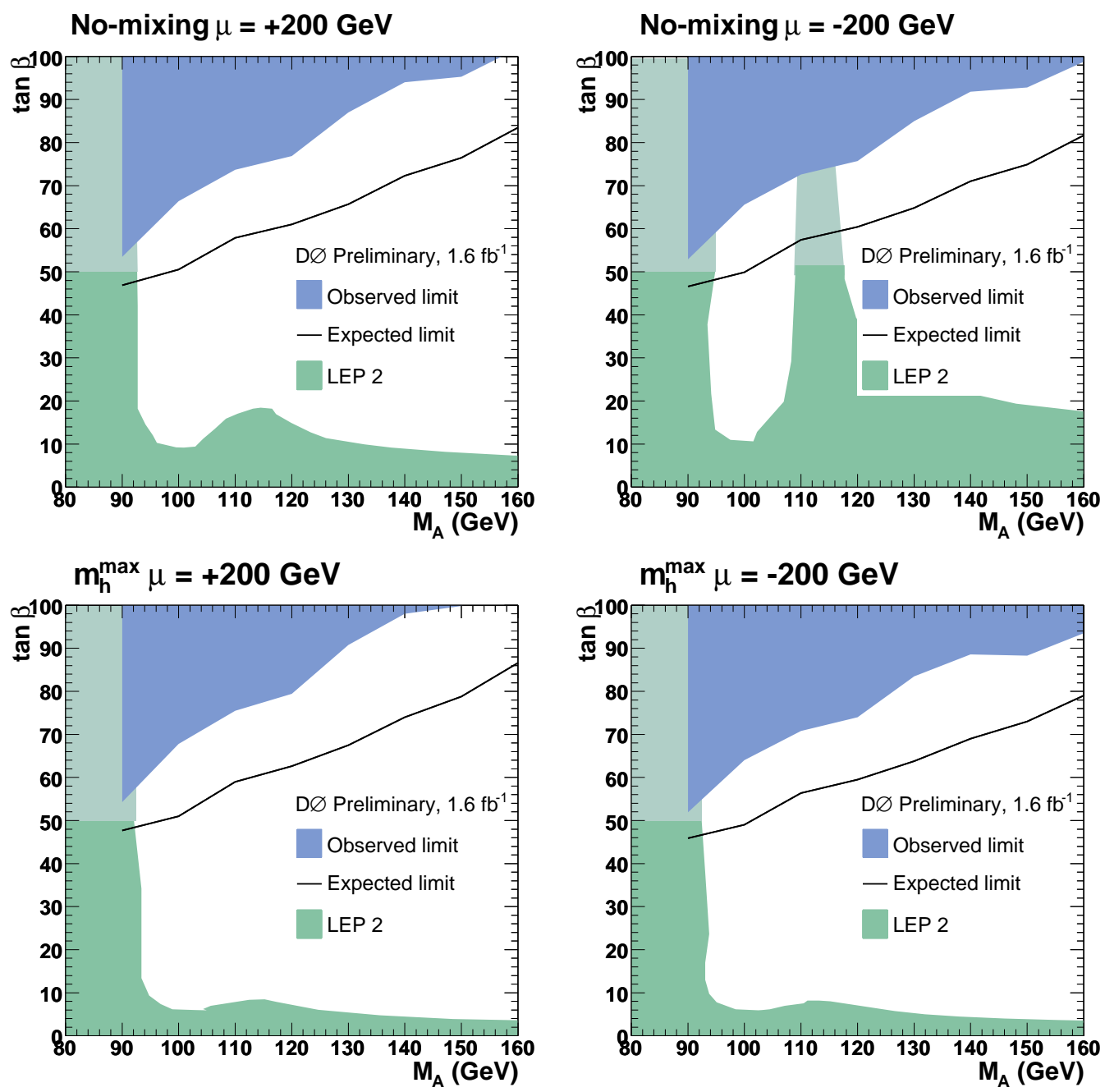

Figure 7.4: Limits on $\tan \beta$ vs. $M_{A}$ for the no-mixing, $\mu>0$ case (upper left); no-mixing, $\mu<0$ case (upper right); maximal-mixing, $\mu>0$ case (lower left); maximal-mixing, $\mu<0$ case (lower right). 


\section{Chapter 8}

\section{Conclusions}

In this final chapter we present a brief overview of the results of our analysis, and conclude with a look at what the future holds for this channel, and SUSY Higgs searches at DØ.

\subsection{Conclusions}

We have performed a search for neutral Higgs bosons decaying to tau pairs where the Higgs is produced in association with a $b$-quark. We use $1.6 \mathrm{fb}^{-1}$ of data taken with the $\mathrm{D} \varnothing$ detector at the Fermilab Tevatron. We observe no statistically significant excess in data over the predicted background. In the absence of a signal we set limits on $\sigma(p \bar{p} \rightarrow \phi b) \times \mathrm{BR}(\phi \rightarrow \tau \tau)$ (Figure 7.3) and translate those limits to limits in the $\tan \beta$ vs. $m_{A}$ plane in MSSM parameter space.

We are able to exclude a significant portion of the space. In all four scenarios considered we can exclude $\tan \beta>103$ at all mass points. Conversely, we can exclude Higgs masses $<160 \mathrm{GeV}$ in MSSM models having a $\tan \beta>103$.

\subsection{Outlook}

While this analysis has excluded a significant portion of $\tan \beta$ vs. $m_{A}$ parameter space, there is potential for further improvement. The next iteration of this analysis at $\mathrm{D} \varnothing$ could take advantage of significant improvements in the dataset, triggers, and analysis techniques. It could also combine different final states such as $\tau_{e} \tau_{\text {had }} b$ or $\tau_{\text {had }} \tau_{\text {had }} b$.

While we have only analyzed the first $1.6 \mathrm{fb}^{-1}$ of RunIIb data at $\mathrm{D} \varnothing$, there is an additional $2 \mathrm{fb}^{-1}$ available for analysis as of October $2008 ; 1 \mathrm{fb}^{-1}$ of RunIIa data, and $1 \mathrm{fb}^{-1}$ of RunIIb data taken from April-September 2008. As 
of November 2008, a separate analysis in this channel using the RunIIa data is nearly complete, and it will be combined with this analysis for publication with a $2.7 \mathrm{fb}^{-1}$ total dataset. Future limits will improve significantly simply by repeating this analysis on the larger dataset, including the additional RunIIb data. Naively one would expect, in the absence of signal, the limits to improve by the square root of the ratio of integrated luminosity of the larger dataset to the smaller, i.e. $\sqrt{\mathcal{L}_{\text {int Larger }} / \mathcal{L}_{\text {int Smaller }}}$.

Combining different final states is another way to extend the reach of the $\phi b$ search. As of October 2008 work has begun on a $\phi b$ search in a final state where the Higgs decays to a tau pair, and one of the taus decays hadronically while the other decays to an electron ( the $\tau_{e} \tau_{\text {had }} b$ final state.) This is clearly complimentary to our channel and should result in a significant improvement after combining the two analyses. The challenges to that analysis include the multijet background estimate as well as the $Z \rightarrow$ ee background, where one of the electrons fakes a tau. Another interesting final state is where both taus decay hadronically, $\tau_{\text {had }} \tau_{h a d} b$. This offers the best branching ratio as we saw in section 1.4. Such an analysis has never been tried at D $\varnothing$ despite the obvious benefit, as it has not been clear how to estimate the multijet background as well as due to fear that the background, however well-modeled it might be, would overwhelm the signal.

During 2007 and early 2008, however, D $\varnothing$ made extensive changes to its trigger list, including adding dedicated triggers for this final state. There are now triggers requiring two hadronic taus and triggers with two hadronic taus plus an additional jet; these have somewhat looser cuts on the taus than the triggers without the jet. They are better able to reject background than v15 hadronic tau triggers, yet retain a high efficiency. The key is in improved trackjet and track-tau matching as well as tightening the tau ratio requirements (see section 3.2.2) to the point just before the efficiency on real taus begins to drop, but the background rejection is large. Using these triggers may reduce the multijet background enough to where the $\tau_{\text {had }} \tau_{\text {had }} b$ final state provides significant additional sensitivity. Additionally, the muon +tau and electron + tau triggers also have tightened the tau requirements in a similar way, preserving their efficiency but increasing their background rejection.

By taking the improvements in the trigger list and analysis techniques (including multivariate methods) and applying them to the large and growing DØ dataset, future analyzers should be able to significantly improve upon the limits presented here. By combining the results from multiple $\phi b$ final states, including $b b \bar{b}$, we can hope to see groundbreaking results that deepen our understanding of the fundamental nature of our world. 


\section{Bibliography}

[1] C. Amsler et al., Phys. Lett. B 667 (2008).

[2] C. Quigg, Gauge Theories of the Strong, Weak, and Electromagnetic Interactions (Westview Press, 1983).

[3] P. Higgs, Phys. Rev. Lett. 13, 508 (1964).

[4] D. Griffiths, Introduction to Elementary Particles (John Wiley and Sons, Inc., 1987).

[5] The LEP Higgs Working Group, Phys. Lett. B 565, 61 (2003).

[6] The TEVNPH Working Group, FERMILAB-PUB-08-270-E (2008).

[7] J. Terning, Modern Supersymmetry: Dynamics and Duality (Oxford Science Publications, 2006).

[8] M. E. Peskin, SLAC-PUB-13079 (2008).

[9] I. J. R. Aitchison, hep-ph/0505105 (2005).

[10] G. Kane, Supersymmetry: squarks, photinos, and the unveiling of the ultimate laws of nature (Perseus Publishing, 2000).

[11] A. Haas, Ph.D. thesis, University of Washington, Seattle, WA (2004).

[12] M. Carena, J. Conway, H. Haber, J. Hobbs, et al., Report of the tevatron higgs working group, FERMILAB-Conf-00/279-T (2000).

[13] J. Campbell, R. Ellis, F. Maltoni, and S. Willenbrock, Phys. Rev. D 67, 095002 (2003).

[14] V. M. Abazov et al., Phys. Rev. Lett. 95, 151801 (2005).

[15] V. M. Abazov et al., FERMILAB-PUB-08/142-E (accepted by Phys. Rev. Lett.) (2008). 
[16] V. M. Abazov et al., DØInternal Note 5726-CONF (2008).

[17] The CDF Collaboration, CDF Note 9284 (2008).

[18] V. Abazov et al., Search for neutral higgs bosons at high $\tan \boldsymbol{\beta}$ in the $\boldsymbol{b}(\boldsymbol{h} / \boldsymbol{h} / \boldsymbol{a}) \rightarrow \boldsymbol{\tau} \boldsymbol{\tau}$ channel, FERMILAB-PUB-08/451-E (2008).

[19] Fermi National Accelerator Laboratory, URL http://www.fnal.gov/ $\mathrm{pub/about/whatis/history.html.}$

[20] M. Cooke, Ph.D. thesis, Rice University, Houston, TX (2007).

[21] S. Abachi et al., Nucl. Instrum. Methods Phys. Res. A 338, 185 (1994).

[22] V. M. Abazov et al., Nucl. Inst. Meth. A 565, 463 (2006).

[23] V. Abazov et al., The muon system of the run ii DØ detector, physics/0503151 (2005).

[24] J. Anderson et al., IEEE Trans. on Nucl. Sci. 51, 345 (2004).

[25] M. Abolins et al., Nucl. Instr. and Methods Phys. Res. A 289, 542 (1990).

[26] M. Abolins et al., Nucl. Instrum. and Methods A 584, 75 (2007).

[27] J. Bystricky et al., IEEE Trans. on Nucl. Sci. 51, 351 (2004).

[28] The D0 collaboration, Run iib upgrade technical design report, FermilabPub-02/327-E (2002).

[29] S. E. Burke, Ph.D. thesis, University of Arizona, Tuscon, AZ (2007).

[30] T. Adams et al., submitted to Nucl. Inst. and Methods A (2008).

[31] R. Angstadt et al., IEEE Trans. on Nucl. Sci. 51, 454 (2004).

[32] P. Calfayan, DØ Internal Note 5329 (2007).

[33] A. Khanov, Htf: histogramming method for finding tracks. the algorithm description., DØ Internal note 3778 (2000).

[34] D. Adams, Finding tracks., DØ Internal Note 2918 (1998).

[35] H. Greenlee, The DØ kalman track fit., DØ Internal Note 4303 (2004).

[36] A. Schwartzmann and C. Tully, Primary vertex reconstruction by means of adaptive vertex fitting, D $\varnothing$ Internal Note 4918 (2005). 
[37] Y. Peters, A. Schwartzman, and M. Strauss, Certification of the adaptive primary vertex in p17, DØ Internal Note 5192 (2006).

[38] A. Schwartzman and M. Narain, Probabilistic primary vertex selection, DØ Internal Note 4042 (2002).

[39] J.-R. Vlimant, U. Bassler, G. Bernardi, and S.Trincz-Duvoid, Technical description of the 422 algorithm for the calorimeter noise suppression, $\mathrm{D} \varnothing$ Internal Note 4146 (2003).

[40] E. Busato and B. Andrieu, Jet algorithms in d0 runii software: Description and user's guide, DØ Internal Note 4457 (2004).

[41] The DØ Collaboration, URL http://www-d0.fnal.gov/phys_id/jes/ public_RunIIa/.

[42] D. Chakraborty et al., Reconstruction of $\tau$ leptons in hadronic final states at DØ run 2, DØ Internal note 4210 (2003).

[43] S. Protopopescu and P. Svoisky, $\tau$ identification with neural networks for p17 data, DØ Internal Note 5094 (2006).

[44] P. Calfayan et al., DØ Internal Note 5157 (2007).

[45] T.Scanlon, A neural network b-tagging tool, DØ Internal Note 4889 (2005).

[46] T. Gadfort et al., Performance of the DØ nn b-tagging tool on p20 data, DØ Internal Note 5554 (2008).

[47] T. Sjostrand, L. Lonnblad, S. Mrenna, and P. Skands, Pythia 6.3 physics and manual, hep-ph/0308153.

[48] M. Mangano, M. Moretti, F. Piccinini, R. Pittau, and A. Polosa, JHEP 0307 (2003).

[49] H. Lai et al., Phys. Rev. D 55, 1280 (1997).

[50] E. Boos et al. (CompHEP Collaboration), Nucl. Instrum. Meth. A 534, 250 (2004).

[51] A. Pukhov et al., INP MSU report 98-41/542, URL http://comphep. sinp.msu.ru.

[52] A. Belyaev et al., Comphep - pythia interface: integrated package for the collision events generation based on exact matrix elements, in: Advanced computing and analysis techniques in physics research (2000). 
[53] S. Jadach, Z. Was, R. Decker, and J. H. Kuhn, Comput. Phys. Commun. 76, 361 (1993).

[54] D. J. Lange, Nucl. Instrum. Meth. A 462, 152 (2001).

[55] R. Brun and F. Carminati, CERN Program Library Long Writeup W5013 (unpublished) (1993).

[56] M. Owen, A. Patwa, S. Söldner-Rembold, and W. C. Yang, DØ Internal Note 5708 (2008).

[57] A. Harel and J. Kvita, p20 jetid efficiencies and scale factors, DØ Internal Note 5634 (2008).

[58] H. Schellman, DØ Internal Note 5540 (2007).

[59] H. Schellman, DØ Internal Note 5142 (2006).

[60] V. M. Abazov et al., Phys. Rev. D 76, 012003 (2007).

[61] M. Shamim and T. Bolton, DØ Internal Note 5565 (2008).

[62] D. Boline, DØ Internal Note 5569 (2008).

[63] The DØ V+jets Group, Private communication.

[64] M. Frank, T. Hahn, S. Heinemeyer, W. Hollik, H. Rzehak, and G. Weiglein, JHEP 0702 (2007).

[65] G. Degrassi, S. Heinemeyer, W. Hollik, P. Slavich, and G. Weiglein, Eur. Phys. J. C28, 133 (2003).

[66] S. Heinemeyer, W. Hollik, and G. Weiglein, Eur. Phys. J. C9, 343 (1999).

[67] S. Heinemeyer, W. Hollik, and G. Weiglein, Comput. Phys. Commun. 124, 76 (2000).

[68] A. Djouadi, J. Kalinowski, and M. Spira, Comput. Phys. Commun. 108, 56 (1998).

[69] A. Harel, DØ Internal Note 4919.

[70] T. Andeen et al., FERMILAB-TM-2365-E (2006).

[71] V. M. Abazov et al., Phys. Rev. Lett. 101, 071804 (2008). 
[72] M. Owen, A. Patwa, S. Söldner-Rembold, and W. C. Yang, DØ Internal Note 5728-CONF (2008).

[73] G. Bernardi, S. Choi, and K. Hanagaki, Dø Internal Note 5043 (2006).

[74] R. Hamberg, W. van Neerven, and T. Matsuura, Nucl. Phys. B 359 (1991).

[75] A. Martin, R. Roberts, W. Stirling, and R. Thorne, Phys. Lett. B 604, 61 (2004).

[76] T. Junk, Nucl.Instrum.Meth. A434, 435 (1999).

[77] W. Fisher, DØ Internal Notes 4975, 5309, and 5491, FERMILAB-TM2386-E (2006). 


\section{Appendix A}

\section{The Silicon Track Trigger}

The Silicon Track Trigger (STT) is a dedicated preprocessor within the Level 2 trigger system. Briefly mentioned in section 3.1.2 we now present a more detailed description.

\section{A.1 Introduction}

The overall concept of the STT is to match SMT barrel hits to L1CTT tracks to provide fast identification of high-impact parameter tracks. Such tracks often come from displaced vertices, a trademark of $b$ decays. By triggering on the presence of such tracks one can enhance the heavy flavor content of a data sample, important for Higgs searches, top physics, and $b$ physics.

Figure A.1 shows the STT pattern recognition concept. Tracks from L1CTT are used as seeds, or "roads". SMT hits within $\pm 2 \mathrm{~mm}$ of the road are considered for use in a fit. A "hit" is defined as a cluster of individual SMT channels that together pass a certain threshold (sec. A.2.2). There must be suitable hits in at least four of the five SMT layers for the fit to proceed. The $\left(r, \phi_{0}\right)$ hit positions go into a $\chi^{2}$ minimization function with the outputs being the track impact parameter $b$, radius of curvature $\kappa$, and $\phi_{0} . \phi_{0}$ is defined as the angle between the track's tangent line and the $x$-axis at the point of closest approach to the origin. The fit also corrects for the measured beam position from the previous data-taking run (the beam spot generally changes only slightly between runs) in order to get a more accurate impact parameter measurement.

The STT impact parameter resolution is a function of three main effects: the detector resolution, the Tevatron beam cross section size, and multiple scattering in the beam pipe and SMT. The detector resolution contributes about $19 \mu \mathrm{m}$ to the total resolution, the beam size about $35 \mu$, and the multiple 


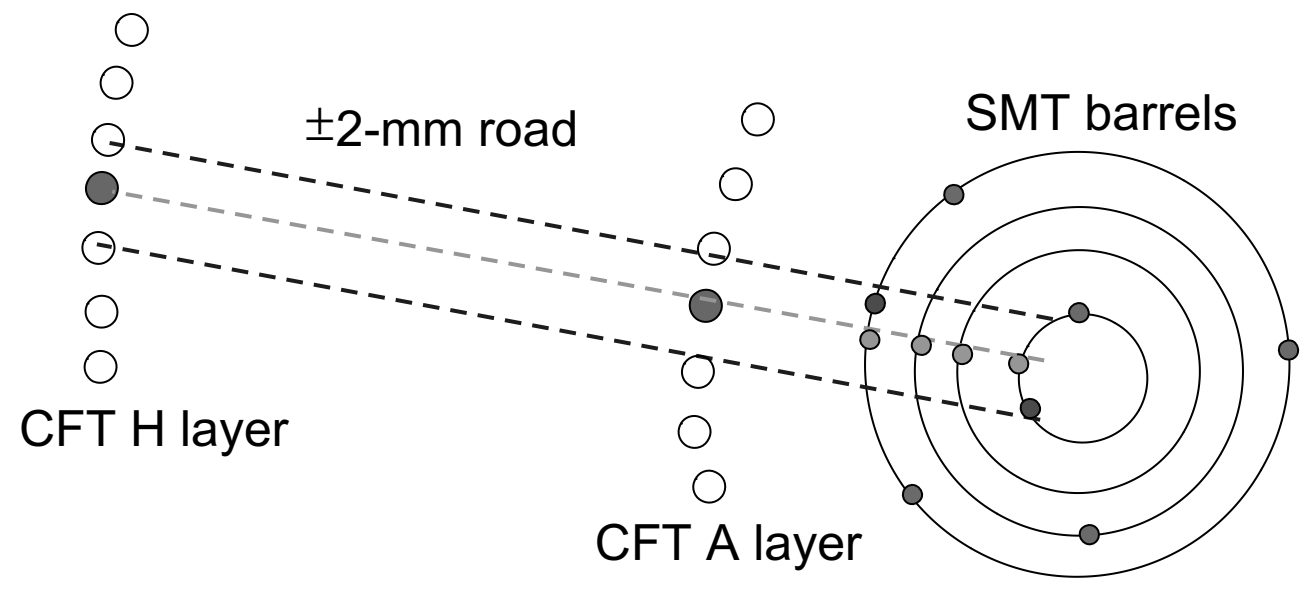

Figure A.1: STT pattern recognition. Clusters from SMT hits are associated to tracks from L1CTT; the STT then fits the SMT hits to a track by minimizing a $\chi^{2}$ function in $b, \kappa$, and $\phi$. [30].

scattering term is track $p_{T}$-dependent. The final total cross section is then

$$
\sigma_{b}=\sqrt{35^{2}+19^{2}+\left(\frac{54 G e V}{p_{T}}\right)^{2}} \mu \mathrm{m}
$$

where $p_{T}$ is the track $p_{T}$ in GeV. There are other small effects such as the $z$ position of the $p \bar{p}$ interaction and beam tilt, but these effects together are less than $5 \mu \mathrm{m}$.

\section{A.2 Technical Design}

There are six STT readout crates at D $\varnothing$ each covering approximately $1 / 6$ th of the total $\phi$ space. A crate consists of one Fiber Road Card (FRC), ten Silicon Trigger Cards (STCs), and two Track Fit Cards (TFCs). The L1 CTT tracks come in through the FRC, where they pass to the TFCs and STCs. Raw SMT hits go directly to the STCs, which form the SMT clusters from the raw hits and associate the clusters to the CTT roads. The output passes to the TFCs where the final hit/road association is done and the MT clusters are fit to tracks. Finally the TFC output passes to the L2CTT preprocessor (sec. 3.1.2) to take part in the trigger decision. On a L2 accept, the TFC output is also sent to Level 3 for bookkeeping and/or debugging purposes. Figure A.2 shows the flow of data through the STT. 


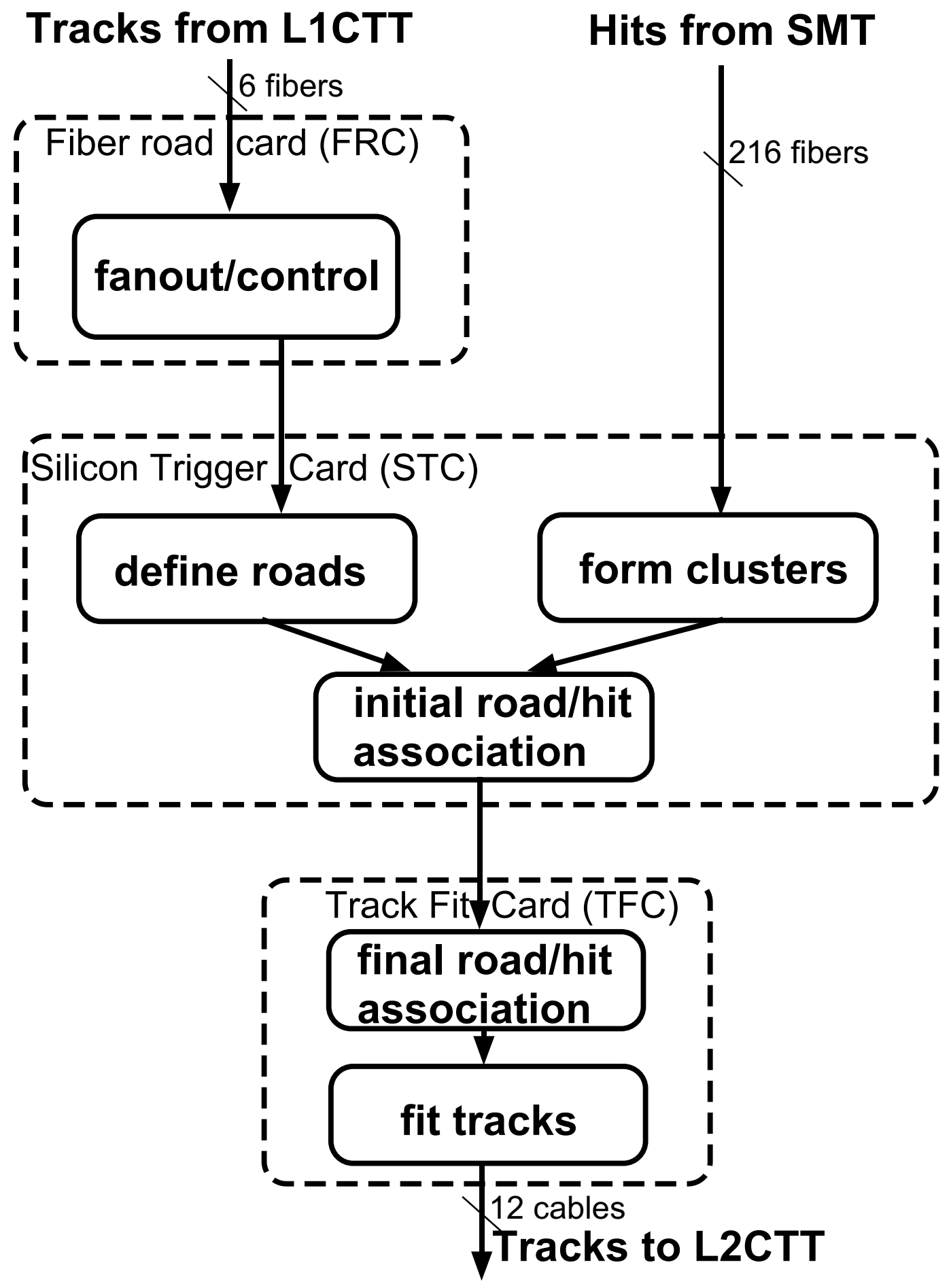

Figure A.2: Dataflow through the STT. The Level 3 data paths have been omitted [30]. 


\section{A.2.1 Fiber Road Card}

The Fiber Road Card (FRC) functions as the link between the crate and the rest of the trigger system, executing the tasks sent by the trigger framework such as resets, initializations, and sending a copy of the STT output to Level 3 on L2 accepts. It also receives the L1CTT tracks in events where there is an L1 trigger accept, and sends them to the appropriate STCs and TFC(s) based on their $\phi$ position. It also serves a conflict manager between the single board computer (SBC) in the crate and the in-crate CPU used for initialization.

Figure A.3 is a block diagram of the FRC. Communication with the trigger framework is handled through the Serial Command Link formatter (SCLF). The L1CTT tracks come in on a VME Transition Module (VTM) where the Trigger/Road data formatter (TRDF) receives the information and combines it with data from the SCLF. After a check for a bunch crossing (BX) number match between the two data sources the TRDF packages the CTT and needed SCLF data into a block consisting of between six and fifty-four 32-bit words, depending on the number of CTT tracks. Only the first 46 tracks (sorted in decreasing $p_{T}$ ) from the CTT are sent on in each of the six crates. The data block then goes to the first two of three PCI buses where Link Transmitter Boards (LTBs) send the block to the STC and TFC cards. The Buffer Manager (BM) in each FRC is responsible for generating error signals, passing initialization commands and monitoring data collection requests to the in-crate CPU, buffering the L3 data for transmission, and scheduling VME accesses to accomplish these tasks.

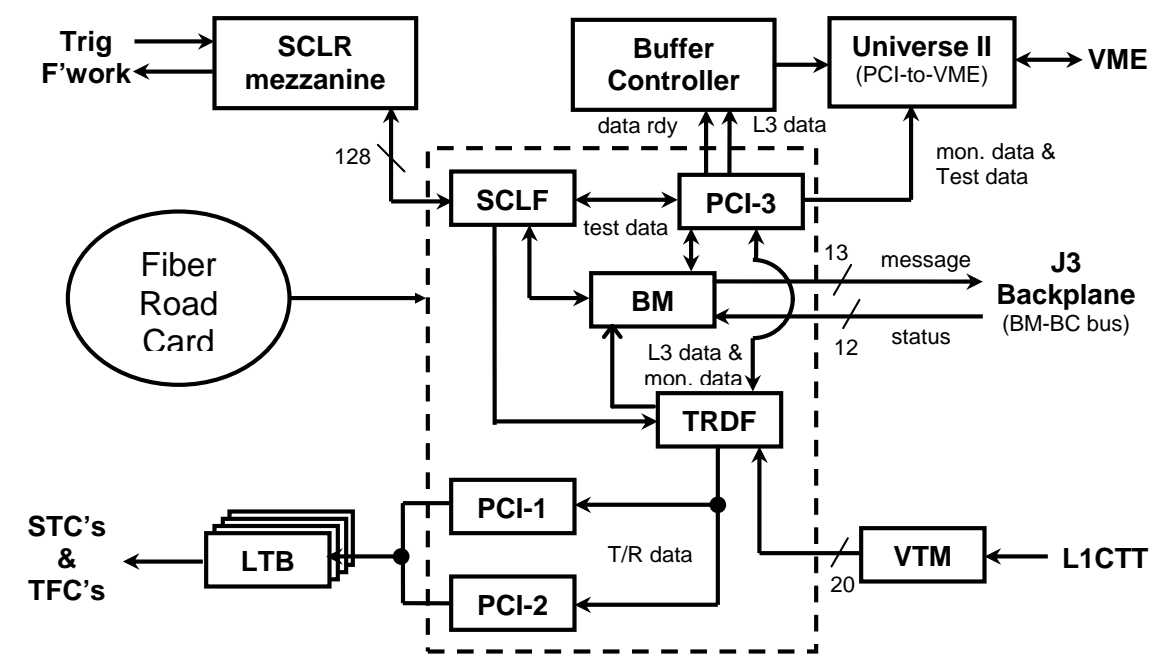

Figure A.3: Block diagram of the Fiber Road Card (FRC). The dashed rectangle denotes the on-board FRC components [30]. 


\section{A.2.2 Silicon Trigger Card}

The STC receives SMT data over the HP G-link protocol into VTMs via optical fibers, and the L1CTT data from the FRC. Its tasks include performing pedestal subtraction on the SMT channels, removing dead or noisy channels, clustering the channels together, and finally associating those clusters to the roads defined by the L1CTT tracks. Figure A.4 shows a block diagram of the STC.

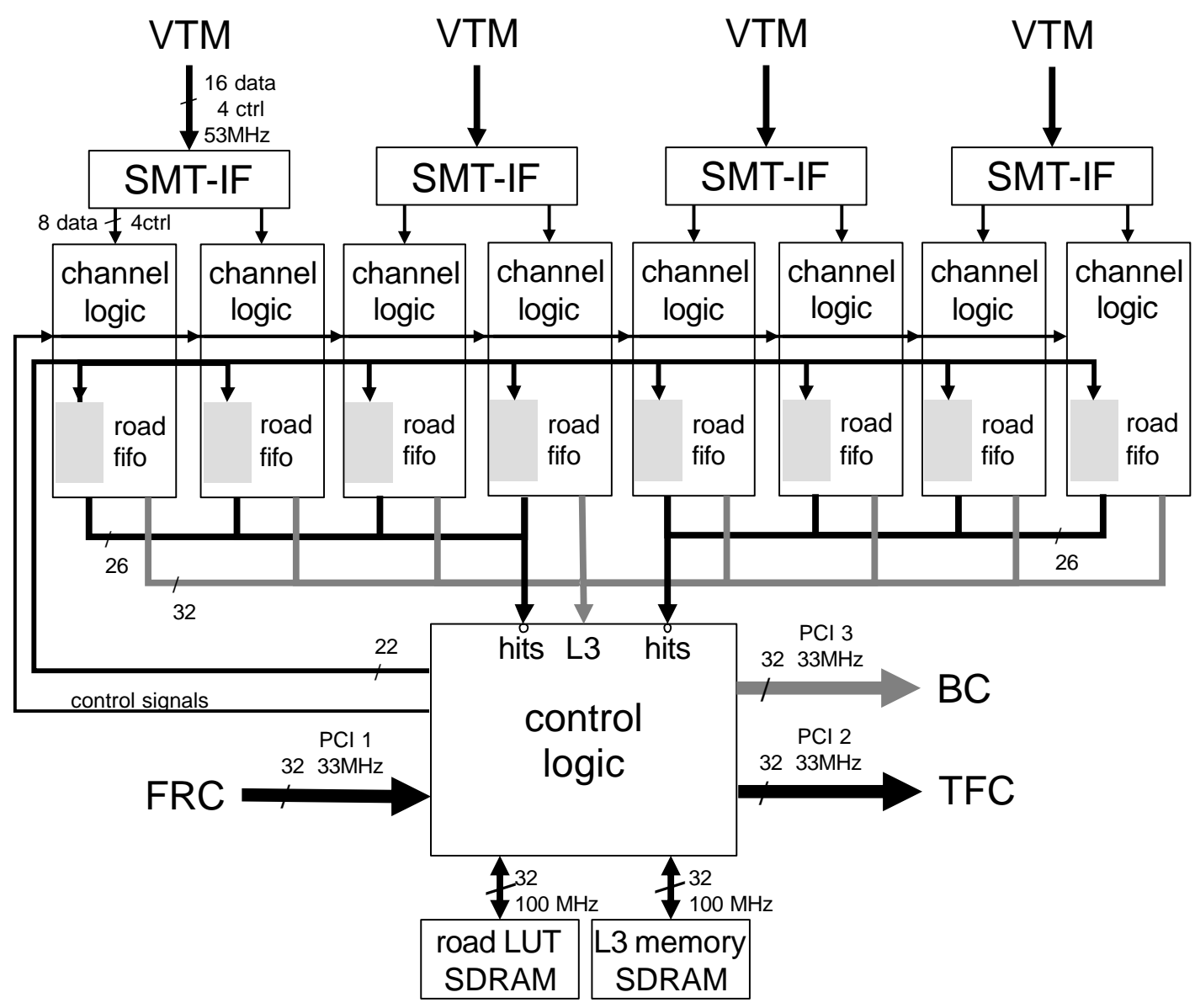

Figure A.4: Block diagram of the Silicon Trigger Card (STC)[30].

Each of the ten STCs within a crate receives SMT input data from a unique VTM over four optical fibers, with each fiber carrying data for two detector elements. The data go into one of four SMT input FIFOs, or SMT-IFs, as shown. There are eight bits for each detector element, along with four control bits; thus there are 20 bits per fiber. The data from each SMT-IF are split into two streams of eight SMT plus four control bits, and go into channel logic 
blocks. The channel logic blocks perform the pedestal subtraction, clustering, and hit-road association. The clusters associated with roads are thereafter called "hits". The control logic block is the heart of the STC; it manages the overall event processing, issuing control signals to the channel logic and the PCI interfaces. The PCI interfaces are where the input and output pass thought; the first PCI interface receives the L1CTT tracks from the FRC; the second takes the hits from the channel logic and transfers them to the TFC, while the third receives downloadable parameters (such as SMT channel pedestals) and transfers the L3 data to the Buffer Controller for output to L3.

\section{Clustering and Hit-road Association}

The core functions of the STC, the clustering and hit-road association, occur in the channel logic. The first thing that happens to the SMT data received by the SMT-IFs is pedestal subtraction. For each SMT channel the STC subtracts a certain number of ADC counts, known as the pedestal, to correct for noise effects. The pedestal for each channel is a downloadable parameter that is loaded at initialization time. The pedestal-subtracted channels are also checked against a list of known bad channels (also downloaded at initialization); channels on this list have their ADC counts set to 0, effectively eliminating them. The channels then pass through a centroid-finding algorithm that compares neighboring strips and finds a centroid. The centroid position is then used as the cluster position. The total pulse height under the centroid is a measure of the energy deposited in the SMT and also goes to the TFC, but the dynamic range is scaled to fit into three bits.

All clusters' centroid positions get compared to all roads to associate the roads to clusters within $2 \mathrm{~mm}$ of the road. The comparison works by looking at two 11-bit addresses the correspond to the chip and strip numbers of the road edges as defined by that particular CTT track. If the centroid position, encoded in a 13-bit address, has a a leading 11 bits that lie within the two two addresses for the road, then that cluster gets associated to the road and is then called a "hit." It is possible for a centroid to be associated with more than one road, and for roads to have multiple hits within each SMT layer. The TFC performs the final hit-road association. the associated hits then pass to the TFC for final association and track fitting.

\section{A.2.3 Track Fit Card}

Each STT crate has two Track Fit Cards (TFCs). The TFCs receive their input from the FRC and seven of the 10 STCs. Due to the detector geometry, there are three STCs per crate that send data to only the first TFC, three that send 
only to the second, and four that send to both. The TFC performs filtering on the initial hit/road association and chooses the final associations, fits the clusters, and outputs the result to L2CTT for use in the trigger decision.

Figure A.5 shows the data flow through the various parts of the TFC. The input data from the STCs and the FRC comes in on the PCI-1 bus. The data from the FRC are an exact copy of the L1CTT input tracks, with header and trailer data added. The STC data include the centroid for each hit, the 3-bit pulse height, and an index referencing the CTT track to which the hit has been associated. The index has the same order as the L1CTT input tracks. The STC and FRC are initially held in Input Dual-Port Memory (IDPM), with each road and its associated clusters occupying consecutive memory locations. As shown in the figure the IDPM is has two independent banks, one holding odd-numbered roads and the other holding even-numbered roads. The entire event is held in the IDPM until all STC clusters have been read in. During the read-in, the SMT cluster centroid information is converted into an effective $r, \phi$ position for use in the fitting by means of a coordinate conversion lookup table (CCLUT in figure A.5.) This position then gets sent for fitting. The fits take place inside Digital Signal Processors (DSPs), with four DSPs assigned to each IDPM bank as shown in figure A.6. We describe the fitting procedure in detail below. As fits are completed they go to Output Dual-Port Memory (ODPM) until all fits in an events are done. Then the output fits pass to the PCI-2 bus where they are formatted with $\mathrm{D} \varnothing$ standard headers and trailers attached. The bus also synchronizes the event information, preserving the event time-ordering, and then the fits pass out over the Hotlink Transmitter, going to L2CTT.

\section{Track Fitting}

The first step in the track fitting called final filtering, which finalizes the cluster/road association; only one cluster per SMT layer is chosen. In the first part of the final filtering the road width narrows from $\pm 2 \mathrm{~mm}$ to $\pm 1 \mathrm{~mm}$, eliminating clusters outside of $1 \mathrm{~mm}$. Then, starting from the outermost possible layer, an algorithm searches for combination of hits consistent with a straight line in the $r-z$ plane. Effectively, this means that the hits selected in each layer must all be within the same SMT barrel, or if there is a change, there can be only one change over all layers, and this change must not be more than one barrel. Clusters surviving these constraints pass to the second stage of the final filtering, which measures the $r_{i} \delta \phi$ distance between each cluster and the CTT track. $r_{i} \delta \phi$ is defined as

$$
r_{i} \delta \phi=\mid r_{i}\left(\phi_{i} \phi_{0 C T T}-\kappa_{C T T} \dot{r}_{i}^{2}-b_{C T T} \mid\right.
$$




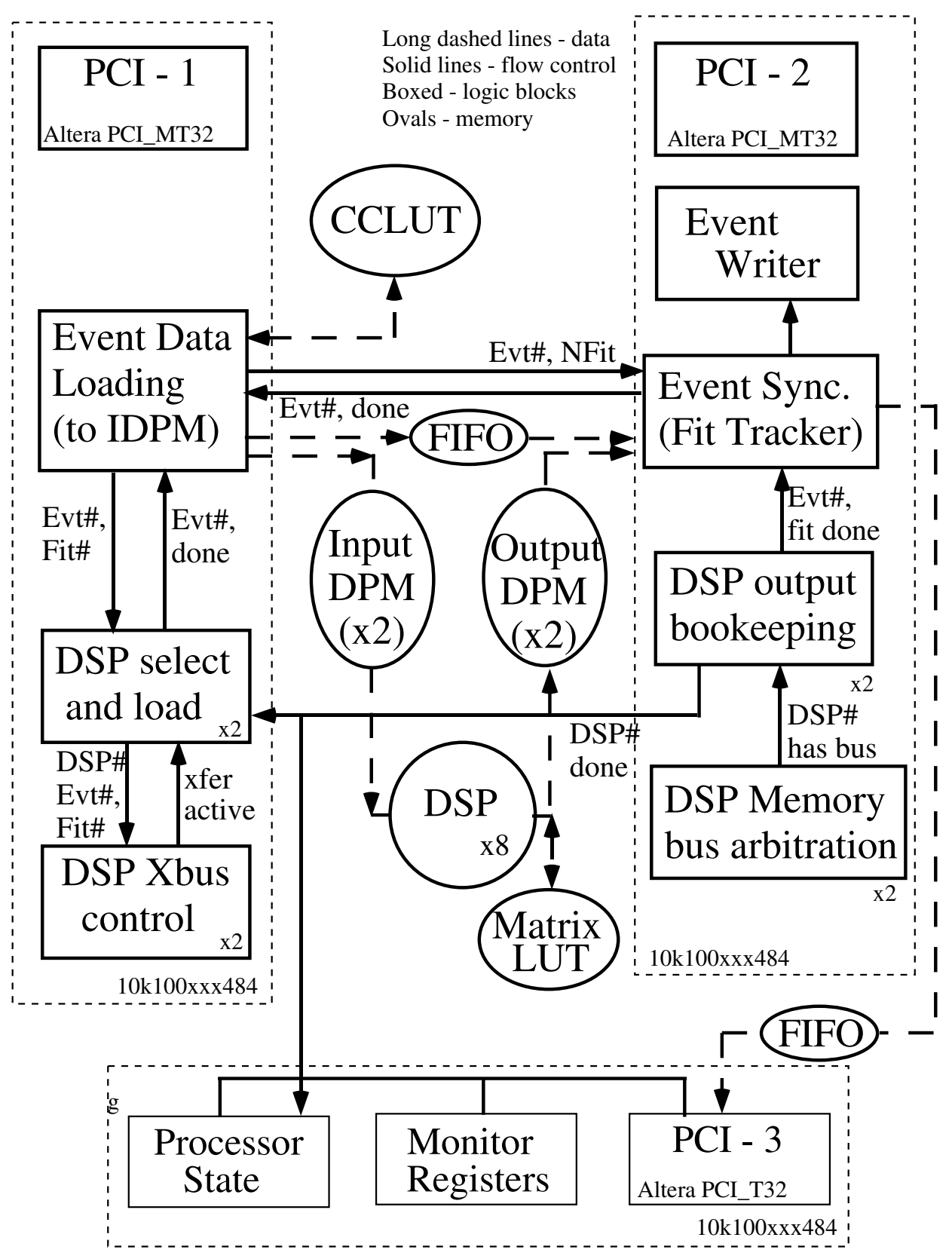

Figure A.5: Block diagram of the Silicon Trigger Card (STC)[30]. 


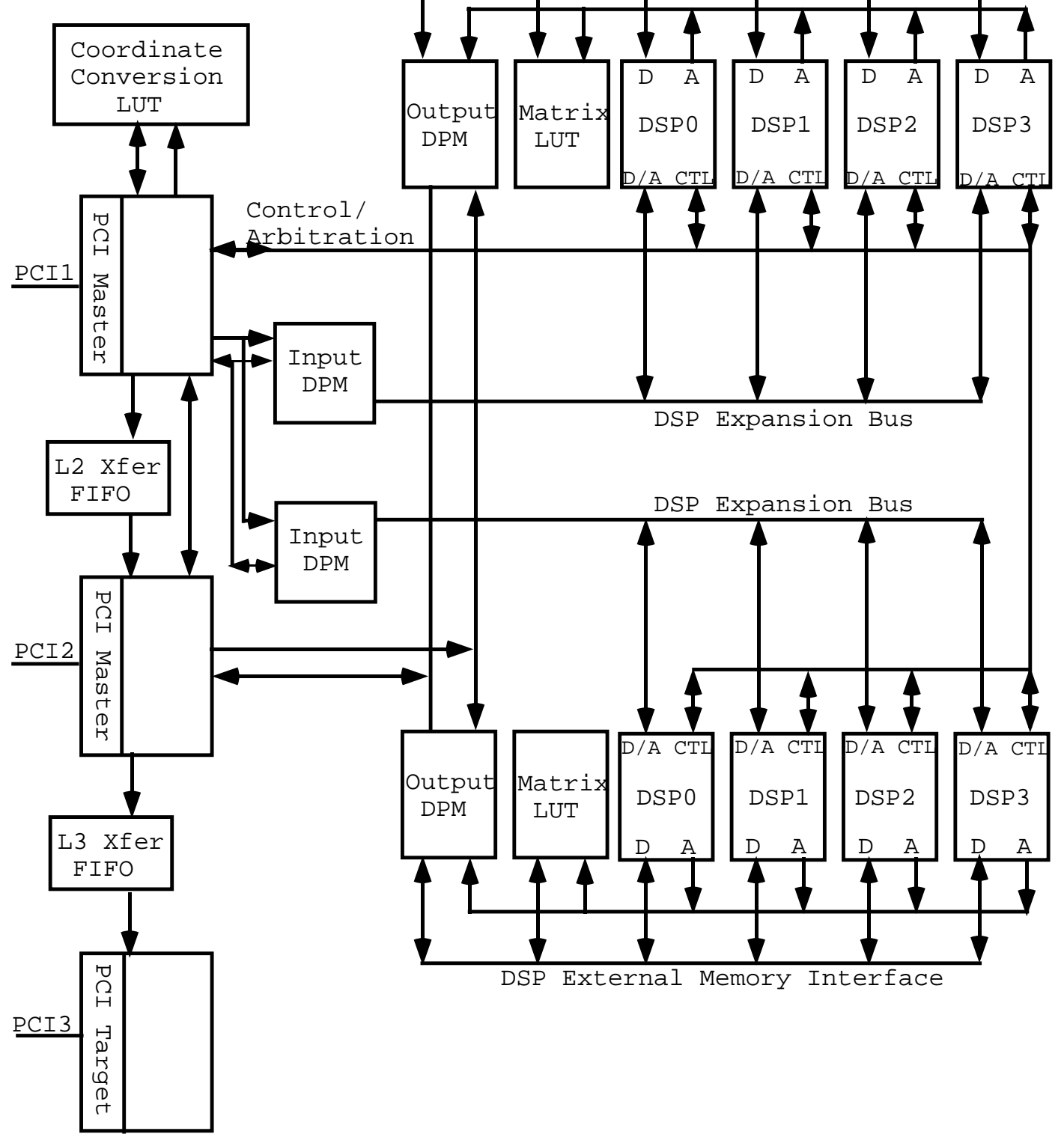

Figure A.6: Block diagram of the Track Fit Card (TFC)[30]. 
where $r_{i}$ and $\phi_{i}$ are the $r$ and $\phi$ positions of the $i$-th cluster, and $\phi_{0 C T T}$ and $\kappa_{C T T}$ are the track azimuthal angle and curvature relative to the measured beam position. $b_{C T T}$ is the CTT track impact parameter measured with respect to the detector origin. In each SMT layer, the chosen cluster is the one that has the smallest $r_{i} \delta \phi$ value.

After the final filtering there are SMT hits from either four or five layers. We use these, plus the CTT track $\left(r, \phi_{0}\right)$ position where it crosses the CFT A layer and $\mathrm{H}$ layer in the final track fitting. We assume the track has a circular trajectory in the $r, \phi$ plane near the origin. One needs three parameters to define such a circular trajectory; they are the signed impact parameter $b$, the track $\phi_{0}$, and curvature $\kappa$. We approximate a circle close to the origin by the expression

$$
\phi(r)=\frac{b}{r}+\kappa r+\phi_{0}
$$

We base the impact parameter sign on whether the origin is inside or outside this circle. We say the product $b \kappa$ is positive when the origin is inside the circle, and negative when it is outside the circle.

We can use equation A.3 to describe the fitted track trajectory. To obtain the track parameters we minimize the $\chi^{2}$ function

$$
\chi^{2}=\sum_{\text {clusters }}\left[\frac{r_{i} \phi_{i}-r_{i} \phi\left(r_{i}\right)}{\sigma_{i}^{2}}\right]^{2}
$$

A linear least-squares minimization yields the equation for the three parameters of interest:

$$
\begin{aligned}
b & =\sum_{j} M_{1 j} \Phi_{j} \\
\phi_{0} & =\sum_{j} M_{2 j} \Phi_{j} \\
\kappa & =\sum_{j} M_{3 j} \Phi_{j}
\end{aligned}
$$

with $j=1,2,3, \Phi_{n}=\sum_{k} r_{k}^{n} \phi_{k} / \sigma_{k}^{2}\left(k=1, \ldots, N_{\text {points }}\right)$. All of the fitting steps take place in the DSPs. However, the only have support for integer adds and multiplies; they cannot do floating-point calculations. In addition, the DSP time budget is $100 \mu$ s per fit so it is important to have as fast a procedure as possible. Thus calculating the parameters from these equations is not feasible as it involves calculating the $M_{i j}$ matrices. If, however, we "re-center" the coordinate system such that the $\phi$ angles are expressed as differences from the 
$\phi$ value of one of the points $\left(\phi_{1}\right)$ we can rewrite Eqs. A.5 through A.7 as

$$
\begin{aligned}
b & =\sum_{k} M \prime_{1 k} \delta \phi_{k} \\
\phi_{0} & =\sum_{k} M \prime_{2 k} \delta \phi_{k} \\
\kappa & =\sum_{k} M \prime_{3 k} \delta \phi_{k}
\end{aligned}
$$

with $\delta \phi_{k} \equiv \phi_{k}-\phi_{1}$. Now the matrix elements and $\delta \phi_{k}$ values can be rescaled into 16-bit signed integers with ease. Additionally, we divide the $\phi$ space into 1,440 slices, and we use the same matrix values for each slice. This gives acceptable precision. Further, there are actually several thousand separate matrices for each $\phi$ slice to take into account the different possibilities of barrel combinations and whether or not four or five layers go into the fit. All of the matrices are computed offline and stored in a Matrix Lookup Table (Matrix LUT in figures A.5 and A.6.) Finally, then, the tracks fitting works as follows: for each $(r, \phi)$ hit position, after $\phi$ conversion, the appropriate integer multiplications take place within the DSP and the Matrix LUT is accessed to obtain the appropriate elements. Through the use of an Inverse Matrix LUT, one gets back the fitted track $b, \kappa$, and $\phi_{0}$.

The final chisq value of the fit is a measure of quality. Since the approximations made in the fit neglect multiple scattering, we scale the fit $\chi^{2}$ by the

following factor $1 / \sqrt{4+\left(8 / p_{T}\right)^{2}}$. The $\chi^{2}$ is then scaled to an integer ranging from 0 to 31 . If a fit contains hits from all five layers but the chisq is 31, i.e. a bad fit, we drop the SMT hit with the highest individual contribution to the $\chi^{2}$ and re-fit with the remaining four SMT hits. This is called "two-pass fitting." Five-layer fits with chisq $<31$ are always preferred over four layer fits, however, even if a four-layer fit might have a lower $\chi^{2}$.

\section{A.3 Performance}

To measure the STT's physics performance we match tracks from the STT L3 output to reconstructed offline tracks (RECO tracks), using the reconstructed tracks as the standard. If the STT is performing well then there should be an STT track overlapping the reconstructed track when the reconstructed track is within the STT fiducial acceptance. Since RECO tracks are not required to have SMT hits or lie in a certain $\eta$ range, we place the following cuts on RECO tracks before considering them as "good", to match the STT design 
constraints:

- $p_{T}>1.5 \mathrm{GeV}$

- $\mid$ eta $\mid<1.6$, i.e. within the CTT acceptance

- $\operatorname{track} \chi^{2}<4$

- hits in $\geq 7$ CFT axial layers

- hits in $\geq 4$ SMT layers

- overlap with L1CTT track

These cuts ensure that the reco track is within the STT's acceptance and that conditions exist such that an STT track can be found, namely, a CTT track and hits in at least four SMT layers. They also protect against fake RECO tracks that would artificially lower the measured efficiency. Good STT tracks are those STT tracks output to L3 that have $p_{T}>1.5 \mathrm{GeV}$ and a fit $\chi^{2}$ passing a chosen cut. We can then define the efficiency $\epsilon$ as

$$
\epsilon=\frac{N_{S T T-R E C O \text { match }}}{N_{\text {good } R E C O}}
$$

and conversely the purity $P$ as

$$
P=\frac{N_{S T T-R E C O \text { match }}}{N_{\text {good } S T T}}
$$

the match criterion is a $\chi^{2}$ consisting of the tracks' impact parameter (measured with respect to the beam position), $\kappa$, and $\phi$ values. A low $\chi^{2}$ implies that the tracks are similar. We measure the efficiency and purity in two different samples: in $Z \rightarrow \mu \mu$ events where we have clean, high $p_{T}$ tracks, and in a sample dominated by multijet events, consisting of mostly low- $p_{T}$ tracks.

\section{A.3.1 Performance in $Z \rightarrow \mu \mu$ events}

$Z \rightarrow \mu \mu$ events provide a good sample in which to measure the STT efficiency on high- $p_{T}$ tracks. These are very often clean tracks with little else to degrade the resolution, so we should see a high efficiency here. To measure the efficiency in this sample we only consider the two RECO tracks matched to the two muons identified as the $Z$ daughters. To get only these two tracks we impose additional requirements on the RECO tracks on top of those in section A.3. We raise the $p_{T}$ threshold to $15 \mathrm{GeV}$, and require that the invariant mass of 
the two tracks is consistent with the $Z$ mass, namely $76<M_{\mu \mu}<106 \mathrm{GeV}$. We also modify the good STT track definition by raising the $p_{T}$ threshold to $15 \mathrm{GeV}$ as well. Figure A.7 shows the STT efficiency as a function of the STT track fit $\chi^{2}$. Here the track fit $\chi^{2}$ is the integer fit $\chi^{2}$ value divided by 2 . We see that for a $\chi^{2}$ cut of 5 , a typical value used in a trigger, the STT is $85 \%$ efficient, and $90 \%$ is one does not cut on the fit $\chi^{2}$.

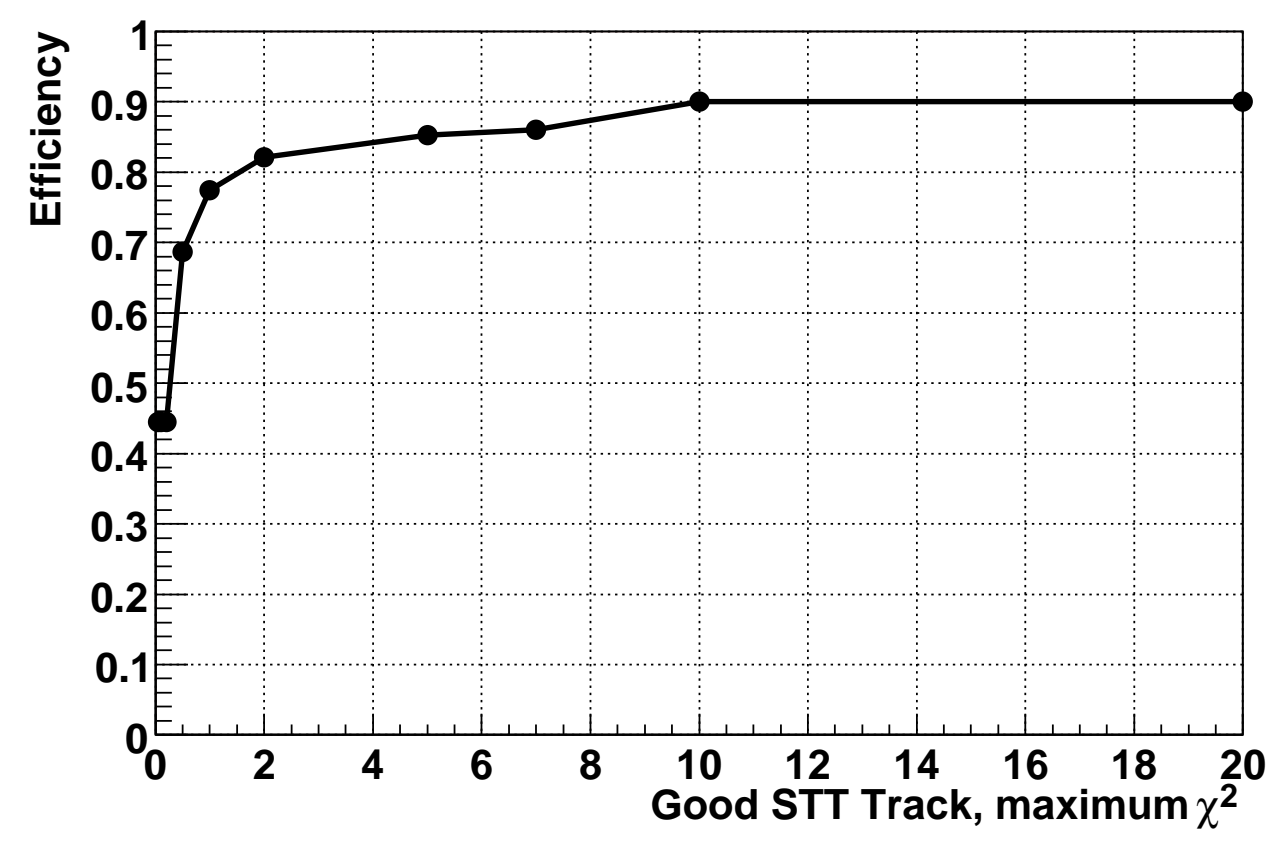

Figure A.7: Efficiency for a good STT track to be matched to a good RECO track as a function of the STT track fit $\chi^{2}[30]$.

\section{A.3.2 Performance in multijet events}

It is also instructive to measure the efficiency in multijet events, dominated by low $p_{T}$ tracks. In this case we define good STT and reco tracks according to the standard definitions given above. Figure A.8 shows the efficiency vs. purity for several different cut values of the STT track $\chi^{2}$. The data sample is a typical run with no detector problem and a low to intermediate instantaneous luminosity $\left(\approx 6 \times 10^{31} \mathrm{~cm}^{-2} \mathrm{~s}^{-1}\right.$.) We see that for a scaled $\chi 2$ cut value of 5 , the STT is $64 \%$ efficient with $87 \%$ purity. The efficiency increases to $70 \%$ with $84 \%$ purity for no $\chi^{2}$ cut. 


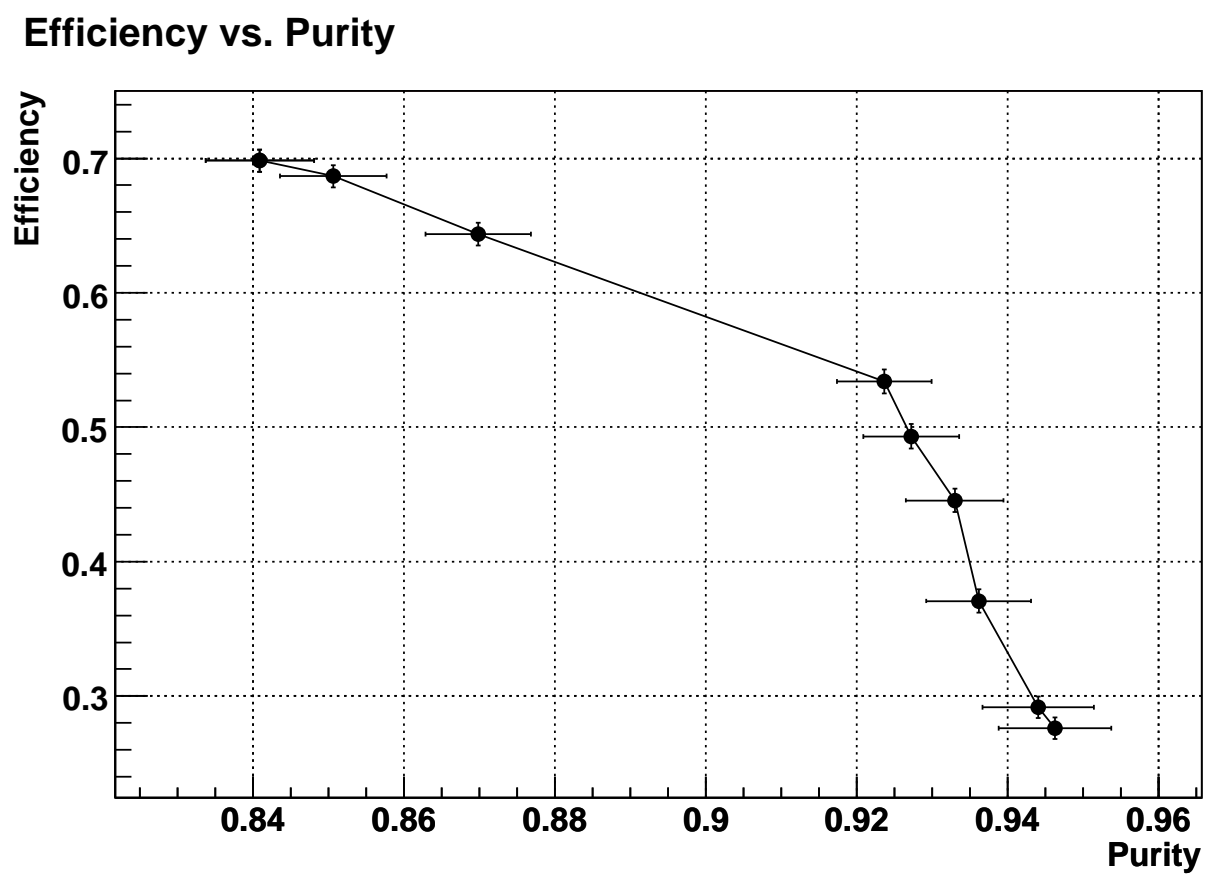

Figure A.8: Efficiency for a good STT track to be matched to a good RECO track as a function of the STT track fit. The different point represent different values of the STT track $\chi^{2}$. Looser cuts have low purity and high efficiency, while tighter cuts have lower efficiency but higher purity. 


\section{A.3.3 Use in trigger design}

The STT has several possible uses in the trigger. In some triggers one simply requires an STT track without regard to fit quality or impact parameter. In this case the idea is to use the silicon information to improve the momentum resolution on the track. In other triggers, there is a cut on the STT track's impact parameter significance. This is an excellent way to increase the heavy flavor content of the triggered sample. One such example in the D $\varnothing$ trigger list is a dedicated multijet trigger. At Level 2, the trigger requirement is an OR of four separate requirements. Two of the four terms require an STT track with impact parameter significance $\geq 3, \chi^{2}<5.5$, and $p_{T}>5 \mathrm{GeV}$. By requiring an STT track in the trigger, one can enhance the $b$-jet content of the sample, and avoid cutting on other topological variables involving the jets that lower efficiency. 


\section{Appendix B}

\section{Data/Monte Carlo Comparison Plots}

In this appendix we show a number of plots comparing data and Monte Carlo for different kinematic and topological variables.

\begin{tabular}{|c|c|}
\hline \multicolumn{2}{|c|}{ Key for Plots } \\
\hline - & Data \\
\hline & $\overline{\mathrm{Z}}+\mathrm{HF}+\mathrm{j} \rightarrow \tau \tau+\mathrm{HF}+\mathrm{j}$ \\
\hline & $\mathrm{Z}+\mathrm{j} \rightarrow \tau \tau+\mathrm{j}$ \\
\hline & QCD \\
\hline & $\mathrm{W}+\mathrm{HF}+\mathrm{j} \rightarrow \mathrm{l}+\mathrm{HF}+\mathrm{j}$ \\
\hline & $\mathrm{W}+\mathrm{j} \rightarrow \mathrm{lv}+\mathrm{j}$ \\
\hline & $\mathrm{Z}+\mathrm{HF}+\mathrm{j} \rightarrow \mu \mu+\mathrm{HF}+\mathrm{j}$ \\
\hline & $Z+j \rightarrow \mu \mu+j$ \\
\hline & Single top \\
\hline & WW/WZ \\
\hline & 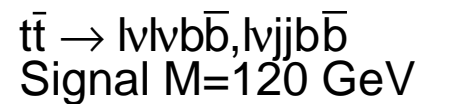 \\
\hline
\end{tabular}

Figure B.1: Expanded view of the legend used for kinematic and topological variable plots in Appendix B and Chapter 6. Here "HF" means heavy flavor, $b \bar{b}$ or $c \bar{c}$ pairs, and "j" means additional light quark or gluon jets. 


\section{B.1 Data/Monte Carlo Plots Before $b$-tagging}
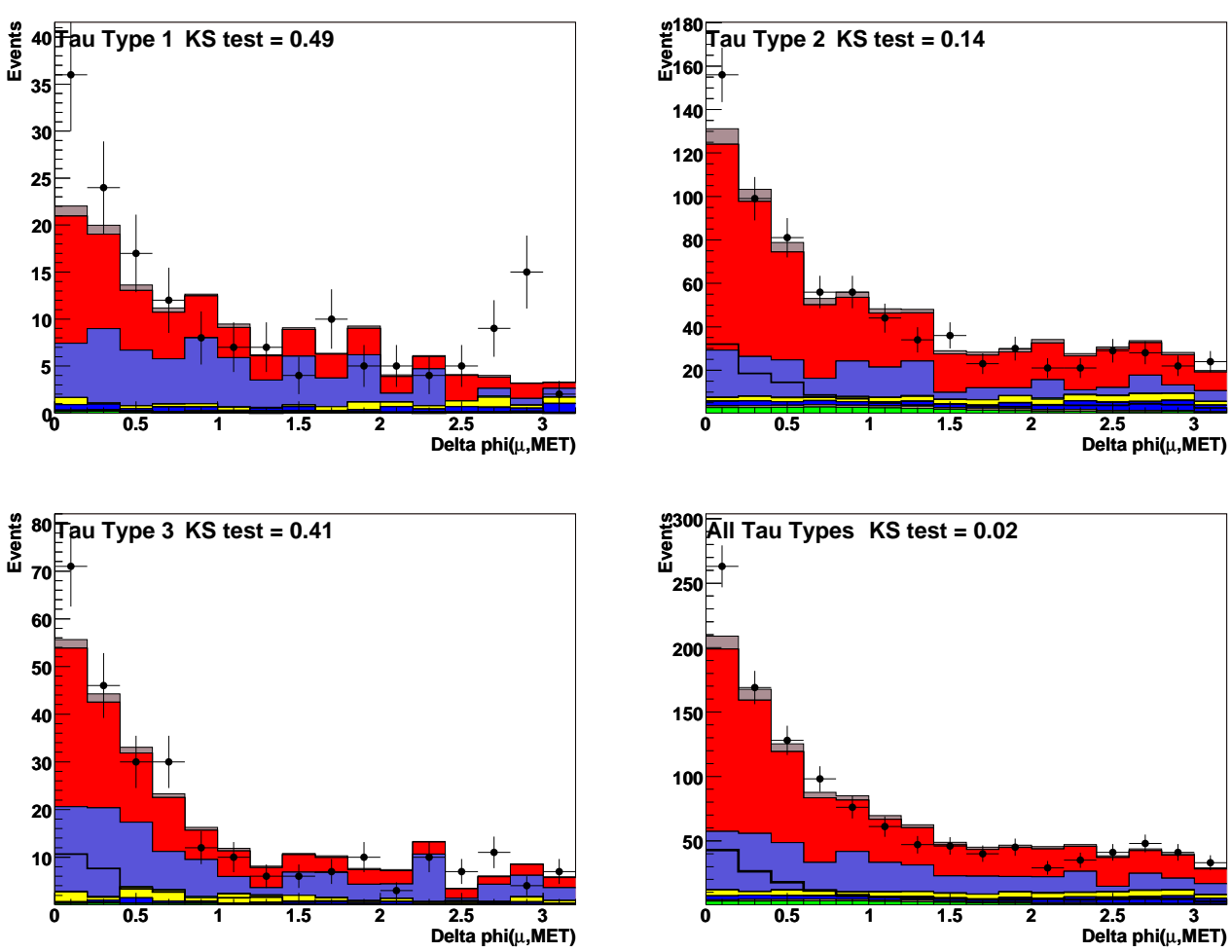

Figure B.2: $\Delta \phi\left(\mu, \mathbb{E}_{T}\right)$ before $b$-tagging for: Type 1 taus (upper left), Type 2 (upper right), Type 3 (lower left), and all types (lower right). 

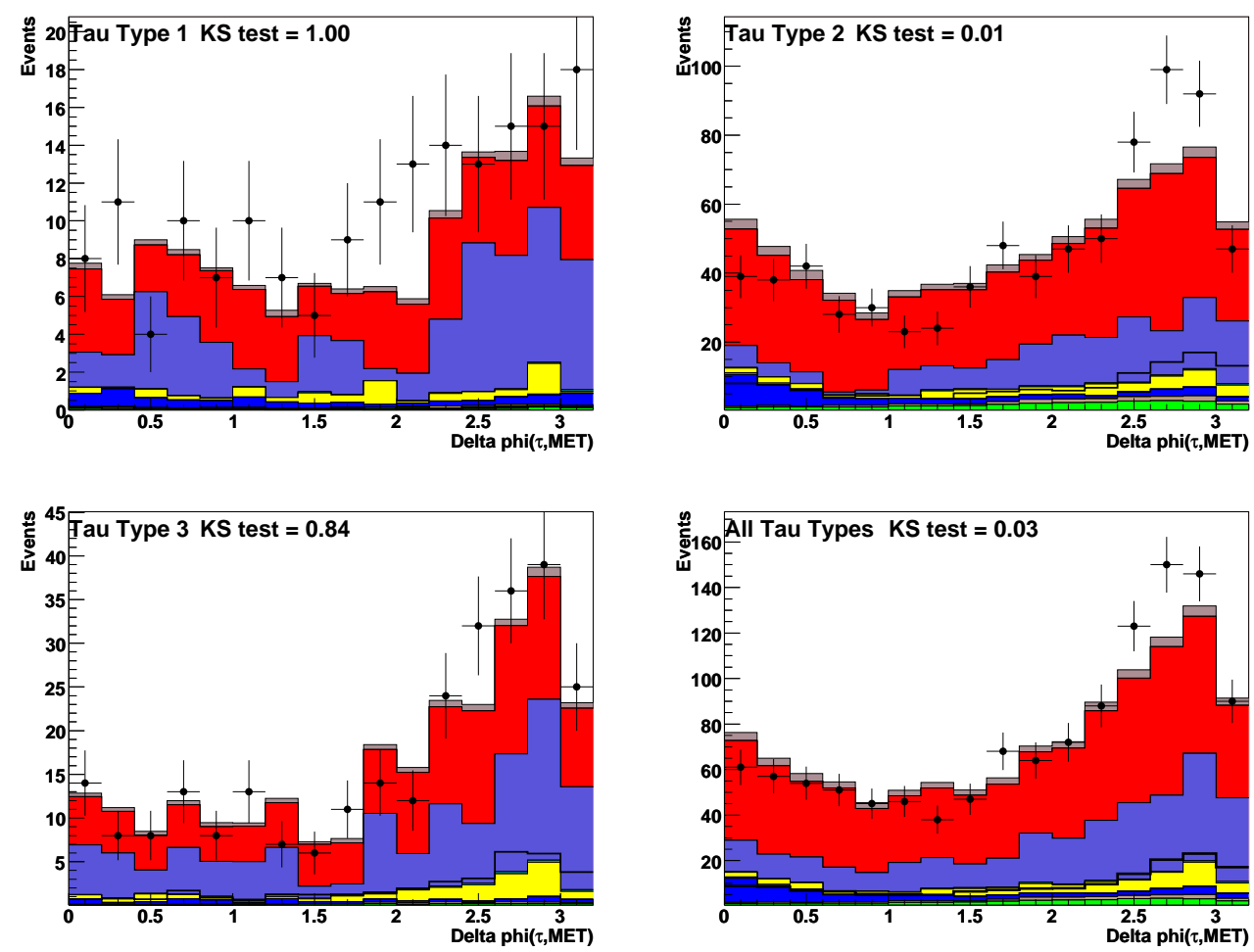

Figure B.3: $\Delta \phi\left(\tau, \mathbb{E}_{T}\right)$ before $b$-tagging for: Type 1 taus (upper left), Type 2 (upper right), Type 3 (lower left), and all types (lower right). 

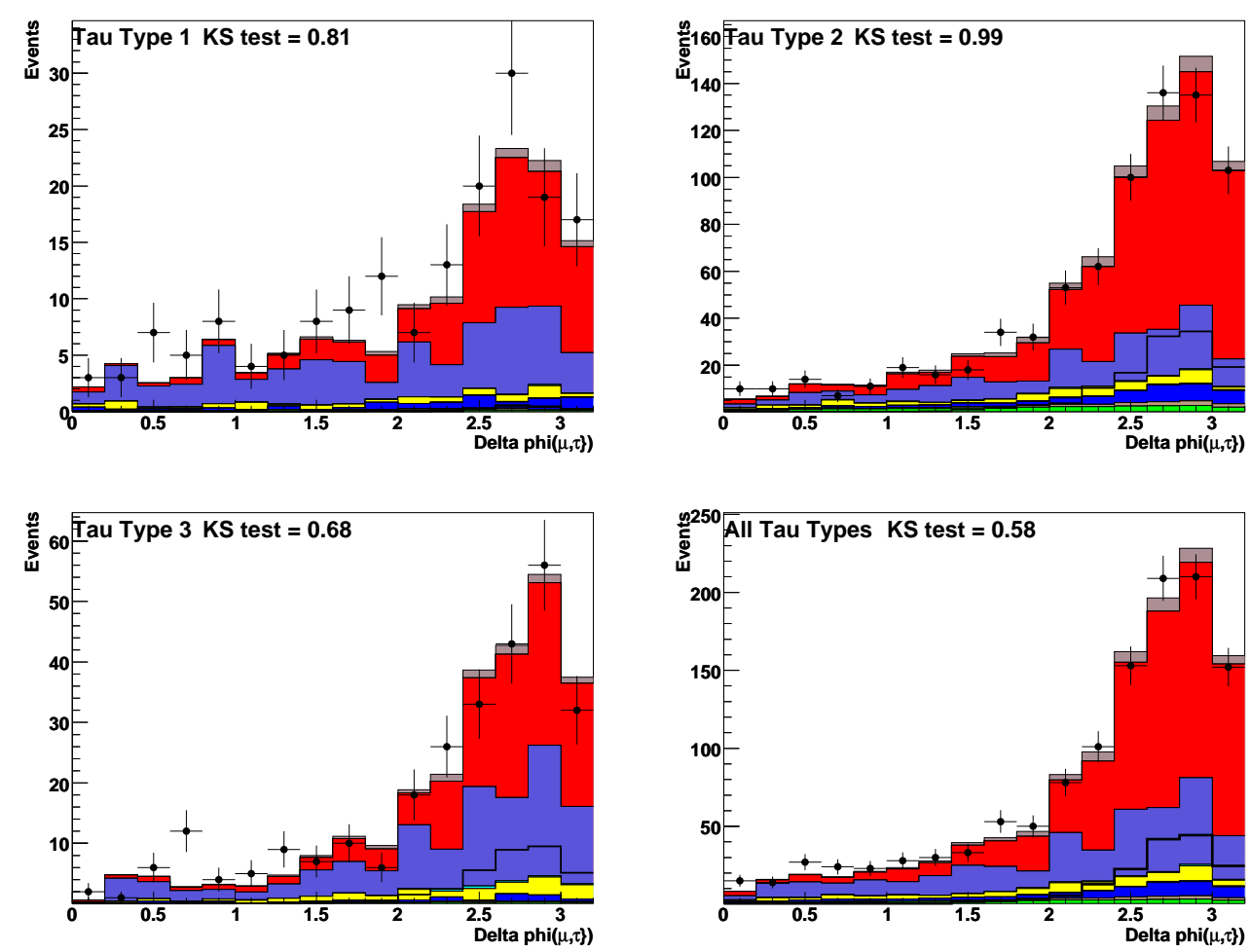

Figure B.4: $\Delta \phi(\mu, \tau)$ before $b$-tagging for: Type 1 taus (upper left), Type 2 (upper right), Type 3 (lower left), and all types (lower right). 

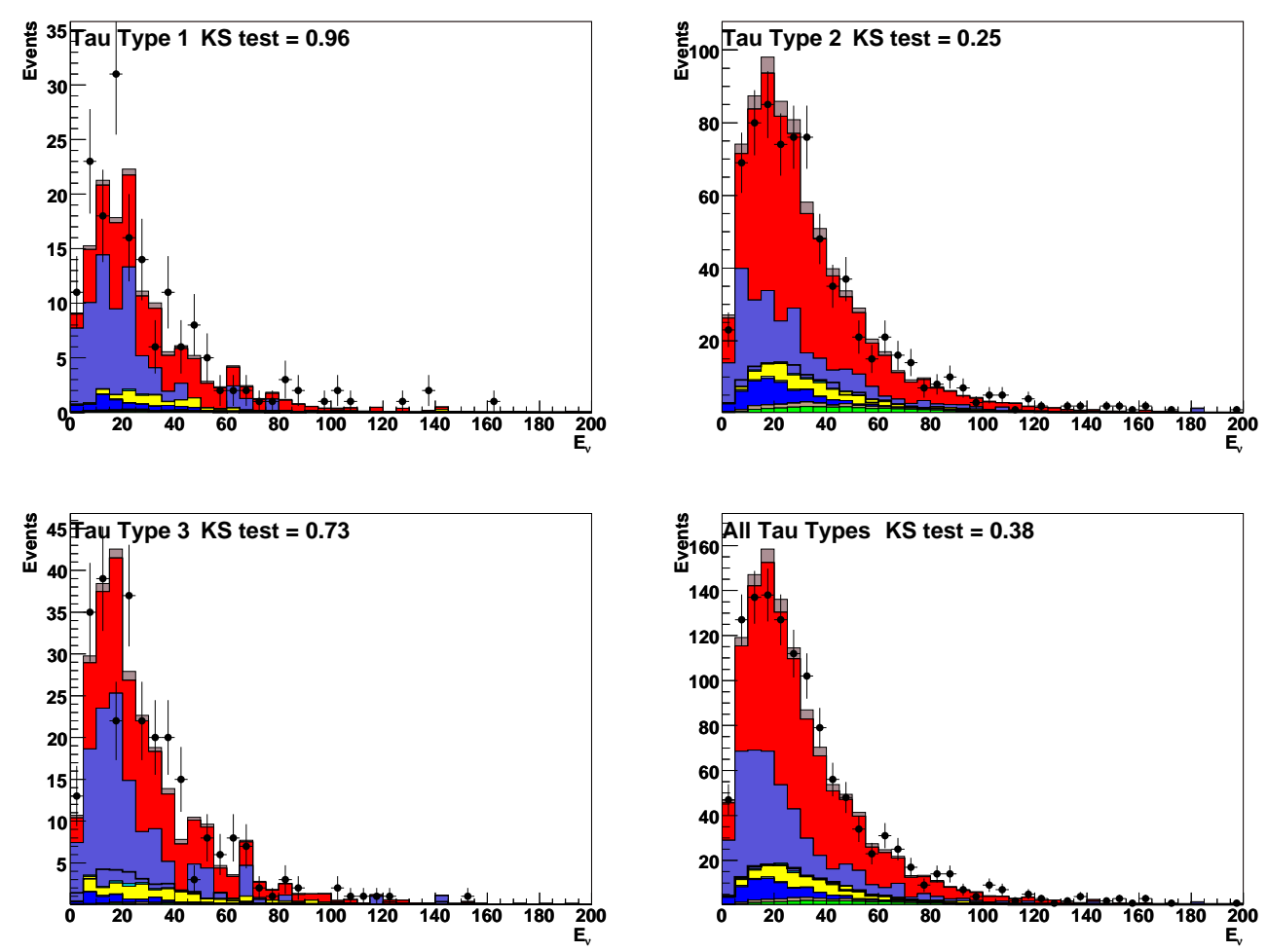

Figure B.5: The Enu (or $E_{\nu}$ ) variable, $E_{\nu} \equiv \mathbb{E}_{T} E_{\mu} / p_{T \mu}$ before $b$-tagging for: Type 1 taus (upper left), Type 2 (upper right), Type 3 (lower left), and all types (lower right). 

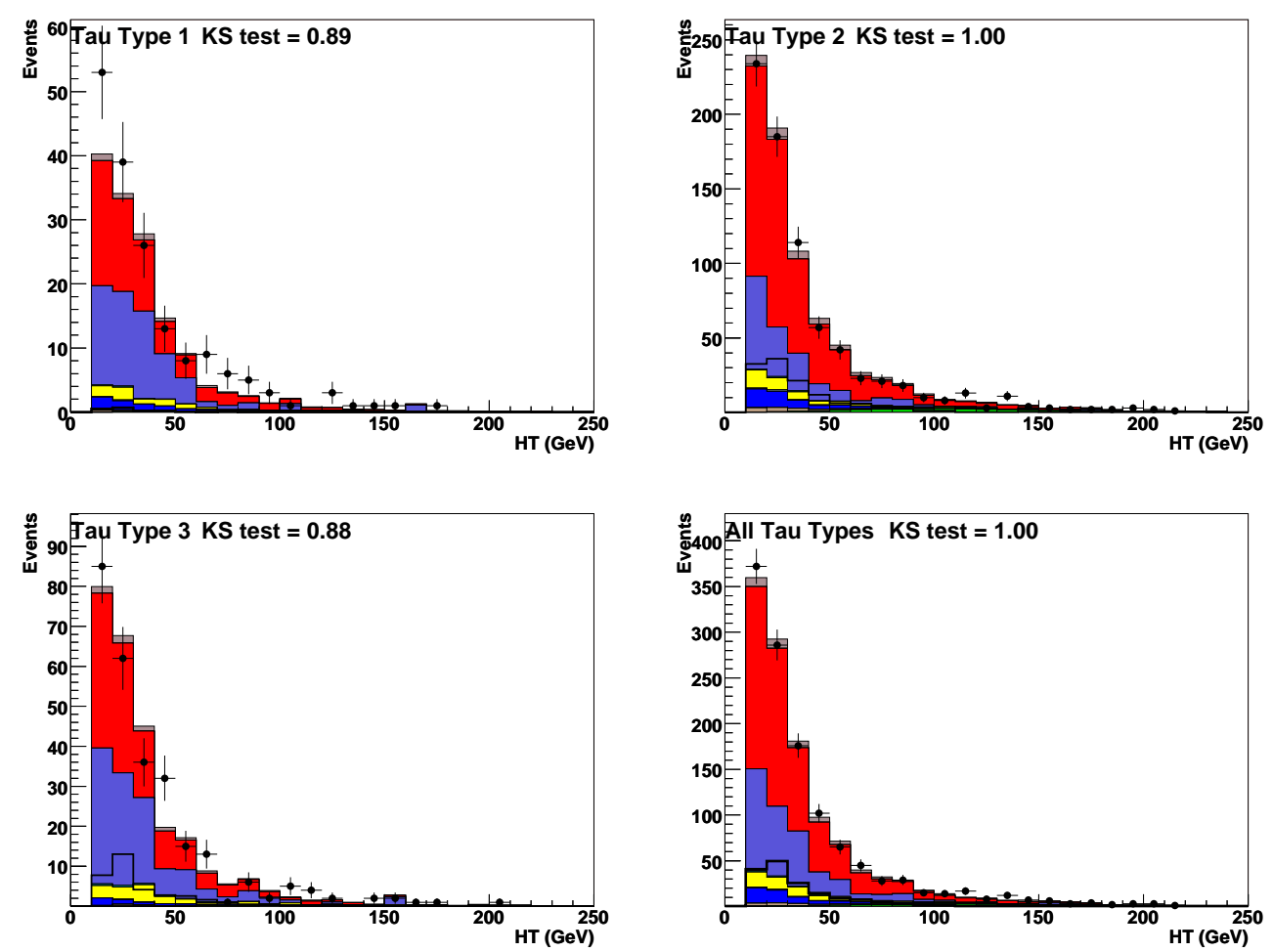

Figure B.6: Sum of trasverse energy of all jets $\left(H_{T}\right)$ before $b$-tagging for: Type 1 taus (upper left), Type 2 (upper right), Type 3 (lower left), and all types (lower right). 

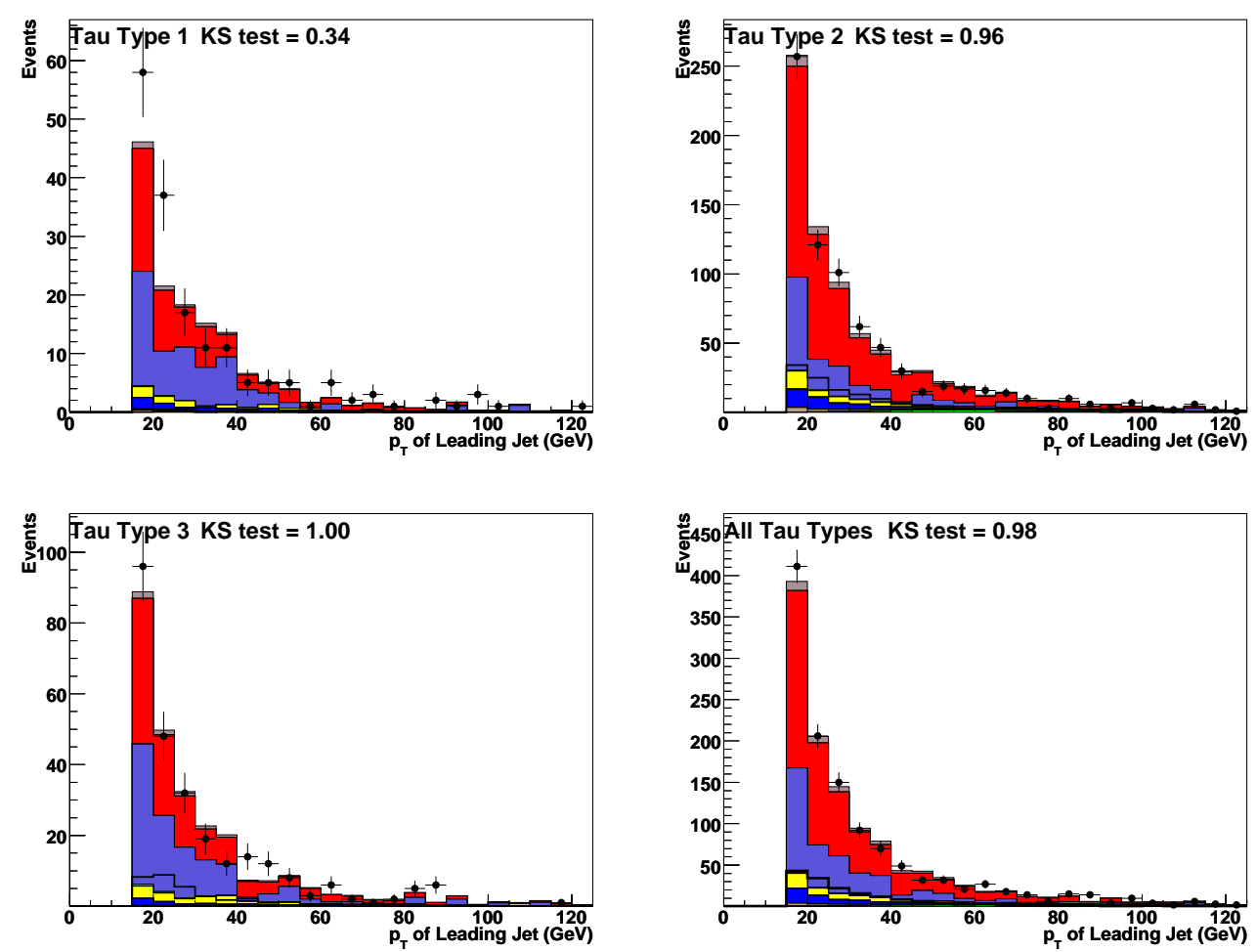

Figure B.7: Leading (highest- $E_{T}$ ) jet $p_{T}$ before $b$-tagging for: Type 1 taus (upper left), Type 2 (upper right), Type 3 (lower left), and all types (lower right). 

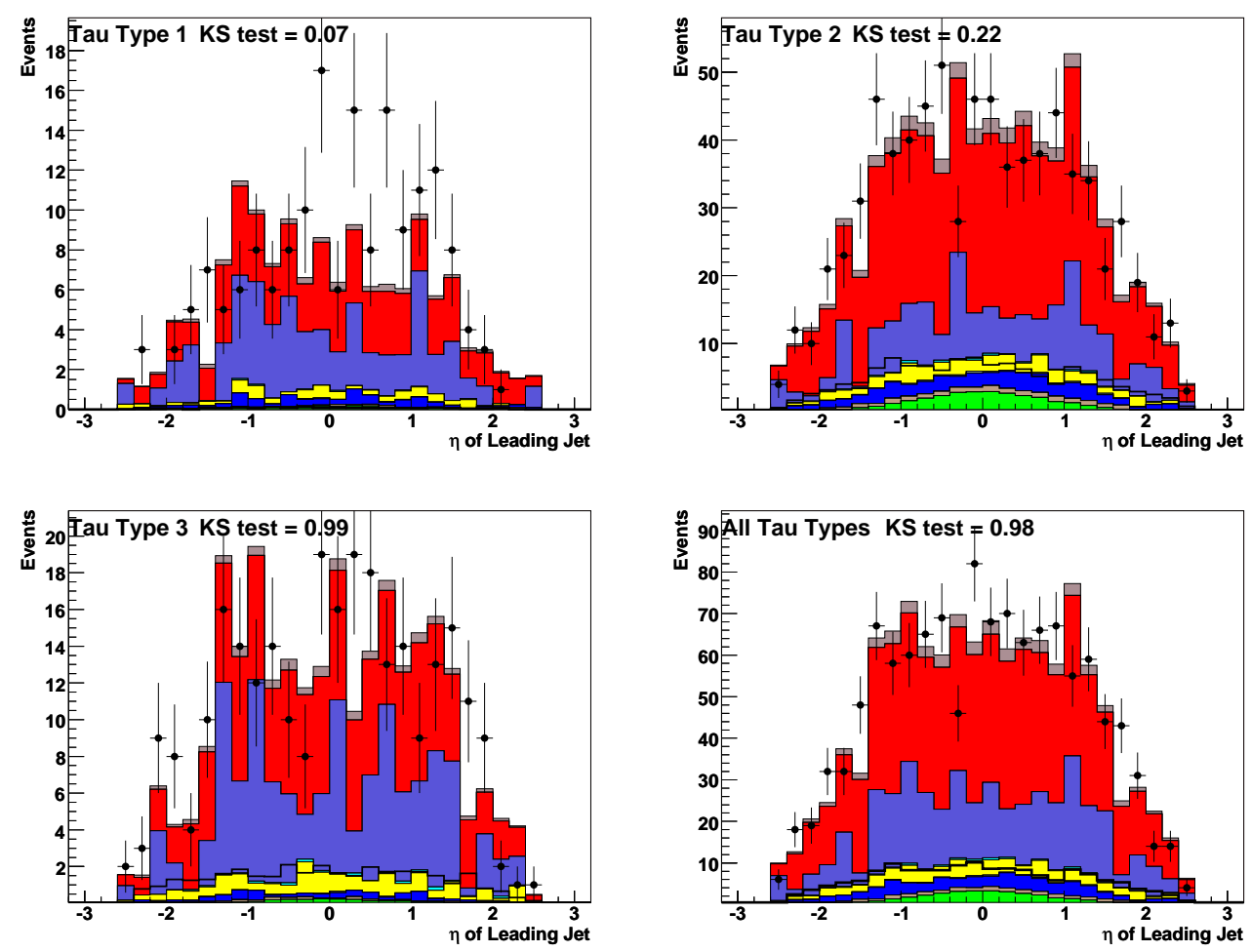

Figure B.8: Leading (highest- $E_{T}$ ) jet $\eta$ before $b$-tagging for: Type 1 taus (upper left), Type 2 (upper right), Type 3 (lower left), and all types (lower right). 

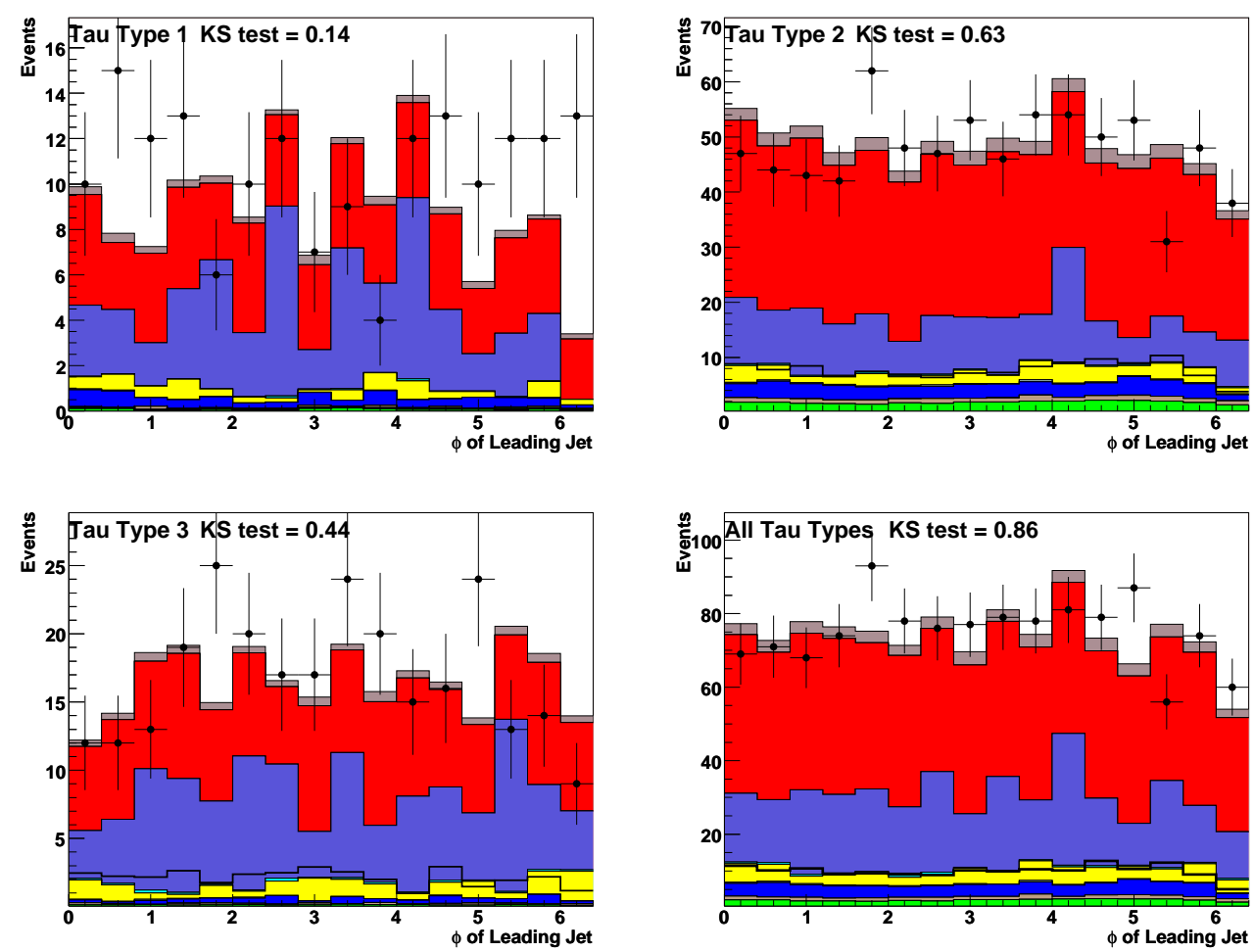

Figure B.9: Leading (highest- $E_{T}$ ) jet $\phi$ before $b$-tagging for: Type 1 taus (upper left), Type 2 (upper right), Type 3 (lower left), and all types (lower right). 

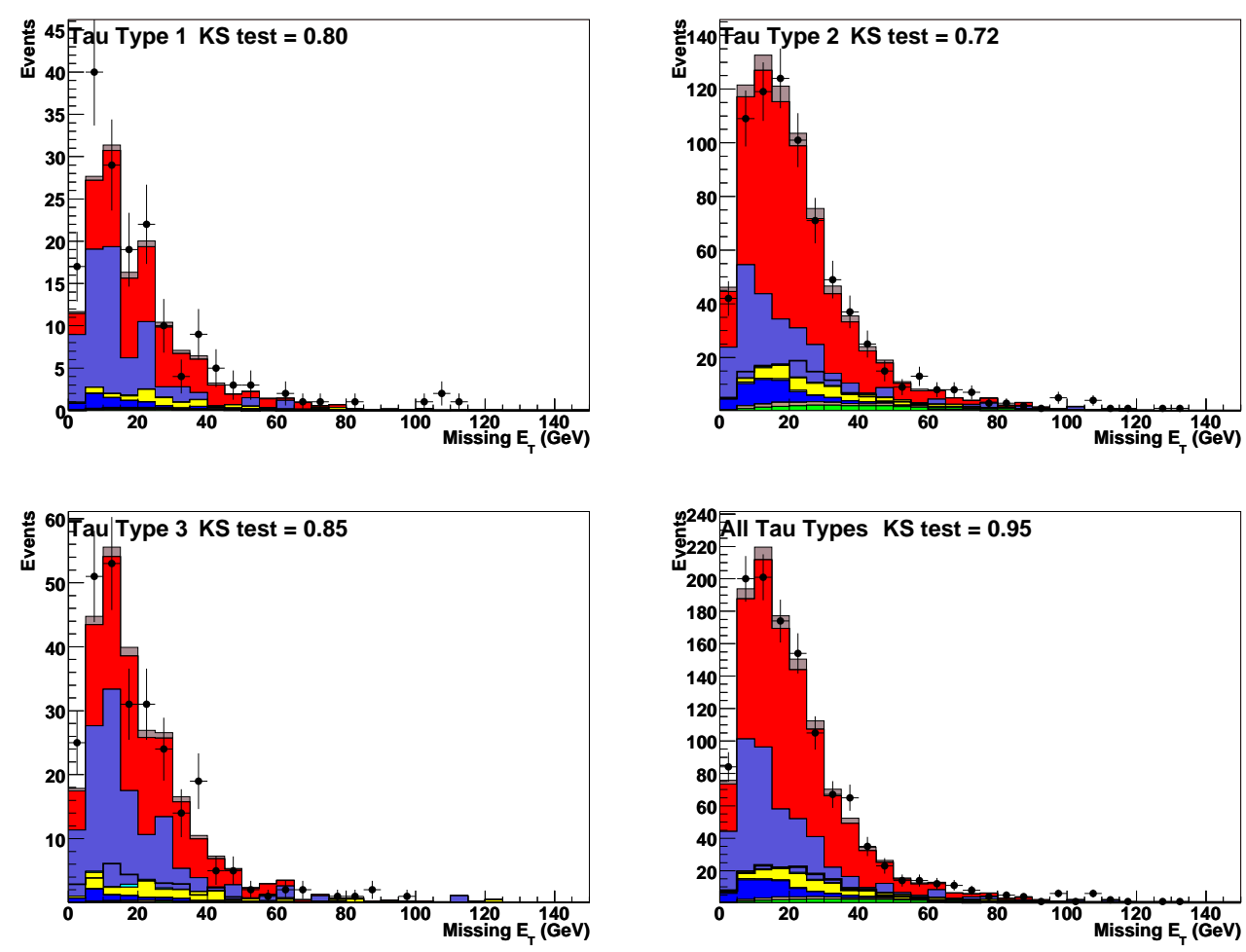

Figure B.10: Missing transverse energy $\left(\mathbb{E}_{T}\right)$ before $b$-tagging for: Type 1 taus (upper left), Type 2 (upper right), Type 3 (lower left), and all types (lower right). 

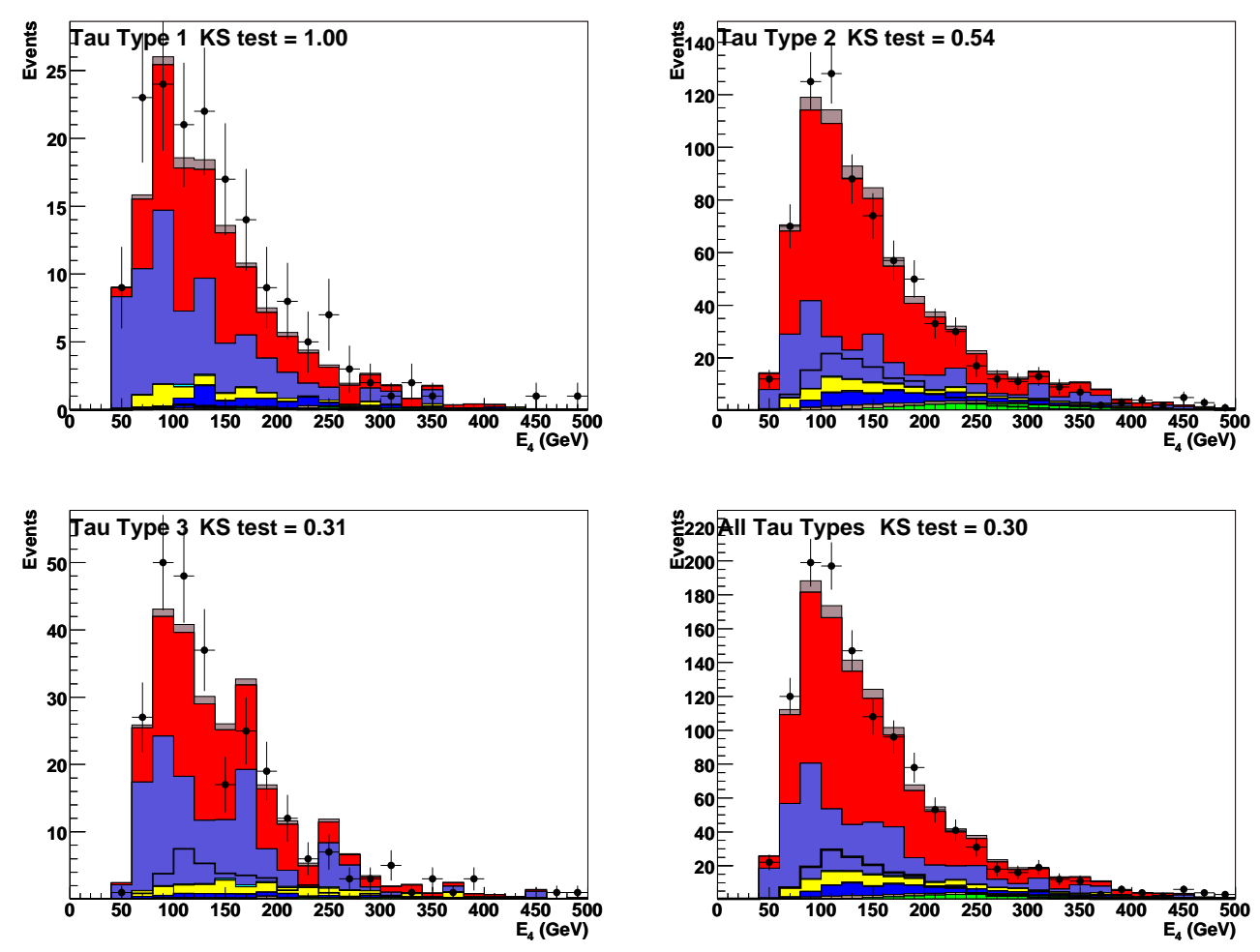

Figure B.11: Energy from 4-vector sum of muon, tau, and $\mathbb{E}_{T}\left(E_{4}\right)$ before $b$ tagging for: Type 1 taus (upper left), Type 2 (upper right), Type 3 (lower left), and all types (lower right). 

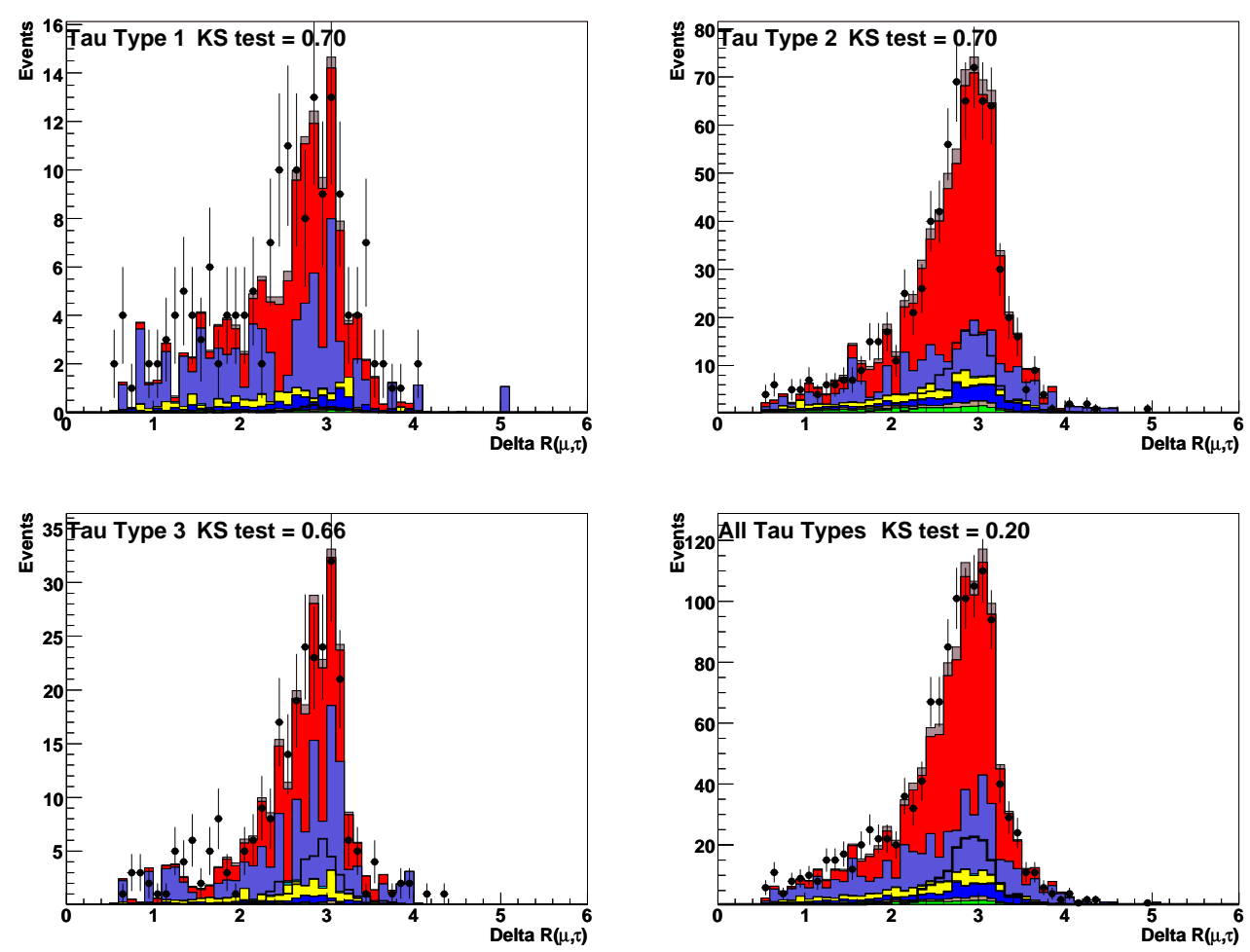

Figure B.12: $\Delta R(\mu, \tau)$, where $\Delta R=\sqrt{(\Delta \eta)^{2}+(\Delta \phi)^{2}}$, before $b$-tagging for: Type 1 taus (upper left), Type 2 (upper right), Type 3 (lower left), and all types (lower right). 

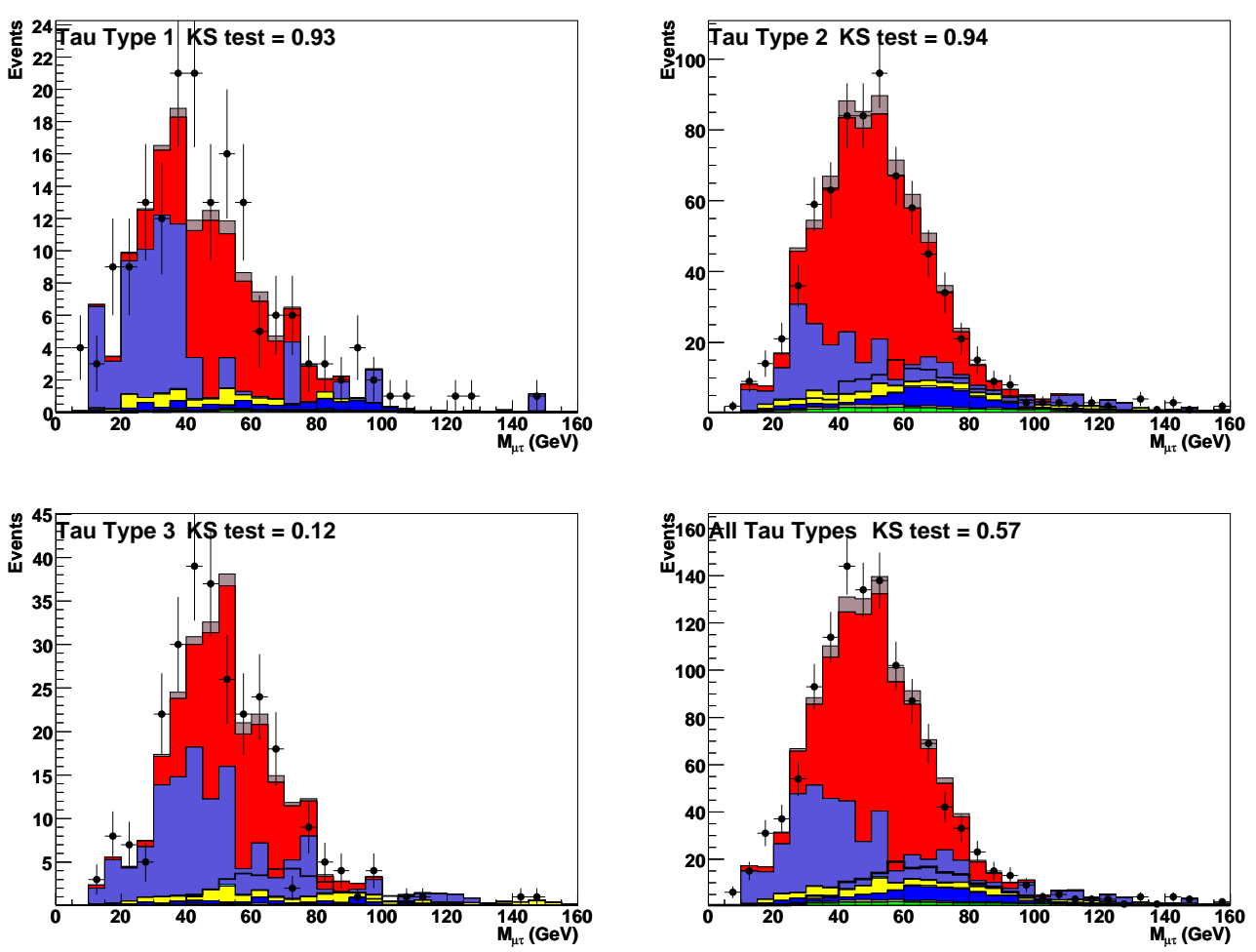

Figure B.13: The $\mu, \tau$ invariant mass before $b$-tagging for: Type 1 taus (upper left), Type 2 (upper right), Type 3 (lower left), and all types (lower right). 

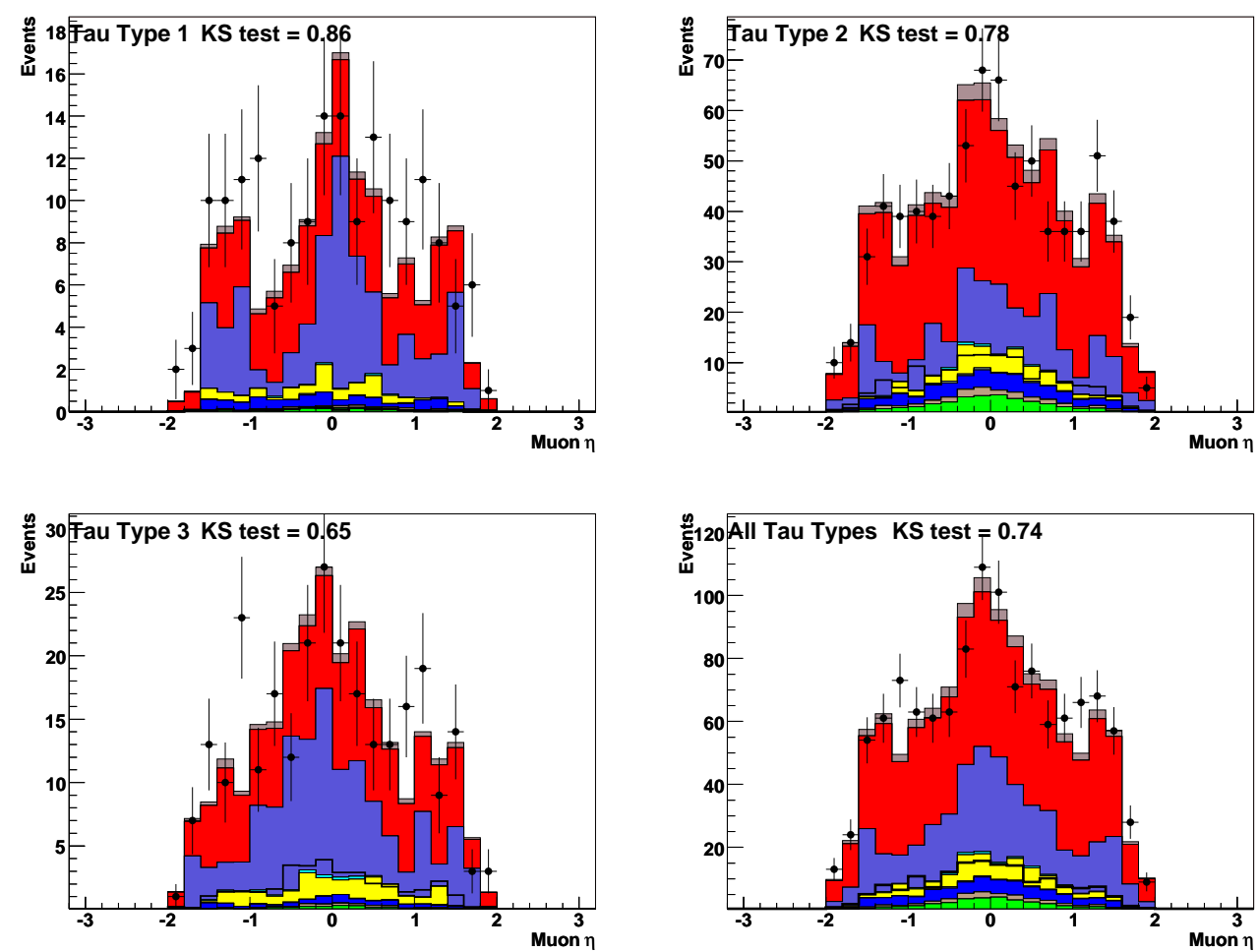

Figure B.14: Muon $\eta$ before $b$-tagging for: Type 1 taus (upper left), Type 2 (upper right), Type 3 (lower left), and all types (lower right). 

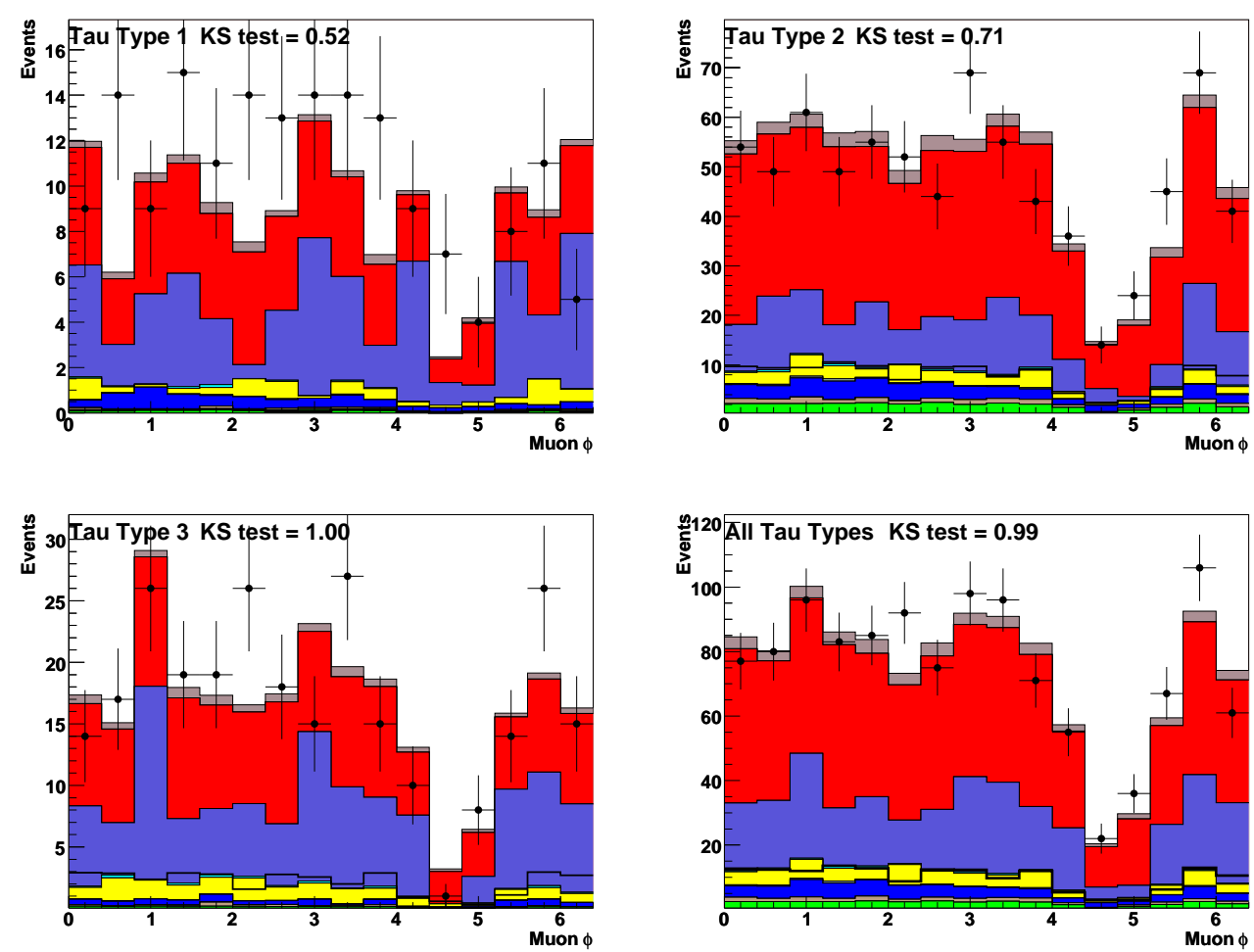

Figure B.15: Muon $\phi$ before $b$-tagging for: Type 1 taus (upper left), Type 2 (upper right), Type 3 (lower left), and all types (lower right). The pronounced dip around $\phi=5$ is due to the lack of full instrumentation at the bottom of the detector. 

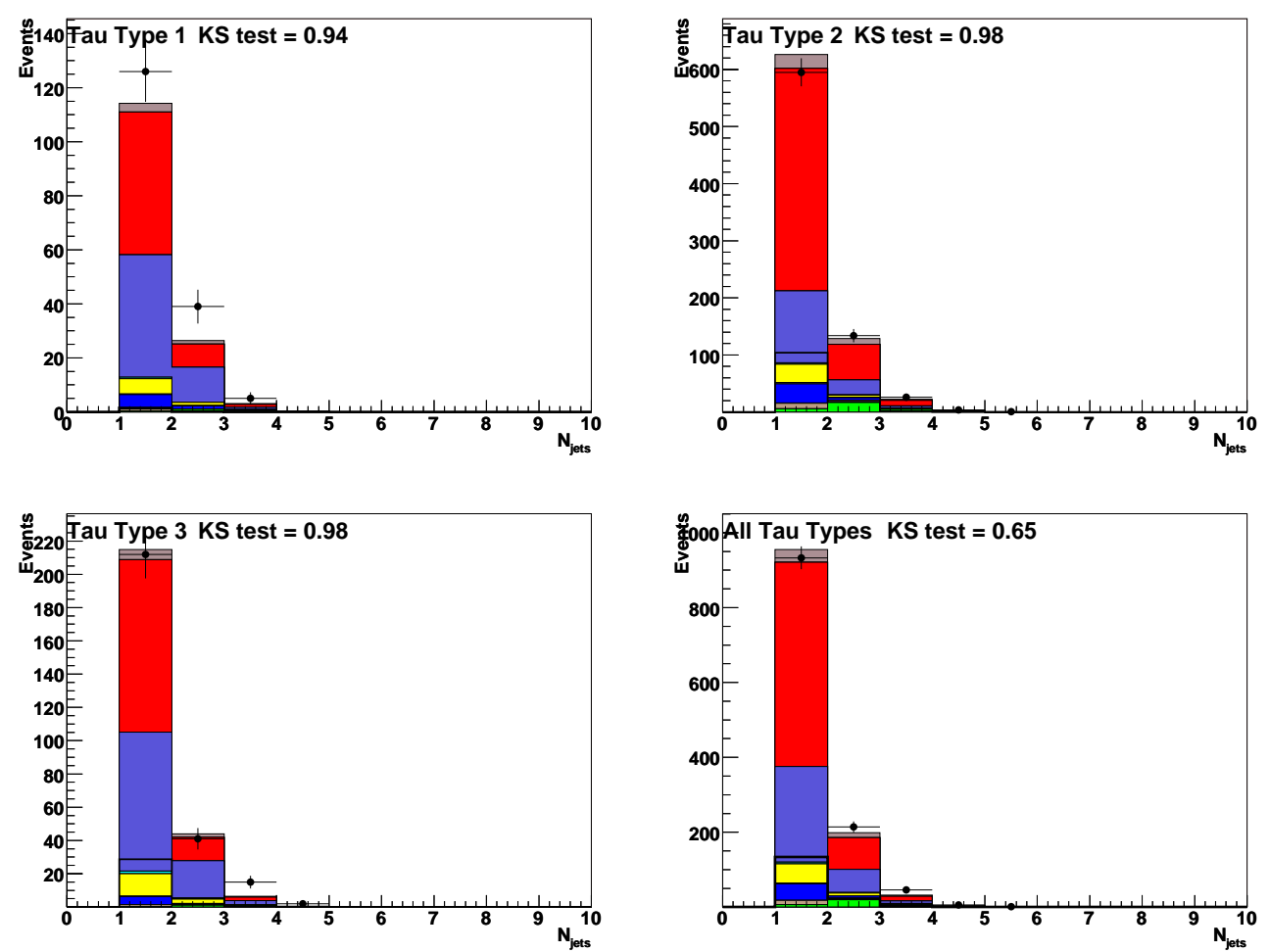

Figure B.16: Jet multiplicity before $b$-tagging for: Type 1 taus (upper left), Type 2 (upper right), Type 3 (lower left), and all types (lower right). 

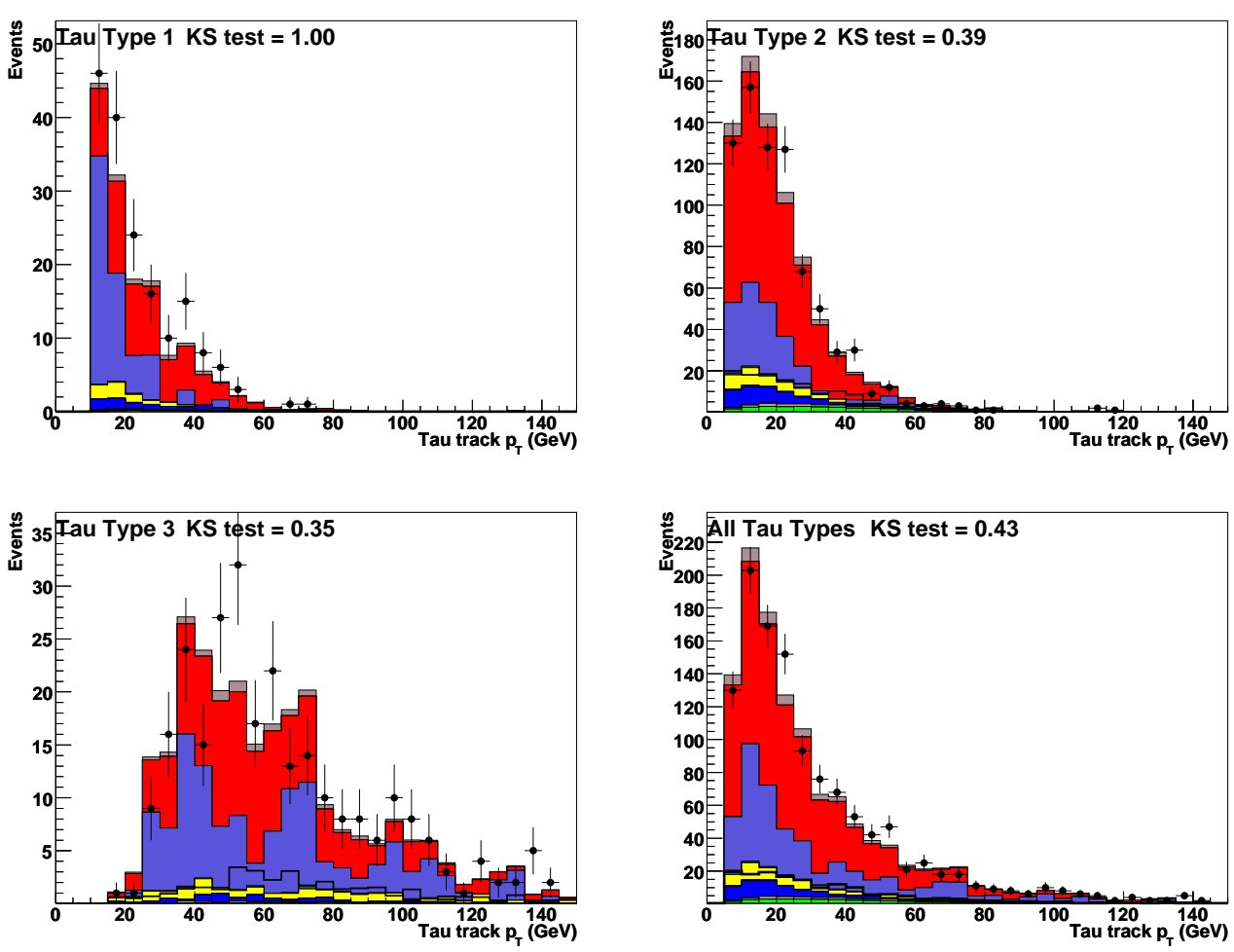

Figure B.17: Tau associated track $p_{T}$ before $b$-tagging for: Type 1 taus (upper left), Type 2 (upper right), Type 3 (lower left), and all types (lower right). In tau type 3 , it is the $p_{T}$ sum of all tracks. 

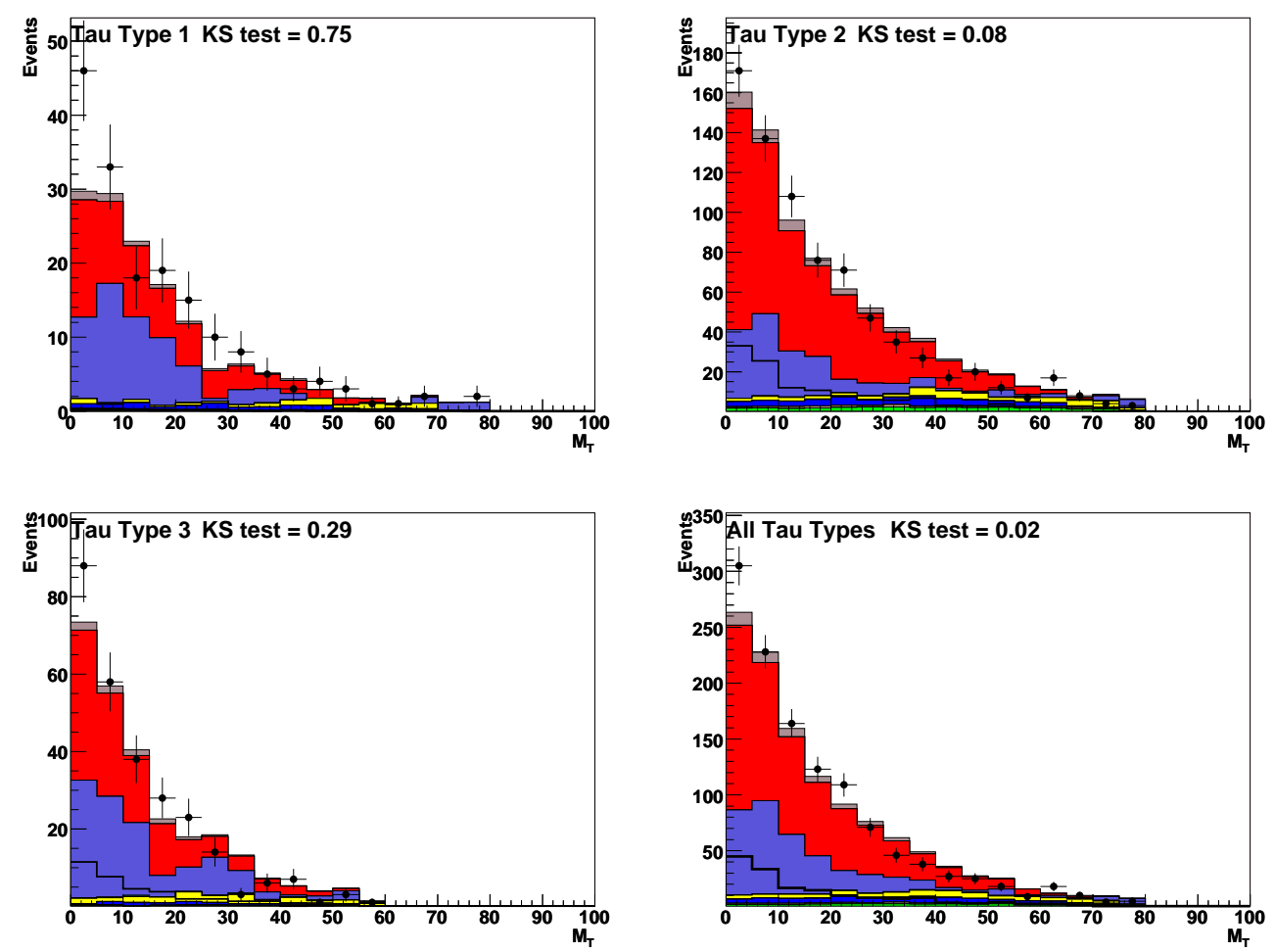

Figure B.18: The transverse mass $\left(M_{T}=\sqrt{\left(2 E_{T} E_{T \mu}\left(1-\cos \left(\Delta \phi\left(\mu, \mathbb{E}_{T}\right)\right)\right)\right.}\right)$ before $b$-tagging for: Type 1 taus (upper left), Type 2 (upper right), Type 3 (lower left), and all types (lower right). 

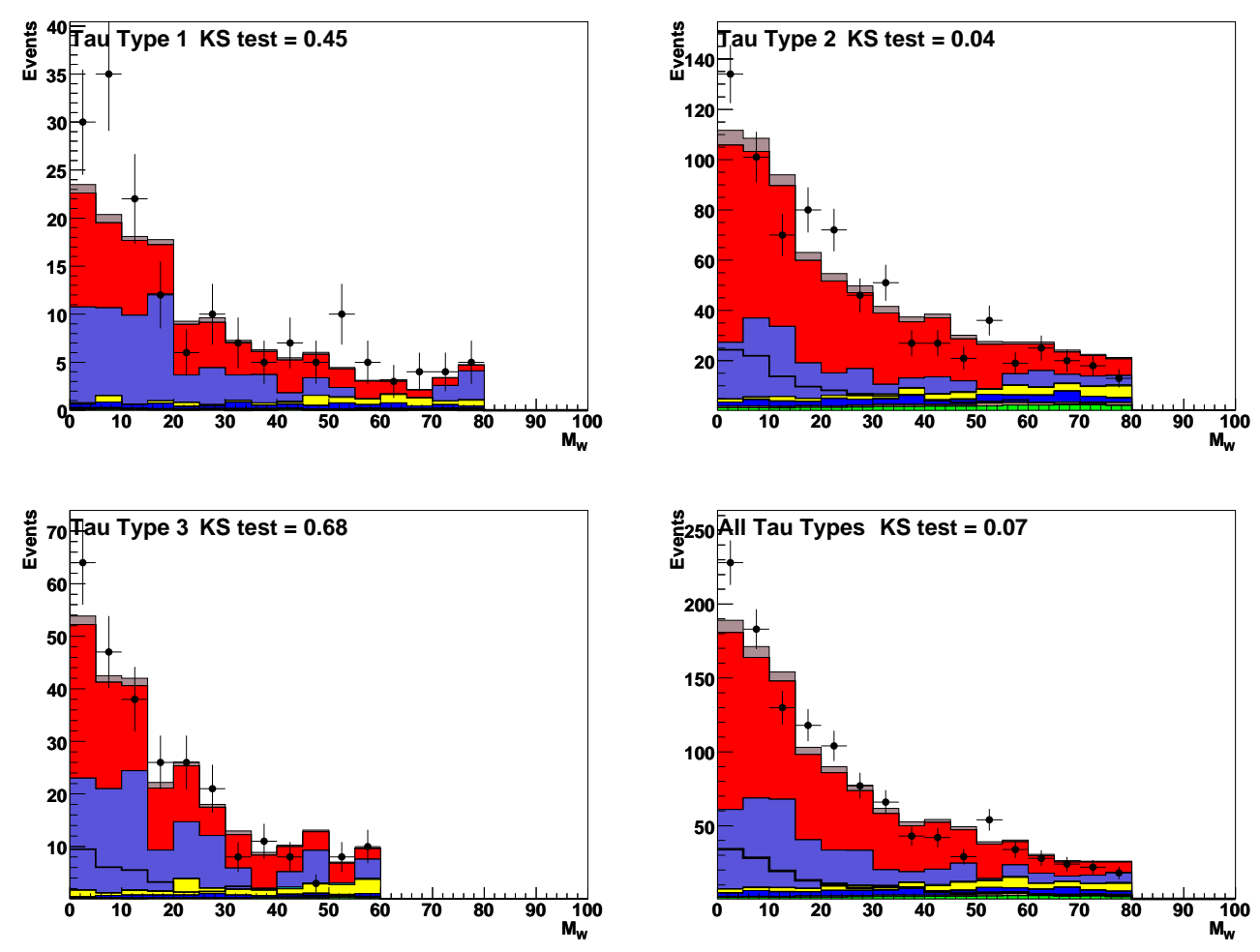

Figure B.19: The $W$ mass variable $\left(M_{W}=\sqrt{\left(2 E_{T} E_{\mu}^{2} / p_{T \mu}\left(1-\cos \left(\Delta \phi\left(\mu, E_{T}\right)\right)\right)\right)}\right)$ before $b$-tagging for: Type 1 taus (upper left), Type 2 (upper right), Type 3 (lower left), and all types (lower right). 

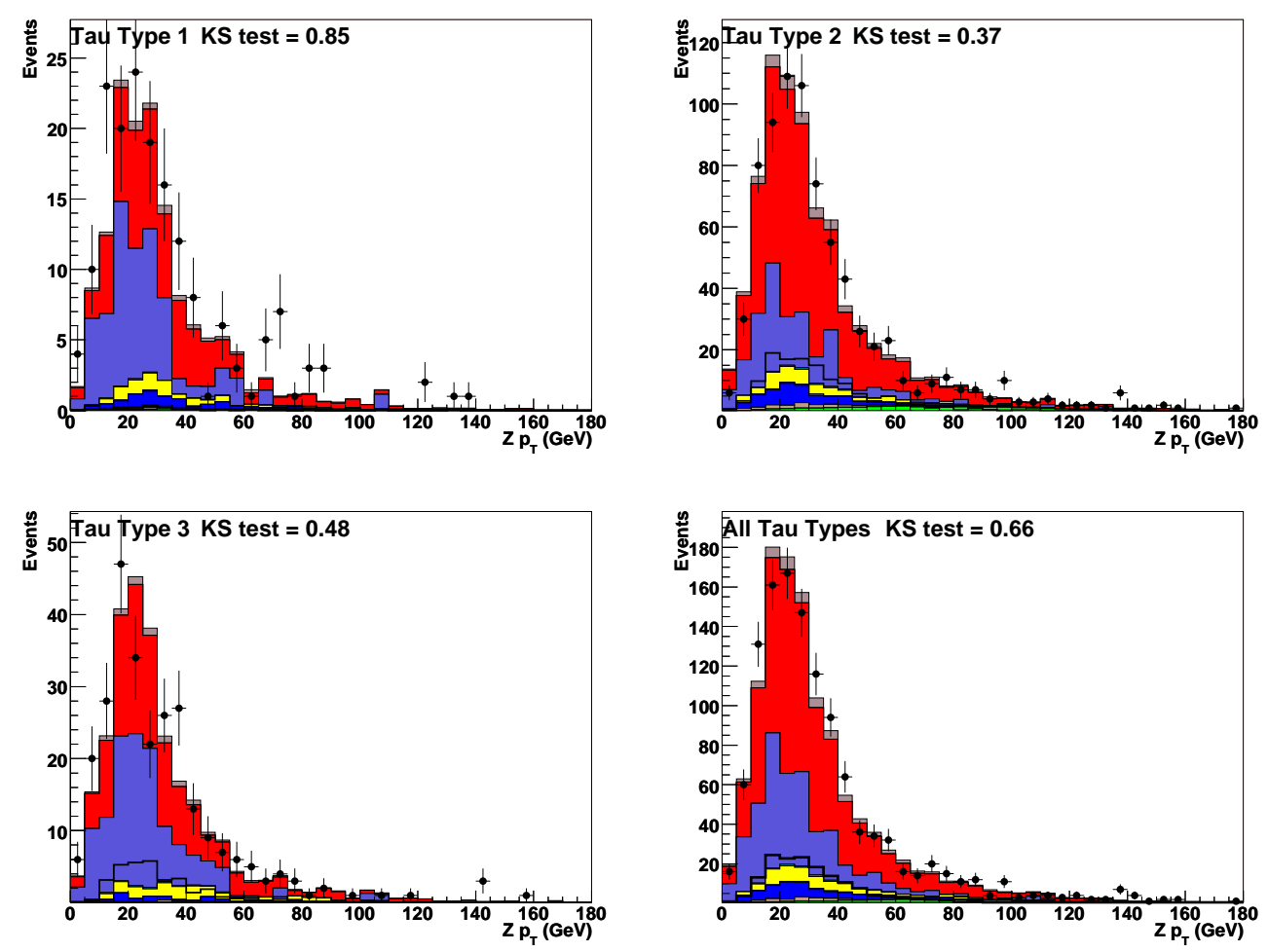

Figure B.20: The $Z p_{T}$ variable, $Z p_{T}=\left|\boldsymbol{p}_{\boldsymbol{T}}^{\boldsymbol{\mu}}+\boldsymbol{p}_{\boldsymbol{T}}^{\boldsymbol{\tau}}+\boldsymbol{E}_{\boldsymbol{T}}\right|$, before $b$-tagging for: Type 1 taus (upper left), Type 2 (upper right), Type 3 (lower left), and all types (lower right) using the Single Muon or $\mathrm{Mu}+\mathrm{Tau} \mathrm{OR}$. 


\section{B.2 Data/Monte Carlo Plots After $b$-tagging}
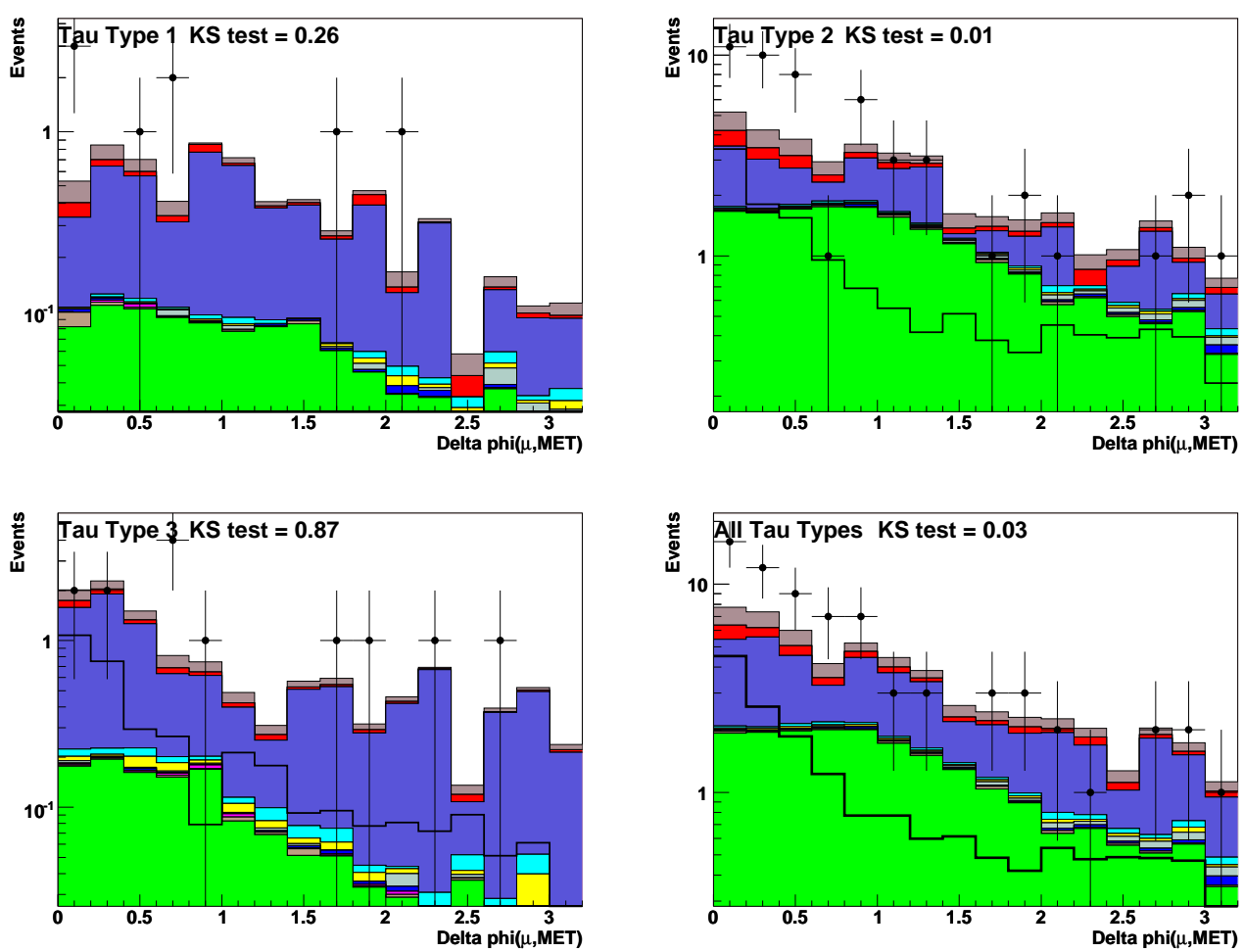

Figure B.21: $\Delta \phi\left(\mu, \mathbb{E}_{T}\right)$ after $b$-tagging for: Type 1 taus (upper left), Type 2 (upper right), Type 3 (lower left), and all types (lower right). 

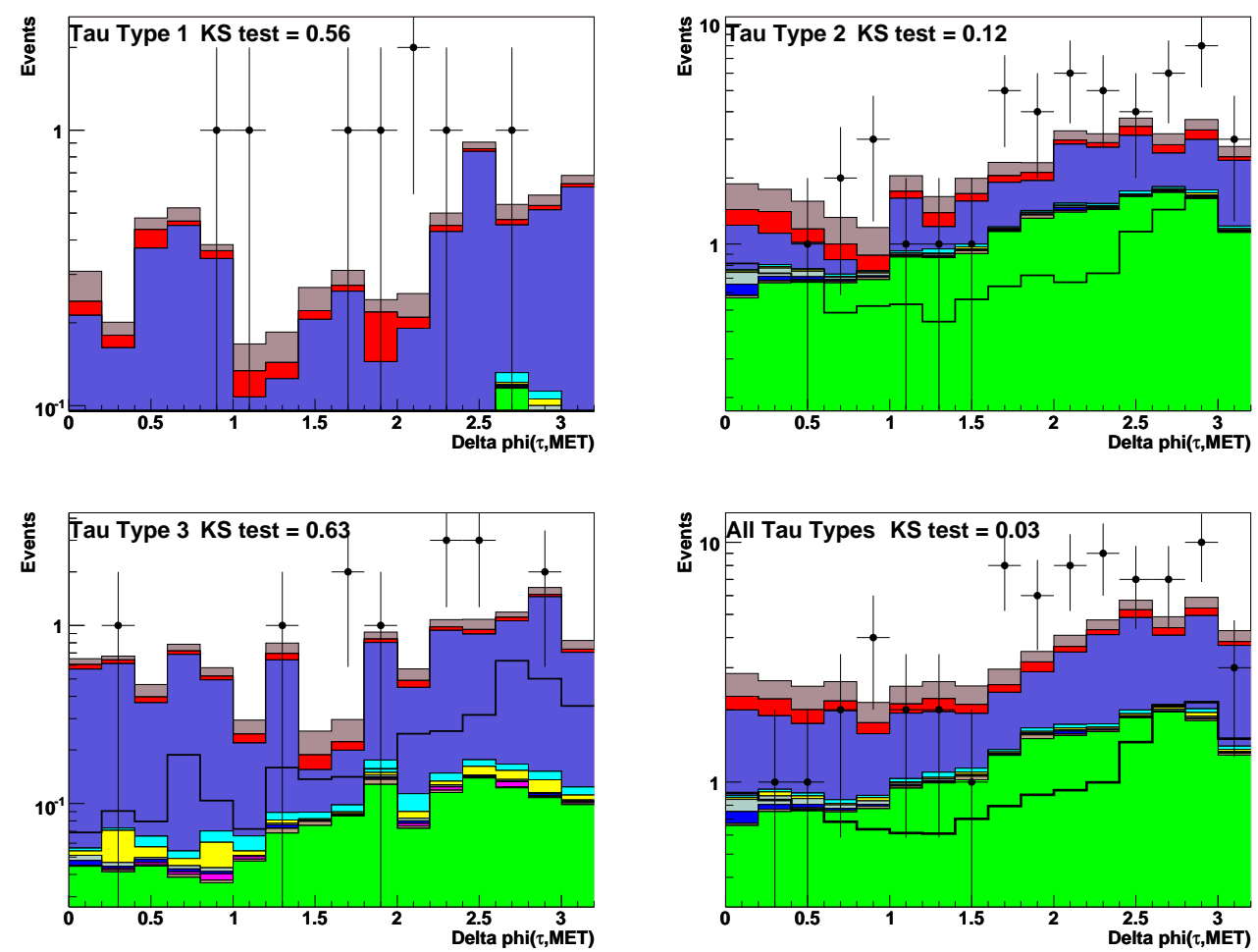

Figure B.22: $\Delta \phi\left(\tau, \mathbb{E}_{T}\right)$ after $b$-tagging for: Type 1 taus (upper left), Type 2 (upper right), Type 3 (lower left), and all types (lower right). 

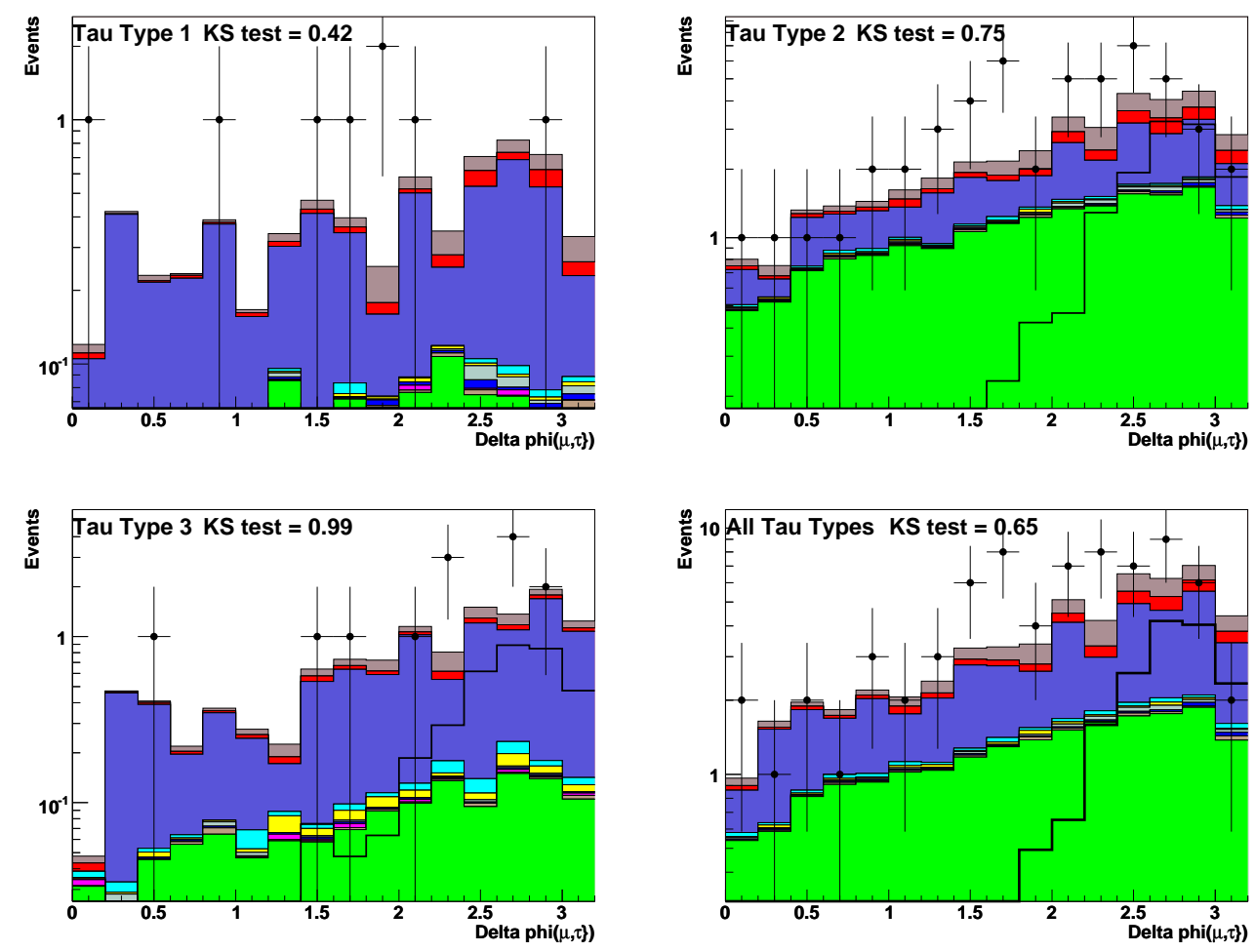

Figure B.23: $\Delta \phi(\mu, \tau)$ after $b$-tagging for: Type 1 taus (upper left), Type 2 (upper right), Type 3 (lower left), and all types (lower right). 

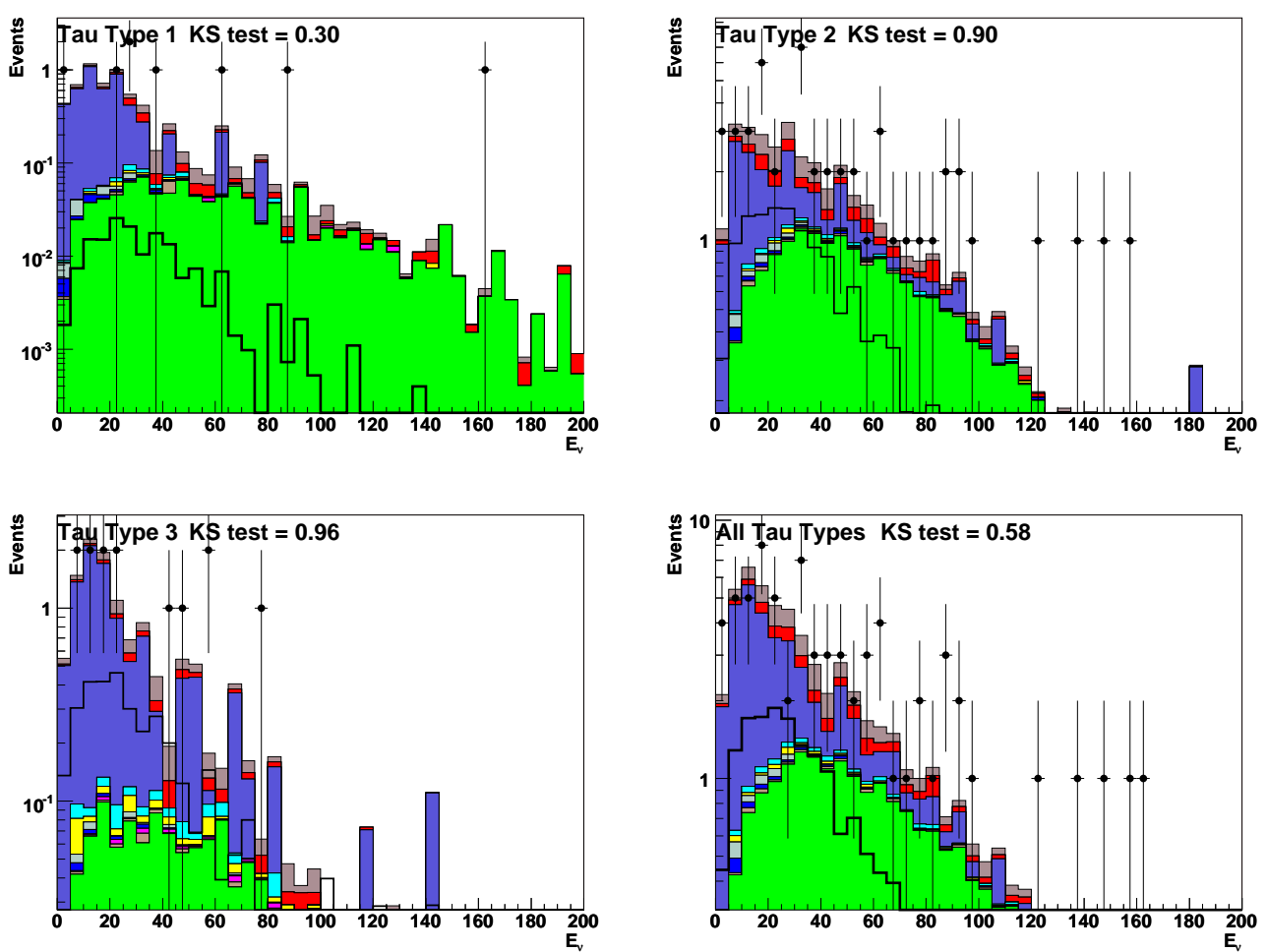

Figure B.24: The Enu (or $E_{\nu}$ ) variable, $E_{\nu} \equiv E_{T} E_{\mu} / p_{T \mu}$ after $b$-tagging for: Type 1 taus (upper left), Type 2 (upper right), Type 3 (lower left), and all types (lower right). 

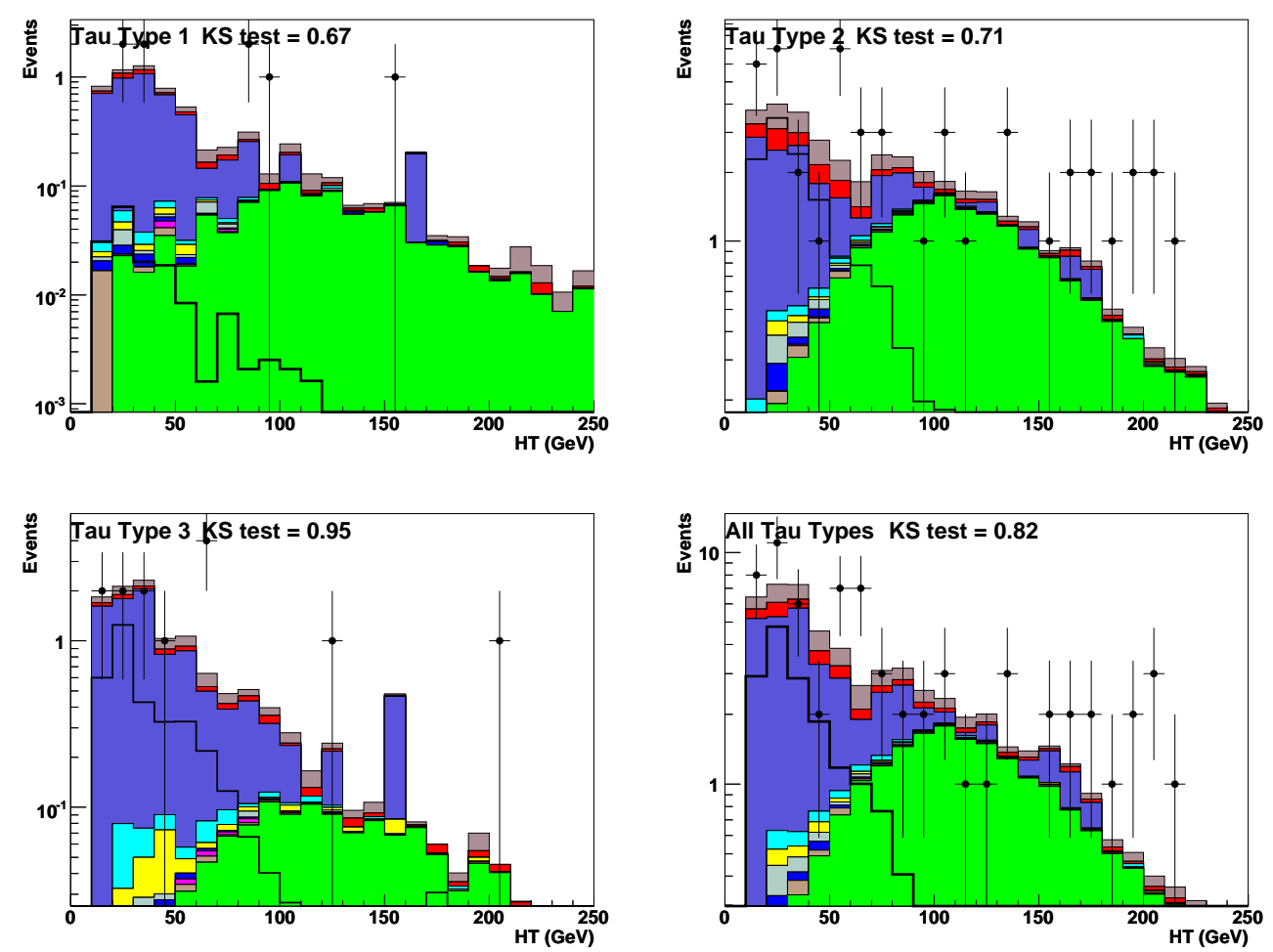

Figure B.25: Sum of trasverse energy of all jets $\left(H_{T}\right)$ after $b$-tagging for: Type 1 taus (upper left), Type 2 (upper right), Type 3 (lower left), and all types (lower right). 

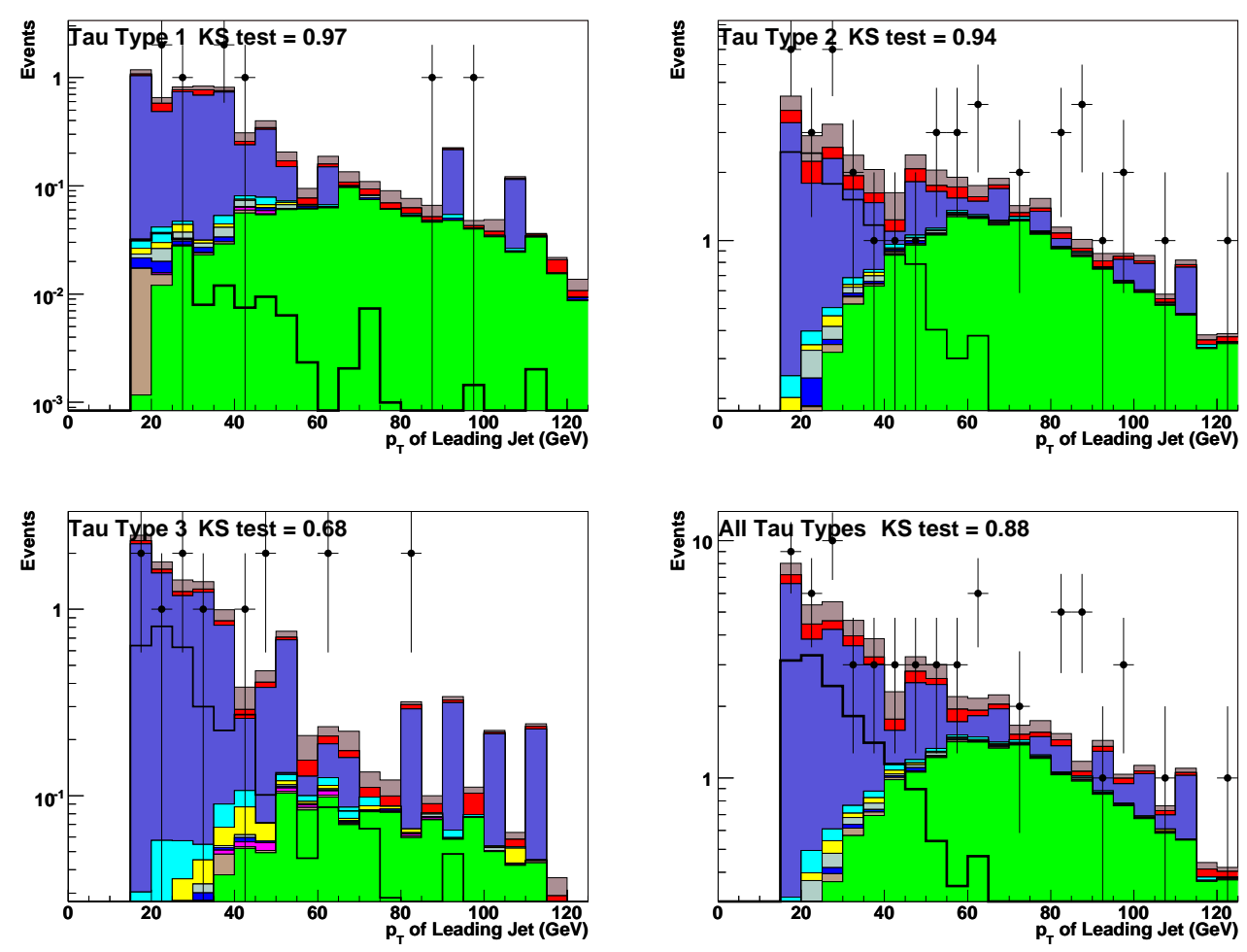

Figure B.26: Leading (highest- $E_{T}$ ) jet $p_{T}$ after $b$-tagging for: Type 1 taus (upper left), Type 2 (upper right), Type 3 (lower left), and all types (lower right). 

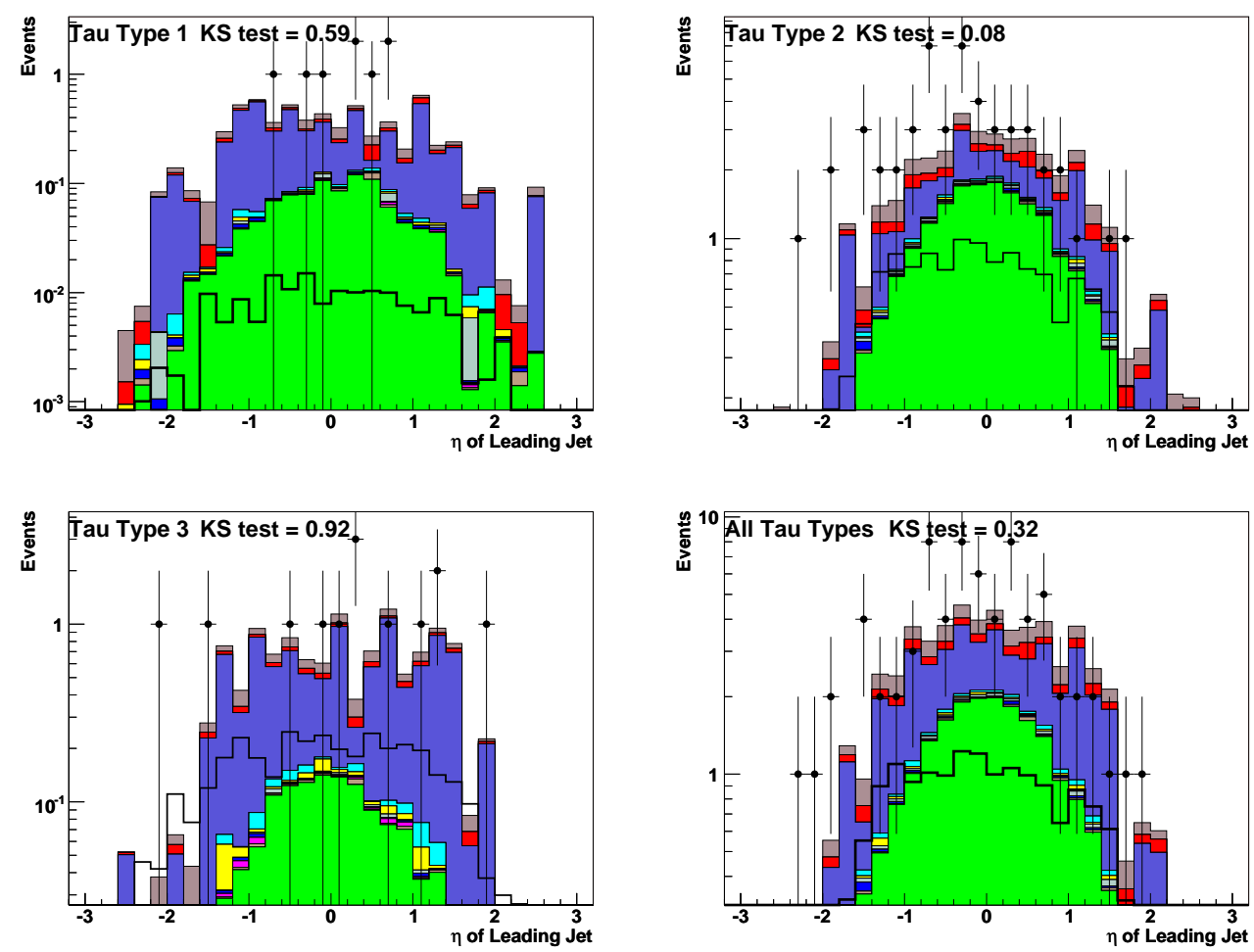

Figure B.27: Leading (highest- $E_{T}$ ) jet $\eta$ after $b$-tagging for: Type 1 taus (upper left), Type 2 (upper right), Type 3 (lower left), and all types (lower right). 

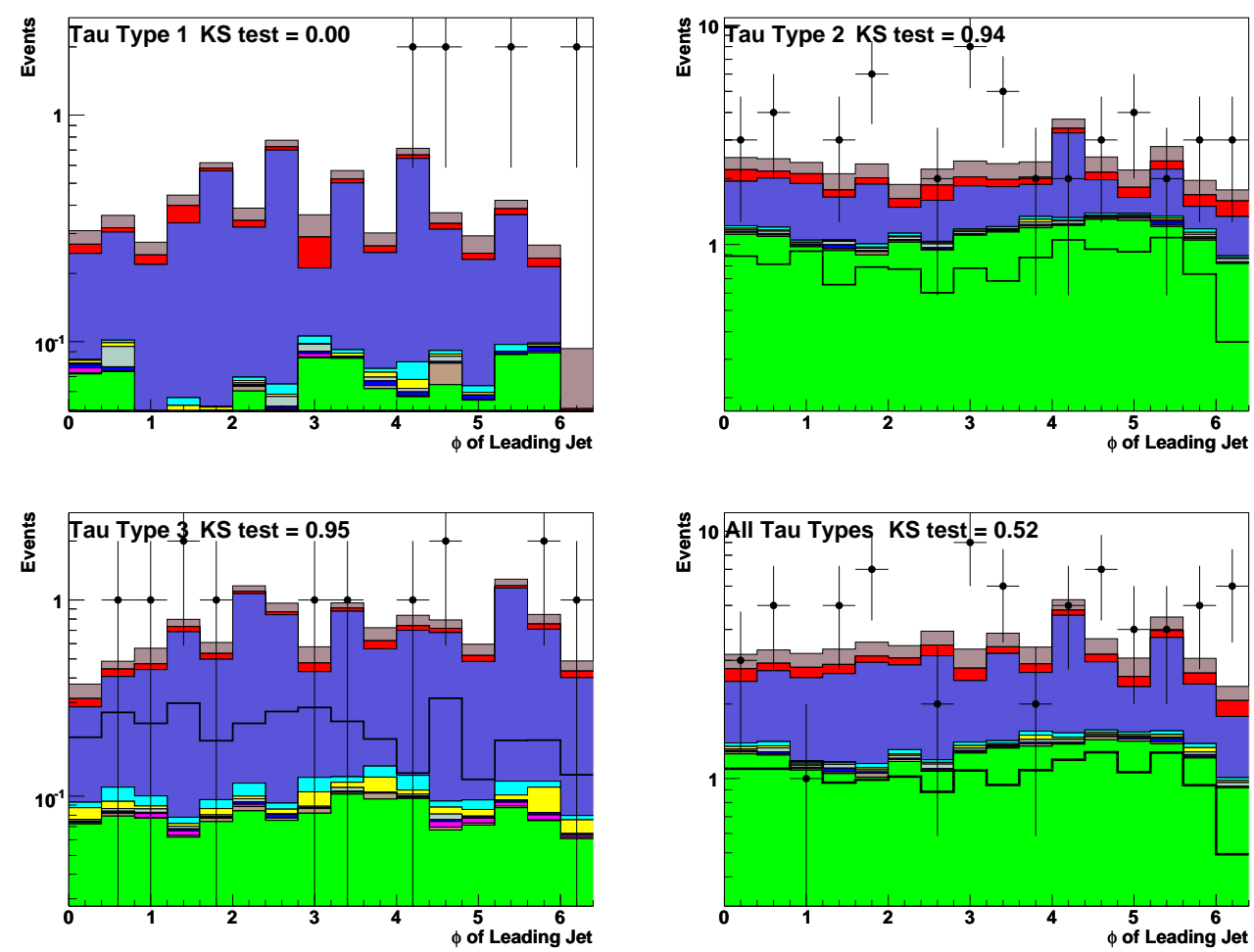

Figure B.28: Leading (highest- $E_{T}$ ) jet $\phi$ after $b$-tagging for: Type 1 taus (upper left), Type 2 (upper right), Type 3 (lower left), and all types (lower right). 

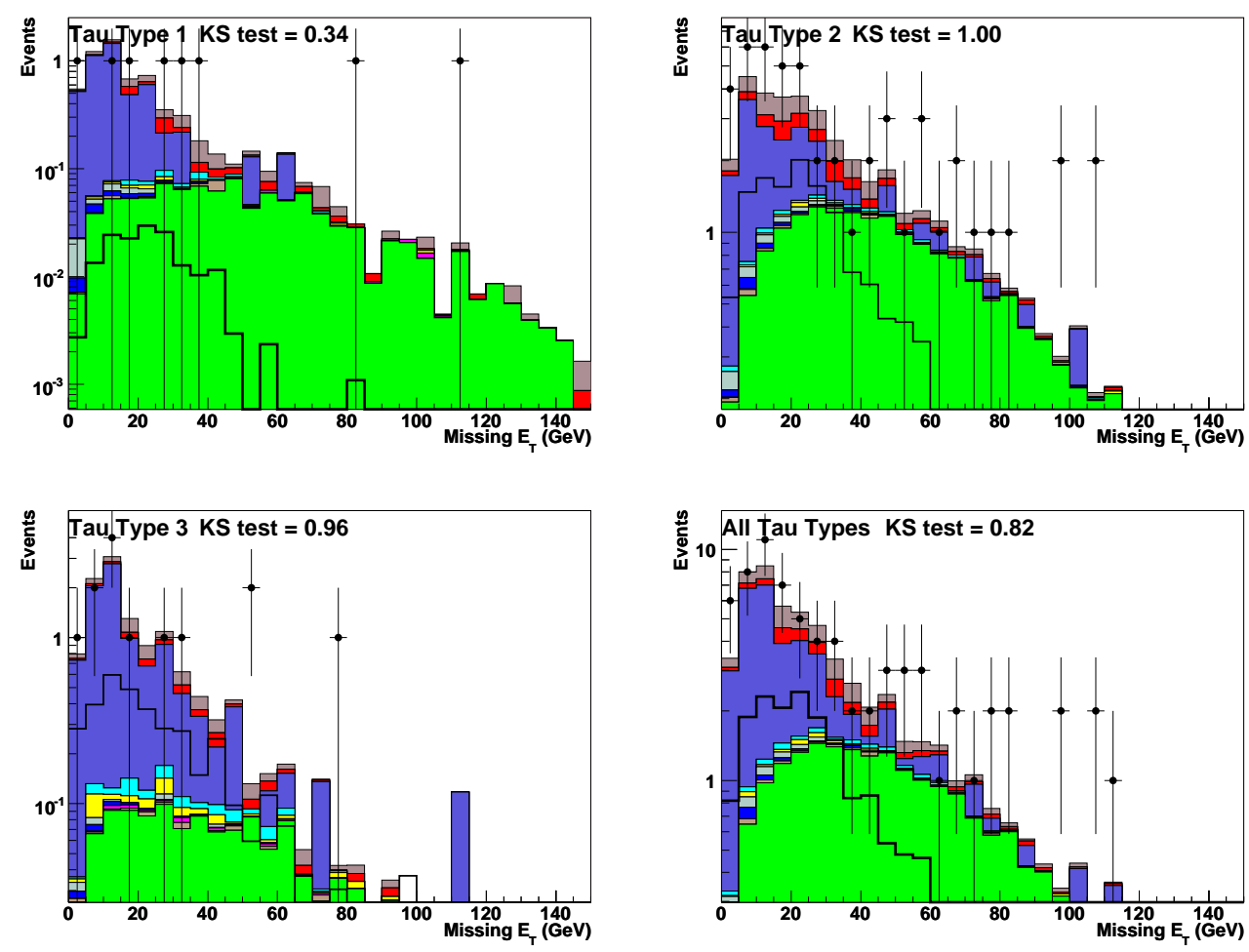

Figure B.29: Missing transverse energy $\left(\mathbb{E}_{T}\right)$ after $b$-tagging for: Type 1 taus (upper left), Type 2 (upper right), Type 3 (lower left), and all types (lower right). 

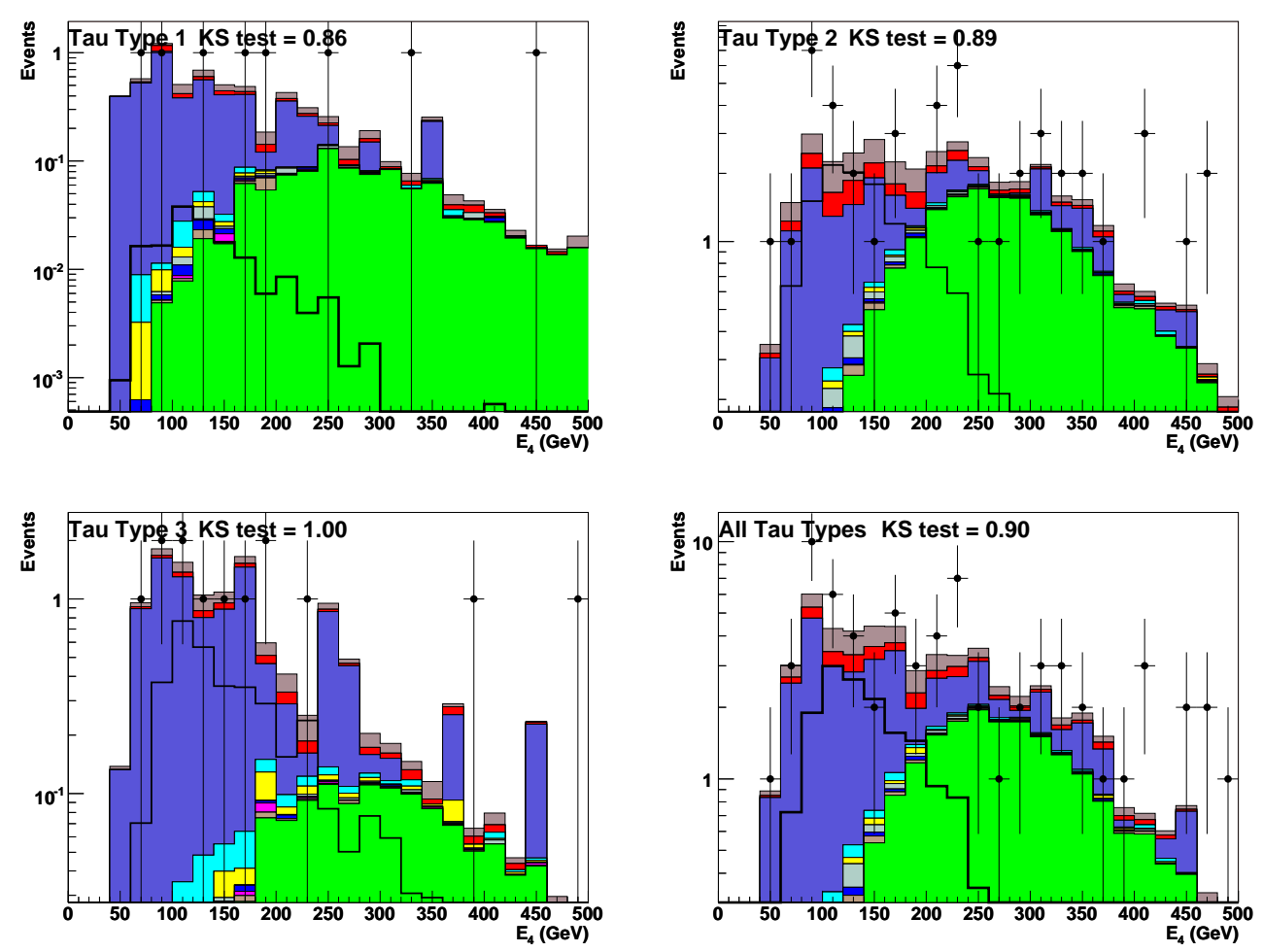

Figure B.30: Energy from 4-vector sum of muon, tau, and $\mathbb{E}_{T}\left(E_{4}\right)$ after $b$ tagging for: Type 1 taus (upper left), Type 2 (upper right), Type 3 (lower left), and all types (lower right). 

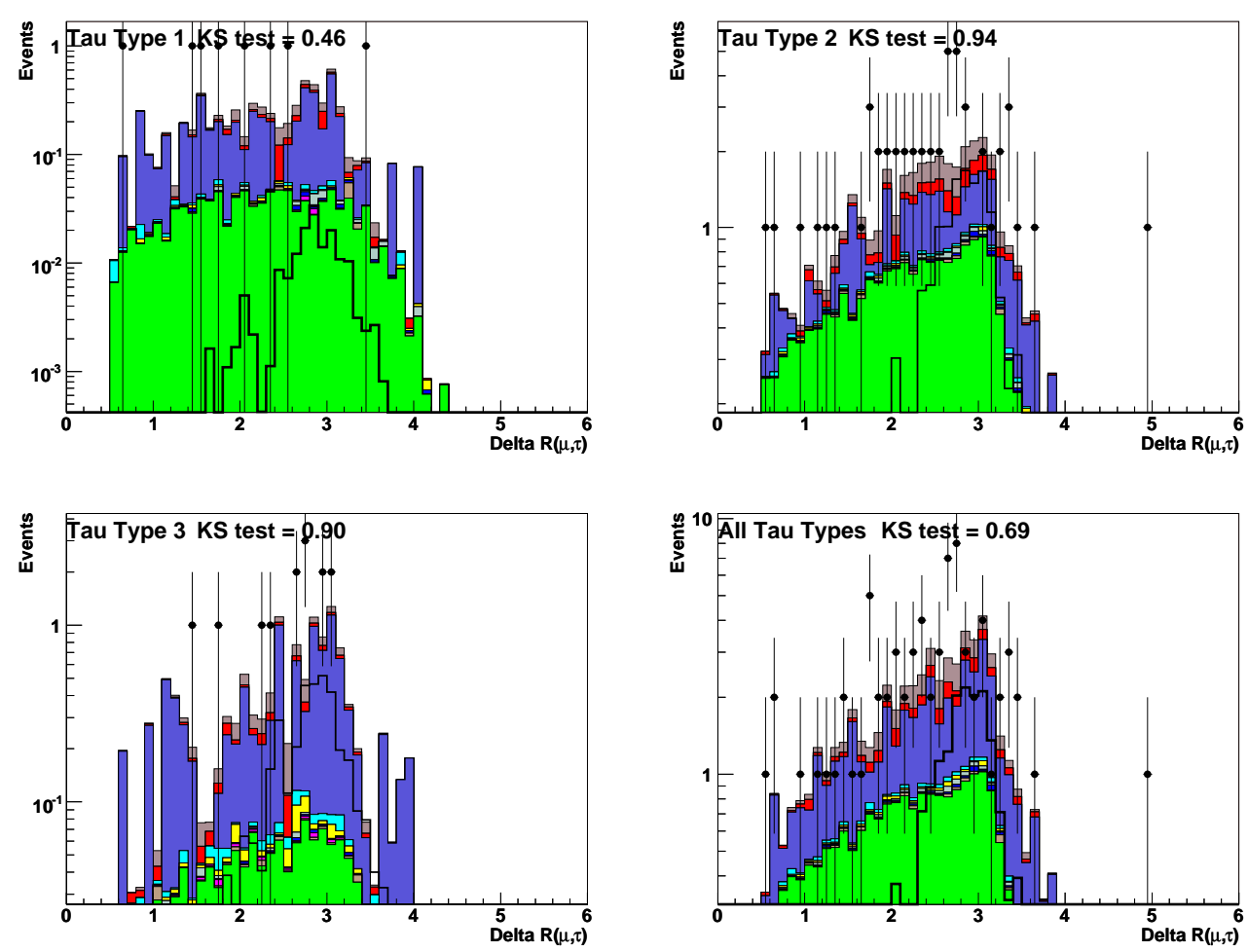

Figure B.31: $\Delta R(\mu, \tau)$, where $\Delta R=\sqrt{(\Delta \eta)^{2}+(\Delta \phi)^{2}}$, after b-tagging for: Type 1 taus (upper left), Type 2 (upper right), Type 3 (lower left), and all types (lower right). 

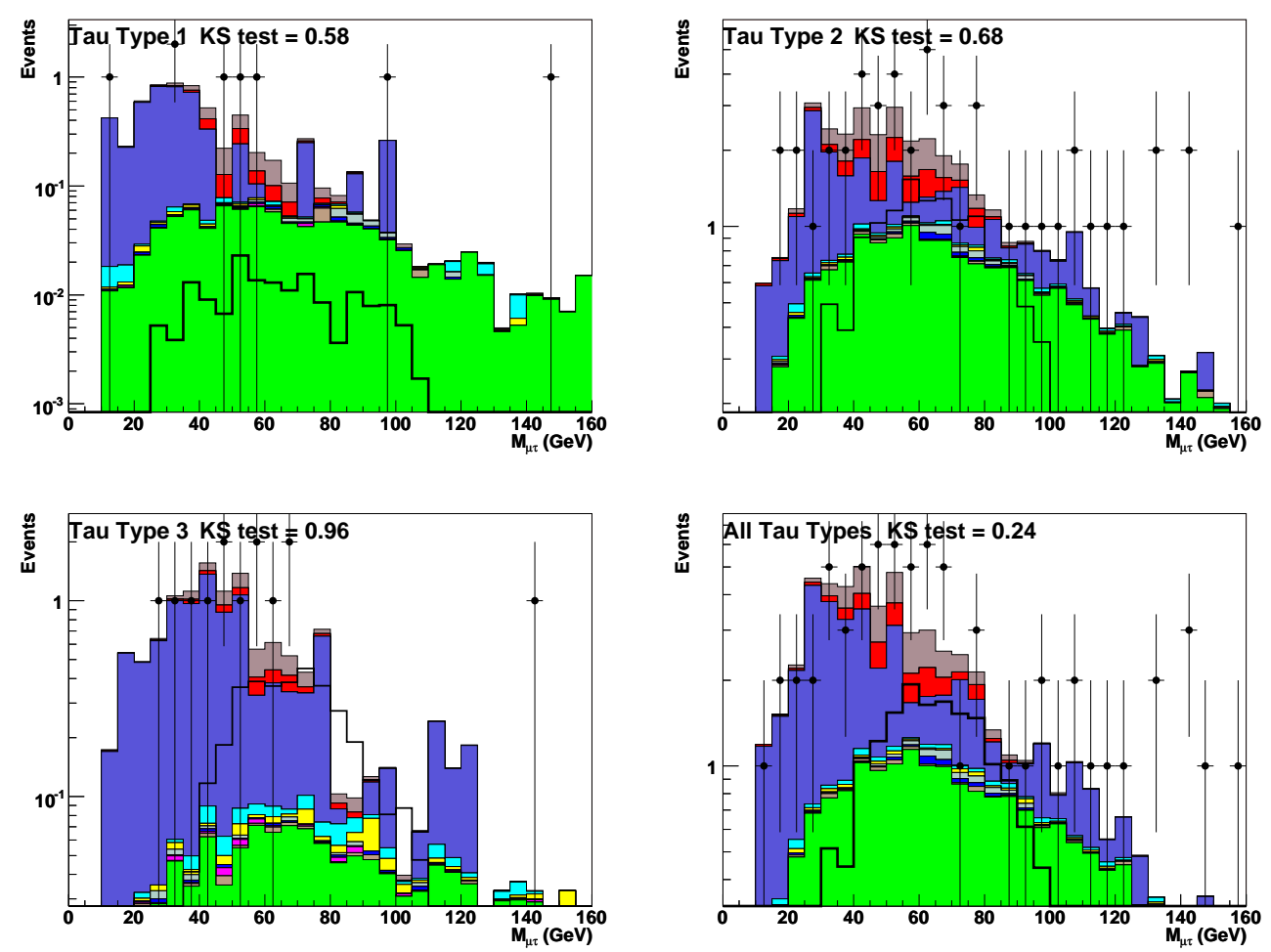

Figure B.32: The $\mu, \tau$ invariant mass after $b$-tagging for: Type 1 taus (upper left), Type 2 (upper right), Type 3 (lower left), and all types (lower right). 

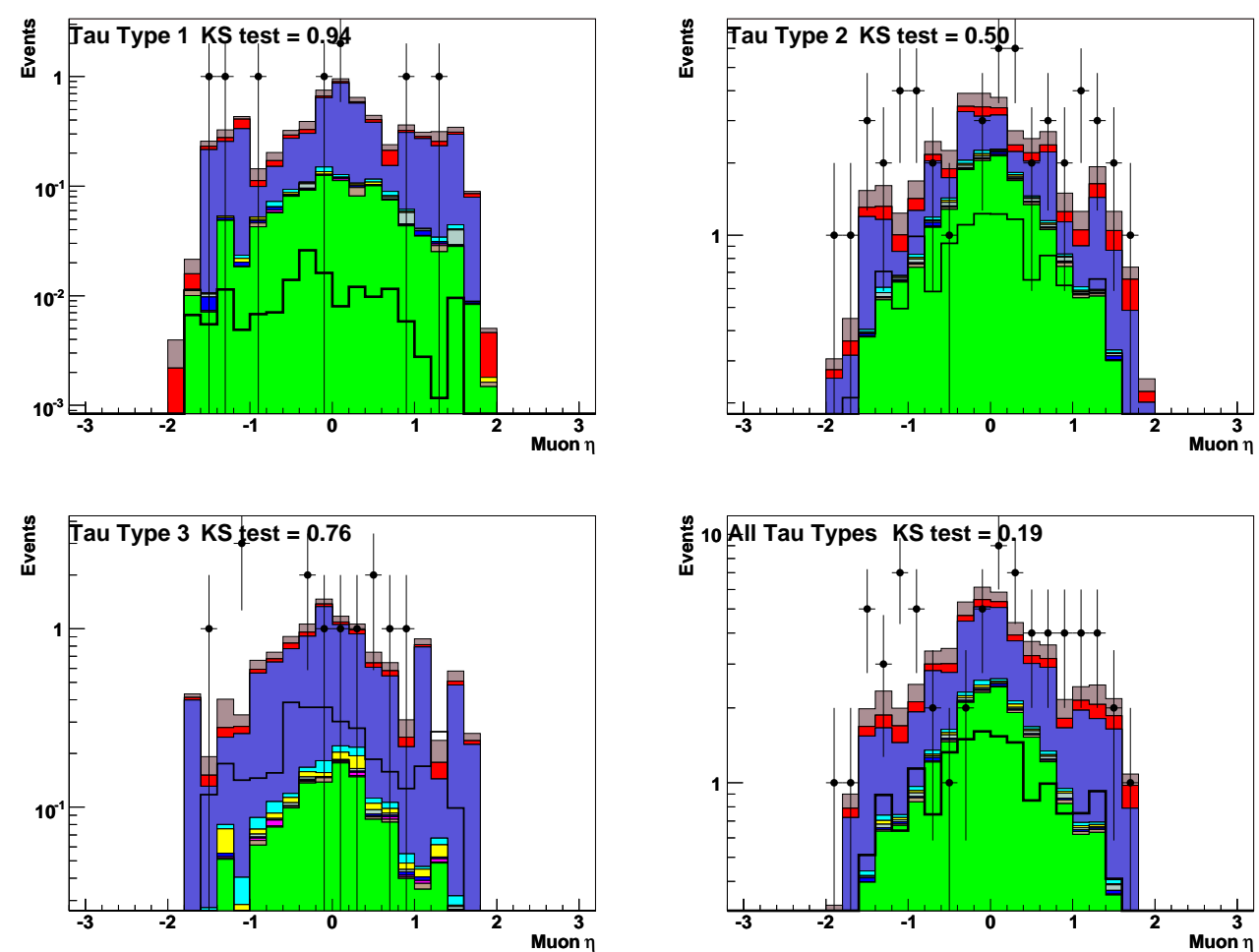

Figure B.33: Muon $\eta$ after $b$-tagging for: Type 1 taus (upper left), Type 2 (upper right), Type 3 (lower left), and all types (lower right). 

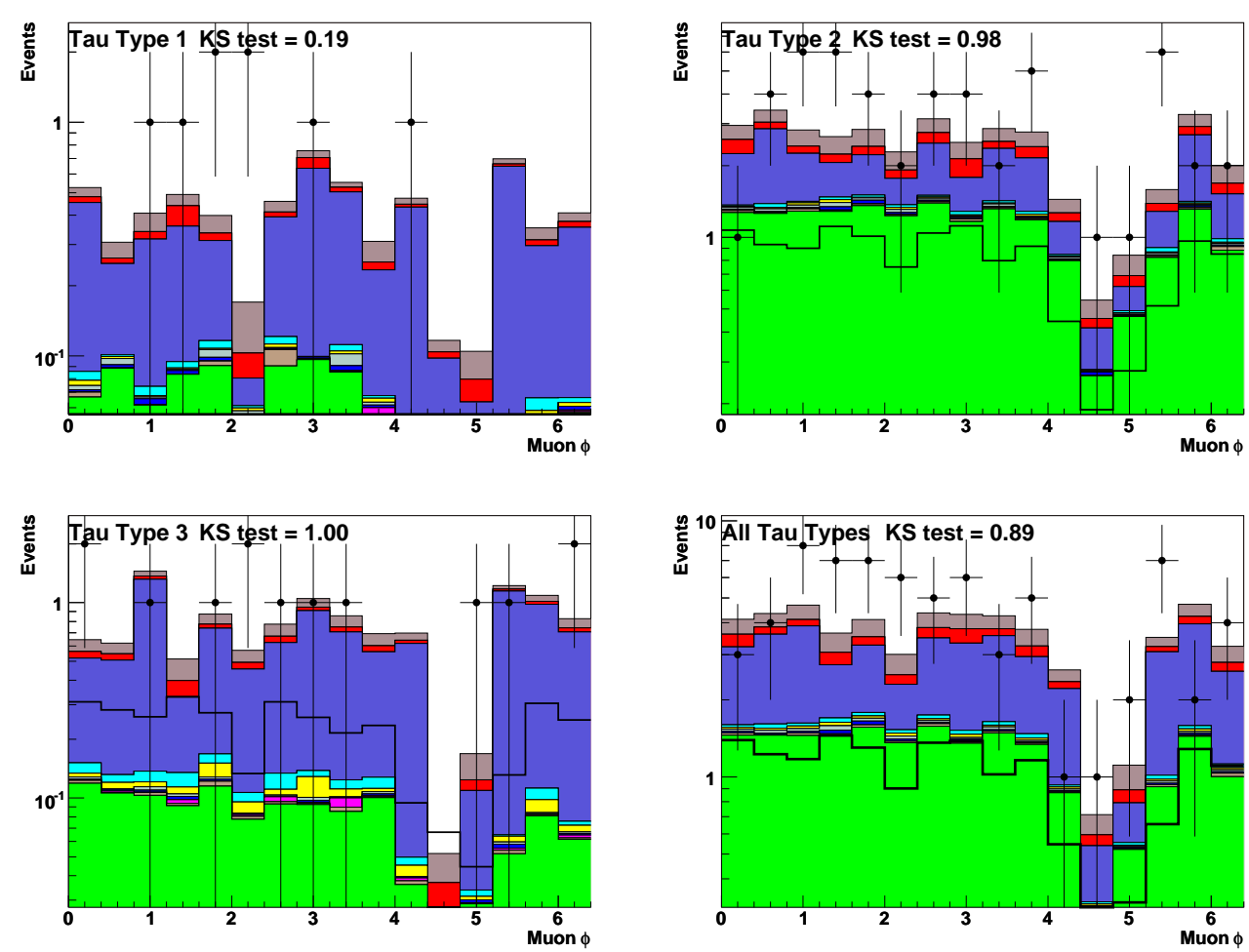

Figure B.34: Muon $\phi$ after b-tagging for: Type 1 taus (upper left), Type 2 (upper right), Type 3 (lower left), and all types (lower right). The pronounced dip around $\phi=5$ is due to the lack of full instrumentation at the bottom of the detector. 

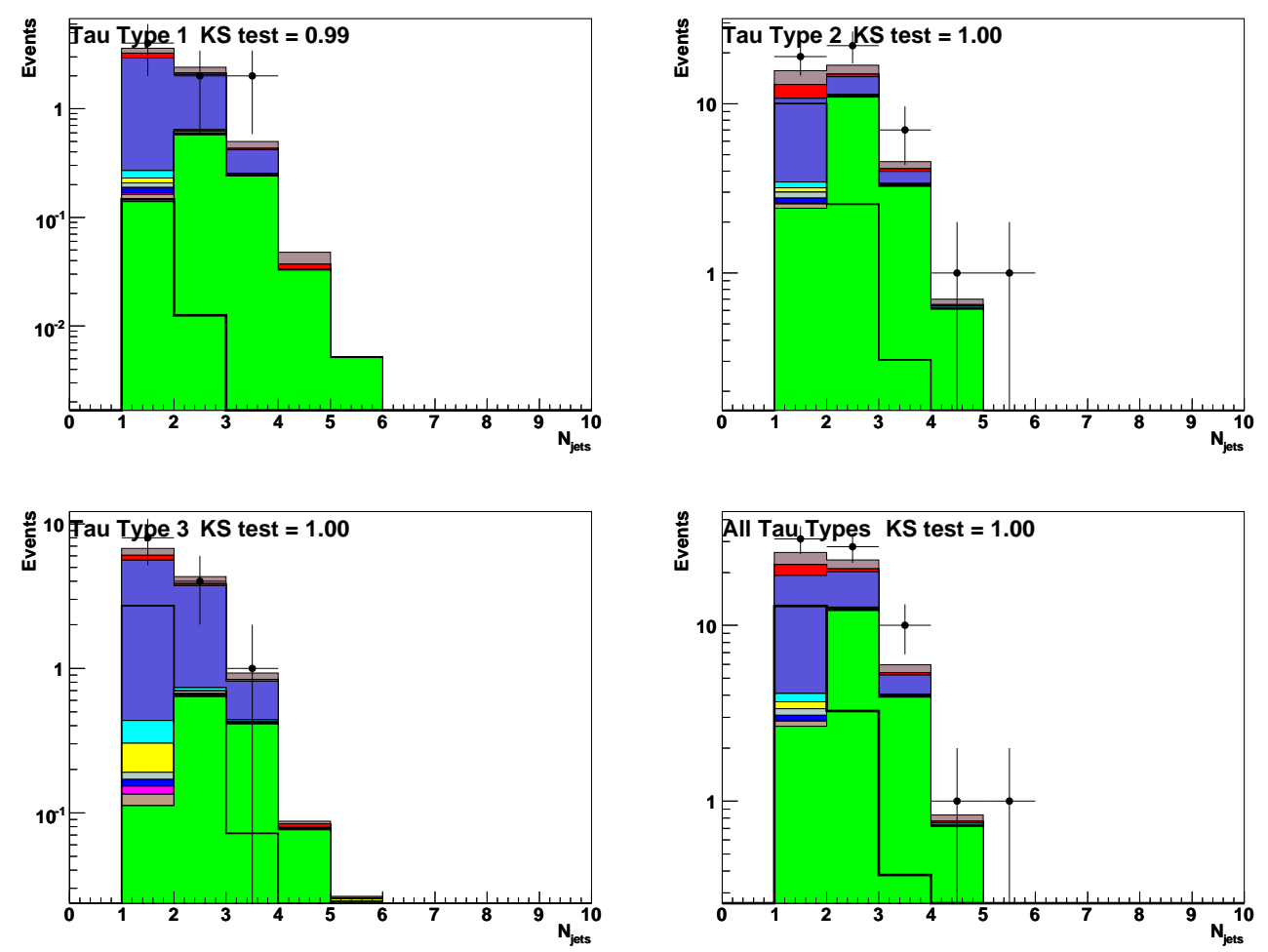

Figure B.35: Jet multiplicity after b-tagging for: Type 1 taus (upper left), Type 2 (upper right), Type 3 (lower left), and all types (lower right). 

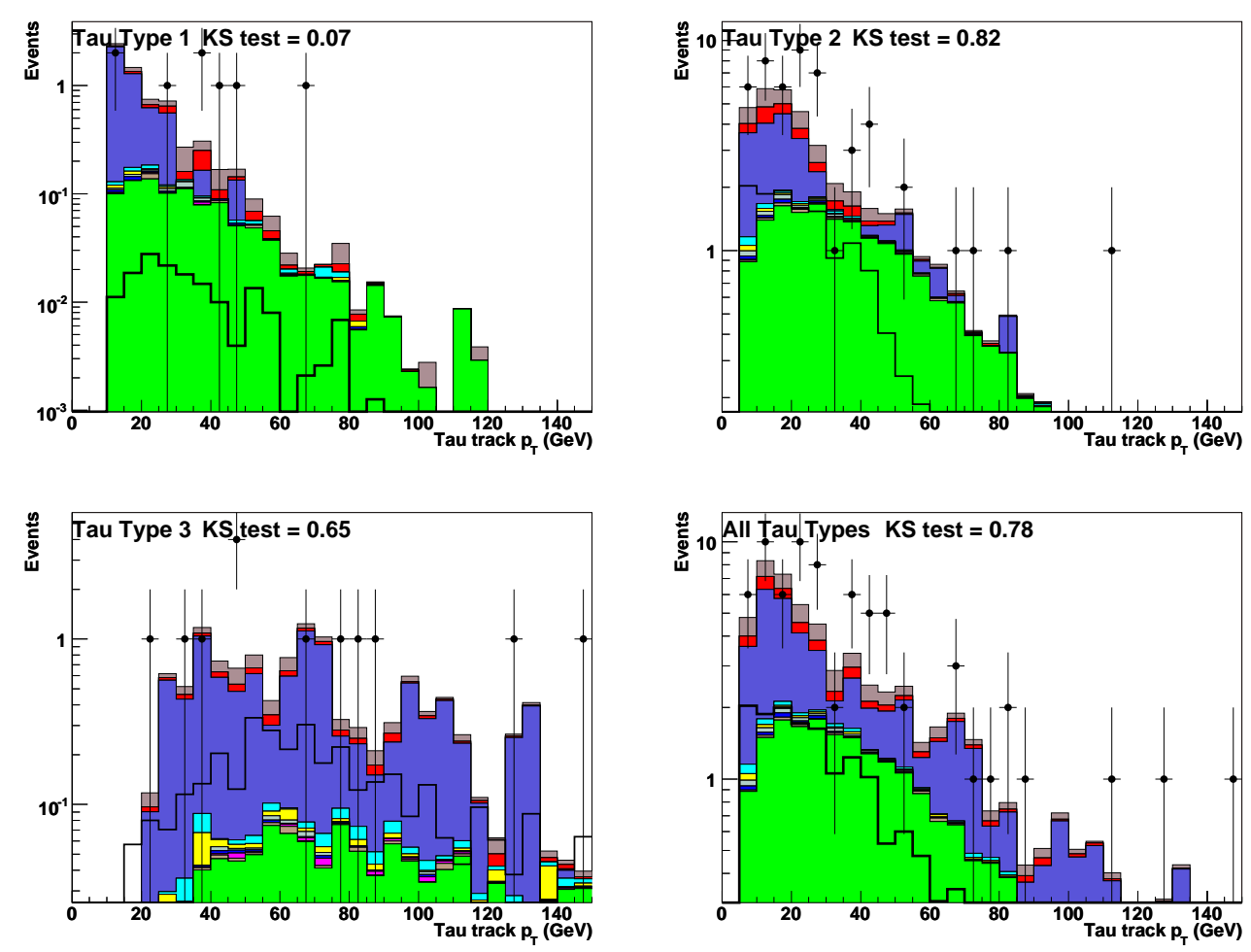

Figure B.36: Tau associated track $p_{T}$ after $b$-tagging for: Type 1 taus (upper left), Type 2 (upper right), Type 3 (lower left), and all types (lower right). In tau type 3 , it is the $p_{T}$ sum of all tracks. 

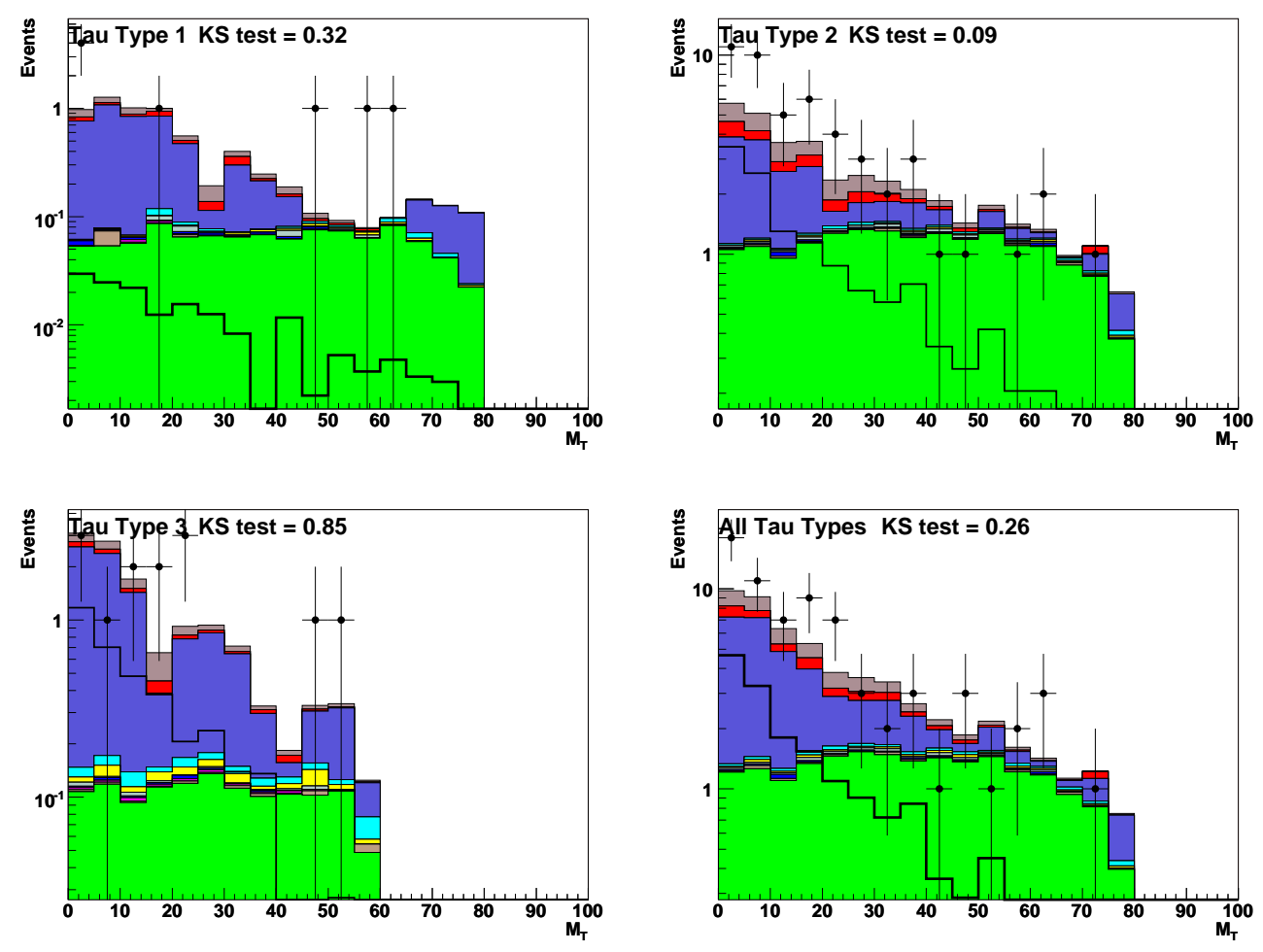

Figure B.37: The transverse mass $\left(M_{T}=\sqrt{\left(2 \mathbb{E}_{T} E_{T \mu}\left(1-\cos \left(\Delta \phi\left(\mu, \mathbb{E}_{T}\right)\right)\right)\right.}\right)$ after $b$-tagging for: Type 1 taus (upper left), Type 2 (upper right), Type 3 (lower left), and all types (lower right). 

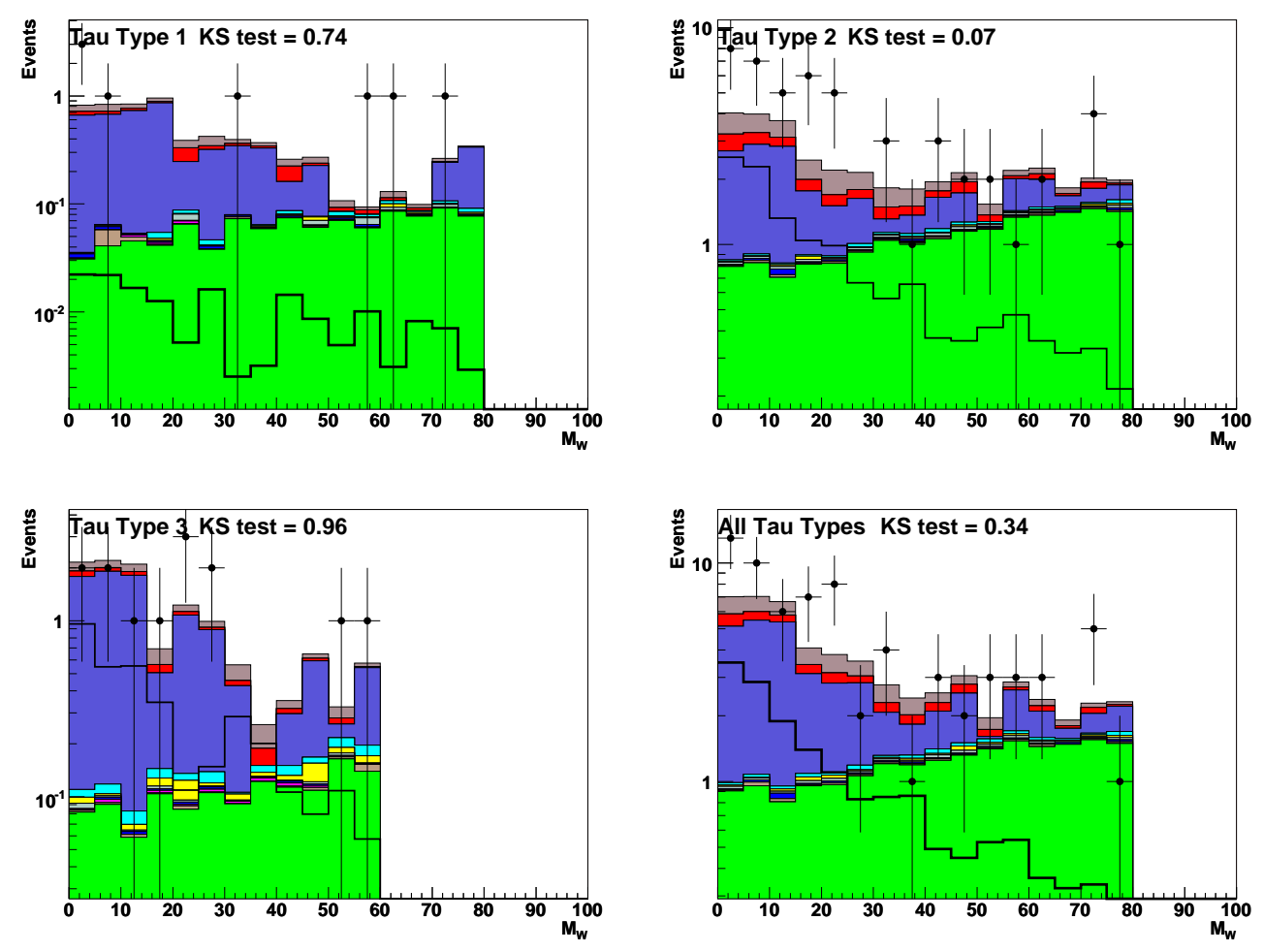

Figure B.38: The $W$ mass variable $\left(M_{W}=\sqrt{\left(2 \mathbb{E}_{T} E_{\mu}^{2} / p_{T \mu}\left(1-\cos \left(\Delta \phi\left(\mu, \mathbb{E}_{T}\right)\right)\right)\right.}\right)$ after $b$-tagging for: Type 1 taus (upper left), Type 2 (upper right), Type 3 (lower left), and all types (lower right). 

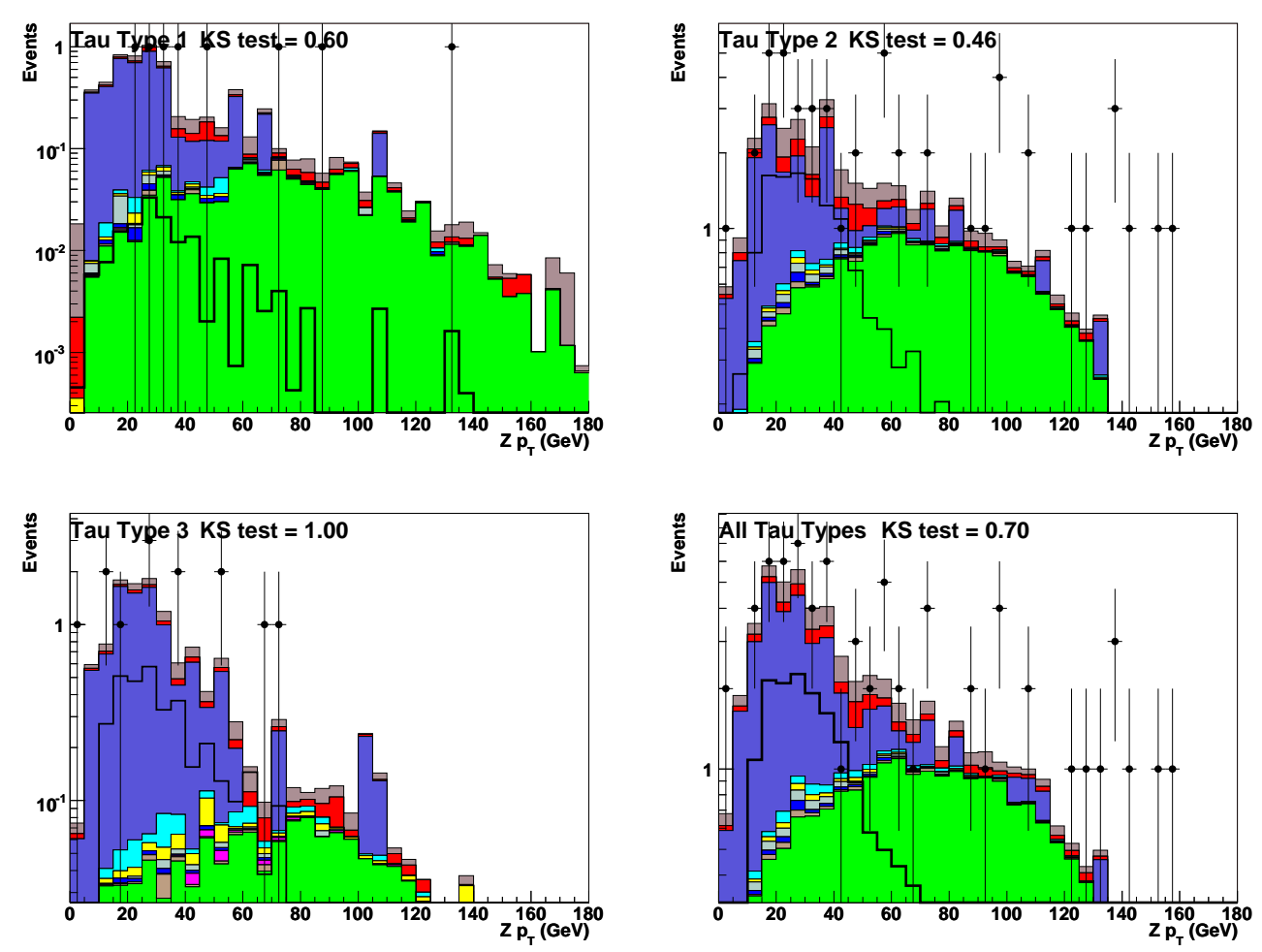

Figure B.39: The $Z p_{T}$ variable, $Z p_{T}=\left|\boldsymbol{p}_{\boldsymbol{T}}^{\boldsymbol{\mu}}+\boldsymbol{p}_{\boldsymbol{T}}^{\boldsymbol{\tau}}+\boldsymbol{E}_{\boldsymbol{T}}\right|$, after $b$-tagging for: Type 1 taus (upper left), Type 2 (upper right), Type 3 (lower left), and all types (lower right) using the Single Muon or Mu+Tau OR. 


\section{Appendix $\mathrm{C}$}

\section{QCD Likelihood Control Plots}

In this appendix we show the smoothing functions used in the QCD likelihood for each input variable and detail the functions themselves. We begin by listing the smoothing function forms used for each variable in the likelihood:

- Muon $p_{T}$ : QCD: $A e^{\left(\frac{\left(B-p_{T \mu}\right)}{C}-e^{\frac{\left(B-p_{T \mu}\right)}{C}}\right)}$ Signal: $A e^{-\frac{\left(p_{T \mu}-B\right)^{2}}{C^{2}}}$

- Tau $p_{T}$ : QCD: $A e^{-\frac{\left(p_{T \mu}-B\right)^{2}}{C^{2}}}+D e^{-\frac{\left(p_{T \mu}-E\right)^{2}}{F^{2}}}$ Signal: $A e^{-\frac{\left(p_{T \tau}-B\right)^{2}}{C^{2}}}$

- $\Delta R(\mu, \tau)$ : QCD: $A e^{-\frac{\left(p_{T \mu}-B\right)^{2}}{C^{2}}}+D e^{-\frac{\left(p_{T \mu}-E\right)^{2}}{F^{2}}}$ Signal: $A e^{-\frac{\left(p_{T \mu}-B\right)^{2}}{C^{2}}}+D e^{-\frac{\left(p_{T \mu}-E\right)^{2}}{F^{2}}}$

- $\mu, \tau$ invariant mass $\left(M_{\mu, \tau}\right)$ : QCD: $\frac{A}{(x-B)^{2}+C^{2}}$ Signal: $A e^{-\frac{\left(p_{T \tau}-B\right)^{2}}{C^{2}}}$ for $\mathrm{M} \leq$ $120 \mathrm{GeV}, A e^{-\frac{\left(p_{T \mu}-B\right)^{2}}{C^{2}}}+D e^{-\frac{\left(p_{T \mu}-E\right)^{2}}{F^{2}}}$ for $\mathrm{M}>120 \mathrm{GeV}$.

- Visible mass $M\left(\mu, \tau \mathbb{E}_{T}\right)$ : QCD: $A e^{\left(\frac{\left(B-p_{T \mu}\right)}{C}-e^{\frac{\left(B-p_{T \mu}\right)}{C}}\right)}$ Signal: $A e^{-\frac{\left(p_{T \mu}-B\right)^{2}}{C^{2}}}+$ $D e^{-\frac{\left(p_{T \mu}-E\right)^{2}}{F^{2}}}$

We fit the distributions with the results shown in the following figures. For the signal we fit each mass point separately. Figures C.1 through C.5 are the QCD results, while figures C.6 through C.10 are the signal results. 


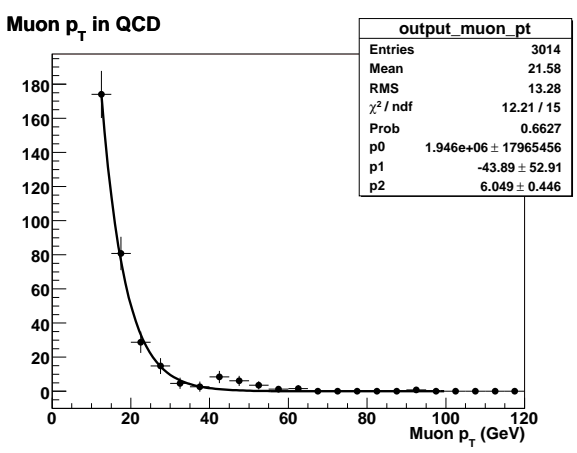

Figure C.1: Muon $p_{T}$ smoothing functions on the QCD background for the QCD likelihood.

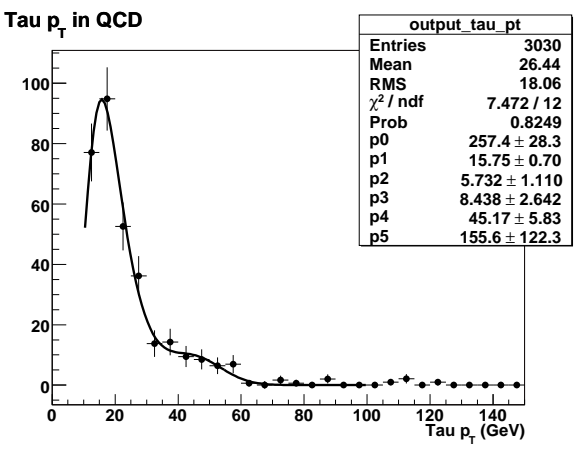

Figure C.2: Tau $p_{T}$ smoothing functions on the QCD background for the QCD likelihood.

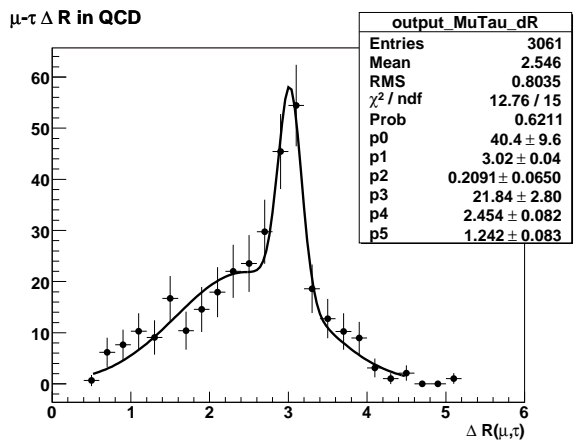

Figure C.3: $\Delta R(\mu, \tau)$ smoothing functions on the QCD background for the QCD likelihood. 


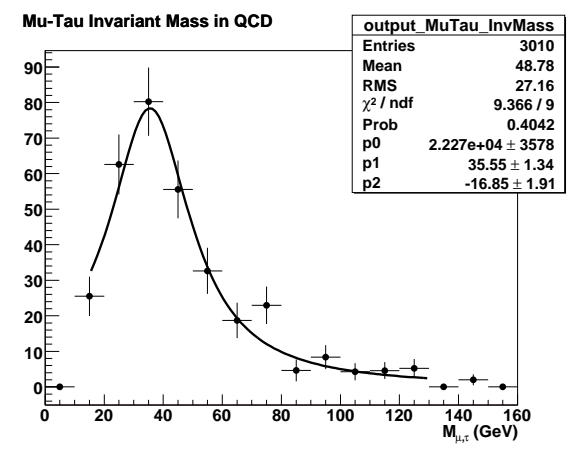

Figure C.4: $\mu, \tau$ invariant mass smoothing functions on the QCD background for the QCD likelihood.

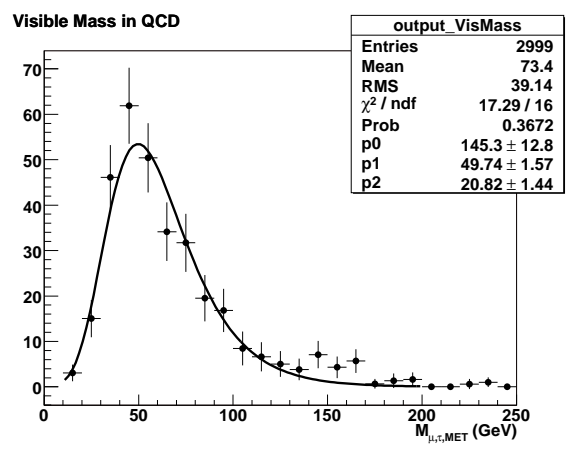

Figure C.5: $\mu, \tau \mathbb{E}_{T}$ invariant mass smoothing functions on the QCD background for the QCD likelihood. 

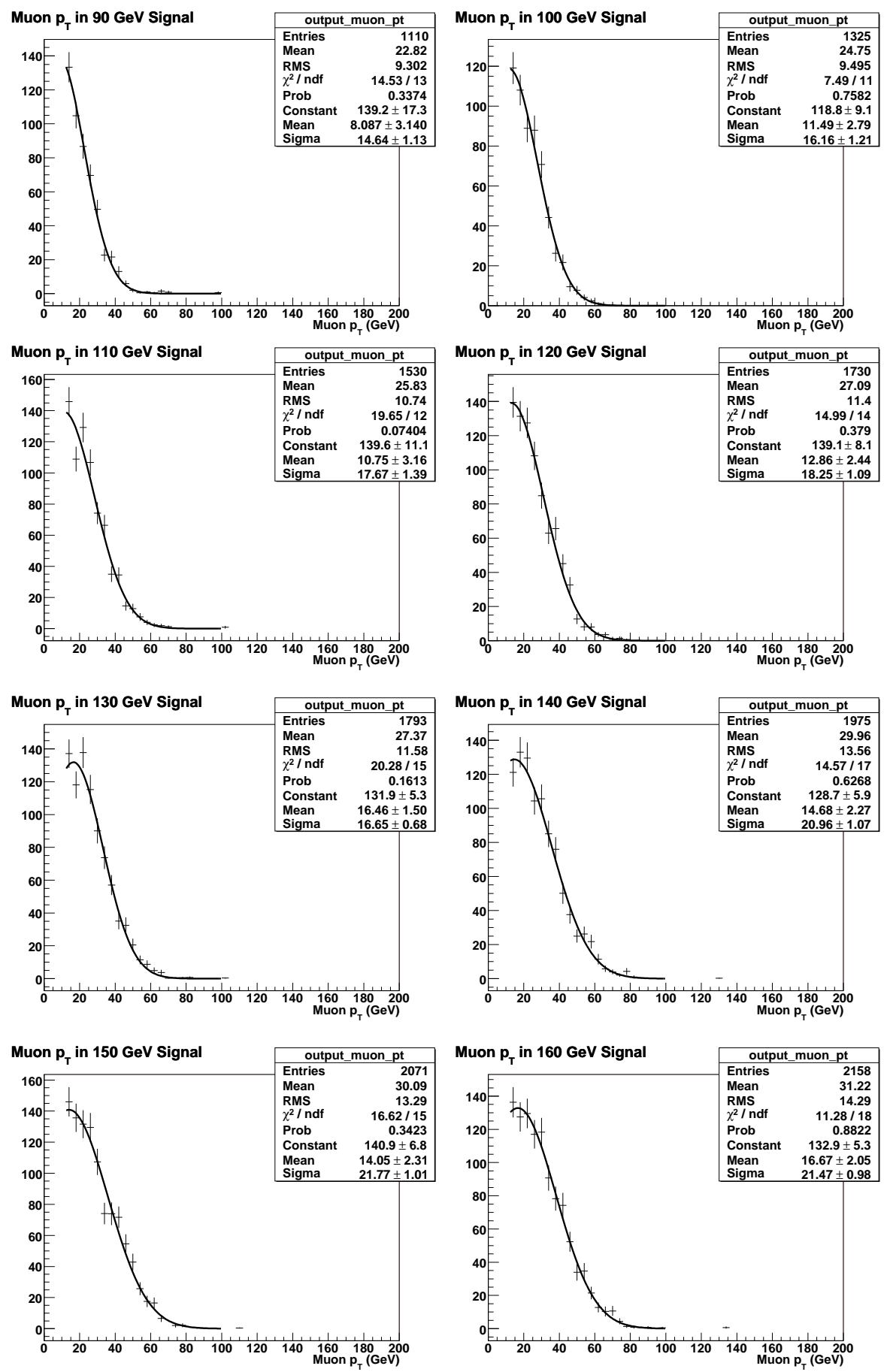

Figure C.6: Muon $p_{T}$ smoothing functions for signal likelihood for the different Higgs masses. Row 1: $90 \mathrm{GeV}$ (left), $100 \mathrm{GeV}$ (right). Row 2: $110 \mathrm{GeV}$ (left), $120 \mathrm{GeV}$ (right). Row 3: $130 \mathrm{GeV}$ (left), $140 \mathrm{GeV}$ (right). Row 4: $150 \mathrm{GeV}$ (left), $160 \mathrm{GeV}$ (right). 

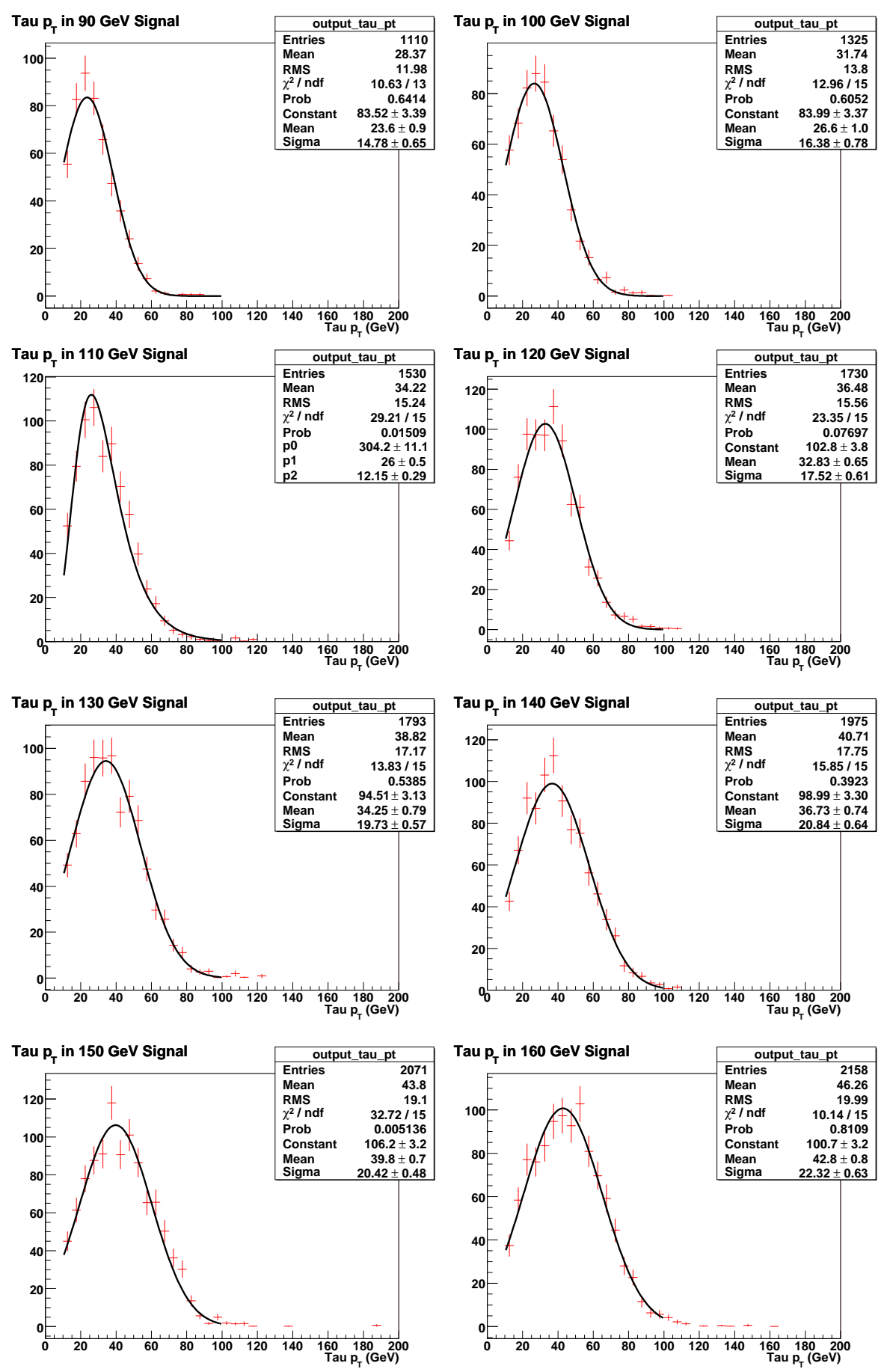

Figure C.7: Tau $p_{T}$ smoothing functions for signal likelihood for the different Higgs masses. Row 1: $90 \mathrm{GeV}$ (left), $100 \mathrm{GeV}$ (right). Row 2: $110 \mathrm{GeV}$ (left), $120 \mathrm{GeV}$ (right). Row 3: $130 \mathrm{GeV}$ (left), $140 \mathrm{GeV}$ (right). Row 4: $150 \mathrm{GeV}$ (left), $160 \mathrm{GeV}$ (right). 

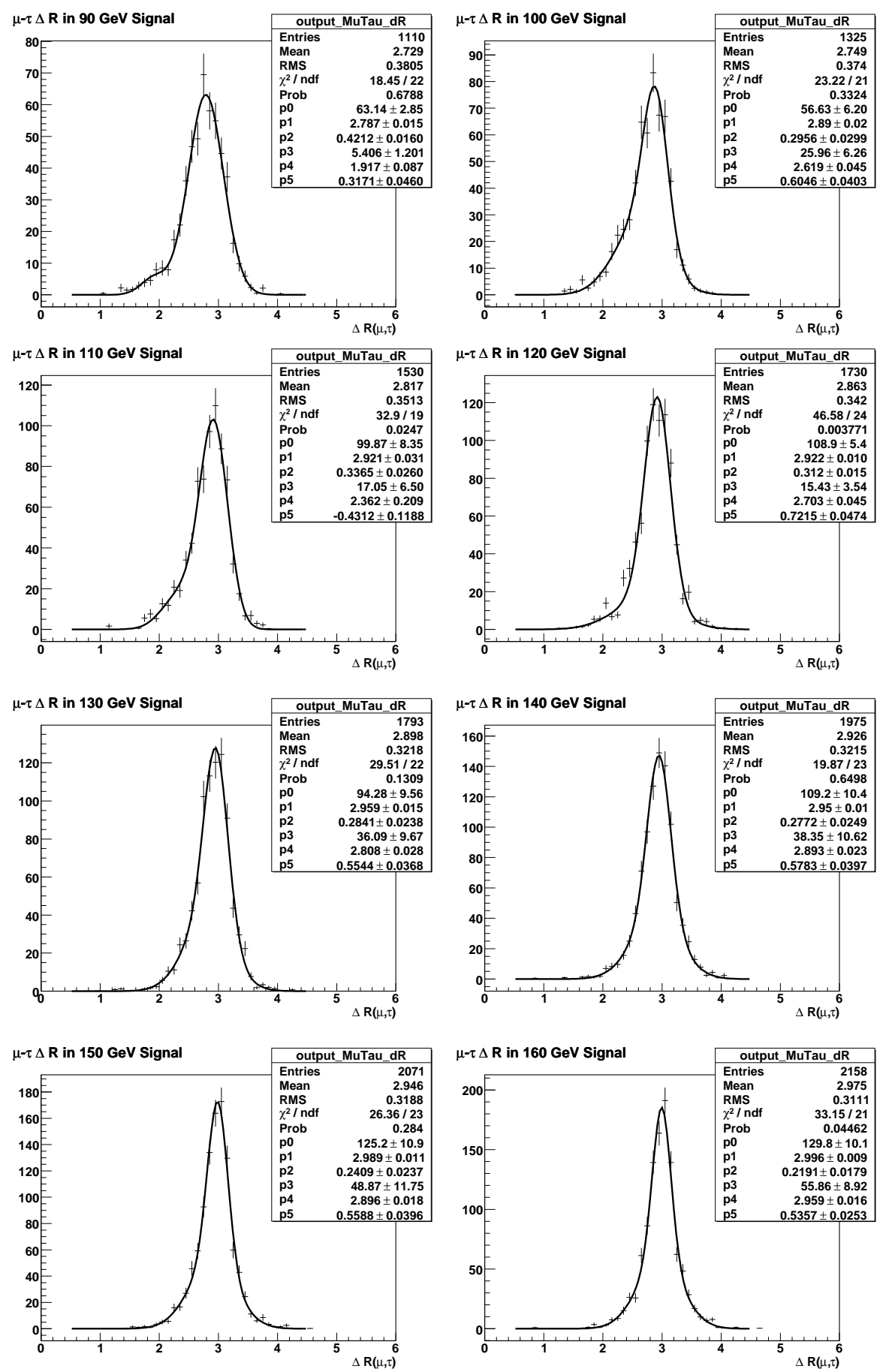

Figure C.8: $\Delta R(\mu, \tau)$ smoothing functions for signal likelihood for the different Higgs masses. Row 1: $90 \mathrm{GeV}$ (left), $100 \mathrm{GeV}$ (right). Row 2: $110 \mathrm{GeV}$ (left), $120 \mathrm{GeV}$ (right). Row 3: $130 \mathrm{GeV}$ (left), $140 \mathrm{GeV}$ (right). Row 4: $150 \mathrm{GeV}$ (left), $160 \mathrm{GeV}$ (right). 

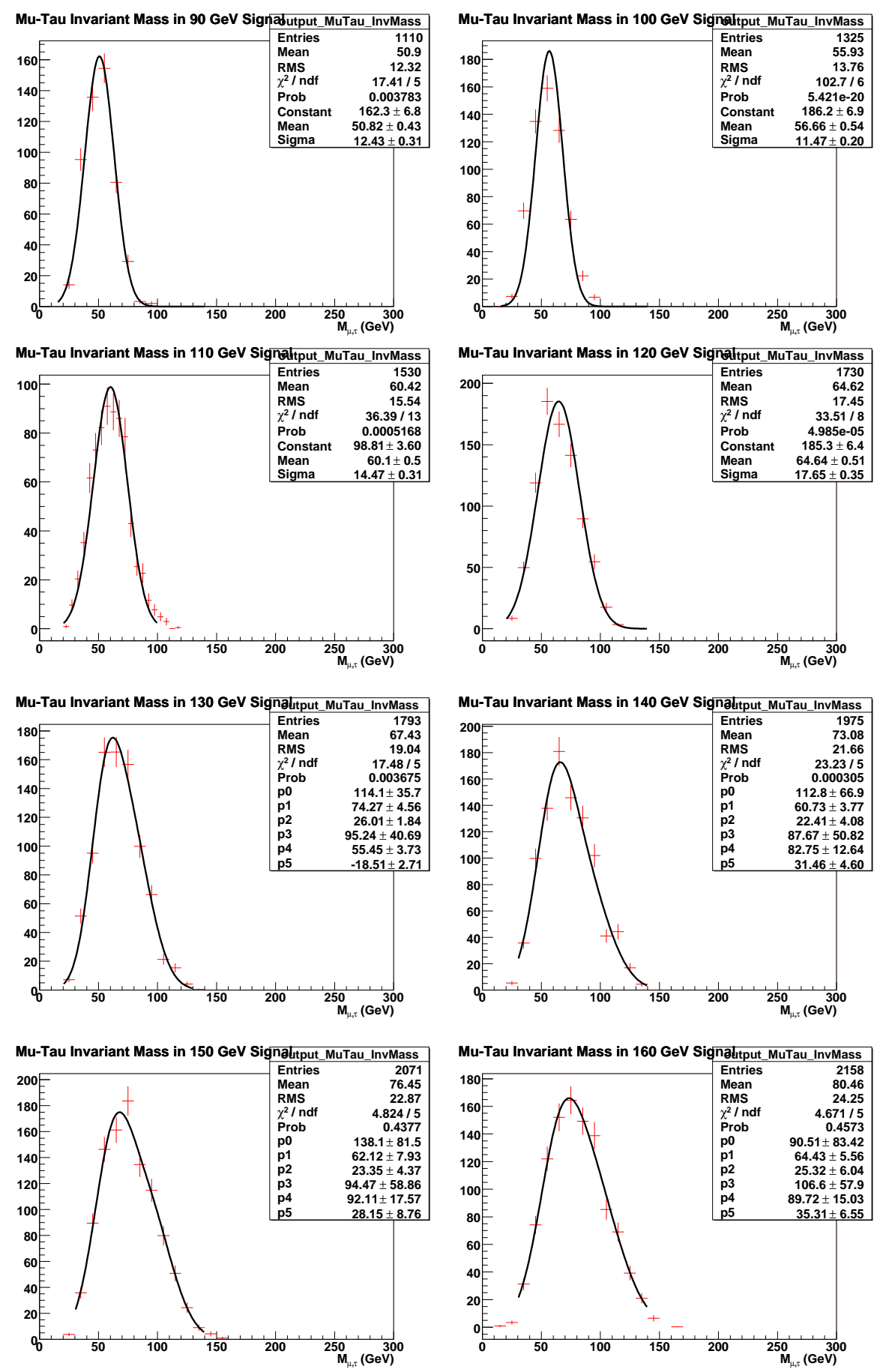

Figure C.9: $\mu, \tau$ invariant mass smoothing functions for signal likelihood for the different Higgs masses. Row 1: $90 \mathrm{GeV}$ (left), $100 \mathrm{GeV}$ (right). Row 2: $110 \mathrm{GeV}$ (left), $120 \mathrm{GeV}$ (right). Row 3: $130 \mathrm{GeV}$ (left), $140 \mathrm{GeV}$ (right). Row 4: $150 \mathrm{GeV}$ (left), $160 \mathrm{GeV}$ (right). 

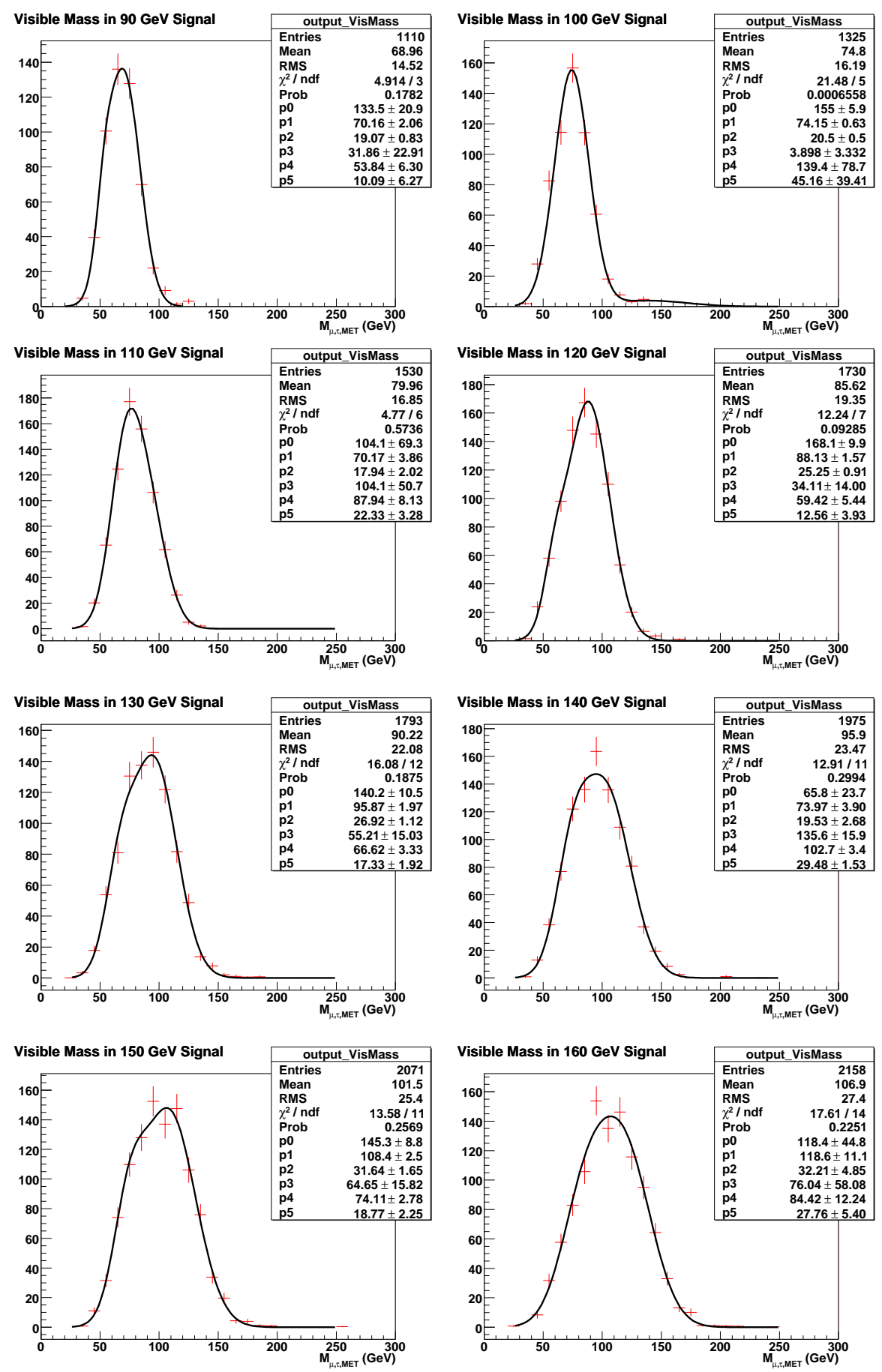

Figure C.10: $\left(\mu, \tau, \mathbb{E}_{T}\right)$ invariant mass smoothing functions for signal likelihood for the different Higgs masses. Row 1: $90 \mathrm{GeV}$ (left), $100 \mathrm{GeV}$ (right). Row 2: $110 \mathrm{GeV}$ (left), $120 \mathrm{GeV}$ (right). Row 3: $130 \mathrm{GeV}$ (left), $140 \mathrm{GeV}$ (right). Row 4: $150 \mathrm{GeV}$ (left), $160 \mathrm{GeV}$ (right). 